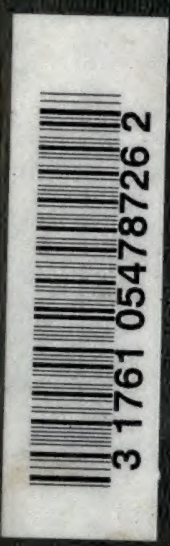




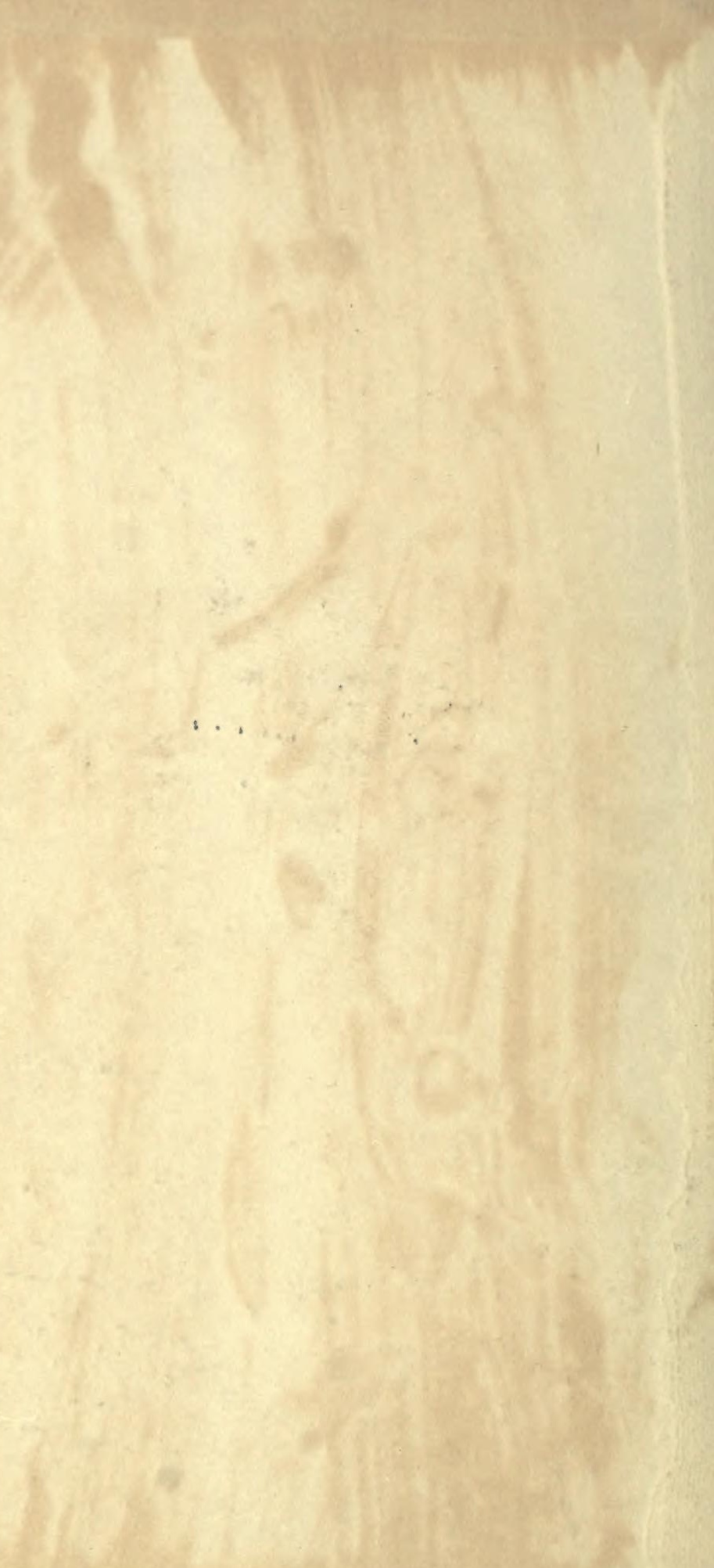




\section{LIBRARY \\ FACULTY OF FORESTRY \\ UNIVERSITY_OF TORONTO \\ NOV 5 - 1905}




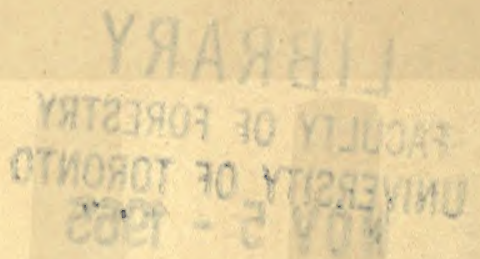

Digitized by the Internet Archive in 2007 with funding from Microsoft Corporation 


\section{CATALOGUE}

$-\mathrm{OF}-$

North American Plants

\section{North of Mexico,}

Exclusive of the lower cryptogams.

By A. A. HELLER.

$2 d$. ed

$[x, p$.

Second Edition. Issued November ro, 1900.

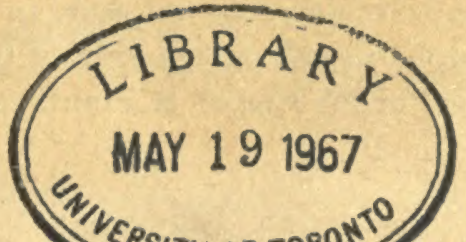

2) 
AвоU' 2000 new plant names having been published since the first edition of this Catalogue was issued in March, 1898 , the time seems ripe for a second edition. The aim of the author has been to make it as near complete and perfect as possible, and with this end in view, more labor and expense has been put upon it than is usual in a publication of this class.

The page is printed upon one side only, giving space for the insertion of new names, which are constantly being added. Larger type has been used, which will be appreciated by many, and the press work is of the best. The "Pluck Art Printery," where our press-work is done, has the reputation of putting out nothing but the finest kind of work.

The intention was to number the genera throughout according to their sequence in Engler \& Prantl's "Naturlichen Pflanzenfamilien," as set forth by Drs. Torre and Harms, in their "Genera Siphonogamarum." The first fascicle only of this latter work had appeared when the Catalogue was begun, the last available number in it being Iris I264. From that point on the calculations were made direct from Engler \& Prantl, where the number of the genus in the family is given, but not the total number in the sequence. In some way a miscount occurred, as at once became evident when fascicle 2 of the "Genera" arrived. The numbering was at once discontinued, and all numbers after Nemastylis $\mathbf{1 2 7 4}$ should be ignored, as they are not correct.

The author supposed that the universal acceptance of the change from the obsolete arrangement of Bentham \& Hooker to the more rational one of Engler \& Prantl was understood by all, but a complaint that the position of the families was reversed in the first edition, seems to call for this remark.

Instead of repeating the abbreviation var. before each varietal name, the name is set further back under the species.

The almost unanimous demand for a natural arrangement by those who answered my circular, sent out early in the year, led to the abandonment of the alphabetical arrangement of the first edition, and the adoption throughout of the Engler \& Prantl sequence for families 
and genera. Strict adherence to this sequence has resulted in the retaining of but one family name in some cases where several seem more appropriate, and more in accordance with modern views.

Many species described by Nuttall, Pursh, and other botanists who really knew the plants they described from actual acquaintance with them in the living state, have either been suppressed altogether, or recorded as varieties by botanists who knew them only as herbarium specimens. These species are constantly being reinstated by field workers of to-day, and help to swell the list of names.

There is also a growing tendency to discard the use of varietal names, and to call all plants species which have characters enough to justify the use of a distinctive name. Accordingly, many varieties have been raised to specific rank, and may be looked for in their proper place in the alphabetical arrangement of species.

Several persons have suggested that the number given before each species should not be changed, but remain the same in successive editions of this work, added species being indicated by a, b, etc. The suggestion is a good one, but until the whole country is thoroughly worked over, and numerous genera pared down to their proper limits) it does not seem possible.

At the present rate of activity in taxonomic botany, the year I 905 will see 20,000 plant names to be listed, and many changes in generic limits.

It will be esteemed a favor if errors, however trivial, are reported, as well as any plant names which do not appear in this book, and which were published prior to October, r9oo. It is suggested that errors and omissions be noted on the printed page, or in red ink, in order to easily distinguish them from the additions.

While preparing the manuscript for this edition, it became apparent that a number of new combinations would have to be published in order to secure uniformity of treatment, some of which are given below.

Agropyron Smithii Palmeri (Scribn. \& Smith)

Agropyron spicatum Palmeri Scribn. \& Smith, U. S. Dept. Ag. Div. Agrost. Bull. 4: 33. 1897 .

Agropyron spicatum inerme (Scribn. \& Smith)

Agropyron divergens inerme Scribn. \& Smith, U. S. Dept. Ag. Div. Agrost. Bull. 4: 27. I897.

Carex pratericola furva (Bailey)

Carex pratensis var. furva Bailey; Macoun, Cat. Can. P1. 2:

377. I 890 . 
Vagnera amplexicaulis brachystyla (Henderson)

Smilacina racemosa brachystyla Henderson, Bull. Torr. Club, 27: 357 . 1900 .

Cephalanthera Austinae (A. Gray)

Chloraea Austinae A. Gray, Proc. Am. Acad. 12: 83. 1876.

Cephalanthera Oregana Reichenb. f. Linnaea, 41: 53. $\quad$ I877.

Peramium repens ophioides (Fernald)

Goodyera repens var. ophioides Fernald, Rhodora, 1: 6. 1899.

Peramium tesselatum (Lodd.)

Goodyera tesselata Lodd. Bot. Cab. 10: no. $952 . \quad 1824$.

\section{Salix Rydbergii}

Salix Macounii Rydb. Bull. N. Y. Bot. Gard. 1: 269. I899; not Salix Richardsonii var. Macouniana Bebb.

Salix tenerrima (Henderson)

Salix longifolia tenerrima Henderson, Bull. Torr. Club, 27: 354. 1890.

Asarum majus (Ducharte)

Asarum Hookeri var. majus Ducharte in DC. Prod. 15: Part I, 424. I 844 .

Asarum Hartwegi Wats. Proc. Am. Acad. 10: $346 . \quad 1875$.

Alsine Jamesiana (Torr.)

Stellaria Jamesiana Torr. Ann. Lyc. N. Y. 2: 169. 1828.

Stellaria Jamesii Torr. Pac. R. R. Surv. 4: 69. 1857.

Alsine Jamesii Holzinger, Cont. U. S. Nat. Herb. 3: 2 I6. I 895.

Alsine oxyphylla (Robinson)

Stellaria oxyphylla Robinson, Bot Gaz. 25: 165. 1898.

Alsine Washingtoniana (Robinson)

Stellaria Washingtoniana Robinson, Bot. Gaz. 25: I66. 1898.

Pulsatilla Ludoviciana (Nutt.)

Anemone Ludoviciana Nutt. Gen. 2: 20. I8I8.

Anemone Nuttalliana DC. Syst. 1: I93. I8I8.

Anemone patens var. Nuttalliana A. Gray, Man. Ed. 5, 36. I867

Pulsatilla hirsutissima Britton, Ann. N. Y. Acad. Sci. 6: 217. I89I ; not Clematis hirsutissima Pursh.

Sophia sophioides (Fisch.)

Sisymbrium sophioides Fisch. in Hook. Fl. Bor. Am. 1: 61, pl. 20. 1830 .

Sisymbrium Sophia var. sophioides B. \& H. Gen. 1: $78 . \quad$ I867.

Phoenicaulis Menziesii lanuginosa (Wats.)

Parrya Menziesii var. lanuginosa Wats. Syn. Fl. 1: Part I, I52. I895. 
Therofon Purpusi ('T. S. Brandegee)

Boykinia Purpusi T. S. Brandegee, Bot Gaz. 27: 447. 1899.

\section{Ribes binominatum}

Ribes montanum Howell, F1. N. W. Am. 1: 210. I898. Name preoccupied.

Opulaster alternans (Jones)

Neillia opulifolia var. alternans Jones, Zoe, 4: 42. 1893.

Neillia alternans Jones, 1. c. 43 , as synonym.

Petrophyton elatior (Wats.)

Spiraea caespitosa var. elatior Wats. U. S. Geolog. Surv. 4oth Par. 5: 81. I 87 I.

Zygia Guadalupensis (Desv.)

Inga Guadalupensis Desv. Journ. Bot. 3: 70. I8I4.

Pithecolobium Guadalupense Chapm. Fl. Ed. 2, II6. I883.

Acuan Fendleri (Wats.)

Desmanthus Jamesii var. Fendleri Wats. Bibl. Index, 2I4. x878.

Acuan glandulosa (Michx.)

Mimosa glandulosa Michx. Fl. Bor. Am. 2: 254. I803.

Desmanthus brachylobus var. glandulosus Engelm. \& Gray, Bost. Journ. Nat. Hist. 5: 243. I845.

Chamaecrista aspera Mohrii Pollard

Cassia aspera Mohrii Pollard.

Chamaecrista cinerea (Cham. \& Schlecht.) Pollard.

Cassia cinerea Cham. \& Schlecht. Linnaea, 5: 599. 1830.

Chamaecrista Greggii (A. Gray) Pollard.

Cassia Greggii A. Gray, P1. Wright. 1: $59 . \quad$ I $85^{2}$.

Chamaecrista Mississippiensis Pollard.

Cassia Mississippiensis Pollard, Bull. Torr. Club, 21:2 I9. I894.

Chamaecrista robusta Pollard.

Cassia Chamaecrista robusta Pollard, Bull. Torr. Club, 21: 218. I894.

Chamaecrista Simpsoni Pollard.

Cassia Simpsoni Pollard, Bull, Torr. Club, 21: 221. I894.

Parosela arborescens (Torr.)

Dalea arborescens Torr.; A. Gray, Mem. Am. Acad. II. 5: 3I6. I 855 .

Parosela argyraea (A. Gray)

Dalea argyraea A. Gray, Pl. Wright, 1: 47. $\quad$ I 852.

Parosela calycosa (A. Gray)

Dalea calycosa A. Gray, P1. Wright. 2: 40. $\quad$ I853. 
Parosela Domingensis (DC.)

Dalea Domingensis DC. Prod. 2: $246 . \quad$ I825.

Parosela Emoryi (A. Gray)

Dalea Emoryi A. Gray, Mem. Am. Acad. II. 5: 3I5. 1855.

Parosela fliformis (A. Gray)

Dalea filiformis A. Gray, P1. Wright. 2: 39. 1853.

Parosela Greggii (A. Gray)

Dalea Greggii A. Gray, Mem. Am. Acad. II. 5: 3I4. 1855 .

Parosela Kingii (Wats.)

Dalea Kingii Wats. U. S. Geolog. Surv. 4oth Par. 5: 64. I87r.

Parosela lachmostachys (A. Gray)

Dalea lachnostachys A. Gray, P1. Wright. 1: $46 . \quad$ I 852.

Parosela lasiathera (A. Gray)

Dalea lasiathera A. Gray, Pl. Wright. 1: 48. I 852.

Parosela Lemmoni (Parry)

Dalea Lemmoni Parry, in Gray, Proc. Am. Acad. 1\%: 200.1882.

Parosela mollis (Benth.)

Dalea mollis Benth. P1. Hartw. 306. I 848 .

Parosela Neo-Mexicana (A. Gray)

Dalea mollis var.? Neo-Mexicana A. Gray, Pl. Wright. 1: 47.

Parosela Ordiae (A. Gray)

Dalea Ordiae A. Gray, Proc. Am. Acad. 1\%: 200. I882.

Parosela Parryi ('T. \& G.)

Dalea Parryi T. \& G. Proc. Am. Acad. \%: 297. I 868.

Parosela polydenia (Torr.)

Dalea polydenia Torr.; Wats. U. S. Geolog. Surv. 4oth Par. 5:

64. 1871 .

Parosela polygonoides (A. Gray)

Dalea polygonoides A. Gray, P1. Wright. 2: $39 . \quad 1853$.

Parosela Pringlei (A. Gray)

Dalea Pringlei A. Gray, Proc. Am. Acad. 1\%: 201. 1882.

Parosela pulchella (Moric.)

Dalea pulchella Moric. Pl. Nouv. Am. 9, pl. 7. 1833.

Parosela ramosissima (Benth.)

Dalea ramosissima Benth. Bot. Voy. Sulph. I I, pl. Io. $\quad$ I844.

Parosela scariosa (Wats.)

Dalea scariosa Wats. Proc. Am. Acad. 1\%: $369 . \quad$ I882.

Parosela Schottii (Torr.)

Dalea Schottii Torr. Bot. Mex. Bound. 53. I859. 
Parosela scoparia (A. Gray)

Dalea scoparia A. Gray, Mem. Am. Acad. II. 4: 32. I849.

Parosela spinosa (A. Gray)

Dalea spinosa. A. Gray, Mem. Am. Acad. II. 5: 3I 5. 1855.

Astragalus Malheurensis

Astragalus Cusickii Rydb. Bull. Torr. Club, 26: 54I. ז899; not A. Gray, I878.

Homalobus spatiosus (Sheldon)

Astragalus glabriusculus var. spatiosus Sheldon, Minn. Bot. Stud. 1: I56. I 894 .

Astragalus glabriusculus var. major A. Gray, Proc. Acad. Phila.

II. \%: 70. I 863 ; not $A$. subulatus var. major DC., 1835 .

Aragallus campestris caeruleus (Koch)

Oxytropis campestris var. caerulea Koch, Synop. I8I.

Aragallus Johannensis (Fernald)

Oxytropis campestris var. Johannensis Fernald, Rhodora, 1: 88. I 899 .

Cracca gracillima (Robinson)

Tephrosia ambigua var. gracillima Robinson, Bot. Gaz. 28: 20 I. I899.

Cracca flexuosa (Chapm.)

Tephrosia flexuosa Chapm.; T. \& G. Fl. N. A. 1: 297.1838.

Cracca Rugelii (Shuttlew.)

Tephrosia Rugelii Shuttlew. ; Robinson, Bot. Gaz. 28: 197. 1899.

Lotus diffusus (A. Gray)

Hosackia glabra var. diffusus A. Gray, Proc. Phila. Acad. 1863: 346. I863.

Lotus Fremonti (A. Gray)

Hosackia argophylla var. Fremonti A. Gray, Proc. Phila. Acad.

1863: 349. I 863 .

Lotus venustus (Eastwood)

Hosackia venusta Eastwood, Proc. Cal. Acad. III. 1: Io3. I898.

Geranium Parryi (Engelm.)

Geranium Fremontii var. Parryi Engelm. Am. Journ. Sci. II. 33: 405. 1862 .

Forsellesia arida (Jones)

Glossopetalon spinescens var. aridum Jones, Cont. West. Bot. 8:

- 28. 1898.

Forsellesia Nevadensis (A. Gray)

Glossopetalon Nevadense A. Gray, Proc. Am. Acad. 11:73. 1876. 
Forsellesia meionandra (Koehne)

(ilossopetalon meionandrum Koehne, Gartenflora, 43:237. I894.

Forsellesia pungens (T. S. Brandegree)

(ilussopetalon pungens T. S. Brandegee, Bot. Gaz. '27: 445 . 1899.

E(hinocereus caespitosus castaneus (Engelm.)

Cereus cacspitosus var. castaneus Engelm. Bost. Journ. Nat. Hist. 6: 203. 1850.

\section{Echinocereus viridiflorus tubulosus (Coult.)}

Cercus viridiflorus tubulosus Coult. Cont. U. S. Nat. Herb. 3: 383. 1896.

Cercus viridiftorus var. cylindricus Engelm. Proc. Am. Acad. 3: 278. I856; not Cereus cylindricus Haw.

\section{Opuntia humifusa microsperma (Engelm.)}

Opuntia Rafinesquii var. microsperma Engelm. Proc. Am. Acad. 3: 295. $\quad$ I856.

Opuntia mesacantha microsperma (Coult.) Cont. U. S. Nat. Herb. 3: $429 . \quad$ r896.

Opuntia humifusa parva (Coult.)

Opuntia mesacantha parva Coult. Cont. U. S. Nat. Herb. 3: 429. I 896.

Opuntia Rafinesquii var. minor Engelm. Pac. R. R. Rep. 4:55. 1856; not O. minor C. Muell.

Opuntia humifusa Vaseyi (Coult.)

Opuntia mesacantha Vaseyi Coult. Cont. U. S. Nat. Herb. 3: 431. I 896 .

Anogra gypsophila (Eastwood)

Oenothera albicaulis gypsophila Eastwood, Proc. Cal. Acad. III. 1: 73. 1897 .

Galpinsia Fendleri (A. Gray)

Oenothera Fendleri A. Gray, Mem. Am. Acad. II. 4: 45. I 849.

(ialpinsia filifolia (Eastwood) Wooton, in herb.

Oenothera tubicula filifolia Eastwood, Proc. Cal. Acad. III. 1: 72. 1897 .

Kneiffia Pilosella (Raf.)

Oenothera Pilosella Raf. Ann. Nat. 15. I820.

Oenothera fruticosa var. hirsuta T. \& G. F1. N. Am. 1:496. 1840.

Oenothera fruticosa var. Pilosella Small \& Heller, Mem. Torr. Club, 3: 26. I 892 .

Knciffia fruticosa Pilosella Britton, Mem. Torr. Club, 5: 234. I894. 


\section{Catalogue.}

OPHIOGLOSSACEAE

\section{Botrychium}

I biternatum (Lam.) Underw.

2 boreale (Fries) Milde

3 Coulteri Underw.

4 dissectum Spreng.

5 lanceolatum.(S.G.Gmel.) Angst.

6 Lunaria (L.) Sw.

7 matricariae (Schrank) Spreng.

8 neglectum Wood

(B. matricariaefolium A. Br.)

9 obliquum Muhl.

Io intermedium (D. C. Eaton)

I I occidentale Underw.

Underw.

I2 pumicola Coville

I3 silaifolium Pres1

I4 simplex E. Hitchcock

I 5 tenebrosum A. A. Eaton

I6 Virginianum (L。) Sw.

[The Asiatic B.ternatum, founded upon a specimen from Japan, is omitted, as it does not occur in America.]

\section{Ophioglossum}

I7 Alaskanum E. G. Britton

I8 arenarium E. G. Britton

I9 Californicum Prantl

20 crotalophoroides Walt.

2 I Engelmanni Prantl

22 pusillum Nutt.

23 vulgatum $\mathrm{L}$.
Cheiroglossa

24 palmata (I.) Presl

(Ophioglossum palmatum L.)

OSMUNDACEAE

\section{Osmunda}

25 cinnamomea L.

26 Claytoniana $\mathrm{L}$.

27 regalis $\mathrm{L}$.

\section{HYMENOPHYLLACEAE}

\section{Trichomanes}

28 Petersii A. Gray

29 radicans $\mathrm{Sw}$.

SCHIZAEACEAE

\section{Ormithopteris}

(Aneimia)

30 adiantifolia (L.) Bernh.

3 I Mexicana (Klotzsch.) Underw.

\section{Lygodium}

32 palmatum (Bernll.) Sw.

Schizaea

33 pusilla Pursh

\section{POL YPODIACEAE}

Woodsia

34 alpina (Bolton) S. F. Gray

35 glabella R. Br.

36 Ilvensis (L.) R. Br.

37 Mexicana Fee

$3^{8}$ obtusa (Spreng.) Torr.

39 Oregana D. C. Eaton

40 scopulina D. C. Faton 



\section{Filix}

\section{(Cystopteris)}

4I bulbifera (L.) Underw.

42 fragilis ( $\mathrm{L}_{4}$ ) Underw.

43 montana (Lam.) Underw.

\section{Matteuccia}

(Onoclea in part)

44 Struthiopteris (L.) Todaro (Onoclea Struthiopteris (I.) Hoffm.)

\section{Onoclea}

45 sensibilis L.

\section{Dryopteris}

(Aspidium in part)

46 Boottii (Tuckerman) Underw.

47 contermina strigosa (Fee)

Underw.

48 cristata (L.) A. Gray

49 Clintoniana (D. C. Eaton)

50 Filix-mas (L.) Schott

5 I Floridana (Hook.) Kuntze.

52 fragrans (L.) Schott

53 Goldieana (Hook.) A. Gray

54 celsa Palmer

55 marginalis (L.) A. Gray

56 montana (Vogel) Kuntze

57 Nevadensis (D. C. Faton)

Underw.

$5^{8}$ Noveboracensis (L.) A. Gray

59 patens (Sw.) Kuntze

60 patula (Sw.) Underw.

6 I rigida arguta (Kaulf.) Underw.

62 simulata Davenport

63 spinulosa (L.) Kuntze

64 dilatata (Hoffm.) Underw.

65 intermedia (Muhl.) Underw,

66 Thelypteris (L.) A. Gray

67 unita glabra (Mett.) Underw.

\section{Phegopteris}

68 alpestris (Hoppe) Mett.

69 Dryopteris (I..) Fee

7o hexagonoptera (Michx.) Fee

7 I Phegopteris (L.) Underw.

(P. polypodioides Fee)
72 reptans (Sw.) D. C. Eaton

73 Robertiana (Hoffm.) Underw.

74 tetragona (Sw.) Fee

\section{Tectaria}

(Aspidium in part)

75 trifoliata (L.) Cav.

\section{Polystichum}

(Aspidium in part)

76 acrostichoides (Michx.) Schott

77 actuleatum (L.) Roth

78 Braunii (Spenner) Lawson

79 Californicum (D. C. Eaton)

80 Lemmoni Underw.

Underw.

8I Lonchitis (L.) Roth

82 munitum (Kaulf.) Underw.

83 imbricans (D. C. Eaton)

84 inciso-serratum

Maxon

(D. C. Eaton) Underw.

85 scopulinum (D. C. Eaton)

\section{Phanerophlebia}

Maxon

(Aspidium in part)

86 auriculata Underw.

(Aspidium juglandifolium H. B. K.)

\section{Nephrolepis}

87 actita (Sw.) Presl

88 exaltata (I.) Schott

\section{Dennstaedtia}

89 punctilobula (Michx.) Moore (Dicksonia pilosiuscula Willd.)

90 cristata Maxon

[The genus Dicksonia was established upon a South American tree fern, to which our plant is not at all referable. ]

\section{Phyllitis}

(Scolopendrium)

9I Scolopendrium (L.) Newman

(Scolopendrium vulgare Smith)

\section{Camptosorus}

92 rhizophyllus (L.) Link 



\section{Asplenim}

93 acrosticlioides Sw, (A. thelypteroides Michx.)

94 angustifolium Michx.

95 Bradleyi D. C. Eaton

96 cicutarium Sw.

97 cyclosorum Rupr.

98 dentatum L.

99 ebenoides R. R. Scott

Ioo Filix-foemina (L.) Bernh.

IOI fontanum (L.) Bernh.

IO2 firmum Kunze

IO3 Glenniei Baker

IO4 monanthemum L.

ro5 montanum Willd.

Io6 myriophyllum Mett.

Io' parvulum Mart. \& Gal.

Io8 pinnatifidum Nutt.

Io9 platyneuron (L.) Oakes (A. ebeneum Ait.)

I IO Ruta-muraria L.

I I I septentrionale (L. Hoffm.

I 2 serratum L.

I 3 Trichomanes $\mathrm{L}$.

II 4 vespertinum Maxon

I 5 viride Huds.

\section{Blechnum}

II6 serrulatum Rich.

\section{Struthiopteris}

(Lomaria)

II 7 spicant (L.) Scop.

\section{Woodwardia}

Ir 8 areolata (L.) Moore ( $W$. angustifolia Smith)

II9 radicans (L.) Sm.

120 Virginica (I, ) Sm.

\section{Gymnopteris}

(Gymnogramma)

I2I hispida (Mett.) Underw.

(Gymnogramma Ehrenbergiana) I 22 triangularis (Kaulf.) Uंnderw.

\section{Pellaea}

I 23 andromedaefolia (Kaulf.) Fee I24 aspera (Hook.) Baker
I 25 atropurpurea (I.) L,ink

126 brachyptera (Moore) Baker

I 27 Breweri D. C. Eaton

I 28 Bridgesii Hook.

I 29 densa (Brack.) Hook.

I 30 flexuosa (Kaulf.) I,ink

I3I intermedia Mett.

I 32 marginata (Hook.) Baker

r 33 occidentalis (E. Nelson) Rydb.

I34 ornithopus Hook.

I 35 pulchella (Mart. \& Gal.) Fee.

I 36 ternifolia (Cav.) I,ink

I37 Wrightiana Hook.

\section{Notholaena}

I 38 Aschenborniana Klotzsch.

I 39 candida (Mart. \& Gal.) Hook.

I 40 cretacea Liebm.

I4I dealbata Pursh

I 42 Fendleri Kunze

I 43 ferruginea (Desv.) Hook.

I 44 Grayi Davenport

I 45 Hookeri D. C. Eaton

I46 I,emmoni D. C. Eaton

I 47 Newberryi D. C. Faton

I 48 nivea Desv.

I49 Parryi D. C. Eaton

I 50 Schaffneri (Fourn.) Underw.

I 5 I sinuata (Sw.) Kaulf.

I 52 tenera Gillies

\section{Cheilanthes}

I53 Alabamensis Kunze

I 54 argentea (Gmel.) Kunze

I 55 Californica (Nutt.) Mett.

I56 Clevelandi D. C. Faton

I 57 Cooperae D. C. Faton

I 58 Fatoni Davenport

I 59 Feei Moore

(C. lanuginosa, also C. gracilis)

I6o Fendleri Hook.

I6I fibrillosa Davenport

I62 gracillima D. C. Faton

I63 lanosa (Michx.) Watt

$$
\text { (C. vestita Sw.) }
$$

r64 lendigera (Cav.) Sw.

I65 lencopoda I,ink

I66 Lindheimeri Hook.

167 microphylla Sw. 

I68 myriophylla Desv.

I69 Parishii Davenport

I70 Pringlei Davenport

I I I tomentosa Link

I72 viscida Davenport

I73 Wrightii Hook.

\section{Cryptogramma}

I74 acrostichoides $\mathrm{R}$. Br.

I75 Stelleri (S. G. Gmel.) Prantl

(Pellaea gracilis, also $P$. Stelleri)

\section{Adiantum}

I76 Capillus-Veneris L.

I77 Jordani Muell.

(A. emarginatum Hook.)

I 8 pedatum L.

I79 rangiferinum Burgess

I8o tenerum Sw.

I8I tricholepis Fee

\section{Pteris}

r82 Cretica I.

I83 longifolia L.

I84 serrulata L. f.

\section{Pteridium}

(Pteris in part)

I85 aquilinum (L.) Kuhn

r86 caudatum (I.) Kuhn

I87 pseudocaudatum Clute

I88 pubescens Underw.

(Pteris aquilina var. lanuginosa)

Vittaria

I89 lineata (L.) Sw.

\section{Cheilogramma}

(Taenitis)

Igo lanceolata (L.) Blume

\section{Polypodium}

I9I Californicum Kaulf.

I92 falcatum Kellogg

I93 pectinatum L.

I94 plumula H. B. K.

I95 polypodioides $\left(\mathrm{I}_{\text {4. }}\right)$ A. S. Hitchc. (P. incanum Sw.)

196 Scouleri Hook. \& Grev.

I97 thysanolepis A. Br.
I98 vulgare $I_{\text {. }}$.

\section{Phlebodium}

(Polypodium in part)

I99 aureum (L.) R. Br.

\section{Campyloneuron}

(Polypodium in part)

200 Phyllitidis (L.) Presl

\section{Phymatodes}

(Polypodium in part)

201 Swartzii (Baker) Underw.

\section{Acrostichum}

202 aureum L.

203 lomarioides Jenman

\section{CERATOPTERIDACEAE}

\section{Ceratopteris}

204 thalictroides (L.) Brongn.

\section{MA RSILEACEAE}

\section{Marsilea}

205 macropoda Engelm.

206 quadrifolia L.

207 tenuifolia Engelm.

208 uncinata A. Br.

209 vestita Hook. \& Grev.

\section{Pilularia}

2 Io Americana A. Br.

\section{SALVINIACEAE}

\section{Azolla}

2I I Caroliniana Willd.

2 I 2 filiculoides Lam.

2 I 3 Sonomensis Greene

Salvinia

2 I4 natans (L.) Hoffm.

\section{EQUISETACEAE}

\section{Equisetum}

215 arvense $I$.

2 I6 alpestre Wahl.

217 arcticum Rupr.

2 I 8 boreale Bong.

2 I9 campestre Schultz 

220 decumbens Meyer

22 I irriguum Milde

222 nemorosum $\mathrm{A} . \mathrm{Br}$.

223 polystachyon A. A. Eaton

224 pseudo-sylvaticum Milde

225 fluviatile L.

(E. limosum L.)

226 hyemale $\mathrm{L}$.

227 laevigatum A. Br.

228 littorale Kuehl.

229 Mexicanum Milde

230 palustre $\mathrm{L}_{4}$.

23 I pratense Ehrh.

232 ramosissimum Desf.

233 robustum A. Br.

234 scirpoides Michx.

235 sylvaticum L.

236 Telmateia Ehrh.

237 variegatum Schleich.

\section{LYCOPODIACEAE}

\section{Lycopodium}

238 adpressum (Chapm.) Lloyd \& Underw.

239 alopecuroides I.

240 alpinum I.

$24 \mathrm{I}$ antotinum $\mathrm{I}$.

242 pungens Spring.

243 Carolinianum L.

244 cernutum L.

245 chamaecyparissus A. Br.

246 clavatum L.

247 complanatum L.

248 inundatum $\mathrm{L}$.

249 Bigelovii Tuckerman

250 lucidulum Michx.

25 I obscurum I.

\section{(L. dendroideum Michx.)}

252 pinnatum (Chapm.) Lloyd \&

Underw.

253 porophilum Lloyd \& Underw.

254 sabinaefolium Willd.

255 Selago L.

256 Sitchense Rupr.

\section{Psilotum}

257 nudum ( $I_{\text {^.) }}$ Griseb.

\section{SELAGINELLACEAE}

\section{Selaginella}

258 apus (L.) Spring.

259 arenicola Underw.

260 Bigelovii Underw.

26I bryoides (Nutt.) Underw.

(S. cinerascens A. A. Eaton)

262 Douglasii. (T. \& G.) Spring.

263 lepidophylla Spring.

264 Ludoviciana A. Br.

265 mutica D. C. Eaton

266 Pringlei Baker

267 rupestris (L.) Spring.

268 Fendleri Underw.

269 rupincola Underw.

270 selaginoides (L.) Link

27 I struthioloides (Presl) Underw.

272 tortipila A. Br.

273 Watsoni Underw.

\section{ISOETACEAE}

\section{Isoetes}

274 Bolanderi Engelm.

275 Butleri Engelm.

276 Dodgei A. A. Eaton

277 Eatoni Dodge

278 echinospora Boottii Engelm.

279 Braunii (Durieu) Engelm.

280 muricata (Durieu) Engelm.

28I robusta Eingelm.

282 Engelmanni A. Br.

283 gracilis Engelm.

284 valida Engelm.

285 flaccida Shuttlew.

286 Chapmani Engelm.

287 foveolata A. A. Eaton

288 Howellii Fingelm.

(I. nuda, also I. Underwoodi)

289 lacustris L.

290 Macounii A. A. Eaton

29I maritima Underw.

292 melanopoda J. Gay

293 melanospora Fingelm.

294 minima A. A. Eaton

295 Nuttallii A. Br.

296 Orcutti A. A. Eaton

297 pygmaea Fingelm. 

298 riparia Engelm.

299 saccharata Engelm.

300 Tuckermani A. Br.

CYCADACEAE

\section{Zamia}

301 integrifolia Jacq.

302 pumila L.

\section{TAXACEAE}

\section{Tumion 1 \%}

(Torreya)

303 Californicum ('Torr.) Greene 304 taxifolium (Arn.) Greene

\section{Taxus 18}

305 brevifolia Nutt. 306 Floridana Nutt.

307 minor (Michx.) Britton (T. Canadensis Willd.)

\section{PINACEAE}

\section{Pinus 22}

308 albicaulis Engelm.

309 A pacheca Lemmon

3IO aristata Engelm.

3II Arizonica Engelm.

3I 2 attenuata Lemmon

(P. tuberculata Gord.)

3I3 Balfouriana Murray

3I4 cembroides Zucc.

3I5 Chihuahuana Engelm.

3 I6 clausa (Engelm.) Sarg.

3 I 7 contorta Dougl.

3r 8 Coulteri Lamb.

3I9 divaricata (Ait.) Du Mont.

(P. Banksiana Lamb.)

320 echinata Mill.

(P. mitis Michx.)

$32 \mathrm{I}$ edulis Engelm.

322 flexilis James

323 megalocarpa Sudw.

324 glabra Walt.

325 heterophylla (E1l.) Sudw.

(P. Cubensis Griseb.)

326 Jeffreyi Oreg. Com.

327 Lambertiana Dougl.
328 Mayriana Sudw.

329 monophylla Torr. \& Frem.

330 monticola Dougl.

33I muricata Don.

332 Murrayana Oreg. Com.

333 palustris Mill.

334 ponderosa Dougl.

335 pungens Michx. f.

336 quadrifolia Parl.

(P. Parryana Fingelm.)

337 radiata Don

$33^{8}$ resinosa Ait.

339 rigida Mill.

340 Sabiniana Dorgl.

34I scopulorum (Engelm.) I,emmon

342 serotina Michx.

343 strobiformis Fingelm.

344 Strobus I.

345 Taeda L.

346 tenuis Lemmon

347 Torreyana Parry

348 Virginiana Mill.

(P. inops Soland.)

\section{Larix $\mathbf{2 4}$}

349 laracina (Du Roi) Koch

(L. Americana Michx.)

350 Lyallii Parl.

35 I occidentalis Nutt.

\section{Picea 26}

352 brevifolia Peck

353 semiprostrata Peck

354 Breweriana Wats.

355 Columbiana Lemmon

356 Canadensis (L.) B. S. P. (P. alba Link.)

357 Engelmanni (Parry) Engelm.

$35^{8}$ Mariana (Mill.) B. S. P. (P. nigra Link)

359 Parryana (Andre) Parry (P.pungens Engelm.)

360 rubens Sargent

(P. rubra (Poir.) Dietr.)

36I Sitchensis (Bong.) T. \& M.

\section{Tsuga 27}

362 Canadensis (L.) Carr.

363 Caroliniana Engelm. 

364 heterophylla (Raf.) Sargent

(T.Mertensiana of authors, notCarr.) 365 Hookeriana (Carr.) Oreg. Com. 366 Mertensiana (Bong.) Carr. (T. Pattoniana Fingelm.)

\section{Pseudotsuga (2\%)}

367 macrocarpa (Torr.) Lemmon 368 mucronata (Raf.) Sudw.

369 elongata Lemmon

370 palustris (Raf.) Lemmon

37 I suberosa Lemmon.

\section{Abies 29}

372 amabilis (Loud.) Forbes

373 Arizonica Merriam

374 balsamea (Li:) Mill.

375 concolor (Gord.) Parry

376 Fraseri (Pursh) Lindl.

377 grandis Lindl.

378 lasiocarpa (Hook.) Nutt.

379 Lowiana Murray

380 magnifica Murray

$38 \mathrm{r}$ nobilis Lindl.

382 Shastensis Lemmon

383 venusta (Dougl.) Koch

\section{Sequoia 32}

384 sempervirens (Lamb.) Endl. 385 Washingtoniana (Winsl.) Sudw (S. gigantea Dec.)

\section{Taxodium $\mathbf{3 5}$}

386 distichum (L.) L. C. Rich.

\section{Libocedrus 41}

387 decurrens Torr.

\section{Thuja 42}

388 occidentalis L.

389 plicata Don

\section{Cupressus 43}

390 Arizonica Greene

39I Goveniana Gord.

392 Guadalupensis Wats.

393 Macnabiana Murray

394 macrocarpa Hartweg
Chamaecyparis 44

395 Lawsoniana (Murray) Parl.

396 Nootkatensis (Lamb.) Spach

397 thyoides (L.) B. S. P.

(C. sphaeroidea Spach)

\section{Juniperus 45}

398 Barbadensis L.

399 Californica Carr.

400 communis L.

401 flaccida Schlecht.

402 Knightii Aven Nelson

403 monosperma (Engelm.) Sargent

404 occidentalis Hook.

405 conjugens Engelm.

406 pachyphloea Torr.

407 prostrata Pers.

408 Sabina L.

409 sabinoides (H. B. K.) Sargent.

4IO scopulorum Sargent

4II Sibirica Burgsdorff (J. nana Willd.)

4I 2 Utahensis (Engelm.) L,emmon 4I3 Virginiana L.

\section{GNETACEAE}

\section{Ephedra 46}

4I4 antisyphilitica C. A. Meyer

4I 5 Californica Wats.

4 I6 Nevadensis Wats.

4I 7 pedunculata Engelm.

4I 8 Torreyana Wats.

419 trifurca Torr.

420 viridis Coville

\section{TYPHACEAE}

\section{Typha 49}

$42 \mathrm{I}$ angustifolia $\mathrm{L}$.

422 Domingensis Pers.

423 latifolia L.

\section{SPARGANIACEAE}

\section{Sparganium $\mathbf{5 4}$}

424 androcladum (Fingelm.) Morong

425 fluctuans Morong

(S. simplex var. fluitans)

426 eurycarpum Engelm. 

427 Greenei Morong

428 hyperboreum Laest.

429 minimum Fries

430 natans L.

43 r simplex Huds.

432 angustifolium (Michx.)

Engelm. 465 latifolius (Robbins) Morong

433 multipedunculatum Morong

\section{POTAMOGETONACEAE}

\section{Zostera $\mathbf{5 5}$}

434 latifolia Morong

(Z. Pacifica Wats.)

435 marina L.

436 minor (Carol.) Nolte

437 Oregana Wats.

\section{Phyllospadix 56}

438 Scouleri Hook.

439 Torreyi Wats.

\section{Potamogeton 58}

440 alpinus Balbis

(P. nufescens Schrad.)

44I amplifolius Tuckerman

442 confervoides Reichb.

(P. Tuckermani Robbins)

443 crispus L.

444 Curtissii Morong

445 diversifolius Raf.

( $P$. hybridus Michx.)

446 multidenticulatus Morong

447 trichophyllus Morong

448 Faxoni Morong

449 filiformis Pers.

450 occidentalis (Robbins)

45I Macounii Morong

Morong

452 foliostus Raf.

(P. panciflorus Pursh)

453 Californicus Morong

454 Niagarensis ('Tuckerman)

Morong

455 Friesii Rupr.

$45^{6}$ gemmiparus (Robbins) Morong

457 heterophyllus Schreb.

$45^{8}$ graminifolius (Fries)

Morong 497 occidentalis Wats.

459 minimus Morong
460 myriophyllus (Robbins)

46I Hillii Morong

Morong

462 Illinoensis Morong

463 interruptus Kitaibel

464 lateralis Morong

466 lonchites Tuckerman

(P. fuitans Roth)

467 Noveboracensis Morong

468 lucens L.

469 Connecticutensis Robbins

470 marinus L.

47 I mucronatus Schrad.

472 Mysticus Morong

473 natans $L$.

474 Nuttallii C. \& S.

(P. Pennsylvanicus Cham.)

475 Cayugensis Wiegand.

476 Oakesianus Robbins

477 obtusifolius Mert. \& Koch

478 pectinatus $\mathrm{L}$.

479 perfoliatus I.

480 Richardsonii A. Bennett

( $P$. perfoliatus var. lanceolatus)

48 I praelongus Wulf.

482 pulcher Tuckerman.

483 pusillus L.

484 Panormitanus (Biv.)

485 polyphyllus Morong 486 Sturrockii A. Bennett 487 Robbinsii Oakes

488 rutilus Wolfg.

489 spathulaeformis (Robbins)

490 Spirillus Tuckerman

Morong

49I Vaseyi Robbins

492 Zizii Roth

493

Methyensis (A. Bennett)

Morong

494 zosteraefolius Schum.

\section{Ruppia $\mathbf{5 9}$}

495 curvicarpa Aven Nelson

496 maritima L.

498 pectinata Rydb. 

Zannichellia $6 \%$

499 palustris L.

500 muricata Morong

501 pedunculata A. Gray

\section{NAIADACEAE}

\section{Naias 64}

502 flexilis (Willd.) Rost. \& Schm. 503 robusta Morong 504 gracillima (A. Br.) Morong 505 Guadalupensis (Spreng.)

506 marina L.

507 gracilis Morong 508 recurvata Dudley 509 microdon A. Br.

JUNCAGINACEAE

\section{Triglochin 66}

5Io concinna Davy

5II maritima L.

5I 2 palustris $\mathrm{L}$.

5I3 striata R. \& P.

Scheuchzeria 6\%

5I4. palustris $\mathrm{I}_{4}$.

\section{Lilaea 69}

5I5 subulata H. B. K.

\section{ALISMACEAE}

\section{Alisma 70}

5I6 Plantago-aquatica I.

5I7 tenellum Mart.

5I 8 validum Greene

\section{Damasonium 74}

519 Californicum 'Torr.

Echinodorus 75

520 cordifolius (L.) Griseb.

(E. rostratus Engelm.)

521 parvulus Engelm.

522 radicans (Nutt.) Engelm.

\section{Lophotocarpus $\quad \mathbf{7 6}$}

523 calycinus (Engelm.)

J. G. Smith

(Sagittaria calycina Fingelm.)

\section{Sagittaria 78}

524 ambigua J. G. Smith

525 arifolia Nutt.

526 stricta J. G. Smith

527 Chapmani (J. G. Smith) Mohr

528 Chinensis Sims

529 cristata Engelm.

530 cuneata Sheldon

53 I cycloptera (J. G. Smith) Mohr

532 Engelmanniana J. G. Smith

(S. variabilis var. gracilis)

533 filiformis J. G. Smith

534 Geyeri Torr.

535 graminea Michx.

536 Greggii J. G. Smith

537 lancifolia L.

538 falcata (Pursh) J. G. Sinith

539 latifolia Willd.

(S. variabilis Engelm.)

540 pubescens (Muhl.)

54I longiloba Engelm.

542 longirostrata (Michx.)

J. G. Smith

J. G. Smith

543 australis J. G. Smith

544 macrocarpa J. G. Smith

545 Mohrii J. G. Smith

546 Montevidensis C. \& S.

547 papillosa Buchenau

548 platyphylla (Engelm.)

549 recurva Fingelm.

J. G. Smith

550 rigida Pursh

(S. heterophylla Pursh)

55I Sanfordii Greene

552 subulata (L.) Buchenau

553 gracillima (Wats.)

J. G. Smith

554 natans (Michx.) J. G. Smith

555 teres Wats.

556 viscosa Mohr.

\section{HYDROCHARITACEAE}

Halophila 85

557 Engelmanni Asch.

\section{Philotria 8\%}

(Elodea)

$55^{8}$ Canadensis (Michx.) Britton 

Vallisneria $\mathbf{8 9}$

559 spiralis L.

\section{Limnobium 97}

560 Spongia (Bosc.) L. C. Rich.

\section{GRAMINEAE}

\section{Tripsacum 103}

$56 \mathrm{I}$ dactyloides $\mathrm{L}$. 562 Floridanum Porter 563 Lemmoni Vasey

\section{Coix $10 \%$}

564 lachryma-Jobi L.

\section{Imperata 109}

565 Brasiliensis 'Trin.

566 Hookeri Rupr.

\section{Erianthus 112}

567 alopecuroides (L.) Ell1.

568 brevibarbus Michx.

569 compactus Nash

570 contortus Ell.

57 I laxus Nash

572 saccharoides Michx.

573 Smallii Nash

574 strictus Baldwin

575 Tracyi Nash

\section{Manisurus 12\%}

(Rottboellia)

576 compressa (L. f.) Kuntze

577 cylindrica (Michx.) Kuntze

578 rugosa (Nutt.) Kuntze

579 tesselata (Stend.) Scribn.

580 tuberculosa Nash

\section{Hackelochloa 1\%8}

(Manisurus)

$5^{8 \mathrm{I}}$ granularis (L.) Kuntze

\section{Trachypogon 131}

582 polymorphus Montfari

\section{Elionurus 13\%}

(H. B. K.) Hack.

583 barbiculmis Hack.

584 tripsacoides H. B. K.

585 ciliaris (Trin.) Beal

\section{Andropogon 134}

586 arctatus Chapm.

587 argyraeus Schult.

(A. argentaitus Fill.)

588 brachystachys Chapm.

589 campyloracheus Nash

(A. Elliottii var. laxiflorus)

590 Cabanisii Hack.

59I capillipes Nash

(A. Virginicus var. glaucus)

592 cirratus Hack.

593 contortus L.

594 corymbosus (Chapm.) Nash

595 Elliottii Chapm.

596 Floridanus Scribn.

597 furcatus Mulul.

598 geminatus Hack.

599 glaucopsis Fill.

600 glomeratus (Walt.) B. S. P.

(A. macrourus Michx.)

601 gracilis Spreng.

602 gyrans Ashe

603 Hallii Hack.

604 hirtiflorus Kunth

605 Liebmanii Hack.

606 longiberbis Hack.

607 maritimus Chapm.

608 Mohrii Hack.

609 Pungensis Ashe

61o Neo-Mexicanus Nash

6I I oligostachys Chapm.

$6 \times 2$ saccharoides Sw.

6I 3 scoparius Michx.

614 Scribnerianus Nash

6I 5 semiberbis (Nees) Kunth

6I6 tener Kunth

6I7 Tennesseensis Scribn.

6r8 tetrastachyus E1l.

6r9 Torreyanus Steud.

620 Tracyi Nash

621 Virginicus L.

622 Wrightii Hack.

\section{Sorghum (134)}

623 Halapense (L。) Pers.

Chrysopogon (134)

624 avenaceus (Michx.) Benth. 

625 nutans (L.) Benth.

626 pauciflorus (Chapm.) Nash 627 secundus Benth.

\section{Heteropogon (134)}

628 contortus (L.) Beauv. 629 melanocarpus (E11.) Benth.

\section{Anthephora 140}

630 elegans Schreb.

\section{Hilaria 141}

63 I cenchroides H. B. K. $63^{2}$ Texana Vasey

633 Jamesii (Torr.) Benth. 634 inutica (Buckley) Benth. 635 rigida (Thurb.) Scribn.

\section{Aegopogon 142} 636 cenchroides H. \& B. 637 geminiflorus H. B. K.

\section{Nazia 143}

(Tragus)

638 aliena (Spreng.) Sçribn.

(T. occidentalis Nees) 639 racemosa (L.0) Kuntze

\section{Reimaria 160}

640 oligostachya Munro

\section{Paspalum 161}

64I bifidum (A. Bertol.) Nash (P. racemulosum Nutt.)

642 Blodgettii Chapm.

643 Boscianum Fluegge (P. purpurascens E:11.)

644 Buckleyanum Vasey

645 Chapmani Nash

646 ciliatifolium Michx.

647 compressum (Sw.) Nees

648 conjugatum Berg.

649 Curtisianum Steud.

650 difforme LeConte

65 I dilatatum Poir.

652 distichum L.

653 Eggertii Nash

654 elatum Rich.

655 Floridanum Michx.

656 geminum Nash
657 giganteum Baldwin

$65^{8}$ glabratum (Engelm.) Mohr

659 Hallii Vasey \& Scribn.

(P.pubiflorum glaucum Scribn)

660 laeve Michx.

66I lividum Trin.

662 longicilium Nash

663 longipedunculatum LeConte

664 longipilum Nash

665 membranaceum Walt.

(P. Walterianum Schultes)

666 monostachyum Vasey

667 mucronatum Muh1.

( $P$. fluitans Kunth)

668 paspaloides (Michx.) Scribn. (P. Elliottii Wats.)

669 plicatulum Michx.

670 praecox Walt.

67 I propinquum Nash

672 pubiflorum Rupr.

673 rigidifolium Nash

674 scrobiculatum L.

675 setaceum Michx.

676 Simpsoni Nash

677 villosissimum Nash

678 virgatum pubiflorum Vasey

\section{Anthaenantia 16\%}

679 rufa (E11.) Schultes

680 villosa Beauv.

\section{Amphicarpon 163}

681 Amphicarpon (Pursh) Nash (Amphicarpum Purshii Kunth)

682 Floridanum (Chapm.) Beal

\section{Eriochloa 164}

683 Lemmoni Vasey \& Scribn.

684 longifolia Vasey

685 mollis Kunth

686 punctata (L.) W. Hamilt.

(E. polystachya H. B. K.)

687 sericea Munro

\section{Panicum 166}

688 Addisonii Nash

689 agrostidiforme Lam.

690 agrostoides Muhl.

69I albomarginatum Nash 

692 amarum Ell.

693 angustifolium El1.

694 annulum Ashe

695 arenicola Ashe

696 Ashei Pearson

697 Atlanticum Nash

698 antumnale Bosc.

699 Baldwinii Nutt.

700 barbipulvinatum Nash

7or barbulatum Michx.

702 Bicknellii Nash

703 boreale Nash

704 brachyanthum Steud.

705 Brittonii Nash

706 bulbosum H. B. K.

707 Bushii Nash

708 Cahoonianum Ashe

709 calliphyllum Ashe

7 Io capillare L.

7II Gattingeri Nash

7 I 2 capillarioides Vasey

7I3 Chapmani Vasey

7I4 ciliatissimum Buckley

7 I 5 ciliatum Ell.

7 I6 ciliiferum Nash

7 I 7 ciliosum Nash

7 I8 clandestinum L.

7 I9 Clutei Nash

720 cognatum Schultes

721 Columbianum Scribn.

722 Commonsianum Ashe

723 commutatum Schultes

724 consangtuineum Kunth

7.25 Currani Ashe

726 curtifolium Nash

727 decoloratum Nash

728 depauperatum Muhl.

729 dichotomum L.

730 digitarioides Carpenter

(P. Curtisii)

73I Earlei Nash

732 Eatoni Nash

733 elongatım Pursh

734 ensifolium Baldwin (P. Cuthbertii Ashe)

735 epilifolium Nash

736 equilaterale Scribn.

737 erectifolium Nash
$73^{8}$ fasciculatum Sw.

739 filiculme Ashe

740 filipes Scribn.

74 I flavovirens Nash

742 flexile (Gattinger) Scribn.

743 gibbum Ell.

744 glabrifolium Nash

745 glabrissimum Ashe

746 gymnocarpon Ell.

747 Hallii Vasey

748 Havardi Vasey

749 Helleri Nash

750 hians Ell.

75 I hirticaulum J. S. Presl

752 Huachucae Ashe

753 implicatum Scribn.

754 inflatum Scribn. \& Smith

755 insulare (L.) Meyer

(P. leucophaeum H. B. K.)

756 Joorii Vasey

757 lanatum Rottb.

$75^{8}$ lanuginosum E11.

759 latifolium L.

760 laxiflorum Lam.

76I Leibergii (Vasey) Scribn.

762 lencothrix Nash

763 Lindheimeri Nash

764 linearifolium Scribn.

765 longifolium Torr.

766 longiligulatum Nash

767 longipedunculatum Scribn.

768 lucidum Ashe

769 macrocarpon LeConte

770 maculatum Ashe

77 I malacon Nash

772 malacophyllum Nash

773 Manatense Nash

774 Mattamusketense Ashe

775 maximum Jacq.

776 meridionale Ashe

777 microcarpon Muhl.

778 microphyllum Ashe

779 miliaceum L.

780 minus (Muhl.) Nash

78 r molle Sw.

782 neuranthum Griseb.

783 Nashianum Scribn.

784 nemopanthum Ashe 

785 nitidum Lam.

786 nudicaule Vasey

787 obtusum H. B. K.

788 occidentale Scribn.

789 octonodum Scribn. \& Smith

790 Orangense Ashe

79 I ovale Ell.

792 ovinum Scribn. \& Smith

793 parvispiculum Nash

794 paspaloides Pers.

795 patentifolium Nash

796 pauciflorumh Eill.

797 paucipilum Nash

798 pedicellatum Vasey

799 pernervosum Nash

800 perlongum Nash

8or platyphyllum Munro

802 polyanthes Schultes

803 polycaulon Nash

804 Porterianum Nash

805 proliferum Lam.

806 prostratum Lam.

807 psammophilum Nash

808 pseudanceps Nash

809 pseudopubescens Nash

81o pubescens Lam.

8I I pubifolium Nash

$8 \mathrm{I} 2$ pyriforme Nash

$8 \mathrm{I}_{3}$ repens $\mathrm{L}$.

814 Reverchoni Vasey

815 Roanokense Ashe

816 rostratum Muhl. ( $P$. anceps Michx.)

8I7 saccharatum Buckley (P. lachnanthum A. Gray)

8I8 scabriusculm El1.

8I9 scoparioide Ashe

820 scoparium Lam.

821 Scribnerianum Nash

822 sparsiflorum Vasey

823 sphaerocarpon Ell.

824 sphagnicola Nash

825 stenodes Griseb.

826 strictifolium Nash

827 subspicatum Vasey

828 tenerrimum Trin.

829 Tennesseense Ashe

830 Texanum Buckley
83 I thermale Bolander

832 Thurowii Scribn. \& Smith

833 trifolium Nash

834 tsugetorum Nash

835 Urvilleanum Kunth

836 verrucosum Muhl.

837 villosissimum Nash

838 virgatum $L$.

839 breviramosum Nash

840 confertum Vasey

$84 \mathrm{I}$ viscidum Ell.

842 Webberianum Nash

843 Werneri Scribn.

844 Wilcoxianum Vasey

845 Wrightianum Scribn.

846 xanthophysum A. Gray

\section{Echinochloa (166)}

(Panicum in part)

847 colona (L.)

848 Crus-galli (L.) Beauv.

849 Walteri (Pursh)

\section{Syntherisma (166)}

(Panicum in part)

850 Bakeri Nash

$85 \mathrm{I}$ barbata (Willd.) Nash

852 filiformis (L.) Nash.

853 fimbriata (Link) Nash

854 fuscescens (Presl) Scribn.

855 gracillima (Scribn.) Nash

856 humifusa (Pers.) Rydb.

(S. linearis (Krock) Nash)

857 Mississippiensis (Gattinger)

858 leucocoma Nash

859 sangrinalis (L.) Dulac

860 serotina Walt.

86I setosa (Desv.) Nash

862 setigera (Roth) Nash

863 Simpsoni (Vasey) Nash

864 villosa Walt.

\section{Oplismenus 169}

865 hirtellus (L/.) R. \& S.

866 Liebmanii Fourn

Chaetochloa $1 \% 1$

(Setaria, also Ixophorus, Nas1.) 

867 ambigua (Guss.) S. \& M.

868 brevispica S. \& M.

869 caudata (Lam.) Scribn.

870 composita (H. B. K.) Scribn.

871 corrugata (Ell.) Scribn.

872 parviflora (Poir.) S. \& M.

873 flava (Nees) Scribn.

874 gibbosa S. \& M.

875 glauca (L.) Scribn.

876 gracilis (H. B. K.) S. \& M.

877 Grisebachii (Fourn.) Scribn.

878 ampla S. \& M.

879 imberbis (Poir.) Scribn.

870 penicillata (Nees) S. \& M.

88I Italica (I.) Scribn.

882 Germanica (Mill.) Scribn.

883 macrosperma S. \& M.

(Setaria composita of Chapm.)

884 macrostachya (H.B. K.) S. \& M.

885 magna (Griseb.) Scribn.

886 pauciseta Vasey

887 perennis (Curtiss) Bicknell

888 polystachya (Scheele) S. \& M.

889 purpurascens (H. B. K.)

880 setosa (Sw.) Scribn.

891 versicolor Bicknell

892 verticillata (L.) Scribn.

893 villosissima S. \& M.

894 viridis (L.) Scribn.

\section{Cenchrus 1\%4}

895 echinatus L.

896 gracillimus Nash

897 incertus M. A. Curtis

898 macrocephalus (Doell) Scribn.

899 myosuroides H. B. K.

900 tribuloides $\mathrm{L}$.

\section{Pennisetum 1\%5}

901 setosum (Sw.) Rich.

Stenotaphrum 180

902 secundatum (Walt.) Kuntze (S. Americanum Schranck)

\section{Hydrochloa 185}

903 fluitans (Michx.) Torr.
Pharus 186

904 latifolius L.

\section{Luziola 188}

905 Alabamensis Chapm.

906 Peruviana J. F. Gmel.

\section{Zizaniopsis 189}

907 miliacea (Michx.) D. \& A. (Zizania miliacea Michx.)

\section{Zizania 190}

908 aquatica L.

\section{Homalocenchrus 194}

(Leersia)

909 hexandra (R. \& S.) Britton

900 lenticularis (Michx.) Scribn.

9I I monandra (R. \& S.) Britton

9I 2 oryzoides (L.) Poll.

93 Virginicus (Willd.) Britton

\section{Phalaris $\mathbf{2 0 4}$}

9I4 amethystina Trin.

9I5 angusta Nees

9I6 arundinacea L.

9I7 Canariensis L.

9I8 Caroliniana Walt. ( $P$. intermedia Bosc.)

919 Lemmoni Vasey

\section{Anthoxanthum $\mathbf{2 0 5}$}

920 odoratum I.

92 I Puelii Lecoq. \& Lamotte

Savastana $\mathbf{2 0 6}$

(Hierochloe)

922 alpina (Sw.) Scribn.

923 macrophylla ('Thurb.) Beal

924 Nashii Bicknell

925 odorata (L.) Scribn.

(Hierochloe borealis R. \& S.)

926 pauciflora (R. Br.) Scribn.

Aristida 208

927 Arizonica Vasey

928 basiramea Vasey

929 Californica Thurb.

930 fugitiva Vasey 

93 I condensata Chapm.

932 desmantha Trin. \& Rupr.

933 dichotoma Michx.

934 Curtissii A. Gray

935 divergens Vasey

936 fasciculata Torr.

(A. dispersa Trin. \& Rupr.)

937 Floridana (Chapm.) Vasey

938 gracilis Ell.

939 gyrans Chapm.

940 Havardii Vasey

94t Humboldtiana Trin. \& Rupr.

942 lanosa Muhl.

(A. lanata Poir.)

943 longiseta Steud.

944 Mohrii Nash

945 Nealleyi Vasey

946 oligantha Michx.

947 nervata Beal

948 Orcuttiana Vasey

949 Palmeri Vasey

950 palustris Vasey

95 I patula Chapm.

952 purpurascens Poir.

953 purpurea Nutt.

954 ramosissima Engelm.

955 Reverchoni Vasey

956 scabra Kunth

957 Schiediana Trin. \& Rupr.

$95^{8}$ minor Vasey

959 simpliciflora Chapm.

960 spiciformis Ell.

96I stricta Michx.

962 tuberculosa Nutt.

963 virgata Trin.

\section{Stipa 209}

964 Andersoni Vasey

965 avenacea $L$.

966 avenacioides Nash

967 Bloomeri Bolander

968 comata Trin. \& Rupr.

969 coronata Thurb.

970 Elmeri Piper \& Brodie

(S. viridula var. pubescens)

971 eminens Cav.

972 flexuosa Vasey

973 Hassei Vasey
974 Kingii Bolander

975 Lettermani Vasey

976 Macounii Scribn.

977 minor (Vasey) Scribn.

978 Mongolica Turcz.

979 Nelsonii Scribn.

980 Neo-Mexicana('Thurb.)Scribn.

98I occidentalis Thurb.

982 Oregonensis Scribn. (S. stricta Vasey, not Lam.)

983 Parishii Vasey

984 Pringlei Scribn.

985 Richardsonii Link.

986 Scribneri Vasey

987 setigera Presl

988 Sibirica Lam.

989 spartea Trin.

990 speciosa Trin. \& Rupr.

99I tenuissima Trin.

992 Tweedyi Scribn.

993 Vaseyi Scribn.

994 viridula Trin.

995 Williamsii Scribn.

\section{Oryzopsis $\mathbf{2 1 0}$}

996 asperifolia Michx.

997 exigua 'Thurb.

998 fimbriata Hemsl.

999 Hendersoni Vasey

Iooo juncea (Michx.) B. S. P.

(O. Canadensis Torr.)

IOoI melanocarpa Muhl.

IOO2 micrantha (Trin. \& Rupr.)

Thurb.

IOO3 miliacea (L.) Davy

\section{Eriocoma (210)}

IOO4 caduca (Scribn.) Rydb. (Stipa caduca Scribñ.)

IOO5 cuspidata Nutt.

I006 Webberi Thurb.

\section{Milium 213}

1007 effusum L.

\section{Muhlenbergia}

215

Ioo8 Alamosae Vasey

roog ambigua Torr.

roIo arenicola Torr. 

IOI I Arizonica Scribn.

ror 2 Berlandieri Trin.

IOI3 Californica Vasey

IOI4 capillaris (Iam.) Trin.

Ior 5 comata ('Thurb.) Benth.

ror6 diffusa Schreb.

Ior7 distichophylla (Presl) Munro

Ior 8 mutica Scribn.

ror9 dumosa Scribn.

IO2O filiculmis Vasey

IO2I filipes M. A. Curtis

IO22 gracilis H. B. K.

IO23 gracillima Torr.

I024 Lemmoni Scribn.

ro25 Mexicana (L.) Trin.

1026 microsperma (DC.) Trin.

I027 monticola Buckley

ro28 Neo-Mexicana Vasey

ro29 palustris Scribn.

ro3o parviglumis Vasey

IO3I pauciflora Vasey

1032 Porteri Scribn.

I033 Pringlei Scribn.

I034 pungens Thurb.

I035 racemosa (Michx.) B. S. P.

(M. glomerata Trin.)

1036 Schaffneri Fourn.

I037 sobolifera (Muhl.) Trin.

I038 sylvatica Torr.

I039 Californica Vasey

I040 gracilis Scribn.

IO4 $\mathrm{I}$ setiglumis Wats.

IO42 tenuiflora (Willd.) B. S. P.

(M. Willdenovii Trin.)

ro43 trichopodes E11.

ro44 Vaseyana Scribn.

I045 virescens (H. B. K.) Trin.

I046 Wrightii Vasey

\section{Brachyelytrum $\mathbf{2 1 6}$}

ro47 erectum (Schreb.) Beauv. (B. aristatum Beauv.)

\section{Lyeurus 218}

I048 phalaroides H. B. K. ro49 phleoides H. B. K.

\section{Heleochloa 22\%}

ro5o schoenoides (L.) Host.

\section{Phleum 223}

ro5I alpinum L.

ro52 pratense I.

\section{Alopecurus $\mathbf{2 2 5}$}

I053 agrestis I.

I054 alpinus J. E. Smith

I055 Californicus Vasey

I056 geniculatus L.

I057 fulvus (J. E. Smith) Scribn

I058 robustus Vasey

I059 Howellii Vasey

I060 Merrimani Beal

106I occidentalis Scribn. \& Tweedy

ro62 pratensis L.

I063 saccatus Vasey

\section{Coleanthus 228}

I064 subtilis Seid

\section{Phippsia 2\%9}

I065 algida (Soland.) R. Br.

\section{Sporobolus $\mathbf{2 3 0}$}

I066 airoides Torr.

I067 argutus (Nees) Kunth

I068 asper (Michx.) Kunth

I069 asperifolius (Nees \& Meyen)

Thurb.

ro7o auriculatus Vasey

ro7 I Bolanderi Vasey

I072 brevifolius (Nutt.) Scribn.

I073 Buckleyi Vasey

I074 compressus ('Torr.) Kunth

I075 confusus Vasey

ro76 cryptandrus (Torr.) A. Gray

I077 flexuosus Thurb.

I078 strictus Scribn.

ro79 Curtissii (Vasey) Small

ro8o cuspidatus ('Torr.) Wood.

I08I depauperatus (Torr.) Scribn.

I082 Domingensis ('Trin.) Kunth

I083 filiculmis (Thurb.) Vasey

ro84 filiformis (Thurb.) Rydb.

I085 Floridanus Chapm.

I086 giganteus Nash

I087 gracillimus (Thurb.) Vasey

I088 heterolepis A. Gray

I089 Indicus (I.) R. Br. 

rogo inflatus Vasey \& Dewey

I09I interruptus Vasey

I092 Jonesii Vasey

I093 junceus (Michx.) Kunth

I094 ligulatus Vasey \& Dewey

ro95 longifolius (Torr.) Wood.

rog6 Nealleyi Vasey

I097 neglectus Nash

I098 pilosus Vasey

1099 purpurascens Hamilton

I IOO Sacatilla Griseb.

I IOI serotinus (Torr.) A. Gray

I IO2 simplex Scribn.

I Io3 Texanus Vasey

II04 Thurberi Scribn.

I I05 utilus Torr.

I 106 vaginaeflorus ('Torr.) Wood (S. minor Vasey)

I 107 Virginicus (L.) Kunth

I I08 Wolfii Vasey

Irog Wrightii Scribn.

\section{Blepharonemron (230)}

I I ro tricholepis ('Torr.) Nash (Sporobolus tricholepis Coult.)

\section{Epicampes 231}

IIII rigens Benth.

\section{Polypogon 233}

III 2 elongatus (Poir.) H. B. K.

II 3 littoralis Sw.

II 4 maritimus Willd.

I I 5 Monspeliensis (L.) Desf.

\section{Limmodia 238}

(Thurberia)

IIr6 Arkansana (Nutt.)

\section{Arctagrostis $\mathbf{2 4 0}$}

I I 7 arundinacea ('Trin.) Beal

I I 8 latifolia (R. Br.) Griseb.

\section{Cinna 241}

III9 arundinacea L.

I 20 Bolanderi Scribn.

Ir 2 I latifolia (Trev.) Griseb.

(C. pendula Trin.)

II22 glomerata Scribn.

\section{Agrostis $\mathbf{2 4 \%}$}

I 23 aequivalvis Trin.

I 24 alba L.

I 25 altissima (Walt.) Tuckerman (A. elata Trin.)

II 26 asperifolia Trm.

I 27 attenuata Vasey

I 28 canina L.

I 29 stolonifera Vasey

I 30 densiflora Vasey

I 3 I Diegoensis Vasey

I 32 Elliottiana Schult. (A. arachnoides Ell.)

I 33 exarata Trin.

I 34 exigua Thurb.

I 35 geminata Trin.

I 36 grandis Trin.

I 37 Hallii Vasey

II 38 Howellii Scribn.

I 39 humilis Vasey

I 40 hyemalis (Walt.) B. S. P. (A. scabra Willd.)

Ir4I Idahoensis Nash

I 42 inexpansa Nash

I 43 inflata Scribn.

I 44 intermedia Scribn.

I 45 microphylla Steud.

I 46 Nova-Angliae Tuckerman

I 47 Nutkaensis Kunth

I 48 Oregonensis Vasey

I 49 paludosa Scribu.

I I 50 perennans (Walt.) Tuckerman

I 5 I rubra $\mathrm{L}$.

I 52 Sconleri Trin.

I 53 tenuiculmis Nash

(A. tenuis Vasey, not Sibth.)

I 54 recta Nash

I 55 variabilis Rydb.

(A. varians Trin.)

I 56 verticillata Vill.

I 57 virescens $H . B . K$.

\section{Gastridium $\mathbf{2 4 5}$}

I 58 australe Beauv.

Calamagrostis 247

I 59 Alaskana Kearney

II 60 Alentica Bong.

II6I patens Kearney 

I 62 Americana (Vasey) Scribn.

II63 angusta (Vasey) Kearney

I 64 blanda Beal

I 65 Bolanderi Thurber

II66 breviseta (A. Gray) Scribn.

(C. Pickeringii A. Gray)

II67 debilis Kearney

II68 lacustris Kearney

I 69 Breweri Thurber

I 70 Californica Kearney

I 7 I Canadensis (Michx.) Beauv.

I 72 acuminata Vasey

II 73 campestris Kearney

I 74 cinnoides (Muhl.) Bart.

(C. Nuttalliana Steud.)

II 75 confinis (Willd.) Nutt.

[This species has been mistaken for the European $C$. Lapponica Trin.]

I 76 crassiglumis Thurber

I 77 Cusickii Vasey

I 178 deschampsioides Trin.

I 79 fasciculata Kearney

II 80 foliosa Kearney

I 8 I Howellii Vasey

I182 hyperborea Lange

II 83 elongata Kearney

II84 stenodes Kearney

II85 inexpansa A. Gray

II86 barbulata Kearney

II 87 cuprea Kearney

I 88 koelerioides Vasey

II 89 densa (Vasey) Beal

I 190 Labradorica Kearney

I I9I lactea Beal

I 92 Langsdorfii (Link) Trin.

I 93 laxiflora Kearney

I 94 Lemmoni Kearney

I 95 Macouniana Vasey

II96 micrantha Kearney

I 97 montanensis Scribn.

I 98 neglecta (Ehrh.) Gaertn.

(C. stricta Beauv.)

I 99 borealis (Laest.) Kearney

I 200 candidula Kearney

I20I Wrightii Kearney

I 202 nemoralis Kearney

I 203 Porteri A. Gray
I 204 purpurascens R. Br.

I 205 arctica (Vasey) Kearney

I 206 rubescens Buckley

I 207 scopulorum Jones

I 208 lucida Kearney

I 209 Scribneri Beal

(C. dubia Scribn.)

I 2 ro subflexuosa Kearney

I 2 I I Suksdorfii Scribn.

I2I 2 luxurians Kearney

I 213 Tweedyi Scribn.

I 214 Vaseyi Beal

\section{Ammophila $\mathbf{2 4 9}$}

I 2 I 5 arenaria (L.) Link. (A. arundinacea Host.)

\section{Calamovilfa $\mathbf{2 5 0}$}

(Calamagrostis \& Calamovilfa)

I 216 brevipilis (Torr.) Hack.

I 2 I 7 Curtissii (Vasey) Scribn.

I 2 I 8 longifolia (Hook.) Hack.

\section{Apera $\mathbf{2 5 1}$}

I 2 I9 spica-venti (I/.) Beauv.

\section{Holeus 25y}

I 220 lanatus L.

\section{Aira $\mathbf{2 6 5}$}

I22 I caryophylla I.

I 222 praecox I.

\section{Deschampsia $\mathbf{2 \% 0}$}

I 223 atropurpurea (Wahl.) Scheele

I 224 brachyphylla Nash

I 225 caespitosa (L.) Beauv.

I 226 Bottnica (Wahl.) Vasey

I 227 longiflora Beal

I 228 maritima Vasey

1229 calycina Presl

1230 danthonioides (Trin.) Vasey

I 23I elongata (Hook.) Munro

I 232 flexuosa (L.) Trin.

I 233 gracilis Vasey

I 234 holciformis Presl

\section{Trisetum 271}

I235 argenteum Scribn.

I 236 barbatum Stend. 

I237 Brittonii Nash

I238 canescens Buckley

1239 cernuum Trin.

1240 elongatum (H. B. K.) Kunth

I 24 I flavescens (L.) R. \& S.

I 242 Hallii Scribn.

I243 Ludovicianum Buckley

I 244 montanum Vasey

I 245 Pennsylvanicum (L.) Beauv.

(T. palustre Torr.)

1246 subspicatum (L.) Beauv.

I247 molle (Michx.) A. Gray

\section{Avena $\mathbf{2 \% 3}$}

I248 Americana Scribn.

I 249 barbata Brot.

1250 fatua $L$.

I25I glabrescens Coss.

1252 Mortoniana Scribn.

I 253 sativa $\mathrm{L}_{\text {. }}$.

I254 Smithii Porter

I255 striata Michx.

\section{Arrhenatherum $\mathbf{2 \% 5}$}

I 256 elatius (L.) Beauv. (A. avenaceum Beauv.)

\section{Danthonia $\mathbf{2 8 0}$}

I257 Californica Bolander

$125^{8}$ compressa Austin

I 259 glabra Nash

1260 intermedia Vasey

126I Parryi Scribn.

I 262 sericea Nutt.

I 263 spicata (L.) Beauv.

I 264 unispicata Munro

\section{Capriola 28\%}

(Cynodon)

I 265 Dactylon (L.) Kuntze

\section{Spartina 283}

I 266 caespitosa A. A. Eaton I 267 cynosuroides (I.) Willd.

I 268 gracilis Trin.

I269 junciformis Engelm. \& Gray I270 patens (Ait.) Muhl.

(S. juncea E11.) •

127I polystachya (Michx.) Fill.
1272 stricta (Ait.) Roth.

1273 alterniflora (Lois.) A. Gray

I 274 maritima (Walt.) Scribn.

(S. stricta var. glabra)

\section{Campulosus $\mathbf{2 8 6}$}

\section{(Ctenium)}

1275 aromaticus (Walt.) Scribn.

(Ctenium Americanum Spreng) I 276 Chapadensis Trin.

\section{Chloris 288}

1277 barbata (L.) Nash

$$
\text { (C. polydactyla (L.) Sw.) }
$$

I 278 brevispica Nash

I 279 ciliata Sw.

I 28 o cucullata Bisch.

I 28I elegans H. B. K.

I 282 latisquamea Nash

I 283 Nealleyi Nash (C. Texensis Nash)

I 284 Prieuri Kunth

I 285 tenuispica Nash

I 286 Texana (Vasey) Nash

I 287 verticillata Nutt.

\section{Eustachys (288)}

(Chloris in part)

I 288 Floridana Chapm.

I 289 glauca Chapm.

I 290 neglecta Nash

I 29I petraea (Sw.) Desv.

\section{Trichloris $\mathbf{2 8 9}$}

I 292 fasciculata Fourn.

I 293 pluriflora Fourn.

\section{Gymnopogon 290}

I.294 ambiguus (Michx.) B. S. P.

(G. racemosus Beauv.)

I 295 brevifolius Trin.

\section{Schedommardus $29 \%$}

I 296 paniculatus (Nutt.) Trelease (S. Texanus Steud.)

\section{Bouteloua 295}

I 297 arenosa Vasey

I 298 aristidoides (Kunth) Griseb. 

I 299 breviseta Vasey.

I 300 bromoides (H. B. K.) Lag.

I 30 I Burkei Scribn.

r 302 curtipendula (Michx.) Torr. (B. racemosa Lag.)

I 303 eriopoda Torr.

I 304 Havardii Vasey

I 305 hirsuta Iag.

I 306 oligostachya (Nutt.) Torr.

I 307 polystachya (Benth.) Torr.

I308 prostrata Lag.

I 309 ramosa Scribn.

I3ro Rothrockii Vasey

I3 I T Texana Wats.

I 3 I 2 trifida Thurb.

I 3 I 3 uniflora Vasey

I 3 I 4 veslita (Wats.) Scribn.

Beckmannia $\mathbf{3 0 3}$

I 3 I 5 erucaeformis (L.) Host.

\section{Eleusine $\mathbf{3 0 4}$}

I3I6 Indica (L.) Gaertn.

Dactyloctenium $\mathbf{3 0 5}$

I 3I 7 Agyptiacum (L.) Willd.

\section{Leptochloa 30 ?}

I3I 8 Domingensis (Jacq.) Trin.

I3I9 Langloisii Vasey

I 320 mucronata (Michx.) Kunth

r32I pulchella Scribn.

I 322 Nealleyi Vasey

Bulbilis $\mathbf{3 0 8}$ (Buchloe)

I 323 dactyloides (Nutt.) Raf.

\section{Pappophorum $\mathbf{3 1 0}$}

I 324 apertum Munro

I 325 Wrightii Wats.

\section{Cottea 311}

I 326 pappophoroides Kunth

\section{Cathestecum $\mathbf{3 1 4}$}

I 327 erectum Vasey \& Hack.

r 328 Mexicanum Presl

Scleropogon $\mathbf{3 1 6}$

I 329 Karwinskianus (Fourn.) Benth I 354 flexuosa (Thurb.) Vasey
Monanthochloe $\mathbf{3 1 8}$

I330 littoralis Engelm.

Munroa 319

I33I squarrosa (Nutt.) Torr.

Orcuttia $3 \% 1$

I332 Greenei Vasey

\section{Arundo 331}

I333 Donax L.

\section{Phragmites 333}

I334 Phragmites (I.) Karst. (P. communis Trin.)

\section{Tricuspis (335)}

(Triodia in part.)

I335 albescens Munro

I 336 ambigua (Ell.) Chapm.

I337 Chapmani Small

I 338 congesta (I. H. Dewey)

I339 Drummondii Scribn. \&

I340 elongata (Buckley)

Kearney

I 34I eragrostoides Vasey \& Scribn.

I342 grandiflora Vasey

I343 Langloisii Nash

I344 mutica Torr.

I345 Nealleyi (Vasey)

I346 pilosa (Buckley)

I 347 pulchella (H. B. K.) Torr.

I348 sesleroides (Michx.) Torr.

(Triodia cuprea Jacq.)

I 349 stricta Nutt.

I350 Texana Thurb.

\section{Triplasis (335)}

(Triodia in part)

I35I Americana Beauv.

r $35^{2}$ purpurea (Walt.) Chapm.

(Triodia purpurea Hack.)

\section{Sieglingia (335)}

(Triodia in part)

I353 decumbens (L.) Kuntze

Redfieldia $\mathbf{3 3 6}$ 



\section{- Diplachne 33'}

I 355 dubia (Nees) Benth.

I 356 fascicularis (Lam.) Beauv.

I357 Halei Nash

I $35^{8}$ imbricata (Thurb.) Scribn.

I359 Pringlei (Beal) Nash

I 360 rigida (Munro) Vasey

I36I spicata Doell

I362 Tracyi Vasey

I 363 viscida Scribn.

\section{Dissanthelium 339}

I364 Californicum (Nutt.) Benth.

\section{Molinia $\mathbf{3 4 0}$}

I365 coerulea (L.) Moench.

\section{Eragrostis 341}

I366 Beyrichii J. G. Smith

I367 Brownei (Kunth) Nees

I368 capillaris (L.) Nees

I 369 ciliaris (I.) Link

r 370 curtipedicellata Buckley

I37 I Eragrostis (L.) Karst.

$$
\text { (E. minor Host.) }
$$

I372 Frankii Steud.

r 373 glomerata (Walt.)

L. H. Dewey

I374 grandiflora Smith \& Bush

r 375 hirsuta (Michx.) Nash

I 376 hypnoides (I.) B. S. P.

$$
\text { (E. reptans Nees) }
$$

I 377 lugens Nees

I378 lutescens Scribn.

I 379 major Host.

I380 Neo-Mexicana Vasey

I 38 I obtusiflora (Fourn.) Scribn.

I 382 pectinacea (Michx.) Steud.

I 383 pilosa (L.) Beauv.

\section{(E. Orcuttiana Vasey)}

I 384 plumosa Link

${ }^{1} 385$ Purshii Schrad.

I 386 refracta (Muhl.) Scribn. (E. campestris Trin.)

I387 secundiflora Presl

I 388 sessilispica Buckley

I389 sporoboloides Smith \& Bush

I 390 trichodes (Nutt.) Nash

(E. tenuis A. Gray)
I39I viscosa Scribn.

\section{Eatonia 344}

1392 filiformis (Chapm.) Vasey

I 393 longiflora (Vasey) Beal

I.394 nitida (Spreng.) Nash

(E Dudleyi Vasey)

I 395 obtusata (Michx.) A. Gray

I396 robusta Vasey

I397 Pennsylvanica (DC.) A. Gray

I 398 major 'Torr.

\section{Koeleria $\mathbf{3 4 6}$}

I 399 cristata (L.) Pers.

Catabrosa $\mathbf{3 4}$ \%

I 400 aquatica (L.) Beauv.

Anthochloa $\mathbf{3 5 3}$

(Stapfia)

I40I Colusana (Davy) Scribn.

\section{Melica $\mathbf{3 5 5}$}

I 402 aristata Thurb.

I 403 bromoides A. Gray

I 404 Howellii Scribn.

I 405 bulbosa Geyer

I406. Californica Scribn.

1407 diffusa Pursh

I408 nitens Scribn.

I 409 frutescens Scribn.

I4 Io fugax Bolander

I4I I Harfordii Bolander

I4I 2 imperfecta Trin.

I 4 I 3 flexuosa Bolander

I4I4 minor Scribn.

I4 5 refracta Thurb.

I4I 6 inflata Vasey

I4I 7 longiligula Scribn. \& Kearney

I4I 8 multinervosa Vasey

I4I9 mutica Walt.

I 420 parviflora (Porter) Scribn.

I42I spectabilis Scribn.

I422 stricta Bolander

I423 subulata (Bong.) Scribn.

(M. acuminata Bolander)

I424 Torreyana Scribn. 

Korycarpus $\mathbf{3 5 6}$

(Diarrhena)

I425 diandrus (Michx.) Kuntze

(Diarrhena Americana Beauv.)

\section{Pleuropogon 36:3}

I 426 Californicum (Nees) Benth.

I 427 refractum (A. Gray) Benth.

I428 Sabinii R. Br.

\section{Uniola 365}

I429 latifolia Michx.

I430 laxa (L.) B. S. P. (U. gracilis Michx.)

143 I longifolia Scribn.

I432 paniculata L.

I433 sessiliflora Poir.

(U. nitida Baldwin)

\section{Distichlis $\mathbf{3 6 6}$}

1434 spicata (L.) Greene

(D. maritima Raf.)

I435 stricta (Torr.) Scribn.

I436 Texana (Vasey) Scribn.

(Poa Texana and Sieglingia Wrightii Vasey)

\section{Briza 369}

I437 maxima L.

r 438 media L.

I439 minor L.

\section{Dactylis $3 \%$}

I440 glomerata L.

\section{Cynosurus $\mathbf{3 7 3}$}

I 44I cristatus I.

\section{Lamarckia $\mathbf{3 \% 4}$}

I442 aurea (L.) Moench.

\section{Poa 3 \% 8}

I443 abbreviata R. Br.

I444 acuminata Scribn.

I445 acutiglumis Scribn.

I446 alpicola Nash

I 447 alpina L.

I 448 alsodes A. Gray

r449 annua L.

I450 arachnifera Torr.
I45I glabrata Vasey

I452 arctica R. Br.

I 453 argentea Howell

I454 atropurpurea Scribn.

I455 autumnalis Muhl.

$$
\text { (P. Alexuosa Muhl.) }
$$

I456 Bigelovii Vasey \& Scribn.

I457 Bolanderi Vasey

I458 Brandegei Scribn.

I459 brevifolia Muhl.

I 460 brevipaniculata Scribn. \&

Williams

I46I subpallida Williams

I462 Buckleyana Nash

(P. tenuifolia Buckley)

I 463 caesia J. E. Smith

I464 capillaefolia Scribn. \&

I 465 cenisia All.

I466 Chapmaniana Scribn.

(P. cristata Chapm.)

I 467 compressa I.

I468 confinis Vasey

I469 curtifolia Scribn.

I 470 Cusickii Vasey

147 I debilis Torr.

I 472 Douglasii (H. \& A.) Nees

I473 Eatoni Wats.

I474 epilis Scribn.

I 475 Fendleriana (Steud.) Vasey

I 476 Arizonica Williams

I 477 spicata (Vasey) Scribn.

I 478 flava L.

(P. serotina Ehrh.)

I 479 flavicans Ledeb.

I 480 glauca Vahl.

r48I glaucifolia Scribn. \&

I482 glumaris Trin.

Williams

I483 gracillima Vasey

I484 Grayana Vasey

I485 Hanseni Scribn.

I486 hispidula Vasey

I 487 Howellii Vasey \& Scribn.

I488 Idahoensis Beal

I 489 incurva Scribn. \& Williams

I 490 invaginata Scribn. \& Williams

I49I juncifolia Scribn. 

I 492 Kaibensis Jones

I 493 Kelloggii Vasey

I 494 laevigata Scribn.

I 495 laxa Haenke

I 496 I,eckenbyi Scribn.

I 497 Leibergii Scribn.

I498 leptocoma Bong.

I499 Lettermani Vasey

I50o limosa Scribn. \& Williams

I 5 OI longepedunculata Williams

I5O2 longiligula Scribn. \&

Williams

I 503 Wyomingensis Williams .

I 504 longipila Nash

I 505 lucida Vasey

I 506 macrantha Vasey

I 507 nemoralis $\mathrm{L}$.

I 508 nervosa (Hook.) Vasey

I 509 Nevadensis Vasey

I5IO nudata Scribn.

I 5 I I occidentalis (Vasey) Rydb.

I 5I 2 Orcuttiana Vasey

I 513 Pattersoni Vasey

I 5 I 4 pratensis L.

I 5 I 5 pratericola Rydb. \& Nash

I 516 Pringlei Scribn.

I5I7 pseudopratensis Scribn. \&

I5I 8 purpurascens Vasey

I 5 9 reflexa Vasey \& Scribn.

I520 rupicola Nash

$$
\text { (P. mpestris Vasey) }
$$

I52I Sandbergii Vasey

I 522 saxatilis Scribn. \& Williams

I523 scabriuscula Williams

I 524 secunda Presl

I525 subaristata Scribn.

I526 Suksdorfii Vasey

I527 sylvestris A. Gray

I528 tenerrima Scribn.

I 529 Tracyi Vasey

I 530 trivialis $\mathrm{L}$.

I53I Tumeri Scribn.

r532 unilateralis Scribn.

I 533 Vaseyana Scribn.

I534 Vaseyochloa Scribn. (P. pulchella Vasey)

I535 Wheeleri Vasey Rydb.
I536 Wolfii Scribn.

\section{Colpodium $3 \% 9$}

I 537 fulvum (Trin.) Griseb.

I 538 mucronata (Hack.) Beal

I539 pendulinum (Laest.) Griseb.

\section{Dupontia 380}

I540 Fisheri R. Br.

r54 I psilosantha Rupr.

\section{Scolochloa 381}

I 542 festucacea (Willd.) Link

\section{Graphephorum $\quad 38 \%$}

I 543 melicoideum (Michx.) Beauv.

I544 muticum (Bolander)

I545 Wolfii Vasey

\section{Panicularia $38: 3$}

(Glyceria)

I546 acutiflora ('Torr.) Kuntze

I 547 Americana ('Torr.) MacM.

$$
\text { (G. grandis Wats.) }
$$

I 548 borealis Nash

I 549 brachyphylla Nash

I $55^{\circ}$ Canadensis (Michx.) Kuntze

I $55^{1}$ elata Nash

I 552 elongata ('Torr.) Kuntze

I 553 fluitans (L.) Kuntze

I 554 laxa Scribn.

I555 nervata (Willd.) Kuntze

I 556 rigida Nash

I 557 obtusa (Muhl.) Kuntze

I $55^{8}$ pallida (Torr.) Kuntze

I559 pauciflora (Presi) Kuntze

\section{Puccinellia $\mathbf{3 8 4}$}

I 560 airoides (Nutt.) Wats \& Coult.

I 56I angustata (R. Br.) Scribn.

(P. maritima var. minor Wats.)

I 562 distans (L.) Parl.

I 563 Lemmoni (Vasey) Scribn.

r 564 maritima (Huds.) Parl.

r 565 simplex Scribn.

Festuca 385

I566 Altaica Trin.

I 567 amethystina I. 

I568 brachyphylla Schultes (F. brevifolia R. Br.)

I 569 Californica Vase7

I 570 campestris Rydberg

I57I capillata Lam.

I 572 confinis Vasey

I 573 dasyclada Hack.

I 574 denticulata Beal

I 575 elatior L.

1576 fratercula Rupr.

I 577 gigantea (L.) Vill.

I 578 Howellii Hack.

I 579 Jonesii Vasey

I 580 microstachya Nutt.

I 58 I ciliata A. Gray

I582 pauciflora Scribn.

I583 Myuros L.

I584 nutans Willd.

I 585 obtusa Spreng.

(F. Shortii Kunth)

I 586 octoflora Walt.

$$
\text { (F. tenella Willd.) }
$$

I 587 ovina L.

I 588 Arizonica (Vasey) Hack.

I 589 duriuscula (L.) Hack.

I 590 polyphylla Vasey

I 59 I pseudovina Hack.

I592 parviflora Ell.

I593 pauciflora Thunb.

I 594 Richardsonii Hook.

I 595 rubra L.

I 596 scabrella Torr.

I 597 sciurea Nutt.

I598 Vaseyana Hack.

I599 versuta Beal

I600 vallicola $R y d b$.

I60I viridula Vasey

\section{Bromus 389}

I602 Aleutensis Trin.

1603 arvensis L.

I604 asper Murr.

I605 breviaristatus (Hook.)Buckley I606 brizaeformis F. \& M.

1607 carinatus H. \& A.

I608 Arizonicus Shear

1609

I6ro
Californicus (Nutt.) Shear densus Shear
I6I I laciniatus Beal

I6I2 linearis Shear

I6I 3 ciliatus L.

I6I 4 Coloradoensis Vasey

I6I5 laeviglumis Scribn.

I6I6 montanus Vasey

I6I 7 pauciflorus Vasey

I6I8 scariosus Scribn.

I6I9 erectus Huds.

I620 Hookerianus Thurb.

I62I hordeaceus L.

I622 glabrescens (Coss.) Shear

I623 intermedius (Guss.) Shear

I624 inermis Leyss.

I625 Kalmii A. Gray

I626 occidentalis Vasey

I627 laevipes Shear

I628 Madritensis L.

I629 marginatus Nees

r63o latior Shear

163I seminudus Shear

I632 maximus Desf.

I633 Gussoni Parl.

I634 Orcuttianus Vasey

r635 grandis Shear

I636 Paciflcus Shear

I637 patulus M. \& K.

I638 polyanthus Scribn.

I639 paniculatus Shear

I640 Porteri (Coult.) Nash

I64I frondosus Shear

I642 Havardii Shear

(B. ciliatus var. minor Munro)

I643 lanatipes Shear

I644 Pumpellianus Scribn.

I645 melicoides Shear

I646 Tweedyi Scribn.

I647 purgans L.

I648 incanus Shear

I649 latiglumis (Scribn.) Shear

I650 Texensis Shear

I65I racemosus L.

I652 commutatus (Schrad.)

I653 Richardsoni Link

r654 pallidus (Hook.) Shear

Hook.

I655 rubens $\mathrm{L}$.

I656 scoparius L. 

I 657 secalinus I.

$165^{8}$ Sitchensis Bong.

r659 squarrosus L.

1660 sterilis L.

I66I subvelutinus Shear

I662 Suksdorfii Vasey

I663 tectorum L.

I664 Trinii Desv.

I665 pallidiflorus Desv.

I666 excelsus Shear

I667 unioloides (Willd.) H. B. K.

I668 Haenkeanus (Presl) Shear

1669 virens Nutt.

r67o vulgaris (Hook.) Shear

167I eximius Shear

1672 robustus Shear

\section{Nardus 394}

I673 stricta L.

\section{Lolium 395}

I674 perenne $\mathrm{L}$.

I675 temulentum L.

\section{Monerma 400}

I676 subulata L.

\section{Lepturus $\quad 40 \%$}

I677 filiformis (Roth) Trin.

I678 incurvatus (L.) Trin.

\section{Scribneria 403}

I679 Bolanderi (Thurb.) Hack.

\section{Agropyron 405}

I680 albicans S. \& S.

I68I Arizonicum S. \& S.

I 682 brevifolium Scribn.

I 683 campestre Goodr. \& Gren.

I684 caninum (L.) R. \& S.

I685 pubescens S. \& S.

I686 dasystachyum (Hook.) Vasey

I687 subvillosum S. \& S.

I688 Elmeri Scribn.

I689 glaucum (Desf.) R. \& S.

r69o Gmelini (Griseb.) S. \& S.

I69I Pringlei S. \& S.

I692 junceum megastachyum Fries

r693 lanceolatum S. \& S.
I694 molle (S. \& S.) Rydb.

(A. spicatum molle S. \& S.)

I695 Parishii S. \& S.

I696 laeve S. \& S.

I697 psendorepens S. \& S.

1698 magnum S. \& S.

1699 repens (L.) Beauv.

I 700 agreste Anders.

I 7OI littoreum Anders.

I7O2 nemorale Anders.

I703 pilosum Scribn.

I704 Richardsonii Schrad.

I705 ciliatum S. \& S.

I706 riparium S. \& S.

I 707 scabrum Beauv.

I708 Scribneri Vasey

I709 Smithii Rydb.

(A. spicatum S. \& S., not Pursh)

r7io Palmeri (S. \& S.) Heller

I I I spicatum (Pursh) Rydb.

(A. divergens Nees)

I7I 2 inerme (S. \& S.) Heller

I 7 3 tenuispicum (S. \& S.)

I 74 strigosum S. \& S. Rydb.

I 7 I 5 tenerum Vasey

I716 ciliatum S. \& S.

I7I7 longifolium S. \& S.

I 7 I 8 tetrastachyum S. \& S.

I7I9 Vaseyi S. \& S.

I 720 violaceum (Hornem.) Vasey

I72I andinum S. \& S.

I722 latiglume S. \& S.

\section{Hordeum 410}

I 723 adscendens H. B. K.

I 724 aegiceras (E. Meyer) Royle

I 725 boreale S. \& S.

I726 Gussoneanum Parl.

I 727 jubatum I.

I728 maritimum With.

I 729 Montanense S. \& S.

I730 murinum L.

I73I nodosum $\mathrm{I}$.

(H. pratense Huds.) depressum S. \& S.

I733 pusillum Nutt. 

Elynums (410)

I 734 ambiguns Vasey \& Scribn.

I 735 angustus Trin.

I 736 arenarius $\mathrm{L}$.

I 737 arenicola S. \& S.

1738 Brownii S. \& S.

I 739 Canadensis L.

I740 capitatus Scribn.

I 74 I ciliatus Scribu.

I 742 condensatus Presl

I 743 dasystachys Trin.

I 744 littoralis (Pall.) Griseb.

I 745 flavescens S. \& S.

1746 glaucus Buckley

(E. Sibiricus var. Americamus)

I747 hirsutiglumis Scribn. (E. intermedius S. \& S.)

r748 innovatus Beal

I749 Macounii Vasey

I750 mollis Trin.

I75I robustus S. \& S.

I $75^{2}$ salinus Jones

I753 Saundersii Vasey

I754 saxicolus S. \& S.

I755 simplex Scribn. \& Williams

I756 luxurians Scribn. \&

Williams

I 757 striatus Willd.

I $75^{8}$ triticoides (Nutt.) Buckley

I759 villosissimus Scribn.

I 760 Virginicus L.

I76I minor Vasey

I 62 submuticus Hook.

\section{Sitanion (410)}

I 763 anomalum J. G. Smith I 764 breviaristatum J. G. Smith

1765 brevifolium J. G. Smith.

- 766 caespitosum J. G. Sinith

I 767 Californicum J. G. Sinith

I768 cinereum J. G. Smith

I 769 elymoides Raf.

(Elymus Sitanion Schultes)

1770 glaber J. G. Sinith

177 I Hanseni (Scribn.) J. (x. Smith

I 772 hystrix (Nutt.) J. C. Smith

I 773 insulare J. G. Smith

I 774 jubatum J. G. Smith
I775 lanceolatum J. G. Smith

I 776 longifolium J. C. Smith

I 777 minus J. G. Smith

I 778 molle J. G. Smith

I779 montanum J. G. Smith

I780 multisetum J. C. Smith

I78 I planifolium J. G. Smith

I 782 polyantherix J. G. Smith

I 783 pubiflorum J. G. Smith

I 784 rigidum J. G. Smith

I 785 strigoswm J. G. Smith

I786 villosum J. Cr. Smith

\section{Hystrix 41\%}

(Asprella)

I 787 Californica (Bolander)

I 788 Hystrix (I.) Millsp.

\section{Arundinaria 414}

I789 macrosperma Michx:

I 790 tecta (Walt.) Muhl.

(A. macrosperma var. suffruticosa Munro)

CYPERACEAE

Lipocarpha $45 \%$

I79I maculata (Michx.) Torr.

\section{Hemicarpha 45:3}

I792 micrantha (Vah1.) Britton

(H. subsquarrosa Nees)

I793 occidentalis A. Gray

\section{Dulichium 458}

I 794 arundinaceum (L.) Britton

(D. spathaceum Pers.)

\section{Websteria (458)}

I795 limnophila S. H. Wright

\section{Cyperus 459}

I796 acuminatus Torr. \& Hook.

I797 amabilis Vahl.

I 798 articulatus $I_{\text {. }}$.

I799 conglomeratus Britton

I 800 bipartitus Torr.

I801 Blodgettii Britton

I 802 brunneus Sw.

I 803 Buckleyi Britton 

I 804 Californicus Wats.

I 805 cephalanthus Torr. \& Hook. I 806 compressus L.

I 807 cylindricus (Ell.) Britton (C. Torreyi Britton).

I 808 cyrtolepis Torr. \& Hook.

I 809 denticarinatus Britton

I 8 IO dentatus Torr.

I 8 I I diandrus Torr.

I8I 2 capitatus Britton

I 8 I 3 dissitiflorus Torr.

I $8 \mathrm{I} 4$ distans $\mathrm{L}$.

I 815 distinctus Steud.

I 8 I 6 echinatus (E11.) Wood

I8I 7 multiflorus (Chapm.)

I 818 Engelmanni Steud.

Britton

I8I9 erythrorhizus Muhl.

1820 pumilus Engelm.

I82I esculentus L.

I822 angustispicatus Britton

I823 Hermanni (Wats.) Britton

I 824 evaginatus Boeckl.

I825 Fendlerianus Boeckl.

I 826 ferox Vahl.

I827 filiculmis Vahl

I 828 filiformis Sw.

I829 flavescens L.

I830 flavicomus Michx.

I 83 I flavomariscus Griseb.

I832 fuligineus Chapm.

I 833 fuscus L.

I834 giganteus Vahl.

1835 Grayi Torr.

I836 Halei Torr.

I837 Hallii Britton

I 838 Haspan L.

I839 Hochstetteri Nees

I840 Houghtoni Torr.

r84 I inflexus Muhl.

$$
\text { (C. aristatus Rottb.) }
$$

I 842 Iria L.

I843 Lancastriensis Porter

I844 laevigatus L.

I845 lateriflorus Torr.

I846 Lecontei Torr.

I847 leucolepis Carey

r848 ligularis L.
I 849 longus L.

I850 Martindalei Britton

I 851 microdontus Torr.

(C. polystachyus var. leptostachyus Boeckl.)

I852 Nuttallii Eddy

I853 ochraceus Vah1.

I854 Olfersianus Kunth.

I855 ovularis (Michx.) Torr.

I856 robustus Boeckl.

I857 sphaericus Boeckl.

I $85^{8}$ oxycarioides Britton

r859 polystachys Rottb.

I 860 Pringlei Britton

I86I pseudovegetus Steud.

$$
\text { (C. calcaratus Nees) }
$$

I 862 reflexus Vahl

I 863 refractus Engelm.

I 864 retrofractus (L.) Torr.

I865 retrorsus Chapm.

I 866 rivularis Kunth

I 867 rotundus L.

I 868 Rusbyi Britton

I869 Schweinitzii Torr.

I870 debilis Britton

I87 I speciosus Vahl

I872 ferruginescens (Boeckl.)

I 873 parvus (Boeckl.) Britton

I874 spectabilis Schreb.

I 875 sphacelatus Rottb.

I 876 stenolepis Torr.

I 877 strigosus L.

I 878 capitatus Boeck1.

I879 compositus Britton

I 880 robustior Kunth

I88I Surinamensis Rottb.

1882 tetragonus Ell.

I 883 uniflorus Torr. \& Hook.

I 884 virens Michx.

I 885 viscosus Ait.

I886 Wrightii Britton

\section{Kyllinga 46\%}

I 887 monocephala L.

I 888 pumila Michx.

I 889 sesquiflora Torr. 



\section{Eriophorum 466}

I 890 alpinum I.

I891 cyperinum L.

I 892 laxum Wats. \& Coult.

I893 gracile Koch

I894 lineatum B. \& H.

I895 pancinervium (Engelm.)

I 896 polystachyon L.

A. A. Eaton

I 897 russeolum Fries

r 898 Schenchzeri Hoppe

I899 vaginatum $\mathrm{L}$.

I900 Virginicum L.

I 901 album A. Gray

\section{Fuirena 46\%}

I 902 scirpoidea Michx.

I 903 simplex Vahl

I 904 squarrosa Michx.

I 905 breviseta Coville

I906 hispida (Ell.) Chapm.

\section{Scirpus 468}

I907 Americanus Pers.

$$
\text { (S. pungens Vahl) }
$$

1908 longispicatus Britton

1909 atratus Fernald

I9I0 atrocinctus Fernald

i9i I brachypodus Fernald

I9I 2 grandis Fernald

I9I3 atrovirens Muhl.

I9I4 pallidus Britton

I9I 5 caespitosus L.

I9I6 Californicus (C. A. Meyer)

Britton

\section{7 eampestris Britton}

I9I8 carinatus A. Gray

I9I9 cernutus Vahl

I920 Clintoni A. Gray

I92I criniger A. Gray

1922 Cubensis Poepp. \& Kunth

I 923 cylindricus (Torr.) Britton

$$
\text { (S. Canbyi A. Gray) }
$$

I 924 cyperinus (I.) Kuntl

1925 Andrewsii Fernald

i 926 condensatus Fernald

I927 debilis Pursh

1928 divaricatus Fill.
I929 Eriophorum Michx.

I930 fluviatilis ('Torr.) A. Gray

I 931 Georgianus Harper

I932 Hallii A. Gray

1933 heterocarpus Wats.

1934 lacustris L.

I935 occidentalis Wats.

I936 lineatus Michx.

I937 microcarpus Presl

(S. sylzaticus var. digynus)

1938 mucronatus L.

I939 nanus Spreng.

(Eleocharis pygmaea Torr)

I940 anachaetus Britton

I94I Nevadensis Wats.

I 942 Novae-Angliae Britton

I 943 Olneyi A. Gray

I 944 paludosus Aven Nelson

I 945 pauciflorus Lightf. (Elencharis pauciflorus Link)

I 946 Peckii Britton

I947 pedicellatus Fernald

I948 pullus Fernald

I949 planifolius Muh1.

I950 polyphyllus Vah1.

I95I pumilus Vah1.

I952 robustus Pursh

I 953 rubrotinctus Fernald

I 954 confertus Fernald

I 955 rufus (Huds.) Schrad.

1956 Smithii A. Gray

I 957 subterminalis Torr

I $95^{8}$ sylvaticus $\mathrm{L}$.

I 959 Bissellii Fernald

I960 Torreyi Olney

\section{Eleocharis 469}

I 96I acicularis (L.) R. \& S.

I962 acuminata (Mul11.) Nees

(E. compressa Sull.)

I 963 albida Torr.

I 964 Berlandieri Britton

I 965 atropurpurea (Retz.) Kunth

I 966 Baldwinii Chapm.

I 967 Bolanderi A. Gray

I 968 bicolor Chapm.

I969 capillacea Kunth

I970 capitata (I.) R. Br. 

I97 I cancellata Wats.

I 972 cellulosa Torr.

I 973 cylindrica Buckley

I974 diandra C. Wright

I975 depressa Fernald

I976 Dombeyana Kunth

1977 elongata Chapm.

I 978 Engelmanni Stend.

I 979 detonsa A. Gray

I 980 robusta Fernald

I 98 I glaucescens (Willd.) Schultes

I982 intermedia (Muhl.) Schultes

I 983 interstincta (Vahl) R. \& S. (E. equisetoides Torr.)

I 984 lanceolata Fernald

I985 Macounii Fernald

I 986 melanocarpa Torr.

1987 microcarpa Torr.

(E. Torreyana Boeckl.)

I 988 monticola Fernald

I 889 leviseta Fernald

I 990 mutata (L.) R. \& S.

(E. quadrangulata $\mathrm{R}$. Br.)

I99I nodulosa Schultes

I 992 ochreata (Nees) Steud.

I 993 olivacea Torr.

I 994 obtusa Schultes

I995 gigantea Fernald

I996 jejuna Fernald

I997 ovata (Roth) R. \& S.

I 998 Heuseri Uechtritz.

I999 palustris (L.) R. \& S.

2000 glaucescens (Willd.)

200I Watsoni (Bab.) Clarke

2002 Parishii Britton

2003 prolifera Torr.

2004 Robbinsii Oakes

2005 rostellata Torr.

2006 occidentalis Wats.

2007 tenuis (Willd.) Schultes

2008 thermalis Rydb.

2009 tortilis (Link) Schultes

2010 tricostata Torr.

20 I tuberculosa (Michx.) R. \& S.

2012 Wolfii A. Gray

\section{Fimbristylis 471}

2013 apus Wats.
2014 autumnalis (I.) R. \& S.

2015 castanea (Michx.) Vahl.

2016 ciliatifolia Britton

2017 laxa Vahl.

2018 miliacea L.

2019 monostachya Britton

2020 puberula (Michx.) Vahl

202 I schoenoides Vahl

2022 spadicea $\left(I_{\text {. }}\right)$ Vahl

2023 thermalis Wats.

2024 Vahlii (Lam.) I,ink

Stenophyllus (471)

(Fimbristylis in part)

2025 capillaris (L.) Britton

2026 coarctatus (E11.) Britton

2027 ciliatifolius (E11.) Mohr

2028 Floridanus Britton

2029 Funckii (Steud.) Britton

2030 Stenophyllus (Ell.) Britton

203 I Warei (Torr.) Britton

\section{Psilocarya 47\%}

2032 nitens (Vahl) Wood

2033 scirpoides Torr.

2034 Texensis Torr. \& Hook.

\section{Dichromena 473}

2035 colorata (L.) A. S. Hitchc.

(D. leucocephala Michx.)

2036 diphylla Torr.

2037 latifolia Baldwin

\section{Cladium 489}

2038 effusum Torr.

2039 mariscoides (Muhl.) Torr.

2040 Mariscus Californicus Wats.

Rhynchospora $49 \%$

204I alba (L.) Vahl

2042 macra Clarke

2043 axillaris (Lam.) Britton

(R. cephalantha A. Gray)

2044 microcephala Britton

2045 Baldwinii A. Gray

2046 brachychaeta Sauv.

2047 caduca Ell.

2048 capillacea Torr.

2049 laeviseta E. J. Hill 

2050 Chapmani M. A. Curtis

205I ciliata Vahl

2052 compressa Carey

2053 corniculata (Lam.) A. Gray

2054 macrostachya ('Torr.) Britton

2055 corymbiformis (Benth.)Britton 2056 cymosa Ell.

2057 globularis Chapm.

$205^{8}$ decurrens Chapm.

2059 distans (Michx.) Britton

2060 tenuis (Baldwin) Britton

206I divergens Chapm.

2062 dodecandra Baldwin

2063 fascicularis Nutt.

2064 trichodes Chapm.

2065 filifolia A. Gray

2066 fusca (L.) R. \& S.

2067 fuscoides Clarke

2068 glomerata (L.) Vahl

2069 discutiens Clarke

2070 leptocarpa Chapm.

$207 \mathrm{I}$ minor Britton

2072 paniculata(A.Gray)Chapm.

2073 gracilenta A. Gray

2074 Grayi Kunth

2075 Harveyi Boott

2076 inexpansa (Michx.) Vahl

2077 jubata Liebm.

2078 Knieskernii Carey

2079 microcarpa Baldwin

2080 miliacea A. Gray

208I oligantha A. Gray

2082 pallida M. A. Curtis

2083 patula A. Gray

2084 plumosa Eill.

2085 punctata Ell.

2086 pusilla Chapm.

2087 rariflora Eill.

2088 schoenoides (E11.) Britton

2089 semiplumosa A. Gray

2090 scutellata Griseb.

209I stenophylla Chapm.

2092 Torreyana A. Gray

2093 Tracyi Britton

\section{Scleria $\mathbf{5 1 5}$}

2094 Baldwinii Stend.
2095 costata Britton

2096 ciliata Michx.

2097 gracilis Ell.

2098 hirtella Sw.

2099 lithosperma filiformis Britton

2 I00 oligantha Michx.

2IOI pauciflora Muhl.

$2 \mathrm{IO2}$ Caroliniana Wood

$2 \mathrm{IO} 3$ Elliottii Wood

2104 elongata Britton

2105 glabra Chapm.

2 I06 reticularis Michx.

2 ro7 obscura Britton

2108 pubescens Britton

2I09 Torreyana Walp.

2IIO triglomerata Michx.

2III minor Britton

2 II2 verticillata Muh1.

\section{Elnya 5\%0}

2 I 3 Bellardi (Al1.) C. Koch

\section{Kobresia 5*2}

2II4 bipartita (A11.) Britton

2II5 brevifolia Rottb.

2I 6 caespitosa Nees

2 II 7 odorata Vahl

2 I 8 pumila Michx.

\section{Uncinia $5 \% 4$}

2 I 9 microglochin (Wahl.) Spreng.

\section{Carex 5\%5}

2 I 20 abacta Bailey

(C. Michauxiana Boott)

2I 2 a abreviatta Prescott

(C. Torreyi Tuckerman)

2I 22 ablata Bailey

2 I 23 acutiformis Ehrh.

2I 24 acutina Bailey

2125 adusta Boott

2I 26 aestivalis M. A. Curtis

2I27 Alaskana Boeckl.

2128 alata Torr.

2r 29 albida Bailey

2 I 30 albolutescens Schwein.

2I 3I Albursina Sheldon

2r 32 alma Bailey

2 I33 alopecoidea Tuckerman

2.I 34 sparsispicata Dewey 

21 35 alpina Sw.

2136 altocaulis (Dewey) Britton

(C. Saltuensis Bailey)

2I37 ambusta Boott

21 38 amphibola Steud.

(C. grisea var. rigida Bailey)

2139 globosa Bailey

2I 40 amplifolia Boott

2I I I androgyna Bailey

2142 aquatilis Wahl.

2 I 43 arcta Boott

(C. canescens var. polystachya)

2144 arctata Boott

2 I 45 Faxoni Bailey

2146 arenaria $\mathrm{L}$.

2147 Arkansana Bailey

2148 aristata $\mathrm{R}$. Br.

2I 49 Asa-Grayi Bailey

(C. Grayi Carey)

2I 50 Assiniboinensis W. Boott

2 I 5 athrostachya Olney

2I 52 Atlantica Bailey

2 I 53 atrata $\mathbf{L}$.

2I 54 atratiformis Britton

2I 55 aurea Nutt.

2I 56 celsa Bailey

2I 57 auriculata Bailey

2I $5^{8}$ Austro-Caroliniana Bailey

2I59 Baileyi Britton

2I60 Baltzellii Chapm.

216r Barbarae Dewey

2162 bella Bailey.

2163 Bicknellii Britton

2164 bicolor All.

2 I65 bifida Boott

2166 Bigelovii Torr.

(C. vulgaris var. hyperborea)

2r67 Blankinshipii Fernald

2I68 Breweri Boott

2169 Brittoniana Bailey

2170 bromoides Schk.

217 I brunnescens (Pers.) Poir.

(C. canescens var. alpicola)

2 I 72 gracilior Britton

2 I 73 bullata Schk.

2174 Californica Bailey

2175 canescens $\mathrm{L}$.

2176 brunnea Macoun
2I77 dubia Bailey

2178 Oregana Bailey

2179 robustina Macoun

2180 capillaris L.

2I8I capitata L.

2182 Careyana Torr.

2 I 83 Caroliniana Schwein.

21 84 castanea Wahl.

2 I 85 cephaloidea Boott

2i 86 cephalophora Muhl.

2187 Chapmani Sartw.

21 88 Cherokeensis Schw.

2189 chordorhiza L. f.

2190 cinnamomea Olney

219I circinata Meyer

2 I92 Collinsii Nutt.

(C. subulata Michx.)

2193 comosa Boott

2I94 concinna R. Br.

2 I95 Congdoni Bailey

2196 conjuncta Boott

2197 conoidea Schk.

2 I98 costellata Britton

2199 Crawei Dewey

2200 crinita Lam.

2201 minor Boott

$22 \mathrm{O} 2$ cristatella Britton

2203 Crus-Corvi Shuttlw.

2204 cryptocarpa C. A. Meyer

2205 variegata (Drej.) Britton

2206 cuspidata Wahl.

2207 Davisii Schwein. \& Torr.

2208 debilis Michx.

2209 decidua Boott

22 IO decomposita Muhl.

22 II deflexa Hornem.

22 2 Deanei Bailey

$22 \mathrm{I} 3$

$22 \mathrm{I} 4$

22 I 5 densa Bailey

Farwellii Britton

Rossii Bailey

2216 Deweyana Schwein.

22 I 7 Bolanderi W. Boott

22 I 8 sparsiflora (Olney) Bailey

2219 digitalis Willd.

2220 copulata Bailey

222 I distans L.

2222 Douglasii Boott

2223 Williamsii Rydb. 

2224 durifolia Bailey

$$
\text { (C. Backii Boott) }
$$

2225 Eggertii Bailey

2226 Eleocharis Bailey

2227 Elliottii Schwein. \& Torr.

2228 Engelmanni Bailey

2229 exilis Dewey

2230 exsiccata Bailey

223 I globosa (Olney) Bailey

2232 pungens Bailey

2233 extensa Gooden.

2234 festiva Dewey

2235 Haydeniana Boott

2236 minor Olney

2237 pachystachy (Cham.)

2238 stricta Bailey

2239 viridis Bailey

2240 festucacea Willd.

224 I feta Bailey

2242 multa Bailey

2243 filifolia Nutt.

2244 filiformis L.

2245 flacca Schreb.

2246 flaccosperma Dewey

2247 flava I.

2248 graminis Bailey

2249 recterostrata Bailey

2250 Floridana Schwein.

225I foenea Willd.

2252 perplexa Bailey

2253 folliculata L.

2254 australis Bailey

2255 formosa Dewey

2256 Frankii Kunth (C. stenolepis Torr.)

2257 Franklinii Boott

2258 Fraseri Andr.

2259 fulva Gooden.

2260 fusca All.

226I Gayana Desv.

2262 Geyeri Boott

2263 glareosa Wahl.

2264 ursina (Dewey) Bailey

2265 glauca Scop.

2266 glaucodea Tuckerman

2267 globosa Boott

2268 Gmelini Hook.
2269 Goodenovii J. Gay

(C. vulgaris Fries)

2270 gracillima Schwein.

227 I grandis Bailey

2272 Helleri Bailey

2273 granularis Muhl.

2274 Schriveri Britton

2275 gravida Bailey

2276 laxifolia Bailey

2277 grisea Wahl.

2278 gynandra Schwein.

2279 Porteri (Olney) Britton

228 o gynodynama Olney

228I Halleriana Asso

2282 Hallii Olney

Bailey

2283 Hartii Dewey

2284 Hassei Bailey

2285 Haydeni Dewey

(C. stricta var. decora Bailey)

2286 Heleonastes Ehrh.

2287 Hendersoni Bailey

2288 herbariorum Bailey

2289 heteroneura W. Boott

2290 Hitchcockiana Dewey

229 I hirta L.

2292 Hoodii Boott

2293 nervosa Bailey

2294 Hookeriana Dewey

2295 Houghtonii Torr.

2296 Howellii Bailey

2297 hystricina Muhl.

2298 angustior Bailey

2299 Dudleyi Bailey.

2300 Idahoa Bailey

2301 illota Bailey

2302 incurva Lightf.

2303 inops Bailey

2304 interior Bailey

2305 capillacea Bailey

2306 interrupta Boeckl.

2307 impressa Bailey

2308 intumescens Rudge

2309 Fernaldii Bailey

23IO invisa Bailey

23II irrasa Bailey

2312 Jamesii Schwein.

23I3 Jonesii Bailey 

2314 Joori Bailey

23 I 5 juncella Fries

$$
\text { (C. Kelloggii Boott) }
$$

2316 juncea Willd.

23 I 7 Krausei Boeckl.

23I 8 laciniata Boott

2319 laeviconica Dewey

2320 lagopina Wahl.

232 I lanuginosa Michx.

(C. filiformis var. latifolia)

2322 Kansana Britton

2323 laxiculmis Schwein.

2324 laxiflora Lam.

2325 blanda (Dewey) Boott

2326 divaricata Bailey

2327 Michanxii Bailey

2328 patulifolia (Dewey) Carey

2329 varians Bailey

2330 Leavenworthii Dewey

233 I leiocarpa Meyer

2332 leiorhyncha Meyer

2333 Lemmoni W. Boott

2334 lenticularis Michx.

2335 leporina L.

2336 leptalea Wahl.

(C. polytrichoides Muh1.)

2337 Liddoni Boott

2338 limosa L.

2339 stygia (Fries) Bailey

2340 littoralis Schwein.

234 I livida (Wahl.) Willd.

2342 longirostris Torr.

2343 Louisianica Bailey

2344 lupuliformis Sartwell

(C. lupulina var. polystachya)

2345 lupulina Muhl.

2346 Bella-villa (Dewey) Bailey

2347 lurida Wahl.

2348 exudans Bailey

2349 flaccida Bailey

2350 parvula (Paine) Bailey

235 I luzulaefolia W. Boott

2352 luzulina Olney

2353 Lyoni Boott

2354 macrocephala Willd.

2355 macrochảeta C. A. Meyer

2356 macrokolea Stend.

2357 Magellanica I am.
$235^{8}$ marcida Boott

2359 alterna Bailey

2360 maritima Mueller

2361 Meadii Dewey

2362 melanocarpa Chapm.

2363 membranopacta Bailey

2364 Mendocinensis Olney

2365 Mertensii Prescott

2366 microdonta Torr.

2367 miliaris Michx.

2368 major Bailey

2369 misandra R. Br.

2370 miser Buckley

237 I monile Tuckerman

2372 colorata Bailey

2373 monstrosa Bailey

2374 obtusisquamis Bailey

2375 Pacifica Bailey

3376 Montanensis Bailey

2377 Muhlenbergii Schk.

2378 australis Olney

2379 Xalapensis (Kunth)Britton

2380 multicaulis Bailey

238 I multimoda Bailey

2382 muricata L.

$23^{8} 3$ Muskingumensis Schwein.

2384 nardina Fries

2385 Nebraskensis Dewey

2386 praevia Bailey

2387 ultriformis Bailey

2388 nervina Bailey

2389 nigricans C. A. Meyer

2390 nigro-marginata Schwein.

2391 Norvegica Willd.

2392 nova Bailey

2393 Nova-Angliae Schwein.

2394 nudata W. Boott

2395 obessa minor Boott

2396 oblata Bailey

2397 luzuliformis Bailey

2398 oblita Steud.

(C. venusta var. minor Boeck1.)

2399 obnupta Bailey

2400 obtusata Lilj.

24 or occidentalis Bailey

2402 Oederi Retz.

2403 oligocarpa Schk.

2404 oligosperma Michx. 

2405 Oregonensis Olney

2406 ovalis Gooden.

2407 oxylepis Torr. \& Hook.

2408 pallescens $L$.

2409 panicea L.

24 IO paniculata L.

24 II pansa Bailey

24I 2 Parryana Dewey

24I3 paucifiora Lightf.

2414 pedicellata (Dewey) Britton

\section{(C. communis Bailey)}

24 I 5 Wheeleri (Bailey) Britton 2416 pedunculata Muhl.

24I7 Pennsylvanica Lam.

2418 vespertina Bailey

24 I9 petricosa Dewey

2420 phyllomanica W. Boott

242 I physocarpa Presl

2422 picta Steud.

2423 plantaginea Lam.

2424 platyphylla Carey

2425 podocarpa $\mathrm{R}$. Br.

2426 polymorpha Muhl.

2427 praecox Jacq.

2428 praegracilis W. Boott.

2429 prasina Wahl.

2430 praticola Rydb.

\section{(C. pratensis Drej.)}

243I furva (Bailey) Heller

2432 Preslii Steud.

2433 pseudocyperus L.

2434 pseudoscirpoidea Rydb.

2435 ptychocarpa Stend.

2436 pubescens Muhl.

2437 Pyrenaica Wahl.

2438 quadrifida Bailey

2439 caeca Bailey

2440 lenis Bailey

244I Qualicumensis Bailey

2442 Raeana Boott

(C. mitiaris var. aurea Bailey)

2443 rariflora J. E. Smith

2444 Raynoldsii Dewey

2445 Redowskyana C. A. Meyer

(C. gynocrates Wormsk.)

2446 remota L.

2447 retroflexa Muhl.

2448 retrorsa Schwein.
2449 Richardsoni R. Br.

2450 rigida Gooden.

245 I riparia W. Curtis

2452 rosea Schk.

2453 radiata Dewey

2454 rupestris All.

2455 salina Wahl.

2456 robusta Bailey

2457 Sartwellii Dewey

$245^{8}$ saxatilis L.

2459 Grahami H. \& A.

2460 scaberrima Scheele

246 r scabrata Schwein.

2462 Schweinitzii Dewey

2463 scirpoidea Michx.

2464 scoparia Schk.

2465 minor W. Boott

2466 senta Boott

2467 serratodens W. Boott

2468 setacea Dewey

2469 setifolia (Dewey) Britton

(C. eburnea Boott)

2470 Shortiana Dewey

247 I siccata Dewey

2472 silicea Olney

2473 Sitchensis Prescott

2474 sparganioides Muhl.

2475 specifica Bailey

2476 spissa Bailey

2477 spreta Bailey

2478 squarrosa L.

2479 stenophylla Wahl.

2480 sterilis Willd.

248 I angustata (Carey) Bailey

2482 cephalantha Bailey

2483 excelsior Bailey

2484 straminea Willd.

2485 aperta Boott

2486 australis Bailey

2487 ferruginea Bailey

2488 hyalina Boott

2489 mixta Bailey

2490 mirabilis (Dewey) Tuck.

249I reniformis Bailey

2492 stipata Muhl.

2493 straminiformis Bailey

2494 stricta Lam.

2495 angustata (Boott) Bailey 

2496

2497

2498 styloflexa Buckley

2499 stylosa C. A. Meyer

2500 subfusca W. Boott

250 or subspathacea Wormsk.

2502 supina Willd.

2503 sychnocephala Carey

2504 tenella Schk.

2305 tenera Dewey

2506 invisa (W. Boott) Britton

2507 tenuiflora Wahl.

2508 tenuirostris Olney

2509 tenuis Rudge

(C. debilis var. Rudgei Bailey)

2510 erectior Britton

25 I I interjecta (Bailey) Britton

25I2 teretiuscula. Gooden.

2513 ampla Bailey

2514 prairea (Dewey) Britton

2515 tetanica Schk.

2516 Canbyi Porter

25I7 Woodii (Dewey) Bailey

2518 Texensis (Torr.) Bailey

2519 Tolmiei Boott.

2520 angusta Bailey

2521 nigella (Boott) Bailey

2522 subsessilis Bailey

2523 torta Boott

2524 triangularis Boeckl.

2525 tribuloides Wahl.

2526 Bebbii Bailey

2527 moniliformis ('Tuckerman)

2528 triceps Michx.

2529 trichocarpa Muhl.

2530 triquetra Boott

253 I trisperma Dewey

2532 Tuckermani Dewey

2533 turgescens Torr

2534 turgidula Bailey

2535 typhinoides Schwein.

2536 ultra Bailey

2537 umbellata Schk.

$253^{8}$ vicina Dewey

2539 usta Bailey

2540 utriculata Boott
2541 minor Boott

2542 varia Muhl.

2543 australis Bailey

2544 variabilis Bailey

2545 altior Rydb.

2546 venusta 'Dewey

2547 vernacula Bailey

2548 vestita Willd.

2549 vicaria Bailey

2550 virescens Muhl.

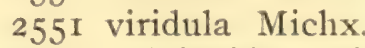

$255^{2}$ vulpinoidea Michx.

2553 Walteriana Bailey (C. striata Michx.)

2554 Watsoni Olney

2555 Whitneyi Olney

2556 Willdenovii Schk.

2557 xanthocarpa Bicknell

$255^{8}$ annectens Bicknell

2559 xerantica Bailey.

2560 Yosemitana Bailey

PALMACEAE

Rhaphidophyllum $\mathbf{5 3 1}$

256r hystrix Wendl. \& Drude

\section{Thrinax 536}

2562 Floridana Sargent

(T. parviflora of Chapman's Flora)

2563 Keyensis Sargent

2564 microcarpa Sargent

\section{Coccothrinax 536a}

2565 Garberi (Chapm.) Sargent

(Thrinax Garberi Chapm.)

2566 jucunda Sargent

(Thrinax argentea of Chapman's Flora)

Neowashingtonia $\mathbf{5 4} 3$

(Washingtonia)

2567 filamentosa (Wendl.) Sudw.

( $W$. filifera Wendl.)

2568 robusta (Wend1.) Heller

\section{Sabal 547}

2569 Adansonii Guerns.

2570 megacarpa Chapm. 

257 I Eatonia Swingle

2572 Mexicana Mart.

2573 Palmetto (Walt.) R. \& S.

Serenoa $\mathbf{5 4 8}$

2574 arborescens Sargent

2575 serrulata B. \& H.

Pseudophoenix $\mathbf{5 9 9}$

2576 Sargentii Wendl.

\section{Oreodoxa 613}

2577 regia H. B. K.

\section{Cocos 663}

2578 nucifera L.

\section{ARACEAE}

\section{Acorus 694}

2579 Calamus L.

\section{Lysichiton $70 \%$}

2580 Kamtschatcensis Schott

\section{Spathyema $\% 08$}

(Symplocarpus)

258 I foetida (L.) Raf.

\section{Orontium 709}

2582 aquaticum L.

\section{Calla 710}

$25^{8} 3$ palustris L.

\section{Peltandra 74}

2584 sagittaefolia (Michx.) Morong (P. alba Raf.)

2585 Virginica (L.) Kunth

(P. undulata Raf.)

Xanthosma $\mathbf{7 5 8}$

2586 sagittifolium Schott

Arisaema 786

2587 Dracontium (L.) Schott

2588 polymorphum Chapm.

2589 quinatum Chapm.

2590 triphyllum (L.) Torr.

259 I pumila Peck.

\section{Pistia $\% 91$}

2592 stratiotes spathulata Engelm.
LEMNACEAE

Spirodela $\mathbf{7 9 4}$

2593 polyrhiza (L.) Schleid

\section{Lemna $\mathbf{9 9 5}$}

2594 cyclostasa (E11.) Chev.

2595 gibba L.

2596 minima Philippi

2597 minor L.

2598 perpusilla Torr.

2599 trinervis Austin

2600 trisulca L.

\section{Wolffia 1996}

26or Columbiana Karst.

2602 papulifera Thompson

2603 punctata Griseb.

\section{Wolffiella (196)}

2604 Floridana (J. D. Smith)

Thompson

2605 lingulata Hegelm.

2606 oblonga (Philippi) Hegelm.

\section{MAYACACEAE}

\section{Mayaca $\mathbf{8 2 5}$}

2607 Aubleti Michx.

(M. Michauxii Schott \& Endl.)

\section{XYRIDACEAE}

\section{Xyris $8 \% 6$}

2608 ambigua Beyrich

2609 Baldwiniana R. \& S.

26Io brevifolia Michx.

26I I Caroliniana Walt.

26I 2 communis Kunth

26r3 Elliottii Chapm.

26I 4 fimbriata Eill.

26 I 5 flabelliformis Chapm.

26r6 flexuosa Muhl.

2617 iridifolia Chapm.

26I8 montana Ries

26r9 neglecta Small

2620 platylepis Chapm.

262 I serotina Chapm.

2622 Smalliana Nash

2623 setacea Chapm. 

2624 stricta Chapm.

2625 tenuifolia Chapm.

2626 torta J. E. Smith

\section{ERIOCAULACEAE}

\section{Eriocaulon 828}

2627 compressum Lam. (E. gnaphalodes Michx.) 2628 decangulare L. 2629 Ravenelii Chapm. 2630 septangulare With. 263I Texense Kornicke

\section{Dupatya 830} (Paeplanthus) 2632 flavidula (Michx.) Kuntze.

\section{Lachnocaulon $\mathbf{8 3 1}$} 2633 anceps (Walt.) Morong (E. Michauxii Kunth) 2634 glabrum Kornicke

\section{BROMELIACEAE}

\section{Hechtia 886}

2635 Texensis Wats.

\section{Tillandsia $\mathbf{8 9 0}$}

2636 Balbisiana R. \& S.

2637 Bartramii Ell.

2638 bulbosa Hook.

2639 caespitosa LeConte

2640 fasciculata Sw.

2641 flexuosa Sw.

2642 Howzeavi Morren

2643 juncea L,eConte

2644 recurvata L

2645 setacea Sw.

2646 usneoides L.

2647 utriculata LeConte

2648 Valenzuelana A. Rich.

2649 Wilsoni Wats.

Catopsis $\mathbf{8 9 2}$

2650 nitida Baker

COMMELINACEAE

Commelina 896

265 communis $\mathrm{L}$.
2652 crispa Wooton

2653 dianthifolia DC.

2654 elegans H. B. K.

2655 erecta $I_{1}$.

2656 hirtella Vahl.

2657 nudiflora L.

$265^{8}$ Swingleana Nash

2659 Virginica L.

\section{Tinantia 910}

2660 anomala ('Torr.) Clarke

\section{Tradescantia 911}

266r bracteata Small 2662 brevicaulis Raf.

2663 comata Small

2664 foliosa Small

2665 gigantea Rose

2666 Floridana Wats.

2667 hirsuticaulis Snall

2668 humilis Rose

2669 longifolia Small

2670 micrantha Torr.

267 I montana Shuttlw.

2672 occidentalis Britton

2673 pilosa J. G. C. Lehm.

2674 pinetorum Greene

2675 reflexa Raf.

2676 rosea Vent.

2677 scopulorum Rose

2678 Vitginiana L.

2679 flexuosa Wats.

Treleasea $911 \mathrm{a}$

2680 brevifolia (Torr.) Rose

(Tradescantia brevifolia Torr.)

268I leiandra (Torr.) Rose

(Tradescantia leiandra Torr.)

\section{PONTEDERIACEAE}

\section{Piaropus 921}

(Eichhornia)

2682 crassipes (Mart.) Britton

\section{Pontederia 9\%'}

2683 cordata L.

2684 lancifolia (Muhl.) Morong 

Heteranthera $9 \% 4$

2685 dubia (Jacq.) MacM. (H. graminea Vahl.)

2686 limosa (Sw.) Willd. 2687 Mexicana Wats. 2688 reniformis R. \& P.

\section{JUNCACEAE}

\section{Juncus 936}

2689 acuminatus Michx. 2690 debilis (A. Gray) Engelm. 269 I acutus L.

2692 articulatus L.

2693 obtusatus Engelm.

2694 Balticus Willd.

2695 littoralis Engelm.

2696 montanus Engelm.

2697 Watsoni Coville

2698 biglumis L.

2699 Bolanderi Engelm.

2700 brachycarpus Engelm.

2701 brachycephalus (Engelm.)

Buch.

2702 bufonius L.
2703 bulbosus L.
2704 Caesariensis

(J. asper Engelm.)

2705 Canadensis J. Gay

2706 brevicaudatus Engelm.

2707 Kuntzei Buch.

2708 subcaudatus Engelm.

2709 castaneus Smith

27 IO chlorocephalus Engelm.

27 II confusus Coville

$27 \mathrm{I} 2$ conglomeratus L.

27I3 Cooperi Engelm.

2714 dichotomus E11.

27 I 5 diffusissimus Buckley

2716 dubius Engelm.

2717 effusus L.

2718 Elliottii Chapm.

27 I9 ensifolius Wikstr.

2720 falcatus E. Meyer

$272 \mathrm{I}$ Alaskensis Coville

2722 filiformis L.

2723 filipendulus Buckley
2724 Georgianus Coville

2725 Gerardi Lois

2726 Greenei Oakes \& Tuckerman

2727 gymnocarpus Coville

(J. Simithii Engelm.)

2728 Haenkei F. Meyer

2729 Hallii Engelm.

2730 Kelloggii Fngelm.

273I Lescurii Bolander

2732 longistylis Torr.

2733 macrophyllus Coville

2734 marginatus Rostk.

2735 aristulatus (Michx.) Coville

2736 pinetorum Coville

2737 setosus Coville

2738 maritimus Lam.

2739 megacephalus M. A. Curtis

(J. scirpoides var. echinatus

2740 Mertensianus Bong.

Fingelm)

274I Mexicanus Willd.

2742 militaris Bigel.

2743 Nevadensis Wats.

2744 nodosus L.

2745 Texanus Engelm.

2746 obtusatus Engelm.

2747 Oreganus Wats.

2748 orthophyllus Coville

2749 oxymeris Engelm.

2750 Parryi Engelm.

$275^{I}$ patens E. Meyer

$275^{2}$ paucicapitatus Buch.

2753 pelocarpus E. Meyer

2754 abortivus (Chapm.) Coville

2755 subtilis (E. Meyer)Engelm.

2756 phaeocephalus Engelm.

2757 polycephalus Michx.

$275^{8}$ Rugelii Buch.

2759 repens Michx.

(Cephaloxys fabellata Desv.)

2760 Richardsonianus Schult.

(J. alpinus var. insignis Fries)

276I robustus (Engelm.) Coville

2762 Roemerianus Scheele

2763 rugulosus Engelm.

2764 saginoides Engelm.

2765 scirpoides Lam.

2766 secundus Beauv. 

2767 setaceus Rostk.

2768 stygius L.

2769 subtriflorus (F. Meyer) Coville

2770 Suksdorfii Rydb.

2771 supiniformis Fingelm.

2772 tenuis Willd.

2773 congestus Engelm.

2774 occidentalis Coville

2775 Torreyi Coville

(J. nodosus var. megacephalus

$$
\text { Torr.) }
$$

2776 trifidus L.

2777 triformis Engelm.

2778 brachystylus Engelm.

2779 triglumis L.

2780 trigonocarpus Steud.

278 I truncatus Coville

2782 Tweedyi Rydb.

2783 validus Coville

2784 Vaseyi Engelm.

2785 xiphioides E. Meyer

2786 auratus Engelm.

2787 macranthus Engelm.

2788 montanus Engelm.

\section{Juncoides 93 '}

(Luzula)

2789 arcuatum (Wahl.) Kuntze

2790 campestre (L.) Kuntze

2791 multiflorum (Fhrh.)

Sheldon

2792 sudeticum (Willd.) Coville 2793 comosum (E. Meyer) Sheldon 2794 congestum (Thuill.)

Sheldon

2795 macranthum (Wats) Parish 2796 subsessile (Wats.) Sheldon

2797 divaricatum (Wats.) Coville 2798 glabratum (Hoppe) Sheldon 2799 hyperboreum (R. Br.) Sheldon 2800 nemorosum (Poll.) Kuntze 280 I nivale (Laest.) Coville 2802 parviflorum (Ehrh.) Coville

(L. spadicea var. melanocarpa

2803 pilosum (L.) Kuntze (L. vernalis DC.)

2804 spicatum (L.) Kuntze
STEMONACEAE

\section{Croomia 940}

2805 pauciflora Torr.

\section{LILIACEAE}

\section{Tofieldia 942}

2806 coccinea Richards

2807 major Hook.

2808 glabra Nutt.

2809 glutinosa (Michx.) Pers.

28Io occidentalis Wats.

28II palustris Huds.

$28 \mathrm{I} 2$ racemosa (Walt.) B. S. P.

(T.pubens Ait.)

\section{Pleea 943}

28I 3 tenuifolia Michx.

\section{Abama 944}

(Narthecium)

28I4 Americana (Ker) Morong 28I5 Californica (Baker) Heller

\section{Xerophyllum 948}

28I6 asphodeloides (I.) Nutt.

(X. setifolium Michx.)

28I 7 Douglasii Wats.

28I8 tenax Nutt.

\section{Helonias 949}

28 I9 bullata L.

\section{Chamaelirium 950}

2820 luteum (I.) A. Gray

(C. Carolinianum Willd.)

\section{Chrosperma 955}

(Amianthemum)

282 I muscaetoxicum(Walt.)Kuntze

\section{Stenanthium $95 \%$}

2822 gramineum (Ker) Morong

$$
\text { (S. angustifolium Kunth) }
$$

2823 occidentale A. Gray

2824 robustum Wats.

\section{Zygadenus $\mathbf{9 5 8}$}

2825 angustifolius Wats.

2826 elegans Pursh 

2827 Fremontii Torr.

2828 glaberrimus Michx.

2829 leimanthoides (A. Gray) Wats.

2830 Nuttallii (A. Gray) Wats.

283I paniculatus (Nutt.) Wats.

2832 porrifolius Greene

2833 venenosus Wats.

\section{Melanthium 959}

2834 dispersum Small

2835 latifolium Desr.

2836 longipedicellatumA.Brown 2837 Virginicum L.

\section{Veratrum 960}

2838 Californicum Durand

2839 caudatum Heller

2840 fimbriatum A. Gray

284I intermedium Chapm.

2842 parviflorum Michx.

(Melanthium parviflorum Wats.) 2843 viride Ait.

2844 Woodii Robbins

\section{Uvularia 966}

2845 grandiflora J. E. Smith

2846 perfoliata L.

2847 puberula Michx.

(Oakesia puberula Wats.)

2848 sessilifolia L.

(Oakesia sessilifolia Wats.)

2849 . nitida (Britton) Morong

\section{Anthericum $\mathbf{9 8 9}$}

2850 serotinum Baker

285I Torreyi Baker

\section{Eremocrinum 991}

2852 albomarginatum Jones

\section{Oxytria 1006}

(Schoenolivion)

2853 albiflora (Raf.) Pollard

2854 crocea (Michx.) Raf.

2855 Texana (Scheele) Pollard

\section{Hastingsia (1006)}

2856 alba (Durand) Wats.

2857 bracteosa Wats.
Chlorogalum 100\%

$285^{8}$ angustifolium Kellogg

2859 parviflorum Wats.

2860 purpureum Brandegee

286I pomeridianum (Ker) Kunth

Odontostomum 1009

2862 Hartwegii Torr.

Hemerocallis 1019 2863 fulva L.

\section{Leucocrinum 10\%\%}

2864 montanum Nutt.

\section{Hesperocallis 1023}

2865 undulata A. Gray

\section{Allium 1049}

2866 acuminatum Hook.

2867 Allegheniense Small

2868 anceps Kellogg

2869 arenicola Small

2870 atrorubens Wats.

$287 \mathrm{I}$ attenuifolium Kellogg

2872 Bidwelliae Wats.

2873 Bigelovii Wats.

2874 biseptrum Wats.

2875 Bolanderi Wats.

2876 Brandegei Wats.

2877 brevistylum Wats.

2878 Breweri Wats.

2879 campanula Wats.

2880 Canadense L.

288 I cernuum Roth

2882 obtusum Cockerell

2883 collinum Dougl.

2884 crenulatum Wiegand

2885 crispum Greene

2886 cristatum Wats.

2887 Cusickii Wats.

2888 dichlamydeum Greene

2889 Douglasii Hook.

2890 falcifolium H. \& A.

289 I fibrosum Rydb.

2892 fimbriatum Wats.

2893 Geyeri Wats.

2894 haematochiton Wats.

2895 Hendersoni Rob. \& Seaton 

2896 hyalinum Curran 2897 lacunosum Wats. 2898 I.emmoni Wats. 2899 macrum Wats. 2900 madidum Wats. 290 I monospermum Jepson 2902 mutabile Michx. 2903 Neo-Mexicanum Rydb. 2904 Nevadense Wats. 2905 Nevii Wats. 2906 Nuttallii Wats. 2907 obtusum Lemmon 2908 Palmeri Wats. 2909 Parishii Wats. 29Io Parryi Wats. 29II parvum Wats. 2912 peninsulare Lemmon 29I3 platycaule Wats. 2914 pleianthum Wats. 2915 Plummerae Wats. 2916 recurvatum Rydb. 2917 reticulatum Don. 2918 Sanbornii Wats. 2919 scaposum Benth. 2920 Schoenoprasum L. 292 I scilloides Dougl. 2922 serratum Wats. 2923 Sibiricum I. 2924 simillimum Henderson 2925 stellatum Ker 2926 stenanthum. Drew 2927 Tolmiei Baker 2928 tribracteatum Torr. 2929 tricoccum Ait. 2930 unifolium Kellogg 2931 lacteum Greene 2932 validum Wats. 2933 vineale $L$.

\section{Nothoscordum 1050}

2934 bivalve (I.) Britton (N. striatum Kunth)

\section{Muilla 1051}

2935 coronata Greene 2936 maritima Wats. 2937 trans-montana Greene

\section{Bloomeria 105\%}

2938 aurea Kellogg

2939 Clevelandi Wats.

2940 crocea (Torr.) Coville

294I montana Greene

\section{Brodiaea 1053}

2942 capitata Benth.

2943 congesta Smith

2944 grandifiora Smith

2945 major Benth.

2946 Hendersoni Wats.

2947 insularis Greene

2948 lactea Wats.

2949 multiflora Benth.

2950 pulchella (Salisb.) Greene

295 I volubilis Baker

\section{Hookera (1053)}

(Brodiaea in part)

2952 Californica (Lindl.) Greene

2953 coronaria Salisb.

2954 filifolia (Wats.) Greene

2955 minor (Benth.) Britten

2956 Orcuttii Greene

2957 rosea Greene

$295^{8}$ stellaris (Wats.) Greene

2959 terrestris (Kellogg) Greene

\section{Tritelia (1053)}

(Brodiaea in part)

2960 Bridgesii (Wats.) Greene

296I candida Greene

2962 crocea (Wood) Greene

2963 gracilis (Wats.) Greene

2964 grandiflora Lindl.

(Brodiaea Douglasii Wats.)

2965 Hendersoni Greene

2966 Howellii (Wats.) Greene

2967 hyacinthina (Lindl.) Greene

2968 ixioides (Ait. f.) Greene

2969 laxa Benth.

2970 Lemmonae (Wats.) Greene

297 I lilacina (Wats.) Greene

2972 lugens Greene

2973 peduncularis Lindl.

\section{Androstephium}

1055

2974 breviflorum Wats. 

2975 coeruleum (Scheele) Greene 2976 violaceum Torr.

\section{Milla 1061}

2977 biflora Cav.

\section{Stropholirion $106 \%$}

2978 Californicum Torr.

\section{Brevoortia 1063}

2979 venusta Greene

\section{Lilium 10\%?}

2980 Bolanderi Wata.

298I Canadense L.

2982 Carolinianum Michx.

2983 Catesbaei Walt.

2984 Columbianum Hansen

2985 Grayi Wats.

2986 Humboldtii Roezl \& Leicht.

2987 maritimum Kellogg

2988 angustifolium Kellogg

2989 montanum Aven Nelson

2990 occidentale Purdy

299 I pardalinum Kellogg

2992 Parryi Wats.

2993 parvum Kellogg

2994 luteum Kellogg

2995 Philadelphicum L.

2996 pulchrum Aldrich

2997 Purdyi Waugh (L. Bakeri Purdy)

2998 rubescens Wats.

2999 superbum L.

3000 tigrinum Andr.

300I umbellatum Pursh

3002 Washingtonianum Kellogg

\section{Fritillaria 10\%4}

3003 atropurpurea Nutt.

3004 biflora Lindl.

3005 coccinea Greene

3006 Kamtschatcensis Ker

3007 lanceolata Pursh

3008 floribunda Benth.

3009 gracilis Wats.

30IO liliacea Lindl.

30I I linearis Coult. \& Fisher

3012 parviflora Torr.
3013 pudica (Pursh) Spreng.

3014 recurva Benth

\section{Erythronium 10\%6}

3015 albidum Nutt.

3016 Americanum Ker

3017 citrinum Wats.

3018 giganteum Lindl.

3019 grandiflorum Pursh

3020 parviflorum Wats.

3021 Hartwegi Wats.

3022 Hendersoni Wats.

3023 Howellii Wats.

3024 mesachoreum Knerr

3025 montanum Wats.

3026 propullans A. Gray

3027 purpurascens Wats.

3028 revolutum Smith

3029 Bolanderi Wats.

\section{Lloydia 10\%'}

3030 serotina (L.) Sweet

\section{Calochortus $10 \% 8$}

303I acuminatus Rydb.

3032 albus Dougl.

3033 paniculatus Baker

3034 rubellus Greene

3035 amoenus Greene

3036 apiculatus Baker

3037 aureus Wats.

$303^{8}$ Benthami Baker

3039 caeruleus Wats.

3040 Catalinae Wats.

304I ciliatus Rob. \& Seaton

3042 clavatus Wats.

3043 caulinus Lemmon

3044 elegans Pursh

3045 nanus Wood

3046 eurycarpus Wats.

3047 excavatus Greene

3048 flexuosus Wats.

3049 Greenei Wats.

3050 Gunnisoni Wats.

3051 immaculatus Cockerell

3052 maculatus Cockerell

3053 purus Cockerell

3054 Howellii Wats.

3055 invenustus Greene 

3056 Kennedyi Porter

3057 lilacinus Kellogg

$305^{8}$ lougebarbatus Wats.

3059 luteus Dougl.

3060 citrinus Wats.

306I oculatus Wats.

3062 Lyoni Wats.

3063 macrocarpus Dougl.

3064 Maweanus Leicht.

3065 nitidus Dougl.

(C. pavonaceus Fernald)

3066 nudus Wats.

3067 Nuttallii T. \& G.

3068 Obispoensis Lemmon

3069 Palmeri Wats.

3070 Plummerae Greene

307I pulchellus Dougl.

3072 maculosus Wats.

3073 Purdyi Eastwood

3074 splendens Dong1.

3075 Tolmiei H. \& A.

3076 uniflorus H. \& A.

3077 venustus Benth.

3078 Weedii Wood

\section{Quamasia 108\%}

(Camassia)

3079 azurea Heller

3080 Cusickii (Wats.) Coville

308I esculenta (Ker) Coville

(Camassia Fraseri Torr.)

3082 Howellii (Wats.) Coville

3083 Leichtlinii (Baker) Coville

3084 Quamash (Pursh) Coville

(Camassia esculenta of authors)

Ornithogalum 1089

3085 nutans L.

3086 umbellatum L.

\section{Muscari 1095}

3087 botryoides (L.) Mill.

3088 racemosum (L.) Mill.

Yucca 1103

3089 arborescens (Torr.) Trelease ( $Y$. brevifolia Engelm.)

3090 aloifolia L.

3091 australis (Engelm.) Trelease
3092 baccata Torr.

3093 brevifolia Torr.

3094 filamentosa L.

3095 bracteata Engelm.

3096 flaccida Engelm.

3097 laevigata Engelm.

3098 glauca Nutt.

( $Y$. angustifolia Pursh)

3099 stricta (Sims) Trelease

3 Ioo gloriosa L.

3IOI Hanburii Baker

3IO2 macrocarpa ('Torr.) Coville

3103 Mohavensis Sargent

3IO4 radiosa (Engelm.) Trelease

3 IO5 rnpicola Scheele

3 io6 rigida Engelm.

3I07 Treculeana Carr.

3 108 Whipplei Torr.

\section{Hesperaloe 1104}

3rog Davyi Baker

3I Io Engelmanni Krausk.

3III yuccaefolia Engelm.

\section{Nolina 1105}

3II2 Bigelovii Wats

3II3 Brittoniana Nash

3II4 erumpens Wats.

3II5 Georgiana Michx.

3II6 Greenei Wats.

$3^{\text {II }} 7$ Lindheimeriana Wats.

3II8 microcarpa Wats.

3II9 Parryi Wats.

3I 20 Texana Wats.

Dasylirion 1106

3I2I Texanum Scheele

3I22 Wheeleri Wats.

\section{Schoenocaulon}

3I23 Drummondii A. Gray

3r24 gracile A Gray

Asparagus 1113

3I25 officinalis L.

Clintonia $111 \%$

3I 26 Andrewsiana Torr.

3127 borealis (Ait.) Raf. 

3I 28 umbellulata (Michx.) Torr. 3I 29 uniflora (Menzies) Kunth

\section{Vagnera 1118}

(Smilacina)

3I 30 amplexicaulis (Nutt.) Greene 3' ${ }^{\text {I I }}$ brachystyla (Henderson)

3I 32 liliacea (Greene) Rydb. Heller

3I 33 pallescens Greene

3I 34 racemosa (L.) Morong

3I 35 sessilifolia (Nutt.) Greene

3I 36 stellata (L.) Morong

3I 37 trifolia (L.) Morong

\section{Unifolium 1119}

(Maianthemum)

3I 38 Canadense (Desf.) Greene

\section{Disporum 1120}

3 I 39 Hookeri ('Torr.) B. \& H.

3I 4 O oblongifolium B. \& H.

3I 4 I lanuginosum (Michx.) Nichols 3I 42 maculatum (Buckley) B. \& H. 3I43 majus (Hook.) Britton (D. Oreganum B. \& H.)

3 I 44 Menziesii (Don.) B. \& H.

3I45 parviflorum B. \& H.

3I 46 trachyandrum ('Torr.) B. \& H. 3I 47 trachycarpum (Wats.) B. \& H.

\section{Streptopus 1121}

3148 amplexifolius (L.) DC.

3I49 brevipes Baker

3I 50 rosens Michx.

\section{Polygonatum 1123}

3I 5I biflorum (Walt.) Eill. 3 52 commutatum (R. \& S. Dietr. (P. giganteum Dietr.)

\section{Convallaria 1128}

3I53 majalis $\mathrm{L}$.

\section{Medeola 1135}

$3^{1} 54$ Virginiana L.

Scoliopus 1136

3I55 Bigelovii Torr. 3 I 56 Hallii Wats.

\section{Trillium 1138}

3I 57 cernutum L.

3 58 discolor Wray

3I59 erectum L.

3 I60 grandiflorum (Michx.) Salisb.

3r6I lanceolatum Boykin

3I62 nivale Riddell

3 r63 obovatum Pursh

3I64 ovatum Pursh

3165 petiolatum Pursh

3 66 pusillum Michx.

3 I.67 recurvatum Beck

3 I 68 rivale Wats.

3169 sessile $L_{1}$.

3r7o angustipetalum Torr.

3I7I Californicum Wats.

3I72 stylosum Nutt.

3I73 Underwoodii Small

3I74 undulatum Willd. (T. erythrocarpum Michx.)

3I 75 viride Beck.

\section{Aletris 1143}

3I76 aurea Walt.

$3 \mathrm{I} 77$ farinosa $\mathrm{L}$.

3I78 lutea Small

\section{SMILACEAE}

\section{Smilax 1151}

3I79 auriculata Walt.

3I80 Bona-nox L.

3I8I hederaefolia A. DC.

$3^{182}$ pandurata A. DC.

3 I83 polyodonta A. DC.

3I84 Wrightii A. DC.

3I 85 Californica A. Gray

3r86 cinnamomiifolia Small

3 I 87 diversifolia Small

3I88 ecirrhata (Engelm.) Wats.

3 i89 glauca Walt.

3I90 Havanensis Jacq.

3I9I herbacea I.

$3^{192}$ lasionenton A. DC.

3r93 longifolia A. DC.

3194 peduncularis A. DC.

3195 Simsii A. DC.

3I96 hispida Muhl.

3I97 lanceolata $\mathrm{L}$. 

3198 laurifolia L

3199 bupleurifolia Del.

3200 Morongii Small

(S. megacarpa Morong)

3201 ovata Pursh

3202 Buckleyi A. DC.

3203 Pseudo-China L.

3204 pumila Walt.

3205 renifolia Small

3206 rotuudifolia L.

3207 crenulata Small \& Heller

3208 Smallii Morong

3209 spinulosa J. E. Smith

32 Io tamnifolia Michx.

32 II tenuis Small

3212 Walteri Pursh

\section{HAEMADORACEAE}

\section{Gyrotheea 1161}

(Lachnanthes)

32 I 3 capitata (Walt.) Morong

(Lachnanthes tinctoria Ell.)

\section{AMARYLLIDACEAE}

Atamosco 1181

\section{(Zephyranthes)}

32 I4 Atamasco (L.) Greene

32 I 5 pulchella (J. G. Smith) Greene

3216 Simpsoni (Chapm.) Greene

3217 Texana (Herb.) Greene

3218 Treatiae (Wats.) Greene

\section{Cooperia 1183}

3219 Drummondii Herb.

3220 pedunculata Herb.

\section{Crinum 1189}

3221 Americanum L.

\section{Hymenocallis 1194}

3222 Caribaea Herb.

3223 Caymanensis Herb.

3224 coronaria Knnth

3225 crassifolia Herb.

3226 Galvestonensis Baker

3227 glauca Baker

3228 humilis Wats.

3229 lacera Salisb.
3230 paludosa Salisb.

323 I nutans B. \& H.

3232 occidentalis (LeConte) Kunth

3233 Palmeri Wats.

\section{Agave 1219}

3234 applanata Lemaire

3235 asperrima Jacobi

3236 decipiens Baker

3237 deserti Engelm.

$323^{8}$ Engelmanni Trelease

3239 Huachucensis Baker

3240 Lechuguilla Torr.

324I maculata Regel

3242 Newberryi Engelm.

3243 Palmeri Engelm.

3244 Parryi Engelm.

3245 parviflora Torr.

3246 rigida sisalina Engelm.

3247 Schottii Engelm.

3248 serrulata Mulford

3249 Shawii Engelm.

3250 Utahensis Engelm.

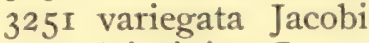

3252 Virginica L.

3253 tigrina Engelm.

\section{Hypoxis 1230}

3254 decumbens L.

3255 hirsuta (L.) Coville

(H. erecta L.)

3256 juncea Smith

\section{Lophiola 1240}

3257 Americana (Pursh) Coville (L. aurea Ker)

\section{DIOSCOREACEAE}

\section{Dioscorea 125\%}

$325^{8}$ villosa $\mathrm{L}$.

\section{IRIDACEAE}

Iris 1264

3259 bracteata Wats.

3260 Caroliniana Wats.

326I cristata Ait.

3262 Douglasiana Herb.

3263 fulva Ker 

3264 Germanica L.

3265 Hartwegi Baker

3266 australis S. B. Parish

3267 hexagona Walt.

3268 Hookeri Penny

3269 lacustris Nutt.

3270 longipetala Herb.

327 I macrosiphon Torr.

3272 Missouriensis Nutt.

3273 prismatica Pursh

3274 Pseudacorus L.

3275 Purdyi Eastwood

3276 setosa Pall.

3277 tenax Dougl.

3278 tenuis Wats.

3279 tripetala Walt.

3280 verna $L_{1}$.

$328 \mathrm{I}$ versicolor $\mathrm{L}$.

3282 Watsoniana Purdy

\section{Alophia 1269}

3283 caerulea B. \& H. 3284 Drummondiana Herb.

\section{Tigridia 12\%1}

3285 buccifera Wats.

\section{Nemastylis 12\%4}

3286 acuta (Bart.) Herb. ( $N$. geminiflora Nutt.)

3287 coelestina Nutt.

3288 purpurea Herb.

Calydora 1278

(Gelasine)

3289 Texana (Herb.) Baker

Gemmingia 1284

(Belamacanda)

3290 Chinensis (L.) Kuntze

\section{Sisyrinchium}

329 I albidum Raf.

3292 alpestre Bicknell

3293 angustifolium Mill.

3294 apiculatum Bicknell

3295 arenicola Bicknell

3296 Arizonicum Rothrock

3297 Asheianum Bicknell
3298 Atlanticum Bicknell

3299 bellum Wats.

3300 Californicum Ait. f.

33 I campestre Bicknell

$33 \mathrm{O}$ Kansanum Bicknell

3303 capillare Bicknell

3304 Carolinianum Bicknell

3305 corymbosum Bicknell

3306 demissum Greene

3307 dichotomum Bicknell

3308 Elmeri Greene

3309 Farwellii Bicknell

33 Io fiagellnm Bicknell

33 I I flaviflorum Bicknell

33 I2 flexile Bicknell

33I3 Floridanum Bicknell

33I4 furcatum Bicknell

33I5 fuscatum Bicknell

3316 geniculatum L.

33I7 graminoides Bicknell

(S. anceps of Gray's Manual, 6th Edition, not Cav.)

33 I 8 grandiflorum Dougl.

3319 halophilum Greene

3320 hastile Bicknell

332 I heterocarpum Bicknell

3322 Idahoense Bicknell

3323 implicatum Bicknell

3324 incrustatum Bicknell

3325 intermedium Bicknell

3326 Langloisii Greene

3327 leptocaulon Bicknell

3328 littorale Greene

3329 Macounii Bicknell

333 o membranaceum Bicknell

333I Miamiense Bicknell

3332 mucronatum Michx.

3333 nanum Bicknell

3334 Nashii Bicknell

3335 occidentale Bicknell

3336 rosulatum Bicknell

3337 rufipes Bicknell

$333^{8}$ sagittiferum Bicknell

3339 scabrellum Bicknell

3340 scoparium Bicknell

334I segetum Bicknell

3342 septentrionale Bicknell

3343 solstitiale Bicknell 

3344 strictum Bicknell

3345 tenellum Bicknell

3346 Thurowi Coult. \& Fisher

3347 tortum Bicknell

3348 Tracyi Bicknell

3349 versicolor Bicknell

3350 xerophyllum Greene

\section{CANNACEAE}

\section{Canna 1347}

335 I flaccida Roscoe

3352 Indica L.

\section{MARAN'TACEAE}

\section{Thalia 1358}

3353 dealbata Roscoe

3354 divaricata Chapm.

BURMA NNIACEAE

\section{Apteria 136\%}

3355 setacea Nutt.

\section{Burmannia 1363}

3356 biflora L.

3357 capitata Chapm.

\section{ORCHIDACEAE}

\section{Cypripedium 1369}

$335^{8}$ acaule Ait.

3359 arietinum $\mathrm{R}$. Br.

3360 Californicum A. Gray

336I candidum Willd.

3362 fasciculatum Kellogg

3363 guttatum Sw.

3364 hirsutum Mill.

(C. pubescens Willd.)

3365 montanum Dougl.

3366 parviflorum Salisb.

3367 passerinum Rich.

3368 reginae Walt.

$$
\text { (C. spectabile Salisb.) }
$$

\section{Orchis 13\%}

3369 rotundifolia Pursh 3370 spectabilis $\mathrm{L}$.
Habenaria 1398

337 I albida R. Br.

3372 blephariglottis (Willd.) Torr.

3373 holopetala (Lindl.) A. Gray

3374 borealis Cham.

3375 bracteata (Willd.) R. Br.

3376 brevifolia Greene

3377 ciliaris (L.) R. Br.

3378 clavellata (Michx.) Spreng.

(H. tridentata Willd.)

3379 conspicua Nash

3380 Cooperi Wats.

$33^{81}$ cristata (Michx.) R. Br.

$33^{82}$ dilatata (Pursh) Hook.

$33^{8} 3$ dilatatiformis Rydb.

3384 distans Griseb.

3385 elegans Bolander

3386 flagellaris Wats.

3387 fiava (L.) A. Gray (H. virescens Willd.)

3388 Garberi Porter

3389 grandiflora (Bigel.) Torr.

(H. fimbriata of Gray's Manual, not $\mathrm{R}$. Br.)

3390 Hookeriana A. Gray

339I hyperborea (L.) R. Br.

3392 integra (Nutt.) Spreng.

3393 lacera (Michx.) R. Br.

3394 leucophaea (Nutt.) A. Gray

3395 leucostachys (Lind1.) Wats.

3396 macroceratitis Willd.

3397 maritima Greene

3398 Michanxii Nutt.

3399 nivea (Nutt.) Spreng.

3400 obtusata (Pursh) Richards

340 I odontopetala Reichb. f.

3402 orbiculata (Pursh) Torr.

3403 pedicellata Wats.

3404 peramoena A. Gray

3405 psycodes (L.) A. Gray

3406 repens Nutt.

3407 sparsiflora Wats.

3408 stricta (Lindl.) Rydb.

3409 Thurberi A. Gray

34IO Unalaschensis Wats.

\section{Pogonia 1440}

34I I affinis Austin 

34 I 2 divaricata (L.) R. Br.

34 I3 ophioglossoides (L.) Ker

34 I 4 trianthophora (Sw.) B. S. P. (C. pendula Lindl.)

34I 5 verticillata (Willd.) Nutt.

Arethusa 1449

34 I6 bulbosa I.

Vanilia 1455

34 I7 planifolia Andr.

\section{Cephalanthera 1456} 3418 Austinae (A. Gray) Heller (C. Oregana Reichb.)

\section{Epipactis $145 \%$}

3419 gigantea Dougl.

3420 viridiflora (Hoffm.) Reichb.

(E. Helleborine of Gray's Manual, not Crantz.)

\section{Gyrostachys 1465}

(Spiranthes)

342 I brevifolia (Chapm.) Kuntze

3422 brevilabris (Lindl.) Kuntze

3423 cernua (L.) Kuntze

3424 cinnabarina (Llave) Kuntze

3425 gracilis (Bigel.) Kuntze

3426 Madrensis (Reichb. f.) Kuntze

3427 odorata (Nutt.) Kuntze

3428 ovalis (Lindl.) Kuntze

3429 plantaginea (Raf.) Britton

(S. latifolia Torr.)

3430 potrifolia (Lind1.) Kuntze

$343^{I}$ praecox (Walt.) Kuntze

$343^{2}$ Romanzoffiana (Cham.) MacM

3433 simplex (A. Gray) Kuntze

3434 stricta Rydb.

(Spiranthes Romanzoffiana of Gray's Manual, not Cham.)

\section{Stenorrhynchus 1468}

3435 Jaliscana (Wats.) Nash

\section{Listera 1469}

3436 auriculata Wiegand

3437 australis Lindl.

3438 borealis Morong

3439 caurina Piper
3440 convallarioides (Sw.) Torr,

344 I cordata (L.) R. Br.

3442 nephrophylla Rydb.

3443 Smallii Wiegand

(L. reniformis Small)

\section{Physurus 1473}

3444 querceticola Lindl.

\section{Peramium 1479}

(Goodyera)

3445 Menziesii (Lindl.) Morong 3446 pubescens (Willd.) MacM. 3447 repens (L.) Salisb.

3448 ophioides (Fernald) Heller 3449 tesselatum (Lodd.) Heller

\section{Ponthieva 1504}

3450 glandulosa R. Br.

\section{Achroanthes 1523}

(Microstylis)

345 I corymbosa (Wats.) Greene

$345^{2}$ Floridana (Chapm.) Greene

3453 monophylla (L.) Greene

3454 montana (Rothrock) Greene

3455 unifolia (Michx.) Raf.

(M. ophioglossoides Nutt.)

\section{Leptorchis $15 \% 6$}

$$
\text { (Liparis) }
$$

3456 liliifolia (L.) Kuntze

3457 Loeselii (L。) MacM.

\section{Calypso 1529}

$345^{8}$ bulbosa (L.) Oakes

(C. borealis Salisb.)

3459 occidentalis (Holzinger) Heller

\section{Corallorhiza 1530}

3460 Bigelovii Wats.

346 I Corallorhiza (L.) Karst.

$$
\text { (C. innata R. Br.) }
$$

3462 Mertensiana Bong.

3463 micrantha Chapm.

3464 multiflora Nutt.

3465 odontorhiza (Willd.) Nutt.

3466 striata Lindl.

3467 Wisteriana Conrad 

Tipularia 153\%

3468 unifolia (Muhl.) B. S. P. (T. discolor Nutt.)

\section{Polystachya 1536}

3469 luteola Hook.

\section{Epidendrum $15 \%$}

3470 bidentatum L.

347 I cochleatum L.

3472 conopseum Ait.

3473 difforme Jacq.

3474 nocturnum L.

3475 rigidum Sw.

3476 Tampense L.

3477 venosum Lindl.

Limodorum 1583

(Calopogon)

3478 multiflorum (Lindl.) Mohr 3479 pallidum (Chapm.) Mohr 3480 parviflorum (Lindl.) Nash 348 I tuberosum I.

(C. pulchellus R. Br.)

Hexalectris 1584 3482 aphyllus (Nutt.) Raf. (Bletia aphylla Nutt.)

\section{Bletia 1594}

3483 verecunda Sw.

\section{Aplectrum 1596}

3484 spicatum (Walt.) B. S. P. (A. hiemale Nutt.)

\section{Cyrtopodium 1606}

3485 ecristatum Fernald 3486 punctatum Lindl.

Dendrophylax 1\%68 3487 Lindenii Benth.

\section{SAURURACEAE}

Saururus 1\%\% 3488 cernuus L.

Anemiopsis 1\%80

$$
\text { (Houttuynia) }
$$

3489 Californica H. \& A.
PIPERACEAE

\section{Peperomia 1\%88}

3490 leptostachya Chapm.

349I magnoliaefolia DC.

CASUARINACEAE

Casuarina $1 \% 94$

3492 equisetifolia Forst.

\section{JUGLANDACEAE}

\section{Juglans $1 \% 99$}

3493 Californica Wats.

3494 cinerea L.

3495 nigra L.

3496 rupestris Engelm.

\section{Hicoria 1800}

(Carya)

3497 alba (L.) Britton

(C. tomentosa Nutt.)

3498 aquatica (Michx, f.) Britton

3499 borealis Ashe

3500 Carolinae-septentrionalis Ashe

350 glabra (Mill.) Britton

$$
\text { (C. porcina Nutt.) }
$$

3502 hirsuta Ashe

3503 laciniosa (Michx. f.) Sargent (C. sulcata Nutt.)

3504 minima (Marsh.) Britton

(C. amara Nutt.)

3505 myristicaeformis (Michx. f.)

Britton

3506 odorata (Marsh) Sargent

(C. microcarpa Nutt.)

3507 ovata (Mill.) Britton

$$
\text { (C. alba Nutt.) }
$$

3508 Pecan (Marsh.) Britton

$$
\text { (C. olivaeformis Nutt.) }
$$

3509 villosa (Sargent) Ashe

3510 pallida Ashe

\section{MYRICACEAE}

\section{Myrica 1801}

35 II Californica Cham.

35I2 Carolinensis Mill.

3513 cerifera $\mathrm{L}$. 

35I4 Gale L.

35I5 Hartwegi Wats.

3516 inodora Bartr.

35 I7 pumila (Michx.) Small

Comptonia (1801)

35 I8 peregrina (L.) Coult. (Myrica asplenifolia Endl.)

\section{LEITNERIACEAE}

\section{Leitneria $180 \%$}

3519 Floridana Chapm.

SALICACEAE

\section{Populus 1803}

3520 acuminata Rydb.

$352 \mathrm{I}$ alba L.

3522 angustifolia James

3523 balsamifera $\mathrm{L}$.

3524 candicans (Ait.) A. Gray

$35^{25}$ deltoides Marsh

$$
\text { ( } P \text {. monilifera Ait.) }
$$

3526 occidentalis Rydb.

3527 Fremonti Wats.

3528 Wislizeni Wats.

3529 grandidentata Michx.

3530 heterophylla L.

353 I nigra $L$.

3532 tremuloides Michx.

3533 trichocarpa T. \& G.

3534 cupulata Wats.

\section{Salix 1804}

3535 adenophylla Hook.

3536 alba L.

3537 coerulea (J. E. Smith)

3538 vitellina (L.) Koch

3539 amygdaloides Anders.

3540 anglorum Cham.

$$
\text { (S. Brownii Bebb) }
$$

334I arbusculoides Anders.

3542 arctica Pall.

3543 obcordata Anders.

3544 argophylla . Nutt.

3545 argyrocarpa Anders.

3546 atra Rydb.

3547 Austinae Bebb
3548 Babylonica L.

3549 balsamifera (Hook.) Barratt

$355^{\circ}$ Barclayi Anders.

(S. commutata Bebb, and vars.)

355 I latiuscula Anders.

$355^{2}$ Barrattiana Hook.

3553 Tweedyi Bebb

3554 Bebbiana Sargent

(S. rostrata Rich.)

3555 Bolanderiana Rowlee

3556 Bonplandiana H. B. K.

3557 brachycarpa Nutt.

(S. stricta (Anders.) Rydb.)

$355^{8}$ Breweri Bebb

3559 Californica Bebb

3560 callicarpaea Trautv.

3561 candida Fluegge

3562 Chamissonis Anders.

3563 chlorophylla Anders.

3564 pycnocarpa Anders.

3565 cordata Muhl.

3566 angustata (Pursh) Anders.

3567 Watsoni Bebb

3568 curtifiora Anders.

3569 cyclophylla Rydb.

3570 desertorum Rich.

357 I diplodictya Trautv.

3572 discolor Muhl.

3573 eriocephala (Michx.)

Anders.

3574 prinoides (Pursh) Anders.

3575 Dodgeana Rydb.

3576 exigua Nutt.

(S. Neradensis Wats.)

3577 virens Rowlee

3578 fluviatilis Nutt.

Koch 3579 fragilis L.

3580 fuscescens Anders.

3581 Geyeriana Anders.

(S. macrocarpa Nutt.)

$35^{82}$ glacialis Anders.

3.583 glauca L.

$35^{84}$ glaucophylla Bebb

3585 glaucops Anders.

3586 Groenlandica(Anders.)L Lundst.

$35^{8} 7$ herbacea L.

3588 Hookeriana Barratt

3589 humilis Marsh. 

3590 interior Rowlee

(S. longifolia Muhl.)

359 I

Wheeleri Rowlee

3592 irrorata Anders.

3593 Labradorica Rydb.

3594 laevigata $\mathrm{Bebb}$

3595 angustifolia Bebb

3596 congesta Bebb

3597 lasiandra Benth.

3598 caudata (Nutt.) Sudw.

3599 lancifolia (Anders.) Bebb

3600 lasiolepis Benth.

360 I Lemmoni Bebb

3602 macrostachya Bebb

3603 sphaerostachya Bebb

3604 longipes Shuttlw.

(S. Wardii Bebb)

3605 lucida Muhl.

3606 lutea Nutt.

3607 Lyallii (Sargent) Heller

3608 Mackenzieana Barratt

3609 macrostachya Nutt.

36ro lencodendroides Rowlee

36r I melanopsis Nutt.

361 2 microphylla C. \& S.

36 I 3 Missouriensis Bebb

36 I 4 monticola Bebb

36r5 myrtillifolia Anders.

36 I6 myrtilloides L.

36I7 pedicellaris (Pursh)Anders.

36I 8 nigra Marsh.

36 rg falcata (Pursh) Torr.

3620 niphoclada Rydb.

362 I nivalis Hook.

3622 Nuttallii Sargent

(S. flavescens Nutt.)

3623 brachystachys (Benth.)

624 caprioides(Anders.) Sargent 3625 occidentalis Koch

3626 orbicularis Anders.

3627 ovalifolia Trautv.

3628 palaeoneura Rydb.

3629 Parishiana Rowlee

3630 pellita Anders.

363 I petiolaris J. E. Smith

3632 gracilis Anders.

3633 petrophila $R y d b$.
3634 phlebophylla Anders.

3635 phylicifolia L.

3636 phylicoides Anders.

3637 Piperi Bebb

$363^{8}$ polaris Wahl.

3639 prolixa Anders.

3640 pseudomyrsinites Anders.

364I purpurea L.

3642 reticulata $\mathrm{L}$.

3643 Richardsonii Hook.

3644 Macouniana Bebb

3645 rotundifolia Trautv.

3646 Rydbergii Heller

3647 saximontana Rydb.

3648 sericea Marsh.

3649 'sessilifolia Nutt.

3650 Saskatchawana Seem.

$365^{1}$ Sitchensis Sanson

3652 Coulteri (Anders.) Bebb

3653 speciosa H. \& A.

3654 taxifolia H. B. K.

3655 tenera Anders.

3656 tenerrima (Henderson) Heller

3657 Thurberi Rowlee

3658 tristis Ait.

3659 Unalaschensis Cham.

3660 Uva-ursi Pursh

366 I venusta Anders.

3662 vestita Pursh

3663 viminalis $L$.

3664 Waghornei Rydb.

3665 Wolfii Bebb

\section{BETULACEAE}

Carpinus 1806

3666 Caroliniana Walt.

Ostrya 180'

3667 Knowltoni Coville

3668 Virginiana (Mill.) Willd.

\section{Corylus 1808}

3669 Americana Walt.

3670 Californica (A. DC.) Rose

367 I rostrata Ait.

\section{Betula 1809}

3672 glandulosa Michx.

3673 Kenaica W. H. Fivans 

3674 lenta L.

3675 lutea Michx. f.

3676 nana $L$.

3677 flabellifolia Hook.

3678 nigra L.

3679 occidentalis Hook.

3680 papyrifera Marsh.

368 I minor Tuckerman

3682 populifolia Marsh.

3683 pnmila L.

\section{Alnus 1810}

3684 acuminata H. B. K. (A. oblongifolia Torr.)

3685 Alnobetula (Ehrh.) K. Koch (A. viridis DC.)

3686 glutinosa (L.) Medic.

3687 incana (L.) Willd.

3688 maritima (Marsh.) Muhl.

3689 Oregana Nutt.

3690 rhombifolia Nutt.

3691 rugosa (Du Roi) K. Koch (A. serviata Willd.)

3692 sinuata (E. Regel) Rydb.

3693 tenuifolia Nutt.

(A. incana var, virescens Wats.)

\section{FAGACEAE}

\section{Fagus $181 \%$}

3694 Americana Sweet (E. ferruginea Ait.)

\section{Castanea 1813}

3695 dentata (Marsh.) Borkh.

(C. sativa var. Americana Michx.) 3696 nana Muhl.

3697 pumila (L.) Mill.

\section{Castanopsis (1813)}

3698 chrysophylla (Hook.) A. DC.

\section{Quercus 1815}

3699 acuminata (Michx.) Houba

(Muhlenbergii Engelm.)

3700 agrifolia Nee

3701 frutescens Engelm.

3702 alba L.

3703 Arizonica Sargent
3704 berberidifolia Lieb.

3705 brevifolia (Lam.) Sargent (cinerea Michx.)

3706 breviloba ('Torr.) Sargeut

3707 Breweri Engelm.

3708 Brittoni Davis

3709 Californica ('Torr.) Cooper

37 Io Catesbaei Michx.

37II Chapmani Sargent

(obtusiloba var. parvifolia Chapm.)

37 I 2 chrysolepis Lieb.

37r3 Palmeri Engelm.

37 I4 vaccinifolia (Kellogg)

Engelm.

3715 coccinea Wang.

37 I6 densifora H. \& A.

37 I 7 echinoides (R. Br.) Sargent

37 I 8 digitata (Marsh.) Sudw.

(falcata Michx.)

37 I9 Douglasii H. \& A.

3720 dumosa Nutt.

372 I munita Greene

3722 revoluta Sargent

3723 ellipsoidalis E. J. Hill

3724 Emoryi Torr.

3725 Engelmanni Greene

3726 elegantula Greene

3727 Gambelii Nutt.

3728 Garryana Dougl.

3729 geminata Small

3730 Georgiana M. A. Curtis

373I heterophplla Michx. f.

3732 hypoleuca Engelm.

3733 imbricaria Michx.

3734 Jacobi R. Br.

3735 laurifolia Michx.

3736 Leana Nutt.

3737 lobata Nee

$373^{8}$ lyrata Walt.

3739 MacDonaldi Greene

3740 macrocarpa Michx.

374I Fisherii Hampton

3742 Hamptonii Hampton

3743 Marylandica Muench.

(nigra of authors, not L.)

3744 Michauxii Nutt.

3745 minima (Sargent) Small

(virens var. dentata Chapm.) 

3746 minor (Marsh.) Sargent (stellata Wang., and obtusiloba Michx.)

3747 Margaretta Ashe

3748 Morehus Kellogg

3749 myrtifolia Willd

3750 nana (Marsh.) Sargent

(ilicifolia Wang.)

$375^{I}$ nigra L.

(aquatica Walt.)

$375^{2}$ oblongifolia Torr.

3753 pagodaefolia (E11.) Ashe

3754 Palmeri Engelm.

3755 palustris Du Roi

3756 parvula Greene

3757 Phellos L.

$375^{8}$ platanoides (Lam.) Sudw.

(bicolor Willd.)

3759 prinoides Willd.

3760 Prinus L.

376 I pumila (Marsh.) Sudw.

3762 reticulata H. \& B.

3763 rubra L.

3764 runcinata A. DC.

3765 Sadleriana R. Br.

3766 sericea Willd.

(cinerea var. pumila of Chapm.)

3767 sinuata (Lam.) Walt.

3768 subimbricaria (DC.) Sudw.

3769 Texana Buckley

3770 tomentella Engelm.

377 I Toumeyi Sargent

3772 tridentata (DC.) Engelm.

3773 turbinella Greene

3774 undulata Torr.

(grisea and pungens Liebm.)

3775 Gunnisoni Engelm.

3776 velutina Lam.

(tinctoria Bartr.)

3777 Virginiana Mill.

$$
\text { (virens Ait.) }
$$

3778 maritima (Chapm.)Sargent

3779 Wislizeni A. DC.

3780 frutescens Engelm.

\section{ULMACEAE}

Ulmus 1818

378 I alata Michx.
3782 Americana L.

3783 aspera Chapm.

3784 crassifolia Nutt.

3785 pubescens Walt.

(U. fulva Michx.)

3786 racemosa Thomas

3787 serotina Sargent

\section{Planera 1819}

3788 aquatica (Walt.) J. F. Gmel.

3789 Gmelini Michx.

\section{Celtis 1820}

3790 Georgiana Small

379 I Helleri Small

3792 iguanaeus (Jacq.) Sargent

3793 Mississippiensis Bosc.

3794 occidentalis $\mathrm{L}$.

3795 pallida Torr.

3796 pumila Pursh

3797 reticulata Torr.

3798 Tala Gillies

\section{Trema 18\%4}

3799 micrantha B. \& H.

\section{MORACEAE}

\section{Morus 1834}

3800 alba L.

380 r celtidifolia H. B. K.

3802 nigra L.

3803 rubra L.

\section{Toxylon 1839}

(Maclura)

3804 pomiferum Raf.

(M. aurantiaca Nutt.)

Broussonetia 1843

3805 papyrifera (L.) Vent.

\section{Ficus $18 \% 4$}

3806 aurea Nutt.

3807 brevifolia Nutt.

3808 populnea Willd.

(F. pedunculata Willd.)

\section{Humulus 188\%}

3809 Lupulus L. . 

Cannabis 1883

38ro sativa I.

\section{URTICACEAE}

\section{Urtica 1884}

3811 Breweri Wats.

3812 Californica Greene

38I 3 cardiophylla Rydb.

38 I 4 chamaedryoides Pursh

3815 dioica Pursh

$38 \mathrm{I} 6$ gracilis Ait.

3817 holosericea Nutt.

38I8 Lyallii Wats.

38 I 9 urens L.

Hesperocnide 1885

3820 tenella Torr.

Urticastrum 1890

(Laportea)

382 I divaricatum (L.) Kuntze

(L. Canadensis Gaud.)

\section{Adicea 1894}

(Pilea)

$3^{822}$ pumila (L.) Raf.

\section{Boehmeria 1900}

3823 cylindrica (L.) Willd.

3824 scabra Porter

\section{Parietaria $191 \%$}

3825 debilis Forst. f.

3826 Floridana Nutt.

3827 Pennsylvanica Muhl.

\section{LORANTHACEAE}

Razoumof'skya $199 \%$

(Arceuthobium)

3828 Americana (Nutt.) Kuntze

3829 divaricata (Engelm.) Kuntze

3830 Douglasii (Engelm.) Kuntze

383I microcarpa (Engelm.) Kuntze

3832 minuta (Engelm.) Kuntze

3833 occidentalis (Engelm.) Kuntze

3834 pusilla (Peck) Kuntze

3835 robusta (Engelm.) Kuntze

3836 vaginata (Willd.) Kuntze

\section{Phoradendron 1995}

$3^{8} 37$ Bolleanum Eiche

3838 Californicum Nutt.

$3^{8} 39$ flavescens (Pursh) Nutt.

3840 orbiculatum Engelm.

384I villosum Engelm.

3842 juniperinum Engelm.

3843 Libocedri Engelm.

3844 macrophyllum (Engelm.)

Cockerell

\section{SANTALACEAE}

Buckleya 2011

3845 distichophylla (Pursh) Torr.

Nestronia (2011)

(Darbya)

3846 umbellula Raf.

(D. umbellulata A. Gray)

\section{Comandra 2014}

3847 livida Richards

3848 pallida A. DC.

3849 umbellata (L.) Nutt.

\section{Pyrularia 2015}

3850 pubera Michx.

OLACACEAE

Schoepfia 2024

$3^{8} 5^{\text {I }}$ Schreberi Lam.

Ximenia 2033

3852 Americana L.

\section{ARISTOLOCHIACEAE}

\section{Asarum 2063}

3853 acuminatum (Ashe) Bicknell

3854 arifolium Michx.

$3^{855}$ callifolium Small

3856 Canadense L.

3857 caudatum Lindl.

$385^{8}$ heterophyllum Ashe

3859 ochranthum Ashe

3860 Lemmoni Wats.

386r majus (Ducharte) Heller

(A. Hartwegi Wats.)

3862 Memmingeri Ashe 

3863 minus Ashe

3864 reflexum Bicknell

3865 ambiguum Bicknell

3866 Ruthii Ashe

3867 Shuttleworthii Britten \&

(A. macranthum Small)

Baker

3868 Virginicum L.

\section{Aristolochia 206y}

3869 brevipes Benth.

3870 acuminata Wats.

387x Californica Torr.

3872 Clematitis L.

3873 convolvulacea Small

3874 macrophylla Lam (A. Sipho L'Her.)

3875 longifolia Engelm. \& Gray

3876 Nashii Kearney

$3^{877}$ pentandra L.

3878 reticulata Nutt.

3879 Serpentaria L.

3880 tomentosa Sims

\section{RAFFLESIACEAE}

Apodanthes $20 \% 1$

388I Thurberi B. \& H.

POLYGONACEAE

Macounastrum $20 \%$

(Koeniga I., not Adans.)

3882 Islandicum (L.) Small

Pterostegia $20 \% 8$ 3883 drymarioides F. \& M.

Nemacaulis $\mathbf{2 0 8 0}$ 3884 denudata Nutt.

\section{Hollisteria 2081}

3885 lanata Wats.

Lastarriaea $\mathbf{2 0 8} 2$ 3886 Chilensis Remy

Chorizanthe 208:3 3887 Andersoni Parry 3888 brevicornu Torr.
3889 Breweri Wats.

3890 Californica A. Gray

3891 Clevelandi Parry

3892 corrugata T. \& G.

3893 cuspidata Wats.

3894 diffusa Benth.

$3^{895}$ Douglasii Benth.

3896 albens Parry

3897 Fernandiana Wats.

3898 fimbriata Nutt.

3899 insignis Curran

3900 laciniata Torr.

390 I leptoceris Wats.

3902 membranacea Benth.

3903 Nortoni Greene

3904 Orcuttiana Parry

3905 Palmeri Wats.

3906 Parryi Wats.

3907 perfoliata A. Gray

3908 polygonoides T. \& G.

3909 procumbens Nutt.

39 Io pungens Benth.

39 II nivea Curran

3912 rigida T. \& G.

39 I 3 robusta Parry

39r4 spinosa Wats.

3915 staticoides Benth.

3916 stellulata Benth.

3917 Thurberi Wats.

3918 cryptantha Curran

3919 uniaristata T. \& G.

3920 valida Wats.

392 I Watsoni T. \& G.

3922 Wheeleri Wats.

3923 Xanti Wats.

Acanthoseyphus 2083a

3924 Parishii (Parry) Small

\section{Oxytheca $\mathbf{2 0 8 5}$}

3925 caryophylloides Parry

3926 dendroides Nutt.

3927 hirtiflora (A. Gray) Greene

3928 luteola Parry

3929 perfoliata T. \& G.

3930 spergulina (A. Gray) Greene

393 I trilobata A. Gray

3932 Watsoni T. \& G. 



\section{Eriogonum 2086}

3933 Abertianum Torr.

3934 acaule Nutt.

3935 agninum Greene

3936 alatum Torr.

3937 Alleni Wats.

3938 andinum Nutt.

3939 androsaceum Benth.

3940 anemophilum Greene

394I angulosum Benth.

3942 annuum Nutt.

3943 apiculatum Wats.

3944 arborescens Greene

3945 aridum Greene

3946 aureum Jones

3947 ambiguum Jones

3948 glutinosum Jones

3949 Baileyi Wats.

3950 bicolor Jones

395I Bloomeri S. B. Parish

3952 brachyanthum Coville

3953 brachypodum T. \& G.

3954 caespitosum Nutt.

3955 campanulatum Nutt.

3956 capillare Small

3957 cernuum Nutt.

3958 tenue T. \& G.

3959 umbraticum Eastwood

3960 chrysocephalum A. Gray

396r cinereum Benth.

3962 citharaeforme Wats.

3963 clavatum Small

3964 clavellatum Small

3965 cognatum Greene

3966 compositum Doug1.

3967 coriaceum Coult. \& Fisher

3968 corymbosum Benth.

3969 Covillei Small

3970 croceum Small

397 I curvatum Small

3972 dasyanthemum T. \& G.

3973 Jepsoni Greene

3974 deflexum Torr.

3975 delicatulum Wats.

3976 densum Greene

3977 depauperatum Small

3978 deserticola Wats.

3979 dichotomum Dougl.
3980 divaricatum N11tt.

398I Douglasii Benth.

3982 dumosum Greene

3983 effusum Nutt.

3984 elatum Dougl.

3985 elegans Greene

3986 elongatum Benth.

3987 Esmeraldense Wats.

3988 fasciculatum Benth.

3989 flavum Nutt.

3990 flexum Jones

399I formosum (K. Brandegee)

3992 giganteum Wats.

T. S. Brandegee

3993 glandulosum Nutt.

3994 glaucum .Small

3995 Gordoni Benth.

3996 gracile Benth.

3997 gracillipes Wats.

4098 gracillimum Wats.

4099 grandis Greene

4000 gossypinum Curran

400I Harfordii Small

4002 Havardii Wats.

4003 Heermannii Dur. \& Hil.

4004 heracloides Nutt.

4005 hieracifolium Benth.

4006 Hookeri Wats.

4007 incanum T. \& G.

4008 inflatum Torr.

4009 insigne Wats.

40IO Jamesii Benth.

40 I I flavescens Wats.

4OI2 Jonesii Wats.

4Or3 Kelloggii A. Gray

4or 4 Kennedyi Porter

40I 5 Kingii T. \& G.

4016 lachnogynum Torr.

4017 latifolium Smith

40r8 latum Small

4019 Lemmoni Wats.

4020 Lobbii T. \& G.

$4 \mathrm{O}$ I lonchophyllum T. \& G.

4022 longifolium Nutt.

4023 longilobum Jones

4024 luteolum Greene

4025 marifolium 'T. \& G.

4026 Mearnsii Parry 

4027 microthecum Nutt. 4028 rigidum Eastwood 4029 minimum Small 4030 minutiflorum Wats. 403I Mohavense Wats. 4032 molestum Wats. 4033 molle Greene 4034 multiceps Nees 4035 multiflorum Benth. 4036 Nealleyi Coult. 4037 nidularium Coville $403^{8}$ nivale Canby 4039 niveum Dougl. 4040 nodosum Small 404I Nortoni Greene 4042 nudum Dougl. 4043 nutans T. \& G. 4044 ochrocephalum Wats. 4045 ochroleucum Small 4046 Ordii Wats.

4047 ovalifolium Nutt. 4048 pallidum Small 4049 Palmeri Wats. 4050 panduratum Wats. 405I Parishii Wats. 4052 Parryi A. Gray 4053 parvifolium Smith 4054 panciflorum Pursh 4055 pendulum Wats. 4056 pharnaceoides Torr. 4057 Piperi Greene 4058 Plumatella Dur. \& Hilg. 4059 polycladon Benth. 4060 polyphyllum Small 406r polypodum Small 4062 Pondii Greene 4063 Porteri Small 4064 Pringlei Coult. \& Fisher 4065 proliferum T. \& G. 4066 puberulum Wats. 4067 pulvinatum Small 4068 Purpusi T. S. Brandegee 4069 pusillum T. \& G. 4070 pyrolaefolium Hook. 407 I coryphaeum T. \& G. 4072 racemosum Nutt. 4073 reniforme 'Torr. 4074 comosum Jones
4075 robustim Greene 4076 rosulatum Small 4077 rotundifolium Benth 4078 rubescens Greene 4079 rubiflorum Jones 4080 salsuginosum Hook. 408I saxatile Wats. 4082 Shockleyi Wats. 4083 Simpsoni Benth. 4084 Siskiyonensis Small 4085 spathulatum A. Gray 4086 speciosum Drew 4087 spergulinum A. Gray 4088 sphaerocephalum Dougl. 4089 stellatum Benth. 4090 bahiaeforme Wats.

409I Stokesae Jones 4092 strictum Benth. 4093 subalpinum Greene 4094 suffruticoșum Wats. 4095 sulcatum Wats. 4096 taxifolium Greene 4097 tenellum Torr. 4098 tenue Sinall 4099 Texanum Coult. \& Fisher 4I00 Thomasii Torr.

4 IOI Thompsonae Wats.

4IO2 Thurberi Torr.

4 IO3 thymoides Benth.

4IO4 tomentosum Michx.

4I05 Torreyanum A. Gray

4I06 trichopodum Torr.

4IO7 trichotomum Small

4I08 trinervatum Small

4IO9 tripobum Greene

4IIO triste Wats.

4III truncatum T. \& G.

4II2 turbinatum Small

4II3 umbellatum Torr.

4II4 monocephalum T. \& G.

4 II 5 ursinum Wats.

4116 vagans Wats.

4II villiflorum A. Gray

4II 8 candidum Jones

4II9 vimineum Dougl.

$4 \mathrm{I} 2 \mathrm{O}$ caninum Greene

4I 2 I vineum Small

4122 virgatum Benth. 

4I 23 Watsoni T. \& G.

4I24 Wrightii Torr.

4 I 25 subscaposa Wats.

\section{Rumex 2088}

4I26 Acetosa L.

4127 Acetosella L.

4I28 altissimus Wood.

4I 29 Arizonicus Britton

4I 30 Berlandieri Meisner

4I 3 I Brittanica L.

4I 32 bucephalophorus L.

4I33 conglomeratus Murray

4I 34 crispus I.

4I35 densiflorus Osterhout

4I 36 fascicularis Small

4I 37 Floridanus Meisner

4I 38 Geyeri (Meisner) Trelease

4I39 hastatulus Muhl.

4I 40 hymenosepalus Torr.

4I4I lacustris Greene

4I42 Langloisii Small

4 I 43 obtusifolius L.

4I44 occidentalis Wats.

4I 45 nanus (Hook.) Trelease

4I46 Patientia L.

4I47 paucifolius Nutt.

4148 persicarioides L.

(R. maritimus of Gray's Manual, not L.)

4I49 pulcher L.

4I 50 salicifolius Weinm.

4I 5 I salinus Aven Nelson

4I 52 sanguinetus L.

4I 53 spiralis Small

4I54 subalpinus Jones

4I 55 venosus Pursh

$4^{I} 5^{6}$ verticillatus $\mathrm{L}$.

\section{Oxyria 2089}

4I 57 digyna (L.) Camptd.

\section{Polygonum 2094}

4 I 58 alpinum Allioni

4I 59 Alaskanum Small

4 r6o foliosum (Keller) Small 4I6I amphibium L.

$4 \mathrm{I} 62$ arifolium $\mathrm{L}$.

4I63 Austinae Greene 4r64 aviculare L.

4I65 Bellardi Allioni

4I66 Bidwelliae Wats.

4I67 bistortoides Pursh

(P. Bistoria of authors, not L.)

4168 linearifolium (Wats.) Small

4169 Bolanderi Brewer

4 I 70 Californicum Meisner

4I7I camporum Meisner

4I72 Careyi Olney

4I73 cilinode Michx.

4I74 Convolvulus L.

4 I75 cristatum Engelm. \& Gray

4176 Davisiae Brewer

4I77 Douglasii Greene

4178 montanum Small

4 I 79 dumetorum L.

4I80 emersum (Michx.) Britton

(P. Muhlenbergii Wats.)

4I8I Engelmanni Greene

$4 \mathrm{I} 82$ erectum L.

4 I 83 exsertum Sinall

4I84 fusiforme Greene

4I85 Greenei Wats.

4186 Hartwrightii A. Gray

4I87 hirsutum Walt.

4r88 glabrescens Meisner

4 I89 Hydropiper I.

4I9O hydropiperoides Michx.

4I9I Macounii Sinall

4I92 incarnatum E11.

4I93 Islandicum Meisner

4I94 Kelloggii Greene

4I95 lapathifolium L.

4196 incanum (Schmidt) Koch

4I97 nodosum (Pers.) Small

4I98 littorale Link

4199 Sitchense Small

4200 longistylum Small

4201 maritimum L.

$42 \mathrm{O} 2$ Meisnerianum Beyrichianum

(C. \& S.) Meisner

4203 Mexicanum Small

4204 minimum Wats.

4205 minus Hudson

4206 Newberryi Small

4207 Nuttallii Small

4208 Opelousanum Riddell 

4209 orientale L.

42 IO paronychia C. \& S.

42 I I paronychoides Small

42 I 2 Parryi Greene

42 I 3 Pennsylvanicum L.

42 I 4 Persicaria L.

42 I 5 persicarioides H. B. K.

4216 phytolaccaefolium Meisner

42 I 7 polygaloides Meisner

42 I 8 Portoricense Bertero

42 I9 punctatum Ell.

$$
\text { (P. acre H. B. K.) }
$$

4220 eciliatum Small

422 I leptostachyum (Meisner)

4222 robustior Small

4223 ramosissimum Michx.

4224 proliflcum Small

4225 Rayi Babington

4226 sagittatum L.

4227 Sawatchense Small

4228 scandens L.

4229 segetum H. B. K.

4230 setaceum Baldwin

423I Shastense Brewer

4232 spergulariaeforme Meisner

( $P$. coarctatum Dougl.)

4233 tenue Michx.

4234 unifolium Small

4235 Virginianum L.

4236 viviparum $L$.

4237 Watsoni Small

(P. imbricatum Nutt.)

4238 Zuccarinii Small

(P. cuspidatum Sieb. \& Zucc.)

\section{Fagopyrum 2095}

4239 Fagopyrum (L.) Karst. (F. esculentum Moench.)

4240 Tataricum (L.) Gaertn.

\section{Polygonella 2096}

424I Americana (F. \& M.) Small

4242 articulata (L.) Meisner

4243 brachystachya Meisner

4244 ciliata Meisner

4245 Croomii Chapm.

4246 gracilis (Nutt.) Meisner

4247 macrophylla Small
4248 polygama (Vent.) A. Gray

\section{Brumnichia 2099}

4249 cirrhosa Banks

\section{Coccolobis $\mathbf{2 1 0 \%}$}

(Coccoloba)

4250 laurifolia (Jacq.) Sargent (C. Floridana Meisner)

425I uvifera (L.) Sargent

\section{CHENOPODIACEAE}

Nitrophila 2109

4252 occidentalis Wats.

\section{Aphanisma 2113}

4253 blitoides Nutt.

\section{Chenopodium $\% 116$}

4254 album L.

4255 viride (L.) Moq.

4256 ambrosioides L.

4257 anthelminticum L.

$425^{8}$ atrovirens Rydb.

4259 Berlandieri Moq.

4260 Bonus-Henricus I.

426I Boscianum Moq.

4262 Botrys L.

4263 Californicum Wats.

4264 carinatum R. Br.

4265 cornutum ('Torr.) B. \& H.

4266 foetidum Schrad.

4267 Fremontii Wats.

4268 glaucum L.

4269 humile Hook.

4270 hybridum L.

427 I incanum (Wats.) Heller

4272 leptophyllum (Moq.) Nutt.

4273 oblongifolium Wats.

4274 subglabrum Wats.

4275 murale I.

4276 farinosum Wats.

4277 olidum Wats.

4278 polyspermum L.

4279 rubrum I.

4280 urbicum L.

Roubieva (2116)

428 I multifida (I..) Moq. 

Blitum (2116)

4282 capitatum I.

\section{Cycloloma 211\%}

4283 atriplicifolium (Spreng.)Coult. (C. platyphyllum Moq.)

\section{Monolepis 2118}

4284 Nuttalliana (R. \& S.) Engelm. 4285 pusilla Torr.

4286 spathulata A. Gray

\section{Atriplex 2122}

4287 acanthocarpa Wats. 4288. Alaskana Wats. 4289 arenaria Nutt. 4290 argentea Nutt. 429I Barclayana Dietr. 4292 bracteosa Wats. 4293 Breweri Wats. 4294 Californica Moq. 4295 canescens (Pursh) James 4296 confertifolia Wats. 4297 cordulata Jones 4298 cornuta Jones 4299 coronata Wats. 4300 corrugata Wats. 430 r Coulteri Dietr. 4302 cristata H. B. K. 4303 decumbens Wats. 4304 depressa Jepson 4305 dilatata Greene 4306 draconis Jones 4307 elegans Dietr. 4308 eremicola Osterhout 4309 expansa Wats. 43 IO fasciculata Wats. 43 I I fruticulosa Jepson 43 I 2 gracilliflora Jones 4313 Gmelini C. A. Meyer 43I 4 Greggii Wats. 43I 5 hastata L. 43 I 6 hortensis L. 4317 hymenelytra Wats. 43I 8 lapathifolia Rydb. 43 I9 lentiformis (Torr.) Wats. 4320 leucophylla Dietr. 432 I microcarpa Dietr. 4322 nodosa Greene
4323 Nuttallii Wats.

4324 oppositifolia Wats.

4325 orbicularis Wats.

4326 pabularis Aven Nelson

4327 Parishii Wats.

4328 Parryi Wats.

4329 patula L.

4330 phyllostegia ('Torr.) Wats.

433 I polycarpa Wats.

4332 Powellii Wats.

4333 pusilla Wats.

4334 radiata (Torr.) Conlt.

4335 rosea L.

4336 saccharina Wats.

4337 spicata Wats.

$433^{8}$ subdecumbens Jones

4339 Suckleyana (Torr.) Rydb. (A. Endolepis Wats.)

4340 Torreyi Wats.

$434 \mathrm{I}$ trinervata Jepson

4342 truncata ('Torr.) A. Gray

4343 tuberculata (Torr.) Coult.

4344 Tularensis Coville

4345 verna Jepson

4346 volutans Aven Nelson

4347 Wolfii Wats.

4348 zosteraefolia Wats.

\section{Suckleya 2123}

4349 Suckleyana ('Torr.) Rydb. (S. petiolaris A. Gray)

\section{Grayia 2124}

4350 Brandegei A. Gray

$435^{\text {I }}$ spinosa (Hook.) Moq. (G. polygaloides H. \& A.)

\section{Eurotia 2125}

4352 lanata (Pursh) Moq.

\section{Kochia 2133}

4353 Americana Wats.

4354 vestita Wats.

4355 Californica Wats.

4356 scoparia (L.) Roth

\section{Corispermum $\mathbf{2 1 3 8}$}

4357 hyssopifolium I.

$435^{8}$ nitidum Kit.

4359 villosum $\mathrm{Rydb}$. 

Spirostachys 2143

4360 occidentalis Wats.

Salicornia 2150

436 I ambigua Michx.

4362 Bigelovii Torr.

(S. mucronata Bigel.)

4363 herbacea L.

4364 rubra Aven Nelson

4365 subterminalis S. B. Parish

Sarcobatus (2150)

4366 vermiculatus (Hook.) Torr.

\section{Dondia $\mathbf{2 1 5 4}$}

(Suaeda)

4367 Americana (Pers.) Britton

4368 Californica (Wats.) Heller

4369 conferta Small

437 o depressa (Pursh) Britton

437 I erecta (Wats.) Heller

4372 diffusa (Wats.) Heller

4373 intermedia (Wats.) Heller

4374 linearis (E11.) Heller

4375 maritima (L.) Druce

4376 minutiflora (Wats.) Heller

4377 multiflora (Torr.) Heller

4378 occidentalis (Wats.) Heller

4379 suffrutescens (Wats.) Heller

\section{Salsola 2162}

4380 Kali L.

438I Tragus L.

AMARANTHACEAE

\section{Celosia $21 \%$}

4382 paniculata L.

\section{Amaranthus $\mathbf{2 1 8 4}$}

4383 albomarginatus U. \& B.

4384 Berlandieri (Moq.) U. \& B.

$43^{8} 5$ Bigelovii U. \& B.

4386 emarginatus (Torr.) U.\& B.

4387 blitoides Wats.

4388 densifolius U. \& B.

$43^{89}$ Reverchoni U. \& B.

4390 Blitum L.

439 I Californicus Wats.
4392 carneus Greene

4393 crispus (Lesp. \& Thev.) Braun

4394 deflexus L.

4395 fimbriatus Benth

4396 denticulatus U. \& B.

4397 graecizans L.

4398 pubescens U. \& B.

4399 Greggii Wats.

4400 hybridus L.

(A. chlorostachys Willd.)

4401 paniculatus (L.) U. \& B.

4402 hypochondriacus L.

4403 leucospermus Wats.

4404 lividus L.

4405 obovatus Wats.

4406 Palmeri Wats.

4407 polygonoides L.

4408 Powellii Wats.

4409 Pringlei Wats.

44IO pumilus Raf.

44II retroflexus $\mathrm{L}$.

44I 2 scleropoides U. \& B.

44I 3 spinosus L.

44I4 squarrulosus (A. Gray) U. \& B.

4415 Torreyi (A Gray) Benth.

4416 tristis $\mathrm{L}$.

44I7 urceolatus U. \& B.

44I8 Jonesii U. \& B.

4419 obcordatus U. \& B.

4420 venulosus Wats.

442 I viscidulus Greene

4422 Wrightii Wats.

\section{Acnida 2185}

4423 australis A. Gray

4424 cannabina L.

4425 australis U. \& B.

4426 Floridana Wats.

4427 ruscocarpa Michx.

4428 tamariscina (Nutt.) Wood

4429 concatentata (Moq.) U. \& B.

$443^{\circ}$ prostrata U. \& B.

443 I tuberculata (Moq.) U. \& B.

\section{Acanthochiton 2186}

4432 Wrightii Torr.

\section{Guilleminea 2204}

4433 illecebrcides H. B. K. 

Cladothrix 2205

4434 cryptantha Wats.

4435 lanuginosa Nutt.

4436 oblongifolia Wats.

4437 suffrutescens B. \& H.

\section{Froelichia $\mathbf{2 2 0 6}$}

$443^{8}$ Drummondii Moq.

4439 Floridana (Nutt.) Moq.

4440 gracilis Moq.

444 I interrupta Moq.

4442 cordata U. \& B.

4443 Texana Coult. \& Fisher

\section{Gossypianthus 2208}

4444 rigidiflorus Hook.

Alternanthera $\mathbf{2 2 0 9}$

4445 Achyrantha R. Br.

4446 flavescens Moq. 4447 pungens H. B. K.

Mogiphanes (2209)

4448 Floridana B. \& H.

4449 straminea Mart.

\section{Telanthera (2209)}

4450 maritima Moq.

445I polygonoides Moq.

Dicraurus 2211 $445^{2}$ leptocladus Hook. f.

Gomphrena 221\%

4453 caespitosa Torr.

4454 Nealleyi Coult. \& Fisher

4455 nitida Rothrock

4456 Sonorae Torr.

4457 tuberifera Torr.

Iresine 2\%13

$445^{8}$ paniculata (L.) Kuntze

(I. celosioides L.)

Lithophila $\mathbf{2 2 1 4}$

(Philoxerus)

4459 vermiculata (L.) Uline

BATIDACEAE

Batis 2215

4460 maritima L.
BASELLACEAE

\section{Anredera 2\%:1}

446I scandens Moq.

\section{PHYTOLACCACEAE}

\section{Rivina 222'}

4462 humilis L. 4463 laevis L.

Petiveria 2228 4464 alliacea L.

Phytolacea 223\% 4465 decandra L.

NYCTAGINACEAE

Hermidium $\mathbf{2 4 4}$

4466 alipes Wats.

\section{Selinocarpus $\mathbf{2 2 4 6}$}

4467 angustifolius A. Gray 4468 chenopodioides A. Gray

4469 diffusus A. Gray

4470 lanceolatus Wooton

Nyctaginia $224 \%$

447 I capitata Choisy

\section{Mirabilis $2 \% 48$}

4472 Bigelovii A. Gray

4473 Californica A. Gray

4474 coccinea Benth.

4475 Greenei Wats.

4476 Jalapa L.

4477 multiflora A. Gray

4478 pubescens Wats.

4479 oxybaphoides A. Gray

4480 tenuiloba Wats.

448I Wrightiana A. Gray

\section{Allionia (2248)}

$$
\text { (Oxybaphus) }
$$

4482 albida Walt.

4483 Bodini (Holzinger) Morong

4484 Bushii Britton

4485 diffusa Heller

4486 glabra (Wats.) Kuntze

4487 hirsuta Pursh 

4488 linearifolia (Wats.) Kuntze 4489 linearis Pursh

(Oxybaphus angustifolius Sweet) 4490 nyctaginea Michx.

449 r latifolia (A. Gray)

4492 pilosa (A. Gray)

4493 ovata Pursh

(O. nyctagineus var. oblongifolius Torr.)

4494 viscosa (Cav.) Kuntze

\section{Acleisanthes $\mathbf{2 2 4 9}$}

4495 anisophylla A. Gray

4496 Berlandieri A. Gray

4497 crassifolia A. Gray

4498 longiflora A. Gray

4499 Wrightii B. \& H.

\section{Boerhaavia 2250}

4500 anisophylla Torr.

45 O paniculata Coult. \& Fisher

$45 \mathrm{O} 2$ Coulteri Wats.

4503 decumbens Vahl.

4504 erecta I.

4505 erioselena A. Gray

4506 gibbosa Pavon.

4507 hirsuta Willd.

4508 linearifoliu A. Gray

4509 leiosolena A. Gray

45 IO paniculata Rich.

45 II pterocarpa Wats.

45 I2 purpurascens A. Gray

45 I 3 scandens $L$.

45 I 4 spicata Torreyana Wats.

$45^{1} 5$ tenuifolia A. Gray

$45 \mathrm{I} 6$ viscosa Lag.

4517 Wrightii A. Gray

\section{Wedelia 2251}

(Allionia)

45 I 8 incarnata (L.) Kuntze

\section{Abronia 2\%53}

45I 9 alba Fastwood

4520 alpina $T$. S. Brandegee

$45^{21}$ angulata Jones

4522 angustifolia Greene

$45^{23}$ arenaria Rydb.

4524 Carletoni Coult. \& Fîsher
$45^{2} 5$ carnea Greene

4526 Crux-Maltae Kellogg

$45^{27}$ cyoloptera A. Gray

4528 elliptica Aven Nelson

4529 fragrans Nutt.

4530 gracilis Benth.

453I latifolia Esch.

4532 maritima Nutt.

4533 mellifera Dougl.

4534 micrantha ('Torr.) Chois.

4535 nana Wats.

4536 pogonantha Heimer.

4537 Suksdorfii Coult. \& Fisher

4538 turbinata Torr.

4539 umbellata Lam.

4540 villosa Wats.

\section{Pisonia 2256}

454I aculeata L.

4542 obtusata Jacq.

4543 rotundata Griseb.

\section{AIZOACEAE}

\section{Mollugo 2260}

4544 Cervina Seringe

4545 verticillata $L$.

4546 scrobiculata Rohrb.

\section{Glinus 2261}

4547 Cambessidesii Fenzl.

4548 lotoides virens Fenzl.

\section{Sesuvium 226:}

4549 maritimum (Walt.) B. S. P. (S. pentandrum Ell.)

$455^{\circ}$ Portulacastrum I. 455 I sessile Pers.

\section{Trianthema 2:6s}

$455^{2}$ monogyna L.

\section{Cypselea 2:69}

4553 humifusa Turp.

\section{Mesembrianthemum $2 \%$}

4554 aequilaterale Haw.

4555 crystallinum L.

4556 nodiflorum Haw. 



\section{PORTULACACEAE}

\section{Talinum 2278}

4557 brevifolium Torr.

$455^{8}$ calycinum Engelm.

4559 humile Greene

4560 lineare H. B. K.

4561 parviflorum Nutt.

4562 patens Willd.

4563 sarmentosum (Engelm.)

4564 rugospermum Holzinger

A. Gray

4.565 spinescens Torr.

4566 teretifolium Pursh

\section{Calandrinia $22 \% 9$}

4567 ambigua (Wats.) Howell (Claytonia ambigua Wats.)

4568 Breweri Wats.

4569 caulescens H. B. K.

457 o Menziesii (Hook.) T. \& G.

4571 maritima Nutt.

\section{Oreobroma (2279)}

4572 brachycalyx (Engelm.) Howell (Lewisia brachycalyx Engelm.)

4573 Columbiana Howell

4574 cotyledon (Wats.) Howell

4575 Grayi (Britton) Rydb.

(Talinum pygmaeum A. Gray)

4576 Howellii (Wats.) Howell

4577 Leana (Porter) Howell

4578 minima Aven Nelson

4579 Nevadensis (A. Gray) Howell 4580 oppositifolia (Wats.) Howell 458 I triphylla (Wats.) Howell (Claytonia triphylla Wats.) 4582 Tweedyi (A. Gray) Howell

\section{Calyptridium $\mathbf{2 2 8 1}$}

4583 monandrum Nutt.

4584 Parryi A. Gray

$45^{8} 5$ quadripetalum Wats.

4586 roseum Wats.

Talinopsis $\mathbf{2 2 8 2}$

$45^{87}$ frutescens A. Gray

Claytonia $\mathbf{2 2 8 6}$

4588 arctica Adams
4589 anrea Aren Nelson

4590 Bodini Holzinger

4591 Caroliniana Michx.

4592 lanceolata Pursh

4593 sessilifolia Aven Nelson

4594 megarrhiza (A. Gray) Parry

4595 multicaulis Aven Nelson

4596 nubigena Greene

4597 sarmentosa C. A. Meyer

4598 saxosa Brandegee

4599 tuberosa Pall.

4600 umbellata Wats.

460I Virginica L.

\section{Montia $\mathbf{2 2 8 8}$}

(Claytonia in part)

4602 arenicola (Henderson Howell 4603 asarifolia (Bong.) Howell 4604 bulbifera (A. Gray) Howell 4605 Chamissonia (Esch.) Greene 4606 depressa (A. Gray) Rydb. 4607 dichotoma (Nutt.) Howell 4608 diffusa (Nutt.) Greene 4609 flagellaris (Bong.) Robinson 46ro fontana L.

46I I gypsophiloides (F. \& M.) Howell

46I 2 Hallii (A. Gray) Greene 46 I 3 Howellii Wats.

46r4 linearis (Dougl.) Greene 46I 5 parvifolia (Moq.) Greene 46r6 parviflora (Dougl.) Howell

46 I 7 perfoliata (Donn) Howell

46 I 8 rosulata Eastwood

4619 rubra Howell

4620 sarmentosa (C. A. Mever)

Robinson

462 I saxosa Brandegee

4622 Sibirica (L.) Howell

4623 heterophylla (A. Gray)

Howell

4624 spathulata (Dougl.) Howell

4625 exigua ('T. \& G.) Robinson

\section{Portulaca 229:3}

4626 coronata Small 4627 grandiflora Hook. 4628 halimoides L. 

4629 lanceolata Engelm.

4630 oleracea L.

463I parvula A. Gray

4632 pilosa L.

4633 retusa Engelm.

4634 stelliformis Moq. \& Sesse

Lewisia 2294

4635 Kelloggii K. Brandegee

4636 redeviva Pursh

4637 Yosemitana K. Brandegee

CARYOPHYLLACEAE

Agrostemma 2296

4638 Githago L.

(Lychnis Githago Lam.)

Viscaria 229\%

4639 alpina (L.) Roeh1.

Silene $\mathbf{2 2 9 8}$

4640 acaulis L.

464 I alba Muh1.

(S. nivea Otth)

4642 Anglica L.

4643 antirrhina L.

4644 divaricata Robinson

4645 linaria Wood

4646 Armeria L.

4647 Baldwinii Nutt.

4648 Bernardina Wats.

4649 Bridgesii Rohrb.

4650 Californica Durand

465 I subcordata Robinson

4652 campanulata Wats.

4653 Greenei Wats.

4654 orbiculata Robinson

4655 Caroliniana Walt.

(S. Pennsylvanica Michx.)

4656 conica L.

4657 Cucubalus Wibel

4658 dichotoma Ehrh.

4659 Douglasii Hook.

4660 brachycalyx Robinson

466I viscida Robinson

4662 Gormani Howell

4663 Grayi Wats.

4664 Greenei (Wats.) Howell

4665 Hallii Wats.
4666 Hookeri Nutt.

4667 incompta A. Gray

4668 laciniata Cav.

4669 Greggii (A. Gray) Wats.

4670 Lemmoni Wats.

467 I longistylis Engelm.

4672 Luisana Wats.

4673 Lyallii Wats.

\section{(S. Macounii Wats.)}

4674 macrocalyx (Robinson)Howell

4675 Menziesii Hook.

4676 monantha Wats.

4677 montana Wats.

4678 rigidula Robinson

4679 multicaulis Nutt.

4680 multinervia Wats.

468I noctiflora L.

4682 nocturna $L$.

4683 nutans $\mathrm{L}$.

4684 occidentalis Wats.

4685 Oregana Wats.

4686 ovata Pursh

4687 Palmeri Wats.

4688 Parishii Wats.

4689 pectinata Wats.

4690 subnuda Robinson

469I platyota Wats.

4692 plicata Wats.

4693 purpurata Greene

4694 quinquevulnera $\mathrm{L}$.

4695 rectiramea Robinson

4696 regia Sims

4697 repens Patrin

4698 rotundifolia Nutt.

4699 Sargentii Wats.

4700 scaposa Robinson

4701 Scouleri Hook.

$47 \mathrm{O} 2$ Shockleyi Wats.

4703 simulans Greene

4704 Spaldingii Wats.

4705 stellata (L.) Ait. f.

4706 subciliata Robinson

4707 Suksdorfli Robinson

4708 Thurberi Wats.

4709 verecunda Wats.

47 IO Virginica L.

47 I I vulgaris (Moench) Garcke

4712 Watsoni Robinson 

47I3 Wrightii A. Gray

Lychnis 2299

47I4 affinis Vahl.

47 I5 alba Mill.

4716 alpina L.

47 I 7 apetala L.

47 I 8 elatior Begel

4719 glabra Regel

4720 Californica Wats.

472 I Chalcedonica L.

4722 Coronaria (L.) Desr.

4723 dioica L.

(L. diurna Sibth.)

4724 Drummondii (Hook.) Wats.

4725 elata Wats.

4726 Flos-cuculi L.

4727 Kingii Wats.

4728 montana Wats.

4729 nuda Wats.

4730 Parryi Wats.

4731 Taylorae Robinson

4732 triflora R. Br.

4733 Dawsoni Robinson

\section{Gypsophila 2305}

4734 muralis L.

4735 paniculata $\mathrm{L}$.

\section{Tunica 2306}

4736 Saxifraga (L.) Scop.

\section{Vaccaria $\mathbf{2 3 0 8}$}

4737 Vaccaria (L.) Britton (Saponaria Vaccaria L.)

\section{Dianthus 2309}

4738 alpinus repens (Willd.) Regel

4739 Armeria L.

4740 barbatus L.

474 I deltoides L.

4742 prolifer L.

Saponaria $\mathbf{2 3 1 0}$

4743 officinalis $\mathrm{L}$.

Velezia 2311

4744 rigida L.

\section{Alsine 2312}

(Stellaria)

4745 Americana (Porter) Rydb.

4746 aquatica (L.) Britton

4747 Baicalensis Coville

(Stellaria umbellata Turcz.)

4748 borealis (Bigel.) Britton

4749 alpestris (Fries) Britton

4750 brachypetala (Bong.) Howell

$475^{I}$ calycantha (Ledeb.) Rydb.

4752 crassifolia (Ehrh.) Britton

4753 crispa C. \& S.) Holzinger

4754 dicranoides (C. \& S.) Heller

4755 fontinalis (S. \& P.) Britton

4756 graminea (L.) Britton

4757 Holostea (L.) Britton

4758 humifusa (Rottb.) Britton

4759 Jamesiana ('Torr.) Heller (Stellaria Jamesii Torr.)

4760 laeta (Rich.) Rydb.

476I littoralis ('Torr.) Greene

4762 longifolia (Muhl.) Britton

4763 longipes (Goldie) Coville

4764 Edwardsii (R. Br.) Britton

4765 peduncularis (Bunge)

4766 media L.

4767 nitens (Nutt.) Greene

4768 Nuttallii (T. \& G.) A. Gray

4769 obtusa (Engelm.) Rose

4770 oxyphylla (Robinson) Heller

477 I prostrata (Baldwin) Heller

4772 pubera (Michx.) Britton

4773 Simcoei Howell

4774 uliginosa (Murr.) Britton

4775 uniflora (Walt.) Heller

4776 Washingtoniana (Robinson)

Heller

\section{Cerastium 2313}

4777 alpinum L.

4778 Fischerianum (Ser.)'T.\& G.

4779 glabratum Hook.

4780 uniflorum Durand

$478 \mathrm{I}$ arvense $\mathrm{L}$.

4782 Fuegianum Hook.

4783 maximum Britton \& Holl. 

4784 oblongifolium (Torr.)

Britton \& Holl.

4785 velutinum (Raf.) Britton

4786 Behringianum C. \& S.

4787 brachypodum (Engelm.)

Robinson

4788 compactum Robinson

4789 Buffumae Aven Nelson

4790 cerastioides (L.) Britton

$$
\text { (C. trigynum Vill.) }
$$

479 I elongatum Pursh

4792 grande Greene

4793 longipedunculatum Muhl. (C. nutans Raf.)

4794 maximum $\mathrm{L}$.

4795 pilosum Ledeb.

4796 semidecandrum L.

4797 sericeum Wats.

4798 strictum L.

4799 Texanum Britton

4800 viscosum L.

480 tenellum Grenier

4802 vulgatum $\mathrm{L}$.

\section{Holosteum $\mathbf{2 3 1 4}$}

4803 umbellatum $\mathrm{L}$.

\section{Moenchia $\mathbf{2 3 1 5}$}

4804 erecta (L.) Gaertn.

\section{Sagina 2316}

4805 apetala Ard.

4806 crassicaulis Wats.

4807 decumbens (E11.) T. \& G.

4808 Smithii (A. Gray) Wats.

4809 nivalis Fries

48 Io nodosa (L.) Fenzl

48 I I occidentalis Wats.

48 I 2 procumbens $\mathrm{L}$.

48 I 3 saginoides (L.) Britton (S. Linnaei Pres1)

\section{Arenaria 2325}

48 I4 aculeata Wats.

48 I 5 alsinoides Willd.

$48 \mathrm{I} 6$ arctica Stev.

48I7 Benthamii Fenzl

48I 8 brevifolia Nutt.

48I9 Californica Brewer

4820 capillaris Poir.
482 I nardifolia (Ledeb.) Regel

4822 Caroliniana Walt.

4823 ciliata humifusa Hornem.

4824 compacta Coville

4825 congesta Nutt.

4826 Parishiorum Robinson

4827 suffrutescens (A. Gray)

Robinson

4828 Douglasii Fenzl

4829 Fendleri A. Gray

4830 diffusa Porter \& Coult.

483 I glabrescens Wats.

4832 formosa Fisch.

4833 Franklinii Dougl.

4834 glabra Michx.

4835 Groenlandica (Retz) Spreng.

4836 Hookeri Nutt.

4837 Howellii Wuts.

4838 Kingii (Wats.) Jones

4839 laricifolia L.

4840 macradenia Wats.

484I macrocarpa Pursh

4842 Michauxii Fenzl

4843 Nuttallii Pax

$$
\text { (A. pungens Nutt.) }
$$

4844 gracilis Robinson

4845 paludicola Robinson

4846 palustris Wats.

4847 patula Michx.

4848 physodes Fisch.

4849 pinetorum Aven Nelson

4850 propinqua Richards

485 I pumicola Coville \& Leiberg.

4852 pusilla Wats.

4853 Rossii Richards

4854 Rusbyi Greene

4855 Sajanensis Willd.

4856 carnosula (Fenzl)Robinson

4857 rigidula (Fenzl) Robinson

$485^{8}$ Salmonensis Henderson

4859 saxosa A. Gray

4860 cinerascens Robinson

486I scabrella Greene

4862 serpyllifolia L.

4863 tenuior Koch

4864 stricta Michx.

(A. Michauxii Hook. f.)

4865 subcongesta (Wats.) Rydb. 

4866 lithophila Rydb. 4867 tenella Nutt.

4868 Texana (Robinson) Britton

4869 Uintahensis Aven Nelson

4870 ursina Robinson

487 I verna $\mathrm{L}$.

4872 equicaulis Aven Nelson

4873 rubella Hook. f.

\section{Ammodenia (2325)}

(Arenaria in part)

4874 major (Hook.) Heller

4875 peploides (L.) Rupr.

\section{Moehringia $\mathbf{2 3 \% 6}$}

(Arenaria in part)

4876 lateriflora (L.) Fenzl

4877 macrophylla (Hook.) Torr.

\section{Spergula $\mathbf{2 3 3 1}$}

4878 arvensis $L_{\text {. }}$.

\section{Tissa 2332}

\section{(Buda; Spergularia)}

4879 Canadensis (Pers.) Britton (Buda borealis Wats.)

4880 Clevelandi Greene 488I diandra (Boiss.) Britton 4882 gracilis (Wats.) Britton 4883 letcantha Greene 4884 macrotheca (F. \& M.) Britton 4885 scariosa Britton 4886 marina (L.) Britton 4887 pallida Greene 4888 rubra (L.) Britton 4889 perennans (Kindb.) Greene 4890 sparsiflora Greene 489 I tenuis Greene 4892 valida Greene

\section{Drymaria $\mathbf{2 3 3 4}$}

4893 cordata Willd. 4894 effusa A. Gray 4895 Fendleri Wats. 4896 holosteoides Benth. 4897 sperguloides A. Gray 4898 tenella A. Gray

Polycarpon $\mathbf{2 3 3 5}$ 4899 depressum Nutt.
4900 tetraphyllum L.f.

\section{Stipulicida 2339}

4901 filiformis Nash 4902 setacea Michx.

\section{Loeflingia 2340}

4903 pusilla Curran 4904 squarrosa Nutt. 4905 Texana Hook.

\section{Achyronichia $\mathbf{2 3 4}$}

4906 Cooperi A. Gray 4907 Rixfordii Brandegee

\section{Paronychia $\mathbf{2 3 5 \%}$}

4908 argyrocoma (Michx.) Nutt.

4909 Baldwinii Fenzl

49Io Chilensis DC.

49I I chorizanthoides Small

49 I 2 dichotoma (L.) Nutt.

4913 diffusa Aven Nelson

4914 Drummondii T. \& G.

49 I 5 hernarioides (Michx.) Nutt.

49I6 Jamesii T. \& G.

4917 depressa Nutt.

4918 Lindheimeri Engelin.

49I9 pulvinata A. Gray

4920 pusilla Greene

492 I riparia Chapm.

4922 scoparia Small

4923 sessiliflora Nutt.

4924 brevicuspis Aven Nelson

4925 setacea T. \& G.

\section{Anychia $\mathbf{2 3 5 3}$}

4926 Canadensis (I.) B. S. P. (A. capillacea DC.)

4927 dichotoma Michx.

\section{Siphonychia $\mathbf{2 3 5 5}$}

4928 Americana T. \& G.

4929 corymbosa Small

4930 diffusa Chapm.

493I erecta Chapm.

Gibbesia 2355a

4932 Rugelii (Chapm.) Small (Siphonychia Rugelii Chapm.)

\section{Scleranthus 2361}

4933 annuus $\mathrm{L}$. 



\section{Pentacaena}

4934 polycnemoides Bartl.

NYMPHAEACEAE

\section{Nelumbo $\mathbf{2 3 6 5}$}

4935 lutea (Willd.) Pers. 4936 Nelumbo (L4.) Karst.

\section{Cabomba $\quad 2366$}

4937 Caroliniana A. Gray

Brasenia 236y $493^{8}$ purpurea (Michx.) Casp. (B. peltata Pursh)

\section{Castalia 2370}

(Nymphaea)

4939 ampla (DC.) Greene 4940 elegans (Hook.) Greene 494I flava (Light.) Greene 4942 odorata (Dryand)

Woodv. \& Wood.

4943 rosea (Pursh) Britton 4944 reniformis (Walt.) Nash 4945 tetragona (Georgi) Lawson (C. Leibergii Morong) 4946 tuberosa (Paine) Greene

Nymphaea $23 \% 1$

$$
\text { (Nuphar) }
$$

4947 advena Soland. 4948 hybrida Peck

(N. rubrodisca (Morong) Greene) 4949 Kalmiana (Michx.) Sims 4950 macrophylla Small 495 I orbiculata Small $495^{2}$ polysepala (Engelm.) Greene 4953 sagittaefolia Walt.

\section{CERATOPHYLLACEAE}

Ceratophyllum $\mathbf{2 3 7 3}$ 4954 demersum L. 4955 echinatum A. Gray

\section{MAGNOLIACEAE}

\section{Magnolia $\mathbf{2 3 \% 4}$}

4956 acuminata L.
4957 cordata Michx. $495^{8}$ foetida (L.) Sargent (M. grandiflora L.)

4959 Fraseri Walt. 4960 macrophylla Michx. 496r tripetala L. (M. Umbrella Lam.)

4962 Virginiana L. (M. glauca L.)

\section{Liriodendron $23 \%$} 4963 Tulipifera L.

\section{Schizandra $23 \% 9$} 4964 coccinea Michx.

\section{Illicium $\mathbf{2 3 8 0}$}

4965 Floridanum Ell. 4966 parviflorum Vent.

\section{ANNONACEAE}

\section{Asimina $\mathbf{2 3 9 9}$}

4967 angustifolia A. Gray 4968 obovata (Willd.) Nash 4969 parviflora (Michx.) Dunal 4970 pygmaea (Bartr.) A. Gray 497 I reticulata Shuttlw.

4972 Rugelii Robinson 4973 speciosa Nash 4974 triloba (L.) Dunal

\section{Annona $\mathbf{2 4 \% 9}$}

4975 glabra L.

\section{RANUNCULACEAE}

\section{Hydrastis $\mathbf{2 4 3 5}$}

4976 Canadensis L.

\section{Paeonia 2436}

4977 Brownii Dougl. 4978 Californica Nutt.

\section{CaItha $\mathbf{2 4 3}$ '}

4979 biflora DC.

4980 chelidonii Greene

498I chionophila Greene

4982 confinis Greene

4983 flabellifolia Pursh 

4984 Howellii (Huth) Greene

4985 leptosepala DC.

4986 Macounii Greene

4987 malvacea Greene

4988 natans Pall.

4989 palustris L.

4990 radicans (Forst.) A. Gray 499I rotundifolia (Huth) Greene

\section{Trollius $\mathbf{2 4 3 8}$}

4992 albiflorus (A. Gray) Rydb. 4993 laxus Salisb.

Helleborus $\mathbf{2 4 4 0}$ 4994 viridis $\mathrm{L}$.

Cammarum $\mathbf{2 4 4 1}$

$$
\text { (Eranthis) }
$$

4995 hyemale (L.) Greene

\section{Isopyrum $\mathbf{2 4 4 4}$}

4996 biternatum (Raf.) T. \& G.

4997 Hallii A. Gray

4998 occidentale H. \& A.

4999 stipitatum A. Gray

\section{Coptis $\mathbf{2 4 4 5}$}

5000 asplenifolia Salisb.

500 I biternata Huth

5002 laciniata A. Gray

5003 occidentalis (Nutt.) T. \& G.

5004 trifolia (L.) Salisb.

5005 venosa Howell

Xanthorrhiza

2446

5006 apiifolia L'Her.

\section{Actaea $\mathbf{2 4 4 8}$}

5007 alba (L.) Mill.

5008 arguta Nutt.

5009 eburnea Rydb.

5010 rubra (Ait.) Willd.

501 I dissecta Britton

5012 viridiflora Greene

\section{Cimicifuga (2448)}

50I3 Americana Miclix.

50I4 Arizonica Wats.

5015 cordifolia Pursh

5016 elata Nutt.
5017 laciniate Wats.

5018 racemosa (L.) Nutt.

5019 dissecta A. Gray

5020 rubrifolia Kearney

\section{Aquilegia $\mathbf{2 4 4 9}$}

$502 \mathrm{I}$ australis Small

5022 brevistyla Hook.

5023 caerulea James

5024 alpina Aven Nelson

5025 calcarea Jones

5026 Daileyae Eastwood

5027 glandulosa Cockerell

5028 Mancosana Eastwood

5029 pallidiflora Cockerell

5030 Canadensis L.

503I flaviflora (Tenney) Britton

5032 chrysantha A. Gray

5033 depauperata Jones

5034 desertorum (Jones) Cockerell

5035 elegantula Greene

5036 flavescens Wats.

5037 formosa Fisch.

5038 Jonesii Parry

5039 Laramiensis Aven Nelson

5040 leptocera Nutt.

504I longissima A. Gray

5042 micrantha Eastwood

5043 pubescens Coville

5044 saximontana Rydb.

5045 truncata F. \& M.

5046 vulgaris $\mathrm{L}_{4}$.

\section{Delphinium $\mathbf{2 4 5 0}$}

5047 Ajacis L.

5048 albescens Rydb.

5049 Andersonii A. Gray

5050 apiculatum Greene

505I Barbeyi Huth

5052 bicolor Nutt.

5053 montanense Rydb.

5054 Blochmanae Greene

5055 Burkei Greene

5056 Californicum 'T. \& G.

5057 camporum Greene

5058 cardinale Hook.

5059 Carolinianum Walt.

(D. azureum Michx.)

5060 Columbianum Greene 

506r Consolida L.

5062 cucullatum Aven Nelson

5063 decorum F. \& M.

5064 patens (Benth.) A. Gray

5065 depauperatum Nutt.

5066 distichum Geyer

5067 diversifolium Greene

5068 Emiliae Greene

5069 geraniifolium Rydb.

5070 Geyeri Greene

507 I glareosum Greene

5072 glaucescens Rydb.

5073 multicaule Rydb.

5074 glaucum Wats.

5075 gracilentum Greene

5076 Hanseni Greene

5077 arcuatum Greene

5078 hesperium A. Gray

5079 leucophaeum Greene

5080 macroseratilis Rydb.

508I Menziesii DC.

5082 Nelsoni Greene

5083 nudicaule T. \& G.

5084 Nuttallianum Pritzel

5085 occidentale Wats.

5086 reticulatum Aven Nelson

5087 suberosum Cockerell

5088 Oreganum Howell

5089 Parishii A. Gray

5090 Parryi A. Gray

509I pauperculum Greene

5092 Penardi Huth

5093 Purpusi T. S. Brandegee

5094 recurvatum Greene

6095 scaposum Greene

5096 scopulorum A. Gray

5097 attenuatum Jones

5098 stachydeum A. Gray

5099 simplex Dongl.

5IO0 Sonnei Greene

5IOI strictum Aven Nelson

5IO2 subalpinum (A. Gray)

Aven Nelson

5IO3 tricorne Michx.

5IO4 trolliifolium A. Gray

5I05 uliginosum Curran

5106 urceolatum Jacq.

(D. exaltatum Ait.)
5107 variegatum T. \& G.

5 I08 vimineum Don

5 ro9 virescens Nutt.

5I IO viridescens Leiberg

5III Wootoni Rydb.

\section{Aconitum $\mathbf{2 4 5 1}$}

5II 2 bulbiferum Howell

5II3 Columbianum Nutt.

5I 4 ochroleucum Aven Nelson

5 I 5 delphinifolium DC.

5II6 Noveboracense A. Gray

5II7 ramosum Aven Nelson

5II8 reclinatum A. Gray

5II9 uncinatum L.

\section{Anemone $\mathbf{2 4 5 \%}$}

$5^{120}$ Canadensis L. (A. Pennsylvanica L.)

512I Caroliniana Walt.

5122 cylindrica A. Gray

5 I 23 decapetala L.

5I24 deltoidea Hook.

5125 Drummondii Wats.

51 26 globosa Nutt.

(A. multifida of authors, not Poir.)

5I27 Grayi Britton

5128 Hudsoniana Richardson

5I29 Lyallii Britton

5I3O narcissiflora L.

5I $3^{I}$ nudicaulis A. Gray

5132 occidentalis Wats.

5I33 Oregana A. Gray

5I34 parviflora Michx.

5I35 quinquefolia $\mathrm{L}$.

(A. nemorosa of Gray's Manual, not L.)

5136 Richardsonii Hook.

5I37 riparia Fernald

5I $3^{8}$ sphenophylla Poepp.

5I39 Tetonensis Porter

5 I 40 trifolia L.

5I4I Virginiana L.

Hepatica (245\%)

5I42 acuta (Pursh) Britton

(H. acutiloba DC.)

5143 Hepatica (L.) Karst.

(H. triloba Chaix) 

Syndesmon (245\%)

(Anemonella)

$5^{1} 44$ thalictroides (L.) Hoffm.

Pulsatilla (2452)

(Anemone in part)

5145 Ludoviciana (Nutt.) Heller

(A. patens var. Nuttaliana A. Gray) 5146 multiceps Grecne

5147 occidentalis (Wats.) Freyn

\section{Clematis $\mathbf{2 4 5 3}$}

5I48 Addisonii Britton

5I49 Arizonica Heller

5I 5o Bakeri Greene

5I $5^{\text {I }}$ Baldwinii T. \& G.

5152 Bigelovii Torr.

5I53 Catesbyana Pursh

5I54 coccinea Engelm.

5I55 crispa L.

5I 56 Drummondii T. \& G.

5157 filifera Benth

$5^{1} 5^{8}$ Fremontii Wats.

5159 Gattingeri Small

5I60 glaucophylla Small

516I hirsutissima Pursh

(C. Douglasii Hook.)

5162 holosericea Pursh

$5^{\mathrm{I}} 6_{3}$ lasiantha Nutt.

5I64 ligusticifolia Nutt.

5I65 bracteata Torr.

5166 brevifolia Nutt.

5167 Californica Wats.

5168 ochroleuca Ait.

5169 ovata Pursh

5 I 70 pauciflora Nutt.

5I I r reticulata Walt.

5I 72 Scottii Porter

5I73 Simsii Sweet

(C. Pitcheri T. \& G.)

5174 Suksdorfii Robinson

5175 Viorna L.

5176 Virginiana L.

5177 Walteri Pursh

\section{Atragene (2453)}

(Clematis in part)

5r78 Americana Sims (C. verticillaris DC.)
5'79 Columbiana ('T. \& G.) Nutt. 5180 occidentalis Hornem.

5I8I tenuiloba (A. Gray) Britton

\section{Myosurus $\mathbf{2 4 5 4}$}

5182 alopecuroides Greene

$5 \mathrm{I} 83$ apetalus Gay

5 I84 cupulatus Wats.

5185 lepturus (A. Gray) Howell

5I 86 major Greene

5187 minimus L.

$5^{\mathrm{I}} 88$ sessilis Wats.

5189 tenellus Greene

5I90 amphioxys Greene

\section{Oxygraphis $\mathbf{2 4 5 5}$}

(Ranunculus in part)

519I Cymbalaria (Pursh) Prantl

\section{Beckwithia 2455a}

(Ranunculus in part)

5192 Andersonii (A. Gray) Jepson

\section{Trautvetteria $\mathbf{2 4 5 6}$}

5I93 Carolinensis (Walt.) Vail

(T. palmata F. \& M.)

5194 grandis Nutt.

\section{Ranunculus $\mathbf{2 4 5}$ '}

5195 abortivus L.

5196 eucyclus Fernald

5I97 acriformis A. Gray

5198 acris L.

5I99 adoneus A. Gray

5200 alceus Greene

5201 Alleghaniensis Britton

5202 alismellus (A. Gray) Greene

5203 alpeophilus Aven Nelson

5204 apricus Greene

5205 Arizonicus Geeene

5206 arnoglossus Greene

5207 arvensis L.

5208 Austinae Greene

5209 Bioletti Greene

52 ro Bloomeri Wats.

52II Bolanderi Greene

52 I 2 Bongardi Greene

$52 \mathrm{I} 3$ bulbosus L. 

52 I 4 Californicus Benth.

52I 5 canescens Greene

5216 crassifolius Greene

5217 cuneatus Greene

52 I 8 latilobus A. Gray

5219 calthaeflorus Greene

5220 canus Benth.

522 I Blankinshipii Robinson

5222 cardiophyllus Hook.

5223 pinetorum Greene

5224 ciliosus Howell

5225 cuneiformis Small

5226 delphinifolius Torr. (R. multifidus Pursh)

5227 digitatus Hook.

5228 Douglasii Howell (R. tenellus Nutt.)

5229 Earlei Greene

5230 Eisenii Kellogg

523 I ellipticus Greene

5232 eremogenes Greene

5233 degener Greene

5234 Eschscholtzii Schlecht.

5235 eximius Greene

5236 fascicularis Muhl.

5237 glaberrimus Hook.

5238 Gormani Greene

5239 Grayi Britton

5240 Harveyi (A. Gray) Britton

524I Hartwegi Greene

5242 hebecarpus H. \& A.

5243 hesperoxys Greene

5244 hispidus Michx.

5245 Howellii Greene

5246 hydrocharoides A. Gray

5247 hyperboreus Rottb.

5248 inamoenus Greene

5249 intermedius (Hook.) Heller

5250 Jovis Aven Nelson

525I juniperinus Jones

5252 Lapponicus L.

5253 laxicaulis Darby

5254 Lemmoni A. Gray

5255 limosus Nutt.

5256 Ludovicianus Greene

5257 Lyallii (A. Gray) Rydb. (R. Greenei Howell)

$525^{8}$ Macauleyi A. Gray
5259 Macounii Britton

(R. hispidus Hook.)

5260 macranthus Scheele

5261 maximus Greene

5262 micranthus Nutt.

5263 microlonchus Greene

5264 Mississippiensis Small

5265 Missouriensis Greene

5266 Montanensis Rydb.

5267 muricatus L.

5268 natans C. A. Meyer

5269 nivalis $\mathrm{L}$.

5270 oblongifolius E11.

527 I obtusiusculus Raf.

(R. ambigens Wats.)

5272 occidentalis Nutt.

5273 brevistylis Greene

5274 robustus A. Gray

5275 ultra-montanus Greene

5276 ocreatus Greene

5277 Oreganus (A. Gray) Howell

5278 orthorhynchus Hook.

5279 platyphyllus A. Gray

5280 ovalis Raf.

(R. rhomboideus Goldie)

528I oxynotus A. Gray

5282 Pallasii Schlecht.

5283 palmatus Ell.

5284 parviflorus L.

5285 parvulus L.

5286 pedatizdus J. E. Smith (R. affinis $\mathrm{R}$. Br.)

5287 cardiophyllus (Hook.)

Britton

5288 Pennsylvanicus L. f.

5289 Populago Greene

5290 Porteri Britton

529I Purshii Richards.

5292 pusillus Poir.

5293 Lindheimeri A. Gray

5294 pygmaeus Wahl.

5295 Rattani (A. Gray) Howell

5296 recurvatus Poir.

5297 repens $\mathrm{L}$.

5298 reptans L.

5299 rugulosus Greene

5300 Sabini R. Br.

530I samolifolius Greene 

5302 saxicola Rydb.

5303 sceleratus L.

5304 septentrionalis Poir.

5305 Marilandicus Chapm.

5306 nitidus Chapm.

5307 subaffinis (A. Gray) Rydb.

5308 subsagittatus (A. Gray) Greene

5309 Suksdorfli A. Gray

53IO sylvaticus Thuill.

53 I I trifoliatus Muhl.

53 I 2 triternatus A. Gray

5313 Turneri Greene

53I 4 Unalaschensis Bess.

53I 5 unguiculatus Greene

5316 vicinalis Greene

\section{Cyrtorhyncha (245\%)}

53I 7 neglecta Greene

53I8 ranunculina Nutt.

(Ranunculus Nuttallii A. Gray)

\section{Areteranthis (245\%)}

5319 Coolyae (Rose) Greene

\section{Ficaria (245\%)}

5320 Ficaria (L.) Karst. (Ranunculus Ficaria L.)

\section{Batrachium (245\%)}

(Ranunculus in part.)

532 I caespitosum (DC.) F. Schultz 5322 confervoides Fries

5323 divaricatum (Schranck)Wimm

5324 flaccidum (Pers.) Rupr.

5325 hederaceum (L.) S. F. Gray

5326 Lobbii (Hiern.) Howell

5327 trichophyllum (Chaix) Bossch

(Ranunculus circinatus Sibth.)

\section{Thalictrum $\mathbf{2 4 5 9}$}

5328 alpinum L.

5329 caesium Greene

5330 caulophylloides Sinall

533 I clavatum DC.

5332 coriaceum (Britton) Small

5333 debile Buckley

5334 dioicum L.

5335 Fendleri Engelm.

5336 platycarpum Trelease
5337 hesperium Greene

$533^{8}$ macrostylum (Shuttlw.)

Small \& Heller

5339 megacarpum Torr.

5340 minus Kemense Trelease

534I occidentale A. Gray

5342 polycarpum Wats.

5343 polygamum Muhl.

5344 purpurascens L.

5345 sparsiflorum Turcz.

5346 venulosum Trelease

5347 Wrightii A. Gray

\section{Adonis $\mathbf{2 4 6 0}$}

5348 annua L. (A. autumnalis L.)

\section{BERBERIDACEAE}

\section{Podophyllum $\mathbf{2 4 6 8}$}

5349 peltatum L.

\section{Jeffersonia $\mathbf{2 4 6 9}$}

5350 diphylla (L.) Pers.

\section{Diphylleia $\mathbf{2 4} \%$}

$535^{\text {I }}$ cymosa Michx.

\section{Achlys 24\%1}

5352 triphylla DC.

\section{Vancouveria $\mathbf{2 4 7}$}

5353 chrysantha Greene

5354 hexandra Morr. \& Dec.

5355 parviflora (A. Gray) Greene

\section{Caulophyllum $\mathbf{2 4 \% 4}$}

5356 thalictroides (L.) Michx.

Berberis $24 \% 5$

5357 Aquifolium Pursh (B. repens Lindl.)

$535^{8}$ Canadensis Mill.

5359 dictyota Jepson

5360 Fendleri A. Gray

536 I Fremontii Torr.

5362 haematocarpa Wooton

5363 nana Greene

5364 nervosa Pursh

5365 Nevinii A. Gray 

5366 pinnata Lag.

5367 pumila Greene

5368 Swazeyi Buckley

5369 trifoliolata Moricand

5370 vulgaris $\mathrm{L}$.

537 r Wilcoxii Kearney

MENISPERMACEAE

Menispermum $\mathbf{2 4 7 6}$

5372 Canadense L.

Cebatha 2479

(Cocculus)

5373 Carolina (L.) Britton

5374 diversifolia (DC.) Kuntze

Calycocarpum $\mathbf{2 5 0 0}$

5375 Lyoni (Pursh) Nutt.

\section{CALYCA NTHACEAE}

Butneria 2532

(Calycanthus)

5376 fertilis (Walt.) Kearney

(Calycanthus glaucus Willd.)

5377 florida (L.) Kearney

5378 occidentalis (H. \& A.) Greene

LAURACEAE

\section{Persea $255 \%$}

5379 Borbonia (L.) Spreng. ( $P$. Carolinensis Nees)

5380 humilis Nash

538r pubescens (Pursh) Sargent

(P. Carolinensis var. palustris

\section{Ocotea $256 \%$}

Chapm.)

5382 Catesbyana (Michx.) Sargent (Nectandra Willdenoviana Meisner)

\section{Umbellularia $\mathbf{2 5 6 3}$}

5383 Californica (H. \& A.) Nutt.

\section{Sassafras $\mathbf{2 5 6 8}$}

5384 Sassafras (Li.) Karst.

(S. officinale Nees \& Eiberm.)

\section{Malapoenna $\mathbf{2 5 \% 1}$}

(Litsea)

$53^{85}$ geniculata (Walt.) Coult.

\section{Benzoin 2590}

(Lindera)

5386 Benzoin (L.) Coult.

$53^{87}$ melissaefolium (Walt.) Nees

Cassytha $\mathbf{2 5 9 4}$

5388 filiformis L.

\section{PAPAVERACEAE}

Platystigma 2601

5389 Californicum (Torr. \& Frem.)

B. \& H.

5390 denticulatum Greene

539I lineare Benth.

5392 Oreganum (Nutt.) B. \& H.

\section{Platystemon 260\%}

5393 Californicus Benth.

\section{Romneya 2603}

5394 Coulteri Harvey

5395 trichocalyx Eastwood

\section{Dendromecon $\mathbf{2 6 0 4}$}

5396 flexile Greene

5397 Harfordii Kellogg

5398 rigidum Benth.

\section{Eschscholtzia $\mathbf{2 6 0 6}$}

5399 ambigua Greene

5400 caespitosa Benth.

540 I Californica Cham.

5402 cucullata Greene

5403 dolichocarpa Eastwood

5404 Douglasii Benth.

5405 glauca Greene

5406 glyptosperma Greene

5407 hypecoides Benth.

5408 Lemmoni Greene

5409 leptandra Greene

5410 maritima Greene

54I I Mexicana Greene

54 I 2 minutiflora Wats.

54I 3 Darwiniensis Jones

5414 modesta Greene

54I5 peninsularis Greene

54I6 ramosa Greene

54I 7 rhombipetala Greene 

54I8 tenuifolia Hook.

5419 tenuisecta Greene

Sanguinaria $\mathbf{2 6 0 \%}$

5420 Canadensis L.

Meconopsis

542 I heterophylla Benth.

Stylophorum 2609 5422 diphyllum (Michx.) Nutt.

\section{Chelidonium $\mathbf{2 6 1 1}$}

5423 majus L.

\section{Bocconia 2613}

5424 cordata Willd.

\section{Glaucium $\mathbf{2 6 1 4}$}

5425 Glaucium ( $\mathrm{L}_{\text {. }}$ ) Karst.

$$
\text { (G. luteum Scop.) }
$$

\section{Argemone $\mathbf{2 6 1 8}$}

5426 alba Lestib.

5427 bipinnatifida Greene

5428 corymbosa (Eastwood) Greene 5429 gracilenta Greene

5430 hispida A. Gray

543I intermedia Sweet

5432 leiocarpa Greene

5433 Mexicana L.

5434 ochroleuca Lindl.

5435 platyceras Link \& Otto

5436 sanguinea Greene

(A. Mexicana var. rosea Coult.)

5437 squarrosa Greene

\section{Papaver 2619}

5438 Argemone L.

5439 alpinum L.

5440 Californicum A. Gray

544 I crassifolium (Benth.) Greene

5442 dubium L.

5443 heterophyllum (Benth.)Greene

5444 Lemmoni Greene

5445 Macounii Greene

5446 Rhoeas L.

5447 somniferum L.

\section{Aretomecon $\mathbf{2 6 2 0}$}

5448 Californica Torr. \& Frem.
5449 humilis Coville

5450 Merriami Coville

Canbya $26 \% 1$

$545^{1}$ aurea Wats.

$545^{2}$ candida Parry

FUMARIACEAE

Bicuculla 2622

(Dicentra)

5453 Canadensis (Goldie) Millsp.

5454 chrysantha (H. \& A.) Coville

5455 Cucullaria (L.) Millsp.

5456 eximia (Ker) Millsp.

5457 formosa (DC.) Howell

$545^{8}$ ochroleuca (Engelm.) Heller

5459 pauciflora (Wats.) Coville

5460 uniflora (Kellogg) Howell

\section{Adlumia $\mathbf{2 6 2 3}$}

546I fungosa (Ait.) Greene

(A. cirrhosa Raf.)

\section{Capnoides $\mathbf{2 6 \% 4}$}

(Corydalis)

5462 aureum (Willd.) Kuntze

5463 Bidwellianum (Wats.) Greene

5464 Brandegei (Wats.) Heller

5465 Caseanum (A. Gray) Kuntze

5466 crystallinum (Engelm.)Kuntze

5467 curvisiliqum(Engelm.)Kuntze

5468 Cusickii (Wats.) Heller

5469 flavulum (Raf.) Kuntze

5470 Halei Small

547 I micranthum (Engelm.) Britton

5472 montanum (Engelm.) Britton

(C. aurea var. occidentalis Engelm.)

5473 pauciflorum (Willd.) Kuntze

5474 Scouleri (Hook.) Kuntze

5475 sempervirens (L.) Borck.

(Corydalis glauca Pursh.)

\section{Fumaria $\mathbf{2 6 \% 6}$}

5476 officinalis L.

5477 parviflora Lam.

CRUCIFERAE

Warea 2629

5478 amplexifolia (Nutt.) Small 

5479 cuneifolia Nutt. 5480 sessilifolia Nash

\section{Stanleya $\mathbf{2 6 3 0}$}

548I albescens Jones

5482 bipinnata Greene

5483 confertiflora (Robinson)

5484 elata Jones

5485 integrifolia James

5486 pinnata (Pursh) Britton

(S. pinnatifida Nutt.)

5487 tomentosa Parry

5488 viridiflora Nutt.

\section{Thelypodium $\mathbf{2 6 3 1}$}

5489 ambiguum Wats.

5490 aureum Eastwood

549I auriculatum Wats.

5492 brachycarpum Torr.

5493 Cooperi Wats.

5494 crenatum Greene

5495 elegans Jones

5496 eucosmum Robinson

5497 flavescens Wats.

5498 flexuosum Robinson

5499 Howellii Wats.

5500 Hookeri Greene

550 I integrifolium (Nutt.) Endl.

5502 gracilipes Robinson

5503 laciniatum Endl.

5504 lasiophyllum Greene

5505 inalienum Robinson

5506 Lemmoni Greene

5507 linearifolium (A. Gray) Wats.

5508 longifolium (Benth.) Wats.

5509 micranthum (A. Gray) Wats.

55 Io neglectum Jones

55 I paniculatum Aven Nelson

$55^{\text {I } 2}$ rigidum Greene

$55^{1} 3$ sagittatum (Nutt.) Heller

5514 salsugineum (Pall.) Robinson

55'5 stenopetalum Wats.

5516 torulosum Heller

$$
\text { (T. Nuttallii Wats.) }
$$

5517 Vaseyi Coult.

55I 8 Wrightii A. Gray

5519 tenellum Jones

\section{Caulanthus $263 \%$}

5520 amplexicaulis Wats.

552 i Coulteri (A. Gray) Wats.

5522 crassicaulis (Torr.) Wats.

$55^{2} 3$ glaber Jones

5524 major Jones

$55^{2} 5$ glaucus Wats.

5526 hastatus Wats.

5527 Lemmoni Wats.

5528 pilosus Wats.

5529 procerus (Brewer) Wats.

Streptanthus

$26: 33$

5530 albidus Greene

553 I argutus Greene

5532 Arizonicus Wats.

5533 asper Greene

5534 barbatus Wats.

5535 barbiger Greene

5536 Bioletti Greene

5537 bracteatus A. Gray

5538 Breweri A. Gray

5539 campestris Wats.

5540 carinatus Wright

554I cordatus Nutt.

5542 crassifolius Wats.

5543 diversifolins Wats.

5544 foliosus Greene

5545 glandulosus Hook.

5546 laevis Wats.

5547 hesperidis Jepson

5548 heterophyllus Nutt.

5549 hispidus A. Gray

5550 Howellii Wats.

555I hyacinthoides Hook.

$555^{2}$ inflatus (Wats.) Greene

5553 Lemmoni Wats.

5554 linearis Greene

5555 longirostris Wats.

5556 maculatus Nutt.

5557 Mildredae Greene

$555^{8}$ niger Greene

5559 orbiculatus Greene

5560 Parryi Greene

5561 peramaenus Greene

5562 platycarpus A. Gray

5563 polygaloides A. Gray

5564 pulchellus Greene 

5565 secundus Greene

5566 suffrutescens Greene

5567 tortuosus Kellogg

5568 versicolor Greene

5569 Wyomingensis Aven Nelson

\section{Subularia $\mathbf{2 6 4 4}$}

5570 aquatica L.

\section{Lepidium $\mathbf{2 6 4 6}$}

557 I acutidens (A. Gray) Howell 5572 alyssoides A. Gray

5573 apetalum Willd.

(L. intermedium of Gray's

Manual, not A. Gray)

5574 bipinnatifidum Desv.

5575 campestre (L.) R. Br.

5576 dictyotum A. Gray

5577 Draba L.

5578 Eastwoodiae Wooton

5579 flavum Torr.

5580 Fremontii Wats.

558i Groenlandicum Hornem.

$55^{82}$ Idahoense Heller

$55^{8} 3$ integrifolium Nutt.

5584 Jaredi Brandegee.

$55^{85}$ lasiocarpum Nutt.

$55^{86}$ tenuipes Wats.

$55^{8} 7$ latipes Hook.

$55^{88}$ medium Greene

$$
\text { (L. intermedium A. Gray) }
$$

$55^{89}$ pubescens (Greene)

5590 Menziesii DC.

559I montanum Nutt.

5592 nanum Wats.

5593 nitidum Nutt.

5594 insigne Greene

5595 occidentale Howell

5596 Oreganum Howell

5597 strictum Wats.

5598 oxycarpum T. \& G.

5599 ramosissimum Aven Nelson

5600 ramosum Aven Nelson

560 r reticulatum Howell

5602 ruderale $\mathrm{L}$.

5603 sativum L.

5604 scopulorum Jones

5605 spathulatum Robinson
5606 simile Heller

5607 sordidum A. Gray

5608 strictum Rattan

5609 Thurberi Wooton

56 ro Utahense Jones

56I I Virginicum L.

\section{Coronopus $\mathbf{2 6 4}$}

(Senebiera)

56I 2 Coronopus (L.) Karst.

5613 didymus (L.) J. E. Smith

\section{Thlaspi $\mathbf{2 6 6 5}$}

56I4 alpestre L.

56 I 5 glaucum Nelson

5616 arvense $L$.

56I7 Californicum Wats.

5618 parviflorum Aven Nelson

5619 perfoliatum L.

\section{Cochlearia $\mathbf{2 6 6 9}$}

5620 Anglica L.

562 I Danica L.

5622 fenestrata $\mathrm{R}$. Br.

5623 Groenlandica L.

5624 oblongifolia Lange

5625 officinalis L.

5626 tridactylites Banks

\section{Eutrema $\mathbf{2 6 \% 5}$}

5627 arenicola Richards.

5628 Edwardsii R. Br.

5629 Eschscholtzianum (Andrz.)

Robinson

5630 Labradoricum Turcz.

\section{Alliaria $26 \% 6$}

563I Alliaria (L.) Britton

(Sisymbrium Alliaria Scop.)

Sisymbrium 2679

5632 acutangulare DC.

5633 altissimum L.

5634 diffusum A. Gray

5635 humile Meyer

5636 Irio L.

5637 officinale (L.) Scop.

5638 pauciflorum Nutt.

5639 Vaseyi Wats.

5640 virgatum Nutt. 

Schoenocrambe $26 \% 9$ a (Sisymbrium in part) 564 I linifolia (Nutt.) Greene 5642 pinnata Greene 5643 pygmaea (Nutt.) Greene

\section{Cakile $268 \%$}

5644 edentula (Bigel.) Hook. (C. Americana Nutt.)

5645 fusiformis Greene 5646 maritim aequalis Chapm.

\section{Myagrum}

5647 perfoliatum L.

\section{Simapis $270 \%$}

5648 alba L.

\section{Diplotaxis 2708}

5649 muralis (L.) DC.

5650 tenuifolia (L.) DC.

\section{Brassica $\%$ \%11}

$5^{6} 5 \mathrm{I}$ arvensis (L.) B. S. P. (B. Sinapistrum Boiss.)

5652 campestris L.

5653 juncea (L.) Cosson

5654 Napus L.

5655 nigra (L.) Koch

5656 rapa $L$.

Raphanus 2\%1\%

5657 Raphanistrum L.

$565^{8}$ sativus $\mathrm{L}$.

\section{Rapistrum}

5659 rugosum (L.) All.

\section{Barbarea 27\%3}

5660 Americana Rydb.

5661 Barbarea (L.) MacM. (B. vulgaris R. Br.)

5662 praecox (J. E. Smith) R. Br. 5663 stricta Andrz.

\section{Dryopetalon 27\%6}

5664 runcinatum A. Gray

Roripa 27\%

(Nasturtium)

5665 alpina (Wats.) Rydb.
5666 Americana (A. Gray) Britton (N. lacustre A. Gray)

5667 Armoracia (L.) A. S. Hitchc. 5668 calycina (Engelm.) Rydb.

5669 Columbiae (Suksdorf) Howell

5670 curvipes Greene

567 I curvisiliqua (Hook.) Bessey

5672 dictyota Greene

5673 hispida (Desv.) Britton

5674 lyrata (Nutt.) Greene

5675 multicaulis Greene

5676 Nasturtium (L.) Rusby (N. officinale R. Br.)

5677 Nuttallii (Wats.) Rydb.

5678 obtusa (Nutt.) Britton

5679 occidentalis Greene

5680 Pacifica Howell

568I palustris (L.) Bess.

5682 polymorpha. (Nutt.) Howell

5683 sessiliflora (Nutt.) A. S. Hitchc.

5684 sinuata (Nutt.) A. S. Hitchc.

5685 pubescens (Wats.) Howell

5686 sphaerocarpa (A. Gray) Britton

5687 sylvestris (L.) Bessey

5688 tenerrima Greene

5689 trachycarpa (A. Gray) Greene

5690 Walteri (Ell.) Mohr

(N. tanacetifolium H. \& A.)

\section{Cardamine $27 \% 8$}

5691 angulata Hook.

5692 arenicola Britton

5693 bellidifolia L.

5694 pachyphylla Coville \&

5695 Breweri Wats.

Leiberg

5696 bulbosa (Schreb.) B. S. P.

(C. rhomboidea Pers.)

5697 callosicrenata Piper

5698 Clematitis Shuttlw.

5699 cordifolia A. Gray

5700 curvisiliqua Shuttlw.

570 I flexuosa With.

$57 \mathrm{O} 2$ fulcrata Greene

5703 Gambelli Wats.

5704 gemmata Greene

5705 hirsuta L.

5706 integrifolia Greene 

5707 Leibergii Holzinger

5708 Lyallii Wats.

5709 neglecta Greene

57 ro Nuttallii Greene

57 I I occidentalis (Wats.) Howell

5712 oligosperma Nutt.

57I3 parviflora L.

57I4 paucisecta Benth.

57 I5 Pennsylvanica Muhl.

5716 pratensis $\mathrm{L}$.

5717 angustifolia Hook.

57I 8 purpurea ('Torr.) Britton

5719 rotundifolia Michx.

5720 umbellata Greene

5721 unijuga Rydb.

5722 vallicola Greene

\section{Sibara 2\%88}

5723 filifolia Greene

\section{Dentaria $2 \% 29$}

5724 Caltfornica Nutt.

5725 cardiophylla(Greene)Robinson

5726 cuneata Greene

5727 diphylla Michx.

5728 gemmata Wats.

5729 heterophylla Nutt.

5730 laciniata Muhl.

573 I macrocarpa Nutt.

5732 maxima Nutt.

5733 multifida Muhl.

5734 pachystigma Wats.

5735 pulcherrima Greene

5736 quercetorum (Howell) Greene

5737 sinuata Greene

5738 tenella Pursh

\section{Lunaria 2731}

5739 annua L.

5740 rediviva $\mathrm{L}$.

\section{Selenia 273\%}

5741 aurea Nutt.

5742 dissecta T. \& G.

\section{Leavenworthia $\quad 27: 33$}

5743 aurea Torr.

5744 stylosa A. Gray

5745 torulosa A. Gray

5746 uniflora (Michx.) Britton

\section{Platyspermum 2734}

5747 scapigerum Hook.

\section{Tropidocarpum $2 \% 36$}

5748 capparideum Greene

5749 dubium Davidson

$575^{\circ}$ gracile Hook.

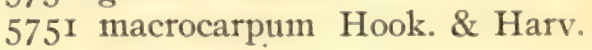

\section{Synthlipsis $2 \% 41$}

5752 Berlandieri A. Gray

5753 hispida Wats.

5754 Greggii A. Gray.

\section{Dithyrea}

5755 Californica Harv.

$575^{6}$ maritima Davidson

5757 Wislizeni Engelm.

\section{Lyrocarpa $\mathbf{2 7 4 3}$}

$575^{8}$ Coulteri Hook. \& Harv.

5759 Palmeri Wats.

\section{Physaria 2\%44}

5760 didymocarpa (Hook.) A. Gray

576r Geyeri (Hook.) A. Gray

5762 Newberryi A. Gray

5763 Oregana Wats.

\section{Lesquerella $\mathbf{2 7 4 5}$}

5764 alpina (Nutt.) Wats.

5765 angustifolia (Nutt.) Wats.

5766 arctica (DC.) Wats.

5767 Purshii Wats.

5768 argentea (Pursh) MacM.

(L. Ludoviciana Wats.)

5769 argyraea (A. Gray) Wats.

5770 Arizonica Wats.

577 I aurea Wooton

5772 auriculata (Engelm. \& Gray)

Wats.

5773 cinerea Wats.

5774 condensata Aven Nelson

5775 curvipes Aven Nelson

5776 densiflora (A. Gray) Wats.

5777 Douglasii Wats.

5778 Engelmanni (A. Gray) Wats.

5779 Fendleri (A. Gray) Wats.

5780 globosa (Desv.) Wats.

578r Gordoni (A. Gray) Wats. 

5781 gracilis (Hook.) Wats.

5782 sessilis Wats.

5783 grandiflora (Hook.) Wats.

5784 intermedia (Wats.) Heller

5785 Kingii Wats.

5786 lasiocarpa (Hook.) Wats.

5787 Lescurii (A. Gray) Wats.

5788 Lindheimeri (A. Gray) Wats.

5789 montana (A. Gray) Wats.

5790 Nuttallii ('T. \& G.) Wats.

5791 occidentalis Wats.

5792 ovalifolia Rydb.

5793 pallida (Nutt.) Wats.

5794 Palmeri Wats.

5795 prostrata Aven Nelson

5796 purpurea (A. Gray) Wats.

5797 recurvata (Engelm.) Wats.

5798 repanda (Nutt.) Wats.

5799 rosulata Aven Nelson

5800 spathulata Rydberg

580 I Wardii Wats.

\section{Hutchinsia 2\%4\%}

5802 procumbens (L.) Desv.

(Capsella elliptica C. A. Meyer)

\section{Bursa 2748}
(Capsella)

5803 Bursa-Pastoris (L.) Britton

\section{Camelima $\boldsymbol{2 \% 4 9}$}

5804 microcarpa Andrz.

(C. sylvestris Wallr.)

5805 sativa (L.) Crantz

\section{Neslia $2 \% 50$}

5806 paniculata (L.) Desv.

\section{Draba $2 \% 51$}

$5^{807}$ alpina L.

5808 andina (Nutt.) Aven Nelson

5809 arabisans Michx.

5810 arctica Vahl.

58I I asprella Greene

5812 aurea Vahl.

5813 aureola Wats.

5814 borealis DC.

$5^{81} 5$ brachycarpa Nutt.

$58 \mathrm{I} 6$ Breweri Wats.
$5^{8 \mathrm{I}} 7$ Caroliniana Walt.

5818 chrysantha Wats.

5819 corrugata Wats.

$5^{820}$ corymbosa R. Br.

5821 crassa Rydb.

5822 crassifolia Graham

5823 Crockeri Lemmon

5824 Columbiae Howell

$5^{825}$ cuneifolia Nutt.

5826 integrifolia Wats.

5827 deflexa Greene

5828 densifolia Nutt.

(D. glacialis var. pectinata Wats.)

$5^{829}$ Douglasii A. Gray

5830 eurycarpa A. Gray

583 I Fladnizensis Wulf.

5832 glabella Adams

5833 glacialis Adams

5834 Helleriana Greene

5835 hirta L.

$5^{8} 36$ Howellii Wats.

5837 incana L.

$5^{8} 3^{8}$ laevipes DC.

$5^{8} 39$ Lemmoni Wats.

5840 lonchocarpa Rydb.

(D. nivalis var. elongata Wats.)

584 I lutea Gilib.

5842 luteola Greene

5843 micrantha Nutt.

5844 Mogollonica Greene

5845 montana Wats.

5846 nemorosa L.

5847 Neo-Mexicana Greene

5848 robusta Heller

5849 nivalis Lilj.

5850 oligosperma Hook.

585 I pallida Heller

$585^{2}$ patens Heller

5853 petrophila Greene

$5^{8} 54$ pinetorum Greene

$5^{8} 55$ platycarpa T. \& G.

5856 ramosissima Desv.

5857 saximontana Aven Nelson

$5^{8} 5^{8}$ sessilis Wats.

5859 Sonorae Greene

5860 spectabilis Greene

586 I stenoloba Ledeb.

5862 streptocarpa A. Gray 

5863 subsessilis Wats.

5864 surculifera Aven Nelson

5865 ventosa A. Gray

5866 verna $\mathrm{L}$.

\section{Heterodraba 2751a}

5867 unilateralis (Jones) Greene

(Draba unilateralis Jones)

\section{Nesodraba $\mathbf{2 7 5 1 b}$}

5868 grandis (Langsd.) Greene

(Draba grandis Langsd.)

5869 megalocarpa Greene

5870 siliquosa (Schlecht.) Greene

(Cochlearia siliquosa Schlecht.)

[This genus is the Draba

hyperborea of the Synoptical Flora. ]

\section{Athysanus $\mathbf{2 7 5 4}$}

587 I pusillus (Hook.) Greene 5872 glabrior Wats.

\section{Thysanocarpus $\mathbf{2 7 5 5}$}

$5^{873}$ amplectens Greene

5874 conchuliferus Greene

5875 planiusculus Robinson

5876 crenatus Nutt.

5877 curvipes Hook.

5878 involutus Greene

5879 elegans F. \& M.

5880 emarginatus Greene

588 I erectus Wats.

5882 hirtellus Greene

5883 laciniatus Nutt.

5884 pulchellus F. \& M.

5885 radians Benth.

5886 ramosus Greene

\section{Smelowskia 2758}

5887 calycina (Desv.) C. A. Meyer 5888 Fremontii Wats. 5889 ovalis Jones

\section{Sophia 2759}

5890 andrenarum Cockerell

5891 osmiarum Cockerell

5892 filipes (A. Gray) Heller

5893 halictorum Cockerell

5894 Hartwegiana (Fourn.) Greene
5895 incisa (Engelm.) Greene

5896 intermedia Rydb.

5897 ochroleuca Wooton

5898 pinnata (Walt.) Howell

(Sisymbrium canescens Nutt.)

5999 Sonnei (Robinson) Greene

5900 Sophia (L.) Britton

590 I sophioides (Fisch.) Heller

\section{Stenophragma 2761}

5902 Thaliana (L.) Celak. (Sisymbrium Thalianum Gay)

\section{Arabis $2 \% 63$}

5903 alpina L.

5904 arcuata A. Gray

5905 longipes Wats.

5906 subvillosa Wats.

5907 atrorubens Suksdorf

5908 Beckwithii Wats.

5909 blepharophylla H. \& A.

59 IO Bolanderi Wats.

59II Bourgovii Rydb. (A. patula Graham)

5912 brachycarpa (T. \& G.) Britton (L. confinis Wats.)

5913 Brebneriana Aven Nelson

5914 Brentilii Lange

5915 Breweri Wats.

5916 Canadensis L.

5917 canescens Nutt.

59 I 8 stylosa Wats.

5919 Columbiana Macoun

5920 Crandallii Robinson

592 I Cusickii Wats.

5922 dispar Jones

5923 drepanoloba Greene

5924 Drummondii A. Gray

5925 exilis Aven Nelson

5926 Fendleri (Wats.) Greene

5927 filifolia Greene

5928 furcata Wats.

5929 glabra (L.) Bernh.

$$
\text { (A. pcrfoliata Lam.) }
$$

5930 glaucovalvula Jones

5931 hirsuta (L.) Scop.

5932 glabrata T. \& G.

5933 Holboelii Hornem.

5934 Hookeri Lange 

5935 Howellii Wats.

5936 Koehleri Howell

5937 humifusa (G. Vahl.) Wats.

5938 pubescens Wats.

5939 laevigata (Muhl.) Poir.

5940 Burkei Porter

594I laciniata T. \& G.

5942 Lemmoni Wats.

5943 lignifera Aven Nelson

5944 Lyallii Wats.

5945 lyrata L.

5946 occidentalis Wats.

5947 Macounii Wats.

5948 microphylla Nutt.

5949 Nuttallii Robinson (A. spathulata Nutt.)

5950 Parishii Wats.

595I patens Sullivant

5952 perennans Wats.

5953 petiolaris A. Gray

5954 platysperma A. Gray

5955 praealta Greene

5956 puberula Nutt.

5957 pulchra Jones

$595^{8}$ gracilis Jones.

5959 purpurascens Howell

5960 repanda Wats.

5961 retrofracta Graham

5962 rhodantha Greene

5963 secunda Howell

5964 sparsiflora Nutt.

5965 stricta Huds.

5966 Suksdorfii Howell

5967 subpinnatifida Wats.

5968 suffrutescens Wats.

5969 Virginica (L.) Trelease

(A. Ludoviciana C. A. Meyer)

\section{Iodanthus (2763)}

5970 dentatus ('T. \& G.) Greene (Arabis dentata T. \& G.)

597 I pinnatifidus (Michx.) Steud.

\section{Parrasia 2765}

(Greggia)

5972 camporum (A. Gray) Greene 5973 linearifolia (Wats.) Greene
Cheiranthus $2 \% 66$

(Erysimum)

5974 angustatus Greene

5975 arenicola (Wats.) Greene

5976 aridus Aven Nelson

5977 argillosus Greene

5978 Arkansanus (Nutt.) Greene

5979 asper Nutt.

5980 asperrimus (Nutt.) Greene

598I Californicus Greene

5982 capitatus Dougl.

(E. grandiflorum Nutt.)

5983 cheiranthoides (L.) Heller

5984 elatus (Nutt.) Greene

5985 inconspicuus (Wats.) Greene (E. parviflorum Nutt.)

5986 insularis Greene

5987 nivalis Greene

5988 amoenus Greene

5989 occidentalis Wats.

5990 perennis (Coville) Greene

5991 pygmaeus Adams

5992 syrticola (Sheldon) Greene

5993 Wheeleri (Rothrock) Greene

\section{Alyssum 2768}

5994 alyssoides (L.) Gouan (A. calycinum L.)

5995 Americanum Greene

\section{Konig ( $2 \% 68)$}

5996 maritima (L.) R. Br. (Alyssum maritimum L.)

\section{Berteroa 27\%}

5997 incana (L.) DC.

\section{Braya 27\%9}

5998 pilosa Hook.

5999 purpurascens Bunge

\section{Hesperis 2799}

6000 matronalis $\mathrm{L}$.

\section{Parrya 2810}

6oor arctica $\mathrm{R}$. Br.

6002 macrocarpa R. Br. 

Phoenicaulis 2811

6003 Menziesii (Hook.) Greene 6004 lanuginosa (Wats.) Heller

\section{Conringia $\mathbf{2 8 1 \%}$}

6005 orientalis (L.) Dumort

\section{Stanfordia $\mathbf{2 8 3 0}$}

6006 Californica Wats.

\section{CAPPARIDACEAE}

\section{Cleome $\mathbf{2 8 3 6}$}

6007 inornata Greene

6008 lutea Hook.

6009 pentaphylla L.

6010 platycarpa Torr.

60I I serrulata Pursh

$$
\text { (C. integrifolia T. \& G.) }
$$

6012 Sonorae A. Gray.

6or 3 sparsiflora Wats.

6or4 spinosa $\mathrm{L}$.

\section{Cleomella 283'}

6015 angustifolia Torr.

6016 brevipes Wats.

6017 gracilis T. S. Brandegee

6018 longipes Torr.

6019 grandiflora Wats.

6020 obtusifolia Torr. \& Frem.

6021 oocarpa A. Gray

6022 Palmerana Jones

6023 parviflora A. Gray

6024 plocasperma Wats.

\section{Wislizenia 2838}

6025 Palmeri A. Gray

6026 refracta Engelm.

\section{Oxystylis (2838)}

6027 lutea Torr. \& Frem.

\section{Isomeris $\quad 2839$}

6028 arborea Nutt.

6029 globosa Coville

Cristatella $\mathbf{2 8 4 \%}$

6030 erosa Nutt.

603I Janesii T. \& G.
Polanisia 2843

6032 graveolens Raf.

6033 tenuifolia T. \& G.

6034 trachysperma 'T. \& G.

6035 uniglandulosa DC.

\section{Atamisquea 285:3}

6036 emarginata Miers

Capparis $\mathbf{2 8 5 4}$

6037 cynophallophora Jacq.

6038 Jamaicensis Jacq.

\section{RESEDACEAE}

\section{Reseda $28 \% 3$}

6039 alba I.

6040 lutea L.

604I luteola L.

Oligomeris $28 \% 5$

6042 glaucescens Camb.

Dipetalia (2875)

6043 subulata (Webb. \& Berth.)

Kuntze

\section{SARRACENIACEAE}

\section{Sarracenia $28 \% 8$}

6044 Catesbaei Ell.

6045 Drummondii Croom

6046 flava L.

6047 psittacina Michx.

6048 purpurea L.

6049 heterophylla (Eaton) Torr.

6050 rubra Walt.

605 I variolaris Michx.

\section{Chrysamphora 2879}

(Darlingtonia)

6052 Californica (Torr.) Greene

\section{DROSERACEAE}

\section{Dionaea 2880}

6053 muscipula Ell.

\section{Drosera 288:3}

6054 brevifolia Pursh

6055 capillaris Poir. 

6056 filiformis Raf.

6057 intermedia Hayne

6058 linearis Goldie

6059 longifolia L.

6060 rotundifolia L.

\section{PODOSTEMACEAE}

\section{Podostemon $\mathbf{2 9 0 \%}$}

606 I ceratophyllum Michx.

6062 abrotanoides Wedd.

\section{CRASSULACEAE}

\section{Sedum 2906}

6063 acre L.

6064 annuum Wats.

6065 ciliosum Howell

6066 Congdoni Eastwood

6067 debile Wats.

6068 divaricatum Wats.

6069 divergens Wats.

6070 Douglasii Hook.

6071 Liebmannianum Hemsl.

6072 Nevii A. Gray

6073 obtusatum A. Gray

6074 Oreganum Nutt.

6075 pulchellum Michx.

6076 pumilum Benth.

6077 pusillum Michx.

6078 radiatum Wats.

6079 reflexum L.

6080 rhodanthum A. Gray

608 r roseum (L.) Scop.

(S. Rhodiola DC.)

6082 spathulifolium Hook.

6083 stelliforme Wats.

6084 stenopetalum .Pursh

6085 rubrolineatum Cockerell

6086 telephioides Michx.

6087 Telephium L.

6088 ternatum Michx.

6089 Texanum J. G. Smith

6090 Torreyi Don

6o9r uniflorum Howell

6092 variegatum Wats.

6093 villosum L.

6094 Wrightii A. Gray

Sempervium 290\%

6095 tectorum L.

\section{Cotyledon $\mathbf{2 9 0 9}$}

6096 caespitosa Haw.

6097 edulis (Nutt.) Brewer

6098 farinosa (Lindl.) Baker

6099 lanceolata (Nutt.) B. \& H.

6 roo laxa (Lindl.) B. \& H.

6ror linearis Greene

$6 \mathrm{IO} 2$ lingula Wats.

6ro3 Nevadensis Wats.

6104 Oregonensis Wats.

6ro5 Palmeri Wats.

6106 Peacockii Baker

6107 pulverulenta (Nutt.) Baker

6ro8 Rusbyi Greene

6rog saxosum Jones

6r Io strictiflora (A. Gray) Baker

6I I I viscida Wats.

\section{Bryophyllum 2910}

6 II2 calycinum Salisb.

Tillaea (2912)

6I I 3 angustifolla Nutt.

6II4 aquatica L.

(T. simplex Nutt.)

6II5 Bolanderi (Wats.) Greene

6II6 Drummondii T. \& G.

6II7 minima Miers

6II 8 subsimplex Wats.

\section{Diamorpha 2916}

6 Ir9 pusilla Nutt.

Penthorum 291\%

6 I 20 sedoides L.

SAXIFRAGACEAE

\section{Astilbe $\mathbf{2 9 2 0}$}

6I2I biternata (Vent.) Britton

(A. decandra Don)

6122 crenatiloba.(Britton)

Wheelock

\section{Leptarrhena $29 \%$}

6r 23 amplexifolia (Sternb.) Ser.

Bolandra $29 \% 6$

6r 24 Californica A. Gray

6r 25 Oregana Wats. 



\section{Therofon 292\%}

(Boykinia)

6r 26 aconitifolium (Nutt.) Millsp. 6 r27 elatum (Nutt.) Greene

6128 heucheraeforme Rydb.

6r29 Jamesii (Torr.) Wheelock (Saxifraga Jamesii Torr.)

6r 30 majus (A. Gray) Wheelock

6r 3 I Purpusi ('T. S. Brandegee) Heller

6I32 Richardsonii(Hook.)Wheelock $6 \mathrm{r} 33$ rotundifolium (Parry)

\section{Sullivantia 2928}

6r34 Hapemani (Coult. \& Fisher)

6r35 Oregana Wats.

6r36 Sullivantii ('T. \& G.) Britton (S. Ohionis T. \& G.)

\section{Hemieva $\mathbf{2 9 \% 9}$}

(Suksdorfia)

6I 37 ranunculifolia (Hook.) Raf.

6r $3^{8}$ violacea (A. Gray) Wheelock

\section{Saxifraga 2931}

6r39 adscendens L.

6I4O aestivalis Fisch.

6I4I aizoides $\mathrm{L}$.

6I42 Aizoon Jacq.

6I43 apetala Piper

6I44 aprica Greene

6r45 austromontana Wiegand

6r46 Bongardi Presl

6I 47 bronchialis L.

6r 48 bryophora A. Gray

6r 49 caespitosa L.

6r 50 Groenlandica Wahl.

6I 5I Sternbergii Regel

6r 52 uniflora Hook.

6I 53 Californica Greene

6I 54 Careyana A. Gray

6r 55 cernua L.

6r 56 cherlerioides D. Don

6r 57 chrysantha A. Gray

6i 58 claytoniaefolia Canby

6I 59 Columbiana Piper

6160 comosa (Poir.) Britton

(S. foliosa R. Br.) 6r6 I debilis Engelm.

6162 eriophora Wats.

6r63 Eschscholtzii Sternb.

6I64 exilis Stephen

6I65 fallax Greene

6r66 ferruginea Graham

6I67 flagellaris Willd.

6r68 Forbesii Vasey

6r69 fragosa Suksdorf

6I 70 Geum L.

6I 7 I Grayana Britton

(S. Caroliniana A. Gray)

6 I 72 hieracifolia Waldst. \& Kit.

6I73 Hirculus L.

6I 74 Howellii Greene

6I75 Idahoensis Piper

6I 76 integrifolia Hook.

6r77 ledifolia Greene

6r 78 Lyallii Engler

6r79 laxa Engler

6r8o Marshallii Greene

6I8I Mertensiana Bong.

6r82 Michauxii Britton

(S. leucanthemifolia Michx.)

6I83 micranthidifolia (Haw.) B.S.P.

(S. erosa Pursh)

6r84 Montanensis Small

6 I85 Napensis Small

6I86 nidifica Greene

6 I87 nivalis $\mathrm{L}$.

6r 88 tenuis Wahl.

6189 Nootkana Moc.

6190 nudicaulis Don

6I 9I Nuttallii Small

6I92 occidentalis Wats.

6r93 oppositifolia L.

6r94 Oregana Howell

6I95 parvifolia Greene

6rg6 peltata Torr.

6r97 Pennsylvanica L.

6r98 plantaginea Small

6I99 punctata L.

6200 acutidentata Engler

6201 nana A. Gray

6202 radulina Greene

6203 ranunculifolia Hook.

6204 reflexa Hook.

6205 rhomboidea Greene 

6206 rivularis $\mathrm{I}$.

6207 hyperborea Hook. 6208 Lamentina Engler

6209 Rydbergii Small

6210 saximontana Elias Nelson

62 II serpyllifolia Pursh

62 I 2 Sierrae (Coville) Small

6213 sileniflora Sternb.

6214 stellaris L.

6215 comosa Poir.

62 I6 subapetala Elias Nelson

6217 Tennesseensis Small

6218 Tolmiei T. \& G.

6219 tricuspidata Retz.

6220 micrantha Sternb.

6221 Unalaschensis Sternb.

6222 Van-Bruntiae Small

6223 Virginiensis Michx.

\section{Saxifragopsis 2931 a}

6224 fragarioides (Greene) Small

\section{Jepsonia $2931 \mathrm{~b}$}

(Saxifraga in part)

6225 malvaefolia (Greene) Small 6226 Parryi ('Torr.) Small

\section{Peltiphyllum 293\%}

6227 peltatum (Torr.) Engelin.

\section{Tiarella $\mathbf{2 9 3 5}$}

6228 cordifolia L.

6229 laciniata Hook.

6230 trifoliata L.

6231 unifoliata Hook.

\section{Henchera $\mathbf{2 9 3}$}

6232 Americana L.

6233 bracteata (Torr.) Seringe

6234 ciliata Rydb.

6235 cuneata Howell

6236 cylindrica Dougl.

6237 alpina Wats.

$623^{8}$ tenuifolia Wheelock

6239 glabella T. \& G.

6240 glabra Willd.

624I grossulariifolia Rydb.

6242 Hallii A. Gray

6243 hispida Pursh

6244 hirsuticaulis Wheelock
6245 macrorhiza Small

6246 maxima Greene

6247 micrantha Dougl.

6248 Nova-Mexicana Wheelock

6249 ovalifolia Nutt.

6250 parvifolia Nutt.

625 I pilosissima F. \& M.

6252 pubescens Pursh

6253 racemosa Wats.

6254 rubescens Torr.

6255 nana A. Gray

6256 Pregonensis Wheelock

6257 Rugelii Shuttlw.

6258 sanguinea Engelm.

6259 villosa Michx.

\section{Leptaxis $\mathbf{2 9 3 8}$}

(Tolmiea)

6260 Menziesii (Pursh) Raf.

\section{Tellima 2939}

626I grandiflora (Pursh) Dougl.

6262 odorata Howell

6263 racemosa (Wats.) Greene

\section{Lithophragma (2939)}

(Tellima in part)

6264 affinis A. Gray

6265 Bolanderi A. Gray

6266 campanulata Howell

6267 Cymbalaria T. \& G.

6268 glabra Nutt.

6269 heterophylla T. \& G.

6270 parviflora (Hook.) Nutt.

627 I rupicola Greene

6272 scabrella Greene

6273 tenella Nutt.

6274 tripartita Greene

6275 Williamsii (D.C.Eaton) Greene

(Heuchera Williamsii D. C. Eaton)

\section{Mitella $\mathbf{2 9 4 0}$}

6276 Breweri A. Gray

6277 diphylla L.

6278 diversifolia Greene

6279 nuda L.

6280 ovalis Greene

628I pentandra Hook. 

6282 trifida Graham

6283 violacea $R y d b$.

Mitellastra 2940a

6284 caulescens (Nutt.) Howell (Mitella caulescens Nutt.)

\section{Chrysosplenium $\mathbf{2 9 4 1}$}

6285 alternifolinm L.

6286 Americanum Schwein

6287 Beringianum Rose

6288 Scouleri (Hook.) Rose

(C. glechomaefolia Nutt.)

6289 tetrandrum $T h$. Fr.

Lepuropetalon $\mathbf{2 9 4} \%$ 6290 spathulatum (Muh1.) E11.

\section{Parnassiaceae}

\section{Parnassia $\mathbf{2 9 4 5}$}

6291 asarifolia Vent.

6292 Californica (A. Gray) Greene

6293 Caroliniana Michx.

6294 fimbriata Banks

6295 grandifolia DC.

6296 Kotzebuei C. \& S.

6297 palustris L.

6298 parviflora DC.

\section{HYDRANGEACEAE}

Carpenteria $\mathbf{2 9 4 9}$

6299 Californica Torr.

\section{Philadelphus $\mathbf{2 9 5 0}$}

6300 argyrocalyx Wooton

6301 coronarius $\mathrm{L}$.

$63 \mathrm{O} 2$ Gordonianus Lindl.

6303 grandiflorus Willd.

6304 floribundus 'T. \& G.

6305 laxus T. \& G.

6306 hirsutus Schrad.

6307 inodorus L.

6308 latifolius Schrad.

6309 Lewisii Pursh

63Io Californicus A. Gray

63II microphyllus A. Gray

63 I2 occidentalis Aven Nelson

6313 serpyllifolius A. Gray
Edwinia 2951

(Jamesia)

63 I4 Americana ('T. \& G.) Heller

\section{Fendlera $\mathbf{2 9 5 \%}$}

63I5 rupicola Engelm. \& Gray

63 I6 Wrightii (Engelm. \& Gray)

Heller

\section{Fendlerella 295\%a}

63I7 Utahensis (Wats.) Heller (Whipplea Utahensis Wats.)

\section{Whipplea $\mathbf{2 9 5 4}$}

631 8 modesta Torr.

\section{Hydrangea 2958}

6319 arborescens L.

6320 cordata T. \& G.

6321 Kanawahana Millsp.

6322 cinerea Small

6323 quercifolia Bartr.

6324 radiata Walt.

\section{Decumaria $\mathbf{2 9 6 3}$}

6325 barbara L.

\section{Itea $29 \%$}

6326 Virginica L.

\section{GROSSULARIACEAE}

\section{Ribes $\mathbf{2 9 8}$ \%}

6327 amarum McClatchie

6328 amictum Greene

6329 aridum Greene

6330 aureum Pursh

633I chrysococcum Rydb.

6332 binominatum Heller

(Ribes montanum Howell)

6533 brachyanthum (A. Gray) Card

6334 bracteosum Dougl.

6335 Californicum H. \& A.

9336 cereum Dougl.

6337 pedicellare A. Gray

6338 ciliosum Howell

6339 cognatum Greene

6340 cruentum Greene

634 I curvatum Small

6342 Cynosbati L. 

6343 divaricatum Dougl.

6344 echinatum Lindl.

6345 erythrocarpum Coville \&

Leiberg

6346 floridum L'Her

6347 glutinosum Benth.

6348 melanocarpum Greene

6349 gracile Michx.

6350 hesperium McClatchie

635 I Howellii Greene

6352 Hudsonianum Richards

6353 inerme Rydb.

6354 irriguum Dougl.

6355 lacustre (Pers.) Poir.

6356 lasianthum Greene

6357 lasiococcus A. Gray

$635^{8}$ laxiflorus Pursh

6359 leptanthum A. Gray

6360 leucoderme Heller

636I Lobbii A. Gray

6362 malvaceum Smith

6363 Marshallii Greene

6364 Menziesii Pursh

6365 Missouriense Nutt.

6366 Mogollonicum Greene

6367 molle (A. Gray) Howell

6368 montigenum McClatchie

6369 Nevadense Kellogg

6370 nigrum L.

637 I niveum Lindl.

6372 oxyacanthoides L.

6373 Palmeri Vasey \& Rose

6374 parvulum (A. Gray) Rydb.

6375 pinetorum Greene

6376 prostratum L'Her.

6377 quercetorum Greene

6378 resinosum Pursh

6379 Roezli Regel

6380 rotundifolium Michx.

638 I rubrum $\mathrm{L}$.

6382 Rusbyi Greene

6383 sanguineum Pursh

6384 variegatum Wats.

6385 saxosum Lindl.

6386 setosum Lindl.

6387 Spaethianum Koehne

6388 speciosum Pursh

6389 subvestitum H. \& A.
6390 tenuiflorum Lindl.

639 I tortuosum Benth.

6392 Uva-crispa I.

6393 velutinum Greene

6394 viburnifoliuu A. Gray

6395 Victoris Greene

6396 viscosissimum Pursh

6397 Watsonianum Koehne (R. ambiguum Wats.)

6398 Wilsonianum Greene

6399 Wolfii Rothrock

HAMAMELIDACEAE

Liquidambar $\mathbf{3 0 2 0}$

6400 Styraciflua L.

Fothergilla $\mathbf{3 0 \% 4}$

640 I Carolina (I.) Britton

(F. Gardeni Murr.)

6402 monticola Ashe

Hamamelis 3031

6403 Virginiana L.

PLATANACEAE

\section{Platanus 3048}

6404 occidentalis L.

6405 racemosa Nutt.

6406 Wrightii Wats.

\section{CROSSOSOMATACEAE}

\section{Crossosoma 3048}

6407 Bigelovii Wats.

6408 Californicum Nutt.

\section{ROSACEAE}

\section{Opulaster $\mathbf{3 0 4 9}$}

(Physocarpus)

6409 alternans (Jones) Heller

64 ro capitatus (Pursh) Kuntze

64I I monogynus (Torr.) Kuntze

(Neillia Torreyi Wats.)

6412 opulifolius (L.) Kuntze

64 I 3 panciflorus ('T. \& G.) Heller

(Neillia malvacea Greene)

64I4 pubescens Rydb. 

Spiraea 305\%

6415 betulifolia Pall.

64 I6 cinerascens Piper

6417 corymbosa Raf.

64 I 8 densiflora Nutt.

64 I9 Douglasii Hook.

6420 Nobleana Wats.

642 I Kamtschatica Pall.

6422 lucida Dougl.

[S. betulifolia of authors, not the Arctic species of Pall.

6423 Menziesii Hook.

6424 pyramidata Greene

6425 salicifolia L.

6426 latifolia Ait.

6427 tomentosa L.

6428 Virginiana Britton

\section{Lutkea $\mathbf{3 0 5 4}$}

(Eriogynia)

6429 Hendersoni (Canby) Greene 6430 pectinata (Hook.) Kuntze

\section{Petrophyton 3054a}

(Eriogynia in part)

643I caespitosum (Nutt.) Rydb.

6432 elatior (Wats.) Heller

Kelseya 3054b

6433 uniflora (Wats.) Rydb.

\section{Aruncus 3055}

6434 Aruncus (L.) Karst.

(Spiraea Aruncus L.)

Chamaebatiaria $\mathbf{3 0 5 6}$

6435 Millefolium Maxim.

Porteranthus 3058

(Gillenia)

6436 stipulatus (Muh1.) Britton

6437 trifoliatus (L.) Britton

Lyonothamnus $\mathbf{3 0 5 8 a}$

6438 asplenifolius Greene

6439 floribundus A. Gray

\section{Vaquelinia $306 \%$}

6440 Californica ('Torr.) Sargent (V. Torreyi Wats.)

\section{Holodiscus 3065}

(Spiraea in part)

644I ariaefolius (Smith) Greene

6442 australis Heller

6443 discolor (Pursh) Maxim.

6444 dumosus (Nutt.) Heller

\section{Cotoneaster $\mathbf{3 0 6 6}$}

6445 Pyracantha (L.) Spach.

\section{Crataegus (3066)}

6446 aestivalis (Walt.) T. \& G.

6447 apiifolia (Marsh.) Michx.

6448 austromontana Beadle

6449 berberifolia T. \& G.

6450 Biltmoreana Beadle

645I Boyntoni Beadle

6452 brachyacantha Sargent \&

6453 brevispina Dougl. Engelm.

$$
\text { (C. Douglasii Lind1.) }
$$

6454 Brownii Britton

6455 campestris Britton

6456 Chapmani (Beadle) Ashe

6457 coccinea L.

$645^{8}$ collina Chapm.

6459 Columbiana Howell

6460 cordata (Mill.) Ait.

646I Crus-galli L.

6462 angustifolia (Ehrh.) Sudw.

6463 fontanesiana (Spach) Wenz.

6464 linearis DC.

6465 ovalifolia Lindl.

6466

6467

6468

6469

6470 Eggertii Britton

647 r elliptica Ait.

6472 flabellata (Spach.) Rydb.

6473 flava Ait.

6474 integra Nash

6475 Harbinsoni Beadle

6476 macracantha (Lindl.) Lodd.

6477 Mohri Beadle

6478 mollis (T. \& G.) Scheele

6479 nitida Engelm.

6480 occidentalis Britton 

648I Oxyacantha L.

6482 Porteri Britton

6483 punctata Jacq.

6484 canescens Britton

6485 pyriformis Britton

6486 rivularis Nutt.

6487 rotundifolia (Ehrh.) Borck.

6488 saligna Greene

6489 Sargenti Beadle

6490 Sauratonae Ashe

649I silvicola Beadle

6492 spathulata Michx.

6493 tenuifolia Britton

6494 tomentosa L.

6495 pyrifolia A. Gray

6496 triflora Chapm.

6497 uniflora Muench.

(C. parvifolia Soland.)

6498 Vailiae Britton

6499 viridis L.

\section{Pyrus 30\%1}

6500 communis L.

\section{Malus (30\% 1)}

(Pyrus in part)

650I angustifolia (Ait.) Michx.

6502 coronaria (L.) Mill.

6503 Ioensis (Wood) Britton

6504 Malus (L.) Britton

6505 rivularis (Doug1.) Roem.

6506 Sonlardi (Bailey) Britton

\section{Sorbus (30\%1)}

(Pyrus in part)

6507 Americana Marsh.

6508 Aucuparia L.

6509 Californica Greene

65 Io dumosa Greene

65 I I occidentalis (Wats.) Greene

6512 sambucifolia (C. \& S.) Roem.

65 I 3 scopulina Greene

65 I4 subvestita Greene

\section{Aronia (30\%1)}

(Pyrus in part)

65 I5 arbutifolia (L.) Ell.

6516 nigra (Willd.) Britton

(Pyrus melanocarpa

\section{Heteromeles (30\%4)}

65 I 7 arbutifolia (Poir.) Roem.

\section{Amelanchier $\mathbf{3 0 7 6}$}

6518 alnifolia Nutt.

6519 Bakeri Greene

6520 Botryapium (L. f.) DC. (A. oblongifolia Roem.)

652 I Canadensis (L.) Medic.

6522 crenata Greene

6523 cuneata Piper

6524 Cusickii Fernald

6525 florida Lindl.

6526 glabra Greene

6527 Gormani Greene

6528 oligocarpa (Michx.) Roem.

6529 pallida Greene

6530 arguta Greene

653I polycarpa Greene

6532 prunifolia Greene

6533 pumila Nutt.

6534 rotundifolia (Michx.) Roem.

6535 rubescens Greene

6536 spicata (Lam.) Dec.

6537 Utahensis Koehne

6538 venulosa Greene

\section{Peraphyllum (30\%6)}

6539 ramosissimum Nutt.

\section{Neviusia 308\%}

6540 Alabamensis A. Gray

\section{Rubus 3083}

654I Alleghaniensis Porter

6542 Americanus (Pers.) Britton (R. triflorus Richards.)

6543 arcticus L.

6544 grandiflorus Ledeb.

6545 argutus Link

$$
\text { (R. frondosus Bigel.) }
$$

6546 Canadensis I .

6547 Chamaemorus L.

6548 cuneifolius Pursh

6549 deliciosus James

6550 Enslenii Tratt. (R. Baileyanus Britton)

6551 hesperius Piper

6552 hispidus Is. 

6553 invisus Bailey

6554 laciniatus Willd.

6555 lasiococcus A. Gray

6556 leucodermis Dougl.

6557 Millspaughii Britton

[According to Bailey, this is a synonym of $R$. Canadensis L. ]

$655^{8}$ nanus Wats.

6559 neglectus Peck

6560 Neo-Mexicanus A. Gray

6561 nigrobaccus Bailey

( $R$. villosus of authors, not Ait.)

6562 albinus Bailey

6563 nivalis Dougl.

6564 occidentalis $\mathrm{L}$.

6565 odoratus L.

6566 parviflorus Nutt.

(R. Nutkanus Moc.)

6567 velutinus (Brewer) Greene

6568 pedatus Smith

6569 sativus (Bailey) Brainerd

6570 saxatilis L.

657 I setosus Bigel.

6572 spectabilis Pursh

6573 Menziesii (Hook.) Wats.

6574 stellatus Smith

6575 strigosus Michx.

6576 caudatus Robinson \&

6577 trilobus Moc.

Schrenck 66I I ambigens Greene

6578 trivialis Michx.

6579 ursinus C. \& S.

6580 villosus Ait.

(R. Canadensis of authors, not L.) 658I Michiganensis Card $65^{82}$ roribaccus Bailey

$65^{8} 3$ vitifolius C. \& S.

\section{Dalibarda (308:3)}

6584 repens L.

(Rubus Dalibarda L.)

Fragaria $\mathbf{3 0 8 4}$

6585 Americana (Porter) Britton

6586 bracteata Heller

$65^{87}$ Californica C. \& S.

6588 Franciscana Rydb.

$65^{89}$ Canadensis Michx.
6590 Chiloensis (L.) Duchesne

6591 Scouleri (Wats.) Rydb.

6592 crinita Rydb.

6593 cuneifolia Nutt.

6594 firma Rydb.

6595 glauca (Wats.) Rydb.

6596 Helleri Holzinger

6597 pauciflora Rydb.

6598 platypetala Rydb.

6599 prolifica Baker \& Rydb.

6600 pumila Rydb.

660I sibbaldifolia Rydb.

6602 Terrae-novae Rydb.

6603 truncata Rydb.

6604 vesca $\mathrm{L}$.

6605 alba (Ehrh.) Rydb.

6606 Virginiana L.

6607 australis Rydb.

6608 Grayana (Vilmorin) Rydb.

(F. Virginiana var. Illinoensis

A. Gray)

\section{Duchesnea $\mathbf{3 0 8 5}$}

6609 Indica (Andr.) Focke (Fragaria Indica Andr.)

\section{Potentilla 3086}

66ro altaica Bunge

66I 2 argentea L.

6613 Arizonica Greene

66r4 atrorubens Rydb.

66I 5 atrovirens Rydb.

66I6 bicrenata Rydb.

66I 7 biennis Greene

66I 8 biflora Willd.

66rg bipinnatifida Dougl.

6620 platyloba Rydb.

662 I Blaschkeana Turcz.

6622 brevifolia Nutt.

6623 Breweri Wats.

6624 expansa Wats.

6625 Canadensis L.

6626 candida Rydb.

6627 Cascadensis Rydb.

6628 collina Weibl.

6629 Coloradensis Rydb.

6630 concinna Richards. 

663I divisa Rydb.

6632 concinnaeformis Rydb.

6633 crinita A. Gray

6634 ctenophora Rydb.

6635 decurrens (Wats.) Rydb.

6636 dissecta Pursh

6637 glaucophylla(Lehm.)Wats. $663^{8}$ Drummondii Lehm.

6639 effusa Dougl.

6640 emarginata Pursh

664 I etomentosa Rydb.

6642 fastigiata Nutt.

6643 filicaulis (Nutt.) Rydb.

6644 flabellifolia Hook.

6645 flabelliformis Lehm.

6646 fragiformis Willd

6647 glabrella Rydb.

(P. Pennsylvanica var. glabrata Wats.)

6648 glomerata Aven Nelson

6649 gracilipes Piper.

6650 gracilis Doug1.

6651 Grayi Wats.

6652 Hippiana Lehm.

6653 propinqua Rydb. (P. diffusa A. Gray)

6654 Hookeriana Lehm.

6655 inclinata Vill.

6656 intermedia L.

6657 jucunda Aven Nelson

6658 lactea Greene

6659 Lemmoni (Wats.) Greene

6660 leucocarpa Rydb. (P. millegrana Engelm.)

666I litoralis Rydb.

6662 longipedunculata Rydb.

6663 Macounii Rydb.

6664 millefolia Rydb.

6665 minutifolia Rydb.

6666 monidensis Aven Nelson

6667 Monspeliensis L.

6668 multifida L.

6669 multijuga Lehm.

6670 multisecta (Wats.) Rydb.

667 I nana Willd.

6672 nemoralis Nestl.

6673 Newberryi A. Gray

6674 arenicola Rydb.
6675 Nicoletti (Wats.) Sheldon

6676 nivea $\mathrm{L}$.

6677 macrophylla Ser.

6678 Norvegica L.

6679 Nuttallii Lehm.

6680 glabrata Lehm.

668I paradoxa Nutt.

6682 pectinisecta Rydb.

6683 Pennsylvanica L.

6684 arachnoidea Lehm.

6685 strigosa Pursh

6686 pentandra Engelm.

6687 pinnatisecta (Wats.)

6688 Plattensis Nutt.

Aven Nelson

6689 procumbens Sibth.

6690 pseudosericea Rydb.

669 I pulchella $\mathrm{R}$. Br.

6692 pulcherrima Lehm.

6693 pumila Poir.

6694 quinquefolia Rydb.

6695 ramulosa Rydb.

6696 Ranunculus Lange

6697 recta L.

6698 reptans $\mathrm{L}$.

6699 rivalis Nutt.

6700 Robbinsiana Oakes

6701 rosulata $\mathrm{Rydb}$.

6702 rubens (Crantz) Vill.

(P. maculata Pourr)

6703 rubricaulis Lehm.

6704 rupincola Osterhont

6705 saximontana Rydb.

6706 saxosa Greene

6707 Sierrae-Blancae Wooton \&

6708 simplex Nestl.

Rydb.

6709 Sommerfeltii Lehm.

67I subjuga Rydb.

67I I subviscosa Greene

67 I 2 sulphurea Lam.

67I3 tenerrima Rydb.

6714 Thurberi A. Gray

6715 uniflora Ledeb.

6716 Vahliana Lehm.

6717 villosa Pall.

6718 chrysocoma Rydb.

6719 virgultula Aven Nelson 

6720 viridescens $\mathrm{Rydb}$.

672 I Wheeleri Wats.

6722 viscidula $R y d b$.

6723 Wyomingensis Aven Nelson

\section{Argentina (3086)}

(Potentilla in part)

6724 Anserina (L.) Rydb.

6725 concolor (Ser.) Rydb.

6726 grandis (T. \& G.) Rydb.

6727 Egedii (Wormsk.) Rydb.

\section{Comarum (3086)}

(Potentilla in part)

6728 palustre L.

6729 villosum Pers

\section{Dasiophora (3086)}

(Potentilla in part)

6730 fruticosa (L.) Rydb.

673I monticola Rydb.

6732 tenuifolia (Willd.) Rydb.

\section{Drymocallis (3086)}

(Potentilla in part)

6733 arguta (Pursh) Rydb.

6734 Ashlandica (Greene) Rydb.

6735 convallaria Rydb.

6736 cuneifolia Rydb.

6737 fissa (Nutt.) Rydb.

$673^{8}$ glabrata Rydb.

6739 glandulosa (Lindl.) Rydb.

6740 incisa (Lindl.) Rydb.

674I monticola Rydb.

6742 glutinosa (Nutt.) Rydb.

6743 Neo-Mexicana Rydb.

6744 Hanseni (Greene) Rydb.

6745 pseudorupestris Rydb.

6746 intermedia Rydb.

6747 reflexa (Greene) Rydb.

6748 rhomboidea Rydb.

6749 Wrangelliana (F. \& A.) Rydb.

\section{Horkelia 308\%}

(Including Ivesia)

6750 argyrocoma Rydb.

675 I Baileyi (Wats.) Rydb.

6752 setosa (Wats.) Rydb.

6753 Bolanderi A. Gray
6754 Californica C. \& S.

6755 campestris (Jones) Rydb.

(Potentilla Utahensis var. campestris Jones)

6756 capitata Lindl.

6757 caruifolia Rydb.

$675^{8}$ chaetophora Rydb.

6759 Congdonis Rydb.

6760 congesta Hook.

6761 cuneata Lindl.

6762 daucifolia (Greene) Rydb.

6763 elata (Greene) Rydb.

6764 eremica (Coville) Rydb.

6765 flavescens Rydb.

6766 frondosa (Greene) Rydb.

6767 fusca Lindl.

6768 tenella Wats.

6769 Gordonii Hook.

6770 alpicola Rydb.

6771 megalopetala Rydb.

6772 Hendersoni Howell

6773 hirsuta Lindl.

6774 Howellii (Greene) Rydb.

6775 Kingii (Wats.) Rydb.

6776 laxiflora (Drew) Rydb.

6777 lycopodioides (A. Gray) Rydb.

6778 Michneri (Greene) Rydb.

6779 Muirii (A. Gray) Rydb.

6780 mutabilis 'T. S. Brandegee

678 I Parryi (Wats.) Rydb.

6782 parviflora Nutt.

6783 Pickeringii (A. Gray) Rydb.

6784 pinetorum (Coville) Rydb.

6785 platycalyx Rydb.

6786 platypetala Rydb.

6787 pseudocapitata Rydb.

6788 puberula (Greene) Rydb.

6789 purpurascens Wats.

6790 pygmaea (A. Gray) Rydb.

679I scandularis Rydb.

6792 sericata Wats.

6793 sericea (A. Gray) Rydb.

6794 sericoleuca Rydb.

6795 Shockleyi (Wats.) Rydb.

6796 tenella (Wats.) Rydb.

6797 tenuiloba (Torr.) A. Gray

6798 tridentata Torr.

6799 unguiculata (A. Gray) Rydb. 

6800 Utahensis (Wats.) Rydb.

68or Webberi (A. Gray) Rydb.

\section{Stellariopsis (308\%)}

6802 santalinoides (A. Gray) Rydb. (Ivesia santalinoides A. Gray)

\section{Comarella (3087)}

6803 multifoliolata (Torr.) Rydb. (Ivesia depauperata A. Gray) 6804 sabulosa (Jones) Rydb.

(Iresia sabulosa Jones)

\section{Sibbaldia $\mathbf{3 0 8 9}$}

6805 procumbens L.

\section{Sibbaldiopsis 3089a}

6806 tridentata (Soland.) Rydb.

(Potentilla tridentata Soland.)

\section{Chamaerhodos $\mathbf{3 0 9 1}$}

6807 erecta (L.) Bunge

6808 Nuttallii T. \& G.

\section{Purpusia 3091a}

6809 saxosa T. S. Brandegee

\section{Waldsteinia $309 \%$}

681o fragarioides (Michx.) Tratt.

68I I lobata T. \& G.

6812 parviflora Small

\section{Geum 3094}

68 I 3 anemonoides Willd.

68I4 calthifolium Menzies

68I5 congestum T. \& G.

68I6 rotundifolium. T. \& G.

6817 Canadense Jacq.

$$
\text { (G. album Gmel.) }
$$

6818 flavum (Porter) Bicknell

68I9 geniculatum Michx.

6820 macrophyllum Willd.

682 I Oregonense (Scheutz) Rydb.

6822 radiatum Michx.

6823 Peckii (Pursh) A. Gray

6824 rivale $\mathrm{L}$.

6825 scopulorum Greene

6826 strictum Ait.

6827 urbanum L.

6828 vernum (Raf.) T. \& G.
Sieversia (3094)

(Geum in part)

6829 ciliata (Pursh) G. Don (Geum triflorum Pursh)

6830 glacialis R. Br.

$683 \mathrm{I}$ humilis $\mathrm{R}$. Br.

6832 Rossii R. Br.

6833 rotundifolia (Langsd.) C. \& S.

6834 sericea Greene

6835 turbinata (Rydb.) Greene

Fallugia 3095

6836 paradoxa (Don) Endl.

6837 acuminata Wooton

\section{Cowania $\mathbf{3 0 9 6}$}

6838 ericaefolia Torr.

6839 Mexicana D, Don

6840 plicata D. Don

\section{Dryas 309 \%}

684I Drummondii Richards.

6842 integrifolia Vahl.

6843 octopetala L.

6844 integrifolia C. \& S.

\section{Cercocarpus 3098}

6845 betuloides Nutt.

6846 breviflorus A. Gray

6847 intricatus Wats.

6848 ledifolius Nutt.

6849 parvifolius Nutt.

6850 paucidentatus (Wats.) Britton

685I Traskiae Eastwood

\section{Adenostoma $\mathbf{3 0 9 9}$}

6852 fasciculatum H. \& A.

6853 obtusifolium Wats.

6854 sparsifolium Torr.

\section{Coleogyne $\mathbf{3 1 0 0}$}

6855 ramosissima Torr.

\section{Kunzia 310\%}

$$
\text { (Purshia) }
$$

6856 glandulosa (Curran) Greene

6857 tridentata (Pursh) Spreng

\section{Chamabatia $310: 3$}

6858 foliolosa Benth.

6859 australis T. S. Brandegee 

Ulmaria $\mathbf{3 1 0 4}$

(Spiraea in part)

6860 rubra Hill

(S. lobata Gronov.)

686I Ulmaria (L.) Barnhart

Filipendula (3104)

6862 lobata Maxim.

6863 occidentalis Wats.

\section{Alchemilla 3105}

6864 alpina L.

6865 arvensis (L.) Scop.

\section{Agrimonia $\mathbf{3 1 0 6}$}

6866 Brittoniana Bicknell

6867 hirsuta (Muhl.) Bicknell

6868 incisa T. \& G.

6869 mollis ('T. \& G.) Britton

6870 Bicknellii Kearney

687 I parviflora Soland.

6872 pumila Muhl.

6873 striata Michx.

[Agrimonia Eupatoria does not occur in this country.]

\section{Sanguisorba $\mathbf{3 1 1 1}$}

\section{(Poterium)}

6874 annua (Nutt.) Holzinger

6875 Canadensis L.

6876 latifolia Coville

6877 media L.

6878 Sanguisorba (L.) Britton

6879 Sitchensis C. A. Meyer.

\section{Acaena 3116}

688 o trifida R. \& P.

\section{Rosa 3119}

688I acicularis Lindl.

$$
\text { (R. Engelmanni Wats.) }
$$

6882 Arkansana Porter

6883 blanda Ait.

6884 bracteata Wendl.

6885 Californica C. \& S.

6886 glabrata S. B. Parish

6887 canina L.

6888 Carolina L.

6889 cinnamomea L.
6890 Fendleri Crepin

689 I foliolosa Nutt.

6892 gratissima Greene

6893 gymnocarpa Nutt.

6894 pubescens Wats.

6895 humilis Marsh.

6896 villosa Best

6897 laevigata Michx.

6898 lucida Ehrh.

6899 MacDougali Holzinger

- 6900 Macounii Greene

69o I manca Greene

6902 melina Greene

6903 multiflora Thunb.

6904 nitida Willd.

6905 Nutkana Presl

6906 pisocarpa A. Gray

- 6907 pratincola Greene

6908 rubiginosa L.

6909 Sayi Schwein.

6910 Sonomensis Greene

69I I setigera Michx.

6912 tomentosa T. \& G.

6913 stellata Wooton

6914 suffulta Greene

69r5 Woodsii Lindl.

\section{Osmaronia $\quad 31 \% \%$}

(Nuttallia)

6916 cerasiformis ('T. \& G.) Greene

Prunus 31\%5

6917 Alabamensis Mohr

69r8 Allegheniensis Porter

6919 Americana Marsh.

6920 lanata Sudw.

$692 \mathrm{I}$ angustifolia Marsh. (P. Chicasa Michx.)

6922 Armeniaca L.

6923 Avium L.

6924 Besseyi Bailey

6925 Capollin DC.

6926 Caroliniana (Mill.) Ait.

6927 Cerasus L.

6928 corymbulosa Rydb.

6929 cuneata Raf.

6930 demissa (Nutt.) Walp.

6931 domestica I. 

6932 emarginata (Dougl.) Walp.

6933 villosa Sudw.

( $P$. emarginata var. mollis Brewer)

6934 fasciculata A. Gray

6935 Fremontii Wats.

6936 glandulosa T. \& G.

6937 gracilis Engelm. \& Gray

6938 Gravesii Small

6939 hortulana Bailey

6940 Mineri Bailey

694I ilicifolia (Nutt.) Walp.

6942 integrifolia Sudw.

6943 injucunda Small

6944 insititia L.

6945 Mahaleb L.

6046 maritima Wang.

6947 minutiflora Engelm.

6948 nigra Ait.

6949 Oregana Greene

6950 Pennsylvanica L. f.

$695^{1}$ pumila L.

6952 rivularis Scheele

6953 salicifolia H. B. K.

(P. Capuli Cav.)

6954 serotina Ehrh.

6955 neomontana Sudw. (P. serotina Smallii Britton)

6956 sphaerocarpa Sw.

6957 spinosa L.

6958 subcordata Benth.

6959 Kelloggii Lemmon

6960 umbellata Ell.

696I Virginiana L.

6962 Watsoni Sargent

\section{Amygdalus (31\%5)}

6963 Andersoni (A. Gray) Greene 6964 Persica L.

\section{Chrysobalanus $31 \%$ g}

6965 Icaco L.

6966 oblongifolius Michx.

\section{MIMOSACEAE}

\section{Zygia 3159}

(Pithecolobium)

6967 brevifolia (Benth.) Sudw.

6968 flexicaulis (Benth.) Sudw.

(Acacia flexicaulis Benth.)
6969 Guadalupensis (Desv.) Heller 6970 Unguis-Cati (L.) Sudw.

\section{Albizzia $\mathbf{3 1 6 0}$}

697x Julabrissin Durazz.

\section{Calliandra 3161}

6972 conferta Benth.

6973 eriophylla Benth.

6974 humilis Benth.

6975 reticulata A. Gray

6976 Schottii Torr.

\section{Lysiloma 316\%}

6977 latisiliqua (L.) Benth.

\section{Acacia $\mathbf{3 1 6 3}$}

6978 amentacea DC.

6979 Berlandieri Benth.

6980 constricta Benth.

6981 Emoryana Benth.

6982 Farnesiana (L.) Willd.

6983 filiculoides (Cav.) Trelease

(A. filicina Willd.)

6984 Greggii A. Gray

6985 malacophylla Benth.

6986 millefolia Wats.

6987 Schottii Torr.

6988 tortuosa Willd.

6989 Wrightii Benth.

\section{Leucaena $\mathbf{3 1 6 4}$}

6990 glauca (L.) Benth.

699 I pulverulenta(Schlecht.)Benth. 6992 retusa Benth.

\section{Morongia $\mathbf{3 1 6 5}$}

(Schrankia)

6993 aculeata (Willd.) Heller 6994 angustata (T. \& G.) Britton 6995 Floridana (Chapm.) Heller 6996 horridula (Michx.) Heller 6997 angularis (Chapm.) Heller 6998 Roemeriana (Scheele) Heller

(Schrankia platycarpa A. Gray) 6999 uncinata (Willd.) Britton

\section{Mimosa 3166}

7000 acanthocarpa Benth. 7001 Berlandieri A. Gray 

7002 biuncifera Benth.

7003 borealis A. Gray 7004 Texana A. Gray 7005 depauperata Benth. 7006 dysocarpa Benth. 7007 Emoryana Benth. 7008 flexuosa Benth. 7009 fragrans A. Gray 7oro Grahami A. Gray 70I Lemmoni A. Gray 7012 Lindheimeri A. Gray 7013 malacophylla A. Gray 7014 glabrata Benth. 7015 monancistra Benth.

7016 Pringlei Wats.

7017 pudica L.

7018 strigillosa T. \& G.

7019 Wrightii A. Gray 7020 zygophylla Benth.

\section{Acuan 316\%}

(Desmanthus)

7021 acuminata (Benth.) Kuntze 7022 depressa (H. \& B.) Kuntze 7023 Fendleri (Wats.) Heller 7024 glandulosa (Michx.) Heller 7025 Illinoensis (Michx.) Kuntze (D. brachylobus Benth.)

7026 Jamesii (T. \& G.) Kuntze 7027 leptoloba (T. \& G.) Kuntze 7028 obtusa (Wats.) Heller 7029 reticulata (Benth.) Kuntze 7030 velutina (Scheele) Kuntze. 703I virgata Med.

\section{Neptunia 3168}

7032 Floridana Small

7033 Lindheimeri Robinson

7034 lutea (Leavenw.) Benth.

7035 oleracea Lour.

7036 pubescens Benth.

7037 tenuis Benth.

\section{Prosopis 3169}

7038 cinerascens Benth.

7039 glandulosa Torr.

7040 juliflora (Sw.) DC. 704 I odorata Torr. \& Frem.

(P. pubescens Benth.)
7042 velutina Wooton

CAESALPINACEAE

Cercis 3\%23

7043 Canadensis L. *

7044 pubescens Pursh

7045 occidentalis Torr.

7046 reniformis Engelm.

\section{Cassia 3233}

7047 armata Wats.

7048 Bahamensis Mill.

7049 bauhinioides A. Gray

7050 biflora L.

$705 \mathrm{I}$ confinis Greene

7052 Covesii A. Gray

7053 leptocarpa Benth.

7054 ligustrina Benth.

7055 Lindheimeriana Scheele

7056 Marylandica L.

7057 occidentalis L.

7058 pumilio A. Gray

7059 Roemeriana Scheele

7060 Tora L.

706r Wislizeni A. Gray

\section{Chamaecrista}

(3233)

(Cassia in part)

7062 aspera (Muhl.) Greene

7063 Mohrii Pollard

7064 chamaecristoides (Mill.)Greene

(Cassia depressa Pollard

7065 cinerea (C. \& S.) Pollard

7066 fascicularis (Michx.) Greene

7067 grammica (Spreng.) Pollard

7068 Greggii (A. Gray) Pollard

7069 Mississippiensis Pollard

7070 multipinnata (Pollard) Greene

707 I Nashii (Pollard) Greene

7072 nictitans (L.) Moench.

7073 procumbens ( $\mathrm{L}_{40}$ ) Greene

7074 robusta Pollard

7075 Simpsoni Pollard

7076 Wrightii (A. Gray) Pollard

\section{Krameria $\mathbf{3 2 3 9}$}

7077 canescens A. Gray

7078 parvifolia Benth. 

7079 ramosissima Wats.

7080 recundiflora DC.

Gleditsia $\mathbf{3 \% 4 0}$

708I aquatica Marsh.

7082 triacanthos L.

7083 brachycarpos Michx.

7084 laevis (Loud.) Sudw.

Gymmocladus $\mathbf{3 2 4 1}$

7085 dioicus (L.) Koch

(G. Canadensis Lam.)

Parkinsonia $\mathbf{3 2 4 6}$

7086 aculeata L.

7087 microphylla Torr.

\section{Cercidium $\quad \mathbf{3 \% 4 9}$}

(Parkinsonia in part)

7088 floridum Benth.

7089 Texanum A. Gray

7090 Torreyanum (Wats.) Sargent

\section{Hofmanseggia 325\%}

709I brachycarpa A. Gray

7092 caudata A. Gray

7093 drepanocarpa A. Gray

7094 Drummondii T. \& G.

7095 Falcaria Cav.

7096 capitata Fisher

7097 Pringlei Fisher

7098 Rusbyi Fisher

7099 gladiata Benth.

7roo Jamesii 'T. \& G.

7 IOI Popinoensis Fisher

7102 melanosticta A. Gray

$7{ }_{103}$ Parryi Fisher

7 I04 microphylla Torr.

7 I05 oxycarpa Benth.

7106 platycarpa Benth.

7107 stricta Benth.

7108 demissa A. Gray .

7109 Texensis Fisher

Caesalpinia $\mathbf{3 2 5 4}$

7 I Io pauciflora B. \& H.

7IrI pulcherrima Sw.

\section{Guilandina (3\%54)}

7 II 2 Bonduc L.

(Caesalpinia Bonduc Roxb.) 7r48 leucantha T. \& G.

\section{PAPILIONACEAE}

Sophora 3\%94

7 II 3 affinis T. \& G.

7 II 4 Arizonica Wats.

7 II 5 secundiflora (Cav.) DC.

7 II6 sericea Nutt.

7 I 7 stenophylla A. Gray

7 II 8 tomentosa L.

Cladrastis 3298

7 II9 litea (Michx. f.) Koch (C. tinctoria Raf.)

\section{Thermopsis $\mathbf{3 3 0 9}$}

7I 20 annulocarpa Aven Nelson

7 I 2 I arenosa Aven Nelson

71 22 argentata Greene

7 I 23 Californica Wats.

7 I 24 Caroliniana Curtis

7 1 25 divaricarpa Aven Nelson

7I26 fraxinifolia M. A. Curtis

7127 gracilis Howell

7128 macrophylla H. \& A.

7 I 29 mollis (Michx.) M. A. Curtis

7r 30 montana Nutt.

7I 3 I pinetorum Greene

7132 rhombifolia (Nitt.) Richards.

7I33 robusta Howell

7I34 velutina Greene

\section{Baptisia 3310}

7I 35 alba (L.) R. Br.

7 I 36 australis (L.) R. Br.

7137 minor T. \& G.

71 38 bracteata Ell.

(B. leucophaea Nutt.)

7 I 39 calycosa Canby

7140 villosa Canby

7I4I confusa Pollard \& Ball

(B. lanceolata var. Texana

Holzinger)

7 I42 cuneata Small

7 I 43 Hugeri Small

7 I 44 laevicaulis (A. Gray) Small

7I45 lanceolata Ell.

7I46 uniflora 'T. \& G.

7147 Lecontei T. \& G. 

7r49 megacarpa Chapm. 7 I 50 microphylla Nutt. $715 \mathrm{I}$ axillaris Canby 7 I 52 perfoliata $\mathrm{R}$. $\mathrm{Br}$. 7 I53 lobata Canby 7 I 54 Serenae M. A. Curtis 7 I55 simplicifolia Croom 7 I 56 sphaerocarpa Nutt. 7 I57 sulphurea Engelm. 7 I $5^{8}$ tinctoria (L.) R. Br. 7 r 59 villosa (Walt.) Nutt.

\section{Xylothermia $\mathbf{3 3 1 1}$}

(Pickeringia)

7 I60 montana (Nutt.) Greene

\section{Crotalaria $\mathbf{3 3 6 1}$}

7I6I incana L.

7 I62 lupulina Raf.

7163 maritima Chapm.

7164 Pringlei A. Gray

7165 pumila Ortega

7 I66 Purshii DC.

7167 retusa L.

7 I68 rotundifolia (Walt.) Poir. (C. avalis Pursh)

7r69 sagittalis L.

\section{Lupinus 3363}

7r 70 adsurgens Drew

7I 7 I aduncus Greene

7172 affinis Agardh

7173 albicanlis Dougl.

7I74 albifrons Benth.

7175 collinus Greene

7176 alpestris Aven Nelson

7177 alsophilus Greene

7178 ammophilus Greene

7I79 Andersoni Wats.

7180 arboreus Sims

$718 \mathrm{I}$ arcticus Wats.

7182 argenteus Pursh

7183 aridus Dougl.

7 r84 Lobbii Wats.

7 185 Arizonicus Wats.

7r86 Bakeri Greene

7187 barbiger Wats.

7188 bicolor Lindl.

7189 brevicaulis Wats. 7rgo Breweri A. Gray

7 I9I Burkei Wats.

7 I92 caespitosus Nutt.

7193 calcaratus Kellogg

7 r94 canescens Howell

7195 capitatus Greene

7196 carnosulus Greene

7197 Chamissonis Esch

7198 longebracteatus Wats.

7199 longifolius Wats.

7200 citrinus Kellogg

7201 concinnus Agardh

$72 \mathrm{O} 2$ confertus Kellogg

7203 Wrightii Greene

7204 Covillei Greene

7205 Cusickii Wats.

7206 decumbens Torr.

7207 densiflorus Benth.

7208 diffusus Nutt.

7209 Douglasii Agardh

72 Io Drakei Howell

72 I I Elmeri Greene

7212 eminens Greene

7213 erectus Henderson

7214 eximius Davy

72 I 5 fallax Greene

7216 flexuosus Lindl.

7217 floribundus Greene

72 I 8 formosus Greene

7219 Bridgesii (Wats.) (ireene

7220 Franciscanus Greene

722 I fulcratus Greene

7222 gracilentus Greene

7223 gracilis Agardh

7224 gratus Greene

7225 Grayi Wats.

7226 Havardi Wats.

7227 Hellerae Heller

7228 Helleri Greene

7229 hirsutissimus Benth.

7230 holosericeus Nutt.

723I humicola Aven Nelson

7232 ingratus Greene

7233 jucundus Greene

7234 Kingii Wats.

7235 latifolius Agardh

7236 laxiflorus Dongl.

7237 montanus Howell 

7238 lepidus Dougl.

7239 leptophyllus Benth.

7240 leucophyllus Dougl.

724 I ligulatus Greene

7242 barbatus Henderson

7243 littoralis Dougl.

7244 Lobbii A. Gray

7245 longipes Greene

7246 Ludovicianus Greene

7247 luteolus Kellogg

7248 Lyallii A. Gray

7249 Dananus Wats.

7250 magnificus Jones

$725 \mathrm{I}$ glarecola Jones

7252 magnus Greene

7253 malacophyllus Greene

7254 meionanthus A. Gray

7255 Micensis Jones

7256 Michneri Greene

7257 micranthus Dougl.

$725^{8}$ microphyllus Wats.

7259 microcarpus Sims

7260 minimus Dougl.

726I monticola Rydb.

7262 mucronatulus Howell

7263 myrianthus Greene

7264 nanus Dougl.

7265 nemoralis Greene

7266 Neo-Mexicanus Greene

7267 Nootkatensis Donn

7268 Unalaskensis Wats.

7269 Nuttallii Wats.

7270 onustus Wats.

727 I oreophilus Greene

7272 ornatus Dongl.

7273 pachylobus Greene

7274 Palmeri Wats.

7275 parviflorus Nutt.

7276 perennis L.

7277 occidentalis Wats.

7278 pinetorum Jones

7279 pseudoparviflorus Rydb.

7280 Plattensis Wats.

728I polycarpus Greene

7282 polyphyllus Lindl.

7283 Pondii Greene

7284 propinquus Greene

7285 psoraloides Pollard
7286 pusillus Pursh

7287 retrorsus Henderson

7288 rivularis Dougl.

7289 Sabinii Dougl.

7290 saxosus Howell

729 I sericeus Pursh

7292 Shockleyi Wats.

7293 Sitgreavesii Wats.

7294 sparsiflorus Benth.

7295 Stiversii Kellogg

7296 subcarnosus Hook.

7297 sulphureus Dougl.

7298 Tidestromii Greene

7299 trifidus Torr.

7300 truncatus Nutt.

730 I umbellatus Greene

7302 uncialis Wats.

7303 variicolor Steud.

7304 villosus Willd.

7305 volcanicus Greene

7306 Wyethii Wats.

Genista 3366

7307 tinctoria L.

\section{Ulex $337 \%$}

7308 Europaeus L.

Cytisus $\quad 33 \% 3$

7309 scoparius (L.) Link.

Medicago $\mathbf{3 3}$ \%

73ro apiculata Willd.

73II Arabica All.

$73^{12}$ deltoidea Michx.

73 I 3 denticulata Willd.

73I 4 intertexta Mill.

73I 5 lupulina L.

7316 nigra Willd.

73I 7 sativa $\mathrm{L}$.

\section{Melilotus 3380}

7318 alba Desv.

7319 Indica (L.) All.

7320 officinalis (L.) Lam.

\section{Trifolium 3381}

732 I aciculare Nutt.

7322 albopurpureum T. \& G.

7323 altissimum Dougl. 

7324 amoenum Greene

7325 amphianthum T. \& G.

7326 amplectens T. \& G.

7327 Andersonii A. Gray

7328 andinum Nutt.

7329 anemophilum Greene

7330 appendiculatum Loja

733 I Arizonicum Greene

7332 arvense $\mathrm{L}_{4}$.

7333 attenuatum Greene

7334 barbigerum Torr.

7335 Andrewsii A. Gray

7336 Beckwithii Brewer

7337 Bejariense Moricand

7338 bifidum A. Gray

7339 decipiens Greene

7340 Bolanderi A. Gray

734I Brandegei Wats

7342 Breweri Wats.

7343 calophyllum Greene

7344 Carolinianum Michx.

7345 Catalinae Wats.

7346 caurinum Piper

7347 ciliatum Nutt.

7348 ciliolatum Benth.

7349 Columbianum Greene

7350 cyathiferum Lindl.

735 I dasyphyllum T. \& G.

7352 depauperatum Desv.

7353 amplectens Wats.

7354 dianthum Greene

7355 dichotomum H. \& A.

7356 diversifolium Nutt.

7357 Elmeri Greene

$735^{8}$ eriocephalum Nutt.

7359 exile Greene

7360 Fendleri Greene

736 r fimbriatum Lindl.

7362 flavulum Greene

7363 Franciscannm Greene

7364 furcatum Lindl.

7365 Gambelii Nutt.

7366 geminiflorum Greene

7367 gracilentum T. \& G.

7368 iuconspicuum Fernald

7369 gymnocarpon Nutt.

7370 Hallii Howell

737I Hanseni Greene
7372 Harneyensis Howell

7373 Haydeni Portes

7374 heterodon T. \& G.

7375 Howellii Wats.

7376 hybridum L.

7377 hydrophilum Greene

7378 incarnatum L.

7379 Kingii Wats.

7380 lacerum Wats.

7381 laciniatum Greene

7382 angustatum Greene

$73^{8} 3$ latifolium (Hook.) Greene

7384 Lemmoni Wats.

7385. lilacinum Greene

7386 longipes Nutt.

7387 brachypus Wats.

7388 macilentum Greene

7389 Macraei H. \& A.

7390 dichotomum Brewer

739 I macrocephalum (Pursh) Poir.

7392 majus (Howell) Greene

7393 medium L.

7394 megacephalum Nutt.

7395 melantherum H. \& A.

7396 microcephalum Pursh

7397 microdon H. \& A.

7398 pilosum Eastwood

7399 minutiflorum Greene

7400 monanthum A. Gray

740 Monoense Greene

$74 \mathrm{O} 2$ Montanense Rydb.

7403 Morleyanum Greene

7404 nanum Torr.

7405 nemorale Greene

7406 obtusiflorum Hook.

7407 oliganthum Stend.

7408 Sonomense Greene

7409 olivaceum Greene

7410 Oreganum Howell

74I I Palmeri Wats.

741 2 Parryi A. Gray

74I3 pauciflorum Nutt.

74I4 phaeocephalum Greene

74I5 pinetorum Greene

74I6 Plummerae Wats.

74I7 plumosum Dougl.

7418 polyodon Greene

74I9 pratense L. 

7420 productum Greene

742 I pusillum Greene

7422 reflexum L.

7423 repens L.

7424 roscidum Greene

7425 rostratum Greene

7426 Rusbyi Greene

7427 atrorubens Greene

7428 Rydbergii Greene

7429 saxicolum Small

7430 scabrellum Greene

743I segetum Greene

7432 spinulosum Dougl

7433 stenophyllum Nutt.

7434 stoloniferum Muhl.

7435 subsalinum Greene

7436 tridentatum Lindl.

7437 triflorum Greene

7438 trilobatum Jepson

7439 trimorphum Greene

7440 triste Nutt.

744I truncatum Greene

7442 ultramontanum Greene

7443 variegatum Nutt.

7444 major Loja

7445 Virginicum Small

7446 virescens Greene

7447 Watsoni Loja

7448 Wormskjoldii Lehm.

\section{Chrysaspis (3381)}

(Trifolium in part)

7449 agraria (L.) Greene

7450 aurea (Poll.) Greene

745I dubia (Sibth.) Greene

(T. procumbens var. minus A. Gray) $745^{2}$ procumbens (L.) Desv.

\section{Lotus 3389}

(Hosackia)

7453 Americanus (Nutt.) Bisch.

(H. Purshiana Benth.)

7454 Argensis Coville

7455 argophyllus (A. Gray) Greene

7456 argyraeus Greene

7457 balsamiferus (Kellogg) Greene $745^{8}$ Benthami Greene

(H. cytisoides Benth.)
7459 Bioletti Greene

7460 Cedrosensis Greene

746I confinis Greene

7462 corniculatus L.

7463 crassifolius (Benth.) Greene

7464 Davidsoni Greene

7465 dendroideus Greene

7466 denticulatus (Drew) Greene

7467 diffusus (A. Gray) Heller

7468 distichus Greene

7469 Douglasii Greene

(H. decumbens Benth.)

7470 eriophorus Greene

747 I formosissimus Greene (H. gracilis Benth.)

7472 Fremonti A. Gray) Heller

7473 glaber ('Torr.) Greene

7474 grandiflorus (Benth.) Greene

7475 Guadalupensis Greene

7476 hamatus Greene

7477 Haydeni (Orcutt) Greene

7478 Heermani (Dur. \& Hilg.)

7479 Helleri Britton

7480 hirtellus Greene

748 I humistratus Greene (H. brachycarpa Benth.)

7482 incanus (Torr.) Greene

7483 junceus Benth Greene

7484 lathyroides (Dur. \& Hilg.)

Greene

7485 leucophaeus Greene

7486 leucophyllus Greene (H. sericea Benth.)

7487 macranthus Greene

7488 Mearnsii Britton

7489 micranthus Benth.

(H. parviflora Benth.)

7490 mollis Greene

749I nanus (Wats.) Heller

7492 Neo-Mexicanus Greene

7493 Nevadensis (Wats.) Greene

7494 niveus Greene

7495 nudatus (Vasey \& Rose) Greene

7496 nudiflorus (Nutt.) Greene

7497 Nuttalianus Greene

(H. prostrata Nutt.)

7498 oblongifolius (Benth.) Greene 

7499 occultus Greene

7500 ornithopus Greene

750 I pinnatus Hook.

7502 procumbcns Greene

7503 puberulus (Benth.) Greene

7504 rigidus (Benth.) Greene

7505 rubellus (Nutt.) Greene

7506 salsuginosus Greene

(H. maritima Nutt.)

7507 stipularis (Benth.) Greene

7508 strigosus (Nutt.) Greene

7509 tomentosus (H. \& A.) Greene

75 Io Torreyi (A. Gray) Greene

75II trispermus Greene

$75^{12}$ Veatchii Greene

7513 venustus (Eastwood) Heller

75I4 Wrangellianus F. \& M. (H. subpinnata T. \& G.)

7515 Wrightii (A. Gray) Greene

\section{Indigofera $\mathbf{3 3 9 1}$}

\section{I6 Anil L.}

75I7 leptosepala Nutt.

$75^{18}$ Lindheimeriana Scheele

7519 sphaerocarpa A. Gray

7520 subulata Vahl.

752 I tinctoria L.

\section{Psoralea $\mathbf{3 3 9 \%}$}

7522 argophylla Pursh

7523 decumbens A. Gray.

7524 Californica Wats.

$75^{2} 5$ campestris Nutt.

7526 canescens Michx.

7527 castorea Wats.

7528 collina Rydb.

7529 cuspidata Pursh

7530 cyphocalyx A. Gray

7531 digitata Nutt.

7532 Douglasii Greene

7533 esculenta Pursh

7534 floribunda Nutt.

7535 hypogaea Nutt.

7536 scaposa A. Gray

7537 lanceolata Pursh

7538 linearifolia T. \& G.

7539 robusta Coult.

7540 lupinellus Michx.

754I macrostachya DC.
7542 mephitica Wats.

7543 micrantha A. Gray

7544 monticola Greene

7545 multijuga Ell.

7546 obtusiloba T. \& G.

7247 Onobrychis Nutt.

7548 orbicularis Lindl.

7549 pedunculata (Mill.) Vail ( $P$. melilotoides Michx.)

$755^{\circ}$ physodes Dougl.

755 I Purshii Vail

7552 Reverchoni Wats.

7553 rigida Parish

7554 rhombifolia T. \& G.

7555 simplex Nutt.

7556 stipulata T. \& G.

7557 strobilina H. \& A.

$755^{8}$ subacaulis T. \& G.

7559 tenuiflora Pursh

7560 virgata Nutt.

\section{Parryella 3395}

756 I filifolia T. \& G.

7562 rotundata Wooton

\section{Amorpha $\mathbf{3 3 9 6}$}

7563 Californica Nutt.

7564 canescens Pursh

7565 fruticosa L.

7566 glabra Desf.

7567 herbacea Walt.

7568 hispidula Greene

7569 laevigata Nutt.

7570 nana Nutt.

$$
\text { (A. microphylla Pursh) }
$$

7571 paniculata T. \& G.

7572 subglabra (A. Gray) Heller

7573 virgata Small

\section{Eysenhardtia 3398}

7574 amorphoides H. B. K.

7575 orthocarpa (A. Gray) Wats. 7576 spinosa Engelm.

\section{Parosela $\mathbf{3 3 9 8}$} (Dalea)

7577 albiflora (A. Gray) Vail

7578 amoena (Wats.) Vail

7579 arborescens ('Torr.) Heller 

$75^{80}$ argyraea (A. Gray) Heller

$75^{81}$ aurea (Nutt.) Britton

$75^{82}$ brachystachys (A.Gray) Heller

7583 Californica (Wats.) Vail

7584 calycosa (A. Gray) Heller

$75^{8} 5$ Dalea (L.) Britton

(D. alopecuroides Willd.)

7586 Domingensis (DC.) Heller

$75^{87}$ elatior (A. Gray) Vail

$75^{88}$ Emoryi (A. Gray) Heller

$75^{89}$ enneandra (Nutt.) Britton

(D. laxiflora Pursh)

7590 filiformis (A. Gray) Heller

759I formosa (A. Gray) Vail

7592 Fremontii (Torr.) Vail

7593 frutescens (A. Gray) Vail

7594 Grayi Vail

7595 Greggii (A. Gray) Heller

7596 Hallii (A. Gray) Heller

7597 Jamesii ('T. \& G.) Vail

7598 Johnsoni (Wats.) Vail

7599 Kingii (Wats.) Heller

7600 lachnostachya (A.Gray) Heller

760 I lanata (Spreng.) Britton

7602 lasiathera (A. Gray) Heller

7603 Lemmoni (Parry) Heller

7604 Lumholtzii (Robinson\&Fern.)

(P. Arizonica Vail.) Vail

7605 mollis (Benth.) Heller

7606 nana (Torr.) Heller

7607 Neo-Mexicana (A.Gray) Heller

7608 Ordiae (A. Gray) Heller

7609 Parryi ('T. \& G.) Heller

76ro pogonathera (A. Gray) Vail

76 I 1 polyadenia ('Torr.) Heller

$76 \mathrm{I}_{2}$ subnuda (Wats.)

76r 3 polygonoides (A. Gray) Heller

76r 4 Pringlei (A. Gray) Heller

76 I 5 pulchella (Moricand) Heller

7616 ramosissima (Benth.) Heller

$76 \mathrm{I} 7$ scariosa (Wats.) Heller

76r 8 Schottii ('Torr.) Heller

76I9 scoparia (A. Gray) Heller

7620 spinosa (A. Gray) Heller

762 I Thompsonae Vail

7622 thyrsiflora (A. Gray) Vail

7623 Wheeleri Vail

7624 Wislizeni (A. Gray) Vail
7625 sessilis (A. Gray) Vail

7626 Wrightii (A. Gray) Vail

\section{Kuhnistera $\mathbf{3 3 9 9}$}

7627 pinnata (Walt.) Kuntze

(Petalostemon corymbosum Michx.)

\section{Petalostemon}

(3399)

7628 candidus Michx.

7629 carneus Michx.

7630 compactus (Spreng.) Swezy

(P. macrostachyus Torr.)

763 I decumbens Nutt.

7632 emarginatus Nutt.

7633 exilis A. Gray

7634 Feayi Chapm.

7635 flavescens Wats.

7636 foliosa A. Gray

7637 Gattingeri Heller

$763^{8}$ gracilis Nutt.

7639 griseus T. \& G.

7640 microphyllus ('T. \& G.) Heller

764I mollis Rydb.

7642 multiflorus Nutt.

7643 obovatus T. \& G.

7644 oligophyllus (Torr.) Rydb.

7645 ornatus Dougl.

7646 phleoides T. \& G.

7647 pulcherrimus Heller

7648 purpureus (Vent.) Rydb.

( $P$. violaceus Michx.)

7649 Reverchoni Wats.

7650 Sabinalis Wats.

765I Searlsiae A. Gray

7652 tenuifolius A. Gray

7653 tenuis (Coult.) Heller

7654 villosus Nutt.

\section{Brongniartia $\mathbf{3 4 0 3}$}

7655 minutifolia Wats.

\section{Cracca 340\%}

\section{(Tephrosia)}

7656 ambigua (M. A. Curtis) Kuntze 7657 angustissima(Shuttlw.)Kuntze 7658 chrysophylla (Pursh) Kuntze

7659 . Chapmani Vail

7660 cinerea (L.) Morong

7661 fiexuosa (Chapm.) Heller 

7662 Floridana Vail

7663 gracillima (Robinson) Heller 7664 hispidula (Michx.) Kuntze

7665 holosericea (Nutt.)

Britten \& Baker

7666 leiocarpa (A. Gray) Kuntze

7667 leucantha (H. B. K.) Kuntze

7668 Lindheimeri (A. Gray) Kuntze

7669 onobrychoides (Nutt.) Kuntze

7670 purpurea L.

767 I Rugelii (Shuttlw.) Heller

7672 Smallii Vail

7673 Virginiana L.

(T. spicata T. \& G.)

\section{Kraunhia $\mathbf{3 4 1 0}$}

(Wisteria)

7674 frutescens (L.) Greene

7675 macrostachys ('T. \& G.) Small

\section{Peteria 3418}

7676 scoparia A. Gray

7677 Thompsonae Wats.

\section{Robinia 34\%0}

7678 Boyntonii Ashe

7679 hispida L.

7680 Elliottii Chapm.

768I macrophylla DC.

7682 nana Ell.

7683 rosea Pursh

7684 Neo-Mexicana A. Gray

7685 Pseudacacia L.

7686 Decaisneana Carr.

7687 viscosa Vent.

\section{Olneya $\mathbf{3 4 2 6}$}

7688 Tesota A. Gray

\section{Coursetia $\mathbf{3 4 3 0}$}

7689 axillaris Coult. \& Rose

7690 glandulosa A. Gray

\section{Brittonamra $\mathbf{3 4 : 3 1}$}

(Cracca)

769r Edwardsii (A. Gray) Kuntze 7692 sericea (A. Gray) Kearney

\section{Sesban 3433}

(Sesbania)

7693 grandiflora Poir.
7694 macrocarpa Muh1.

7695 Thurberi Wats.

7696 vesicaria Ell.

\section{Daubentonia}

7697 longifolia (Cav.) DC.

\section{Glottidium (3433)}

7698 Floridanum (Willd.) DC.

7699 atrorubens Nash

\section{Astragalus $\mathbf{3 4 5 0}$}

7700 accidens Wats.

7701 accumbens Sheldon

7702 acerbus Sheldon

7703 adsurgens Pall.

7704 albatus Sheldon

7705 albens Greene

7706 allanaris Sheldon

7707 allochrous A. Gray

7708 alpinus $\mathrm{L}$.

7709 littoralis (Hook.) Sheldon

7710 amphioxys Wats.

77 II ampullarius Wats.

7712 Andersonii A. Gray

7713 angustus Jones

7714 anisus Jones

7715 Antiselli A. Gray

7716 apertus Sheldon

7717 apilosus Sheldon

(A. glaber Michx.)

7718 araneosus (Jones) Sheldon

7719 argillosus Jones

7720 argophyllus Nutt.

7721 Panguicensis Jones

7722 aridus A. Gray

7723 arietinus Jones

7724 stipularis Jones

7725 Arizonicus A. Gray

7726 arrectus A. Gray

7727 eremiticus Jones

7728 artemisiarum Jones

7729 Arthuri Jones

7730 artipes A. Gray

773 I asclepiadoides Jones

$773^{2}$ asymmetricus Sheldon

(A. leucophyllus 'T. \& G.)

7733 atratus Wats.

7734 arctus Sheldon 

7735 mensanus Jones

7736 atropubescens Coult. \& Fisher

7737 Austinae A. Gray

7738 Beckwithii T. \& G.

7739 Bernardinus Jones

7740 bicristatus A. Gray

774 I Bigelovii A. Gray

7742 bisulcatus (Hook.) A. Gray

7743 Bodini Sheldon

7744 Bolanderi A. Gray

7745 Brandegei Porter

7746 Brazoensis Buckley

7747 Breweri A. Gray

7748 brevicaulis Aven Nelson

7749 Californicus Greene

$775^{\circ}$ calycosus Torr.

775I campylophyllus Greene

$775^{2}$ Candelarius Sheldon

7753 exiguus Sheldon

7754 candicans Greene

7755 Candolleanus(H.B.K.)Sheldon

7756 canonis Jones

7757 Carolinianus L.

$$
\text { (A. Canadensis I.) }
$$

$775^{8}$ Casei A. Gray

7759 castanaeformis Wats.

7760 Catalinensis Nutt.

776r cerussatus Sheldon

7762 Chamaeleuce A. Gray

7763 cibarius Sheldon

7764 cicadae Jones

7765 laccoliticus Jones

7766 Clevelandi Greene

7767 Cobrensis A. Gray

7768 coccineus Brandegee

7769 collinus Dougl.

7770 Coltoni Jones

777 I Moabensis Jones

7772 confertiflorus A. Gray

7773 Congdoni Wats.

7774 conjunctus Wats.

7775 oxytropidoides Jones

7776 consectus Sheldon

7777 convallarius Greene

$$
\text { (A. campestris A. Gray) }
$$

7778 Coulteri Benth.

7779 crassicarpus Nutt. (A. caryocarpus $\mathrm{Ker}$ )
7780 crescenticarpus Sheldon

778 I crotalariae (Benth.) A. Gray

7782 curtipes A. Gray

7783 Cusickii A. Gray

7784 cuspidocarpus Sheldon

7785 cyaneus A. Gray

7786 cymatodes Greene

7787 cymboides Jones

7788 debilis A. Gray

7789 decumbens A. Gray

7790 desperatus Jones

7791 diaphanus Dougl.

7792 didymocarpus H. \& A.

7793 diphysus A. Gray

7794 dispermus A. Gray

7795 distortus T. \& G.

7796 diurnus Wats.

7797 diversifolius A. Gray

7798 Dodgianus Jones

7799 dorycnoides Dougl.

7800 Douglasii (T. \& G.) A. Gray

$780 \mathrm{o}$ glaberrimus Jones

7802 drepanolobus A. Gray

7803 Drummondii Dougl.

7804 Eastwoodiae Jones

7805 elatiocarpus Sheldon

(A. lotiflorus var. brachycarpus

A. Gray)

7806 elegans (Hook.) Sheldon

7807 curtiflorus Rydb.

7808 Elmeri Greene

7809 Engelmanni Sheldon

7810 ensiformis Jones

78 I I episcopus Wats.

7812 eremicus Sheldon

78 I 3 eremiticus Sheldon

7814 Eurekensis Jones

78r 5 exilifolius Aven Nelson

7816 famelicus Sheldon

7817 Feensis Jones

781 8 Fendleri A. Gray

78 r 9 filipes Torr.

7820 flaviflorus (Kuntze) Sheldon

$$
\text { (L. Aavus Nutt.) }
$$

782 I flexuosus (Hook.) Dougl.

7822 foliolosus (A. Gray) Sheldon

7823 Forwoodii Wats.

7824 Franciscanus Sheldon 

7825 Fremonti Torr. (A. ineptus A. Gray)

7826 Gambellianus Sheldon

7827 Geyeri A. Gray

7828 Gibbsii Kellogg

7829 Gilensis Greene

7830 gilviflorus Sheldon

783 I glareosus Dougl.

7832 gracilentus A. Gray

7833 gracilis Nutt.

7834 grallator Wats.

7835 Grayi Parry

7836 Greenei A. Gray

7837 griseopubescens Sheldon

7838 Hallii A. Gray

7839 Hartwegi Benth.

7840 Hasseanus Sheldon

784 I Haydenianus A. Gray

7842 Nevadensis Jones

7843 Hoodianus Howell

7844 Hookerianus ('T. \& G.) A. Gray

7845 Hornii A. Gray

7846 minutiflorus Jones

7847 Hosackiae Greene

7848 Howellii A. Gray

7849 humillimus A. Gray

7850 humistratus A. Gray

$785 \mathrm{I}$ tenerrimus A. Gray

7852 hyalinus Jones

7853 hypoglottis L.

7854 bracteosus Osterhout

7855 hypoxylus Wats.

7856 Ibapensus Jones

7857 inflexus Dougl.

$785^{8}$ iodanthus Wats.

7859 insularis Kellogg

7860 intonsus Sheldon

786I inversus Jones

7862 Inyoensis Sheldon

7863 jejunus A. Gray

7864 Jepsoni Sheldon

7865 junciformis Aven Nelson

7866 Kelseyi Rydb.

7867 lancearius A. Gray

7868 lanocarpus Sheldon

7869 latus Jones

7870 Laynae Greene

$787 \mathrm{I}$ lectulus Wats.
7872 Leibergii Jones

7873 Lemmoni A. Gray

7874 lentiformis A. Gray

7875 lentiginosus Dougl.

7876 Borreyanus Jones

7877 chartaceus Jones

7878 nigricalycis Jones

7879 Yuccanus Jones

7880 leptaleus A. Gray

788I leptocarpus T. \& G.

7882 leucolobus Jones

7883 lencopsis Torr.

7884 curtus Sheldon

7885 limatus Sheldon

7886 Lindheimeri A. Gray

7887 lingulatus Sheldon

7888 lonchocarpus Torr.

7889 lotiflorus Hook.

$7890 \quad$ Nebraskensis Bates

7891 Lyallii A. Gray

7892 MacDougali Sheldon

7893 Macounii Rydb.

7894 inacrodon (H. \& A.) A. Gray

7895 malacus A. Gray

7896 Malheurensis Heller

7897 Matthewsii Wats.

7898 megacarpus (Nutt.) A. Gray

7899 caulescens Jones

7900 prodigus Sheldon

(A. megacarpus var. Parryi A. Gray)

790 I Menziesii A. Gray

7902 Mexicanus A. DC.

7903 microlobus A. Gray

7904 Miguelensis Greene

7905 misellus Wats.

7906 miser Dougl.

7907 Missouriensis Nutt.

7908 Moencoppensis Jones

7909 Mogollonicus Greene

7910 Mohavensis Wats.

79I I Mokiacensis A. Gray

7912 mollissimus Torr.

7913 Mortoni Nutt.

7914 Mulfordae Jones

7915 multiflorus A. Gray

7916 Musiniensis Jones

7917 neglectus (T. \& G.) Sheldon

(A. Cooperi A. Gray) 

7918 Nevinii A. Gray

7919 Newberryi A. Gray

7920 castoreus Jones

792 I Nuttallianus DC.

7922 canescens T. \& G.

7923 enneajugus Jones

7924 leptocarpoides Jones

7925 quadrilateralis Jones

7926 trichocarpus T. \& G.

7927 obcordatus Ell.

7928 oblatus Sheldon

$$
\text { (A. mudus Wats.) }
$$

7929 obscurus Wats.

7930 occidentalis (Wats.) Jones

793 I oocalycis Jones

7932 oocarpus A. Gray

7933 oophorus Wats.

7934 Oreganus Nutt.

7935 oxyphysus A. Gray

7936 pachypus Greene

7937 Pacificus Sheldon

7938 palans Jones

7939 Palmeri A. Gray

7940 Palousensis Piper

794I Panamintensis Sheldon

7942 Parishii A. Gray

7943 Parryi A. Gray

7944 Pattersoni. A. Gray

7945 pauperculus Greene

7946 Peabodianus Jones

7947 pectinatus (Hook.) Dougl.

7948 pephragmenus Jones

7949 Phoenicis Jones

7950 Pinonis Jones

795 I Plattensis Nutt.

7952 playanus Jones

7953 polaris Benth.

7954 porrectus Wats.

7955 praelongus Sheldon

7956 Preusii A. Gray

7957 arctus Sheldon

$795^{8}$ laxispicatus Sheldon

7959 prunifer Rydb.

7960 pruniformis Jones

7961 pterocarpus Wats.

7962 pubentissimus T. \& G.

7963 Pulsiferae A. Gray

7964 Purshii Dougl.
7965 longilobus Jones

7966 tinctus Jones

7967 pycnostachys A. Gray

7968 racemosus Pursh

7969 longisetus Jones

7970 rasus Sheldon

797I Rattani A. Gray

7972 reflexus 'T. \& G.

7973 remulcus Jones

7974 reventoides Jones

7975 reventus A. Gray

7976 Canbyi Jones

7977 Reverchoni A. Gray

7978 Robbinsii (Oakes) A. Gray

7979 Rothrockii Sheldon

7980 Rusbyi Greene

7981 longissimus Jones

7982 sabulonis A. Gray

7983 sabulosus Jones

7984 salinus Howell

7985 salmonus Jones

7986 scaposus A. Gray

7987 sclerocarpus A. Gray

7988 scobinatulus Sheldon

7989 scophioides (Jones) Rydb.

7990 scopulorum Porter

799I secundus Michx.

$$
\text { (A. Bakeri Eggleston) }
$$

7992 'Serenoi Sheldon

7993 sericoleucus A. Gray

7994 serpens Jones

7995 sesquiflorus Wats.

7996 Shockleyi Jones

7997 Shortianus Nutt.

7998 Sileranus Jones

7999 cariacus Jones

8000 simplicifolius A. Gray

800 I Sonneanus Greene

8002 Sonorae A. Gray

8003 sophoroides Jones

8004 Spaldingii A. Gray

8005 sparsiflorus A. Gray

8006 majusculus A. Gray

8007 speirocarpus A. Gray

8008 curvicarpus Sheldon

8009 straturensis Jones

8 oro striatiflorus Jones

8oI I strigosus (Kellogg) Sheldon 

80I 2 subcinereus A. Gray

8013 succumbens Dougl.

8or4 Suksdorfii Howell

8015 supervacanus Greene

8016 suturalis Sheldon

8017 tegetarius Wats.

8018 implexus Canby

8019 rotundus Jones

8020 Tennesseensis A. Gray

802 I tephrodes A. Gray

8022 terminalis Wats.

8023 tetrapterus A. Gray

8024 Texanus Sheldon

8025 Thompsonae Wats. (A. syrticolus Sheldon)

8026 Thurberi A. Gray

8027 Toanus Jcnes

8028 Traskiae Eastwood

8029 tricarinatus A. Gray

8030 trichopodus A. Gray

8031 tridactylicus A. Gray

8032 triquetrus A. Gray

8033 troglodytus Wats.

8034 Tweedyi Canby

8035 umbraticus Sheldon

8036 ursinus (Torr.) A. Gray

8037 Utahensis T. \& G.

8038 vaccarum A. Gray

8039 Vaseyi Wats.

8040 ventorum A. Gray

804 I vespertinus Sheldon

8042 villosus Michx.

8043 Virgineus Sheldon

8044 virgultulus Sheldon

8045 Wardii A. Gray

8046 Watsoni Sheldon

$$
\text { (A. Hendersoni Wats.) }
$$

8047 Watsonianus (Kuntze)Sheldon (A. eriocarpus Wats.)

8048 Webberi A. Gray

8049 Wetherilli Jones

8050 Whitneyi A. Gray.

805I Wilsoni Greene

8052 Wingatanus Wats.

8053 Wootoni Sheldon

8054 Wrightii A. Gray

8055 Zionis Jones

\section{Phaca (3450)}

(Astragalus in part)

8056 Americana (Hook.) Rydb.

8057 inepta.(A. Gray) Rydb.

8058 longifolia (Pursh) Nutt.

8059 microcystis (A. Gray) Rydb.

8060 neglecta T. \& G.

806I platytropis (A. Gray) Rydb.

\section{Homalobus (3450)}

8062 aboriginorum(Richards.)Rydb. 8063 aculeatus (Aven Nelson) Rydb. 8064 Bourgovii (A. Gray) Rydb.

8065 caespitosus Nutt.

8066 campestris Nutt.

8067 glabriusculus (A. Gray) Rydb.

8068 hylophilus Rydb.

8069 junceus Nutt.

8070 montanus (Nutt.) Britton

(A. Kentrophyta A. Gray)

807 I Palliseri (A. Gray) Rydb.

8072 serotinus (A. Gray) Rydb.

8073 spatiosus (Sheldon) Heller

8074 stenophyllus ('T. \& G.) Rydb.

8075 tenellus (Pursh) Britton

(A. multiflorus A. Gray)

8076 tenuifolius Nutt.

8077 vexilliflexus (Sheldon) Rydb.

(A. panciflorus Hook.)

Orophaca (3450)

(Astragalus in part)

8078 caespitosa (Nutt.) Britton

8079 sericea (Nutt.) Britton

\section{Aragallus 3451}

(Oxytropis; Spiesia)

8080 acutirostris (Wats.) Heller

808I alpicola Rydb.

8082 arcticus (R. Br.) Greene

8083 argophyllus Rydb.

8084 Bellii (Britton) Greene

8085 Besseyi Rydb.

(Oxytropis argentata Pursh)

8086 Bigelovii (A. Gray) Greene 8087 Blankinshipii Aven Nelson 8088 campestris caeruleus (Koch) 

8089 caudatus Greene

8090 collinus Aven Nelson

8ogr deflexus (Pall.) Heller

8092 dispar Aven Nelson

8093 foliolosus (Hook.) Rydb.

8094 gracilis Aven Nelson

8095 inflatus (Hook.) Aven Nelson

8096 involutus Aven Nelson

8097 Johannensis (Fernald) Heller

8098 lagopus (Nutt.) Greene

8099 Lamberti (Pursh) Greene

8roo leucanthus (Pall.) Greene

8ror Mertensianus ('Turcz.) Greene

$8 \mathrm{ro} 2$ monticola (A. Gray) Greene

8103 multiceps (Nutt.) Heller

8104 minor (A. Gray)

8 Io5 nanus (Nutt.) Greene Aven Nelson

8ro6 nigrescens (Pall.) Heller

8Io7 nothoxys (A. Gray) Heller (Astragalus nothoxys A. Gray)

8108 oreophyllus (A. Gray) Greene 8109 Parryi (A. Gray) Greene

8IIo pinetorum Heller

8III Richardsonii (Hook.) Greene

8 I 2 sericeus (Nutt.) Greene

8II3 spicatus (Hook.) Rydb.

(A. albiflorus Aven Nelson)

8II4 splendens (Dougl.) Greene

8 II5 viscidulus Rydb.

8II6 depressus Rydb.

8 II 7 viscidus (Nutt.) Greene

\section{Glyeyrrhiza $\mathbf{3 4 5 3}$}

8II8 glutinosa Nutt.

8Ir 9 lepidota Nutt.

\section{Coronilla $\mathbf{3 4 5 8}$}

8120 varia $\mathrm{L}$.

\section{Onobrychis}

8I2I Onobrychis (L.) Rydb. (O. sativa Lam.)

\section{Hedysarum $\mathbf{3 4 6 2}$}

8122 Americanum (Michx.) Britton (H. boreale Nutt.)

8 23 carnosulum Greene

$8 \mathrm{r} 24$ cinerascens Rydb. (H. canescens Nutt.)
8I 25 lancifolium Rydb.

8i26 leucanthum Greene

81 27 Mackenzii Richards.

81 28 marginatum Greene

8r 29 occidentale Greene

8I 30 sulphurescens Rydb.

(H. Alavescens Coult. \& Fisher)

Nissolia $\mathbf{3 4 6 8}$

8I 3I Schottii ('Torr.) A. Gray

\section{Chaetocalyx}

81 32 Wislizeni A. Gray

Aeschynomene $\mathbf{3 4 7 6}$

8I 33 Virginica (L.) B. S. P. (A. hispida Willd.)

8I 34 viscidula Michx.

\section{Chapmania $\mathbf{3 4 8 3}$}

8I35 Floridana T. \& G.

\section{Stylosanthes $\mathbf{3 4 8 4}$}

8 I 36 biflora (L.) B. S. P.

$$
\text { (S. elatior Sw.) }
$$

8I 37 hispidissima (Michx.) Pollard \& Ball

8 I 38 riparia Kearney

\section{Zornia $\mathbf{3 4 8 6}$}

8I 39 bracteata (Walt.) Gmel (Z. tetraphylla Michx.)

\section{Meibomia $\mathbf{3 4 8 9}$}

(Desmodium)

8r4o arenicola Vai]

(D. lineatum DC.)

8I4I Arizonica (Wats.) Vail

$8 \mathrm{I} 42$ batocaulis (A. Gray) Kuntze

8I 43 Bigelovii (A. Gray) Kuntze

8144 bracteosa (Michx.) Kuntze (D. cuspidatum Hook.)

8I45 Canadensis (L.) Kuntze

8I46 canescens (L.) Kuntze

8I 47 hirsuta (Hook.) Vail

8I48 cinerascens (A. Gray) Kuntze

8I49 Dillenii (Darl.) Kuntze

8I 50 glabella (Michx.) Kuntze (D. humifusum Beck)

8I 5I Grahami (A. Gray) Kuntze 

8152 graminea (A. Gray) Kuntze 8I 53 grandiflora (Walt.) Kuntze (D. acuminatum DC.)

8I 54 Illinoensis (A. Gray) Kuntze 8I 55 incana (DC.) Vail

8I 56 laevigata (Nutt.) Kuntze

8157 Lindheimeri Vail

8I 58 longifolia (T. \& G.) Vail

8I 59 Marylandica (L.) Kuntze

8160 Michanxii Vail

(D. rotundifolium $\mathrm{DC}$.)

8r6r Neo-Mexicana(A.Gray)Kuntze 8rg8 Brownei (Pers.) Kuntze

8 r62 nudiflora (L.) Kuntze

8163 obtusa (Muh1.) Vail

$$
\text { (D. ciliare DC.) }
$$

8164 ochroleuca (M. A. Curtis)

8 I65 paniculata (L.) Kuntze

Kuntze

8166 Chapmani Britton

8167 pubescens ('T. \& G.) Vail

8I68 pauciflora (Nutt.) Kuntze

8I69 psilocarpa (A. Gray) Kuntze

81 7o rhombifolia (E11.) Vail (D. Floridanum Chapm.)

8I 7 r rigida (Ell.) Kuntze

$8 \mathrm{r} 72$ sessilifolia (Torr.) Kuntze

$8 \mathrm{I} 73$ spiralis (DC.) Kuntze

8r 74 stricta (Pursh) Kuntze

8I 75 tenuifolia ('T. \& G.) Kuntze

8176 triflora (L.) Kuntze

8I 77 tortuosa (Sw.) Kuntze

81 78 Tweedyi (Britton) Vail

8I79 uncinata (Jacq.) Kuntze

8I 80 viridiflora (L.) Kuntze

8I8I Wrightii (A. Gray) Kuntze

\section{Lespedeza 3501}

8 I82 angustifolia (Pursh) Ell.

8 I8 3 capitata Michx.

8I84 longifolia (DC.) T. \& G.

8I85 sericea Hook.

8I 86 frutescens (L.) Britton

(L. reticulata Wats. not Pers. and $L$. stuvei var. intermedia 8 I87 hirta (L.) Ell. (L. polystachya Michx.) 8188 oblongifolia Britton 8r89 leptostachya Engelm. 8rgo Nuttallii Darl.

8I9I procumbens Michx.

8 I 92 repens (L.) Bart.

8193 striata ('Thunb.) H. \& A.

8I94 Stuvei Nutt.

8195 neglecta Britton

8I96 violacea (L.) Pers.

8197 Virginica (L.) Britton

\section{Dalbergia 350\%}

(Ecastophyllum)

\section{Ichthyomethia $\mathbf{3 5 1 9}$}

$$
\text { (Piscidia) }
$$

8I 99 piscipula (L:) Kuntze (Piscidia Erythrina L.)

\section{Cicer 3529}

8200 arietinum L.

\section{Vicia 3530}

8201 acutifolia Ell.

8202 Americana Muhl.

8203 angustifolia Roth.

8204 caespitosa Aven Nelson

8205 Californica Greene

8206 Caroliniana Walt.

8207 Cracca L.

8208 exigua Nutt.

8209 Floridana Wats.

82 Io gigantea Hook.

82 II Hassei Wats.

8212 hirsuta (L.) Koch

82I3 Hugeri Small

82I4 Leavenworthii T. \& G.

82 I5 leucophaea Greene

8216 linearis (Nutt.) Greene

8217 Ludoviciana Nutt.

8218 micrantha Nutt.

82 I9 pulchella H. B. K.

8220 Reverchoni Wats.

822 I sativa $\mathrm{L}$.

8222 semicincta Greene

8223 Sepium L.

8224 tetrasperma (L.) Moench.

8225 Thurberi Wats.

8226 truncata Nutt. 



\section{Ervum (3530)}

8227 Lens L.

\section{Lathyrus $\mathbf{3 5 3 2}$}

8228 Alefeldi White

8229 Arizonicus Britton

8230 bijugatus White

8231 Bolanderi Wats.

8232 Californicus Wats.

8233 coriaceus White

8234 Cusickii Wats.

8235 decaphyllus Pursh

(L. polymorphus Nutt.)

8236 graminifolius (Wats.) White

8237 Jepsoni Greene

8238 laetiflorus Greene

8239 lanceolatus Howell

8240 littoralis (Nutt.) Endl.

824I maritimus (L.) Bigel.

8242 Aleuticus Greene

8243 myrtifolius Muhl.

8244 macranthus White

8245 Nuttallii Wats.

8246 obovatus (Torr.) White (L. Nevadensis Wats.)

8247 stipulaceus White

8248 ochroleucus Hook.

8249 Oregonensis White

8250 ornatus Nutt.

825 I palustris L.

8252 parvifolius Wats.

8253 pauciflorus Fernald

8254 polyphyllus Nutt.

8255 pratensis L.

8256 puberulus White

8257 pusillus E11.

\section{(L. Engelmanni Bisch.)}

$825^{8}$ rigidus White

8259 Sandbergii (White) Howell

8260 Schaffneri Rydb.

(L. parviflorus Wats. not Roth)

826I splendens Kellogg

8262 sulphureus Brewer

8263 Torreyi A. Gray

8264 tenellus Wiegand

8265 Utahensis Jones

8266 venosus Muhl.

8267 vestitus Nutt.
8268 violaceus Greene

8269 Barbarae White

8270 Watsoni White

(L. venosus var. Californicus Wats.)

Clitoria 3538

827 I Mariana L.

\section{Bradburya $\mathbf{3 5 3 9}$}

(Centrosema)

8272 Plumieri (Turp.) Kuntze

8273 Virginiana (L.) Kuntze

\section{Falcata 3541}

(Amphicarpaea)

8274 comosa (L.) Kuntze (A. monoica Nutt.)

8275 Pitcheri (T. \& G.) Kuntze

\section{Cologania (3541)}

8276 angustifolia Kunth.

8277 Lemmoni A. Gray

8278 longifolia A. Gray

8279 pulchella H. B. K.

\section{Erythrina $\mathbf{3 5 5 0}$}

8280 Corallodendron L.

828I flabelliformis Kearney (E. coralloides Moc. \& Sesse)

8282 herbacea L.

\section{Apios $\mathbf{3 5 5 3}$}

8283 Apios (L.) MacM. (A. tuberosa Moench)

8284 Priceana Robinson

\section{Galactia $\mathbf{3 5 6 1}$}

8285 brachypoda T. \& G.

8286 canescens (Scheele) Benth.

8287 Cubensis H. B. K.

8288 Elliottii Nutt.

8289 erecta (Walt.) Vail. (G. sessiliflora T. \& G.)

8290 fasciculata Vail

829I Floridana 'T. \& G.

8292 Grayi Vail

8293. heterophylla (Gillies) Vail

(G. marginalis Benth.)

8294 mollis Michx. 

8295 regularis (L.) B. S. P. (G. glabella Michx.)

8296 Texana (Scheele) A. Gray

8297 volubilis (L.) Britton (G. pilosa Ell.)

8298 intermedia Vail

8299 Mississippiensis Vail

8300 Wrightii A. Gray

\section{Dioclea $\mathbf{3 5 6 5}$}

83or Boykinii A. Gray

Canavalia $\mathbf{3 5 6 8}$

8302 altissina MacF.

8303 obtusifolia DC.

Dolicholus $35 \% 4$

(Rhynchosia)

8304 Americanus (Mill.) Vail

(R. menispermoides DC.)

8305 angustifolius(Engelm.)Kuntze

8306 cinereus (Nash) Vail

8307 Drummondii Vail

8308 erectus (Walt.) Vail

8309 intermedius ('T. \& G.) Vail

83I0 latifolius (Nutt.) Vail

83 i L Lewtoni Vail

83 I2 Michauxii Vail

(R. menispermoides Chapm. not DC.)

83 I 3 minimus (L.) Medic

83 I 4 mollissimus (E1l.) Vail.

83 I5 parvifolius (DC.) Vail

83 I6 simplicifolius (Walt.) Vail

8317 Swartzii Vail

(R. Caribaea Chapm. not DC.)

83I8 Texanus ('T. \& G.) Vail

83 I9 tomentosus (L.) Vail

8320 undulatus Vail

832 I Torreyi Vail

\section{Phaseolus 3578}

8322 acutiflorus A. Gray

8323 angustissimus A. Gray

8324 atropurpureus Moc.

8325 macropoides A. Gray

8326 polystachys (L.) B. S. P. (P. perennis Walt.)

8327 retusus Benth.

8328 rotundifolius A. Gray
8329 sinuatus Nutt.

8330 smilacifolius Pollard

8331 Wrightii A. Gray

Strophostyles (35\%8)

(Phaseolus in part)

8332 helveola (L.) Britton

(S. angulosa Ell.)

8333 Missouriensis (Wats.)

Britton

8334 pauciflora (Benth.) Wats.

8335 umbellata (Muhl.) Britton

(S. peduncularis Ell.)

Vigna 3581

8336 repens (L.) Kuntze ( $V$. luteola Benth.)

8337 Sinensis (L.) Endl.

\section{Dolichos $\mathbf{3 5 8 3}$}

$833^{8}$ Lablab L.

\section{GERA NIACEAE}

\section{Geranium $\mathbf{3 5 8 5}$}

8339 atropurpureum Heller

8340 Bicknellii Britton

834I caespitosum James

8342 Carolinianum L.

8343 longipes Wats.

8344 columbinum L.

8345 dissectum L.

8346 erianthum DC.

8347 Fremontii Torr.

8348 Hernandezii DC.

8349 incisum Nutt.

835 L Langloisii Greene

835 I maculatum L.

8352 Mexicanum H. B. K.

8353 molle L.

8354 Oreganum Howell

8355 Parryi (Engelm.) Heller

8356 parviflorum Willd.

8357 pentagynum Engelm.

$835^{8}$ pilosum Forst. f.

8359 pusillum L.

8360 Richardsoni F. \& M.

836 I albiflorum Cockerell

8362 Robertianum L.

8363 rotundifolium L. 

8364 Sibiricum L.

8365 Texanum ('Trelease) Heller

\section{Erodium 3588}

8366 Botrys Bertol.

8367 Californicum Greene

8368 ciconium Willd.

8369 cicutarium (L.) L'Her.

8370 macrophyllum H. \& A.

837 I malacoides Willd.

8372 moschatum Willd.

8373 Texanum A. Gray

\section{Pelargonium $\mathbf{3 5 8 9}$}

8374 anceps L'Her.

\section{OXALIDACEAE}

\section{Oxalis 359 '}

8375 Acetosella L.

8376 Berlandieri Torr.

8377 Bushii Small

8378 corniculata L.

8379 cymosa. Small

8380 decaphylla H. B. K.

838 I dichondraefolia A. Gray

8382 divergens Benth.

8383 amplifolia Trelease

8384 Drummondii A. Gray

8385 filipes Small

8386 grandis Small

8387 hirsuticanlis Small

8388 macrantha (Trelease) Small

8389 Martiana Zucc.

8390 Oregana Nutt.

839 I pilosa Nutt.

8392 Priceae Small

8393 recurva Ell.

8394 stricta L.

8395 Suksdorfii Trelease

8396 trilliifolia Hook.

8397 violacea L.

8398 Wrightii A. Gray

TROPAEOLACEAE

Tropaeolum $\mathbf{3 6 0 0}$

8399 majus L.

\section{LINACEAE}

Linum $\mathbf{3 6 0 3}$

8400 adenophyllum A. Gray
840 I aristatum Engelm.

8402 subteres Trelease

8403 australe Heller

8404 Berlandieri Hook.

8405 Plotzii Trelease

8406 Breweri A. Gray

8407 Californicum Benth.

8408 confertum A. Gray

8409 catharticum L.

8410 Clevelandi Greene

84I I congestum A. Gray

84I 2 digynum A. Gray

84 I 3 drymarioides Curran

84r 4 Floridanum (Planch.) Trelease

84I5 Greggii Engelm.

8416 humile Mill.

8417 Kingii Wats.

8418 pinetorum Jones

8419 Lewisii Pursh

8420 albiflorum Cockerell

842 I medium (Planch.) Britton

8422 micranthum A. Gray

8423 multicaule Hook.

8424 Neo-Mexicanum Greene

8425 puberulum (Engelm.) Heller

8426 rigidum Pursh

8427 rupestre Engelm.

8428 spergulinum A. Gray

8429 striatum Walt.

8430 sulcatum Riddell

84.3 I usitatissimum I.

8432 vernale Wooton

8433 Virginianum L.

\section{MALPIGHIACEAE}

Janusia $\mathbf{3 6 4 8}$

8434 gracilis A. Gray

Aspicarpa $\mathbf{3 6 5 0}$

8435 hyssopifolia A. Gray

8436 longipes A. Gray

Thryallis $\mathbf{3 6 6 1}$

(Galphimia)

8437 angustifolia (Benth.) Kuntze

$843^{8}$ oblongifolia (A. Gray) Vail

\section{Malpighia $\mathbf{3 6 6 8}$}

8439 glabra L. 

Byrsonimia 36\%1

8440 lucida (Sw.) H. B. K.

\section{ZYGOPHYLLACEAE}

Fagonia $36 \% 5$

844I Californica Benth.

8442 glutinosa Vail

\section{Zygophyllum 367g}

8443 fabago I.

Guajacum $\mathbf{3 6 \% 9}$

8444 sanctum L.

Porliera $\mathbf{3 6 8 0}$

8445 angustifolia (Engelm.) A.Gray

Covillea $\mathbf{3 6 8 3}$

(Larrea)

8446 divaricata (Cav.) Vail

8447 tridentata (DC.) Vail

(Larrea Mexicana Moric.)

Tribulus $\mathbf{3 6 8 6}$

8448 cistoides L.

8449 terrestris L.

Kallstroemia $\mathbf{3 6 8}$ \%

8450 brachystylis Vail

845 I Californica (Wats.) Vail

8452 grandiflora Torr.

8453 Arizonica Cockerell

8454 maxima (L.) T. \& G.

Peganum $\mathbf{3 6 9 2}$

8455 Mexicanum A. Gray

\section{RUTACEAE}

Zanthoxylum 3\%01

8456 Americanum Mill.

8457 Clava-Herculis L.

$845^{8}$ fruticosum A. Gray

8459 coriaceum A. Rich.

8460 cribrosum Spreng.

846I Fagara (L.) Sargent

$$
\text { (Z. Pterota H. B. K.) }
$$

8462 flavum Vahl.

8463 Texanum Buckley

Choisya 3\%1\%

8464 dumosa A. Gray
Astrophyllum $\mathbf{3 7 1 8}$

8465 dumosum Torr.

Thamnosma $\mathbf{3 7 \% 5}$

8466 montanum Torr.

8467 Texanum (A. Gray) Torr.

Cneoridium 3\%?6

8468 dumosum Hook. f.

Helietta 3\%

8469 parvifolia Benth.

\section{Ptelea 37\%9}

8470 angustifolia Benth.

847 I Baldwinii T. \& G.

8472 parvifolia A. Gray

8473 crenulata Greene

8474 mollis M. A. Curtis

8475 rhombifolia Heller

8476 trifoliata L.

\section{Amyris 3791}

8477 balsamifera L.

8478 elemifera L.

8479 maritima Jacq.

8480 parvifolia A. Gray

Citrus $380 \%$

$848 \mathrm{I}$ aurantiaca Bigaradia Brandis

SIMAROUBACEAE

Suriana ' 3808

8482 maritima L.

Simarouba $\mathbf{3 8 1 3}$

8483 glauca DC.

Castela 3820

8484 Nicholsoni Hook.

Holacantha 38\%1

8485 Emoryi A. Gray

Ailanthus 38\%6

8486 glandulosus Desf.

Picramnia $\mathbf{3 8 3 : 3}$

8487 pentandra Sw. 

BURSERACEAE

\section{Bursera}

8488 microphylla A. Gray

8489 Simaruba (L.) Sargent (B. gummifera Jacq.)

\section{MELIACEAE}

\section{Swietenia}

8490 Mahogoni L.

\section{Melia}

849r Azederach I.

\section{POLYGALACEAE}

\section{Polygala}

8492 acanthoclada A. Gray

8493 intricata Eastwood 8494 alba Nutt.

8495 suspecta Wats.

8496 ambigua Nutt.

8497 Arizonica Chodat

8498 Baldwinii Nutt.

8499 Boykini Nutt.

8500 brevifolia Nutt.

850 I Californica Nutt.

8502 Chapmani T. \& G.

8503 cornuta Kellogg

8504 cruciata L.

8505 cucullata Benth.

$8506^{\circ}$ Curtissii A. Gray

8507 cymosa Walt.

8508 grandiflora Walt.

8509 angustifolia T. \& G.

8510 canescens Shuttlw.

85II hemipterocarpa A. Gray

85I 2 Hookeri T. \& G.

85 I 3 incarnata $\mathrm{L}$.

85I4 leptocaulis T. \& G.

85 I5 leptostachys Shuttlw.

8516 Lewtonii Small

85I7 Lindheimeri A. Gray

85I 8 lonchophylla Greene

8519 lutea L.

8520 macradenia A. Gray

852 I Mariana Mill.

(P. fastigiata Nutt.)

8522 nana (Michx.) DC.
8523 Nuttallii DC.

8524 ovatifolia A. Gray.

8525 paludosa St. Hil.

8526 paucifolia Willd.

8527 polygama Walt.

8528 praetervisa Chodat

8529 puberula A. Gray

8530 ramosa Ell.

853 I Reynoldsae Chapm.

8532 Rugelii Shuttlw.

8533 Rusbyi Greene

8534 scoparia H. B. K.

8535 multicaulis A. Gray

8536 Senega L.

8537 latifolia T. \& G.

8538 setacea Michx.

8539 subspinosa Wats.

8540 Texensis Robinson

854 I Tweedyi Britton

8542 verticillata $L$.

8543 viridescens $\mathrm{L}$. ( $P$. sainguinea L.)

\section{Monnina}

8544 Wrightii A. Gray

\section{EUPHORBIACEAE}

Andrachne

8545 phyllanthoides (Nutt.)

Muell. Arg.

8546 Roemeriana Muell. Arg.

\section{Phyllanthus}

8547 abnormis Baill.

8548 Avicularia Small

8549 Carolinensis Walt.

$855^{\circ}$ Niruri L.

$855^{1}$ polygonoides Spreng.

Reverchonia

8552 arenaria A. Gray

\section{Drypetes}

8553 glauca Vahl.

8554 Keyensis Urban

8555 lateriflora (Sw.) Urban (D. crocea Poit.)

\section{Croton}

8556 Alabamensis E. A. Smith 

8557 balsamiferus Willd.

$855^{8}$ betulinus Vahl.

8559 Californicus Muell. Arg.

8560 major Wats.

856I capitatus Michx.

8562 Cortesianus H. B. K.

8563 corymbulosus Engelm.

8564 Elliottii Chapm.

8565 fruticulosus Torr.

8566 fuscescens Muell. Arg.

8567 pallescens Muell. Arg.

8568 glandulosus L.

8569 angustifolius Muell. Arg.

8570 septentrionalis Muell. Arg.

857 I leucophyllus Muell. Arg.

8572 Lindheimerianus Scheele

8573 linearis Jacq.

8574 longipes Jones

8575 maritimus Walt.

8576 monanthogynus Michx.

8577 Muelleri Coult.

8578 Neo-Mexicanus Muell. Arg.

8579 punctatus Jacq. (C. argyranthemus Michx.)

8580 Sonorae Torr.

858I suaveolens Torr.

8582 oblongifolius Torr.

$85^{8} 3$ tenuis Wats.

8584 Texensis (Klotzsch.) Muell. Arg.

8585 Torreyanus Muell. Arg.

\section{Crotonopsis}

8586 linearis Michx.

8587 spinosa Nash

\section{Eremocarpus}

8588 setigerus Benth.

\section{Ditaxis}

(Argyrothamnia)

8589 aphoroides (Muell. Arg.) Pax

8590 Blodgettii ('Torr.) Pax

859I humilis (Engelm. \& Gray) Pax

8592 laevis (Muell. Arg.) Heller

8593 mercurialina (Nutt.) Coult.

8594 Neo-Mexicana (Muell. Arg.)

Heller 8626 angustiloba Muell. Arg.

8595 pilosissima(Muell.Arg.) Heller 8627 Carthaginensis Muell. Arg.

8596 sericophylla (A. Gray) Heller

8597 serrata (Muell. Arg.) Heller

\section{Mercurialis}

8598 annuua L.

\section{Bernardia}

8599 myricaefolia Wats.

\section{Ricinella}

8600 Vaseyi (Coult.) Coult. \& Fisher

\section{Acalypha}

860r Californica Benth.

8602 corchorifolia Willd.

8603 gracilens A. Gray

8604 hederacea Torr.

8605 Lindheimeri Muell. Arg.

8606 Neo-Mexicana Muell. Arg.

8607 ostryaefolia Ridd.

8608 radians Torr.

8609 Virginica L.

\section{Tragia}

86ro laciniata Muell. Arg.

86 I m macrocarpa Willd.

86I 2 nepetaefolia Cav.

8613 ramosa Torr.

\section{I4 urens L.}

(T. stylaris Muell. Arg.)

86 5 urticaefolia Willd.

\section{Ricinus}

86I6 communis L.

\section{Jatropha}

86I 7 angustidens Muell. Arg.

86I 8 Berlandieri Torr.

86r9 canescens Muell. Arg.

8620 cardiophylla Muell. Arg.

862 I macrorhiza septemfida Engelm

8622 multifida L.

8623 spathulata sessiliflora

8624 stimulosa Michx.

Muell. Arg.

8625 Texana Muell. Arg.

\section{Manihot}





\section{Sebastiania}

8628 ligustrina Muell. Arg.

\section{Stillingia}

8629 angustifolia (Muell. Arg.)

Engelm. 8666 colorata Engelm.

8630 annua Muell. Arg.

863 I aquatica Chapm.

8632 dentata ('Torr.) Britton\&Rusby

8633 linearifolia Wats.

8634 paucidentata Wats.

8635 sylvatica L.

\section{Sapium}

8636 sebiferum Roxb.

(Stillingia sebiferum Michx.)

\section{Hippomane}

8637 Mancinella L.

\section{Gymnanthes}

8638 lucida Sw.

(Sebastiana lucida Muell. Arg.)

\section{Euphorbia}

8639 acuta Engelm.

8640 adenoptera Bertol.

864I albicaulis Rydb.

8642 albomarginata T. \& G.

8643 alta Norton

8644 ammannioides H. B. K.

8645 angusta Engelin.

8646 antisyphilitica Zucc.

8647 apocynifolia Small

8648 arenicola S. B. Parish

8649 Arizonica Engelm.

8650 Arkansana Engelm. \& Gray

865I atrosemina Norton

8652 Coloradensis Norton

8653 Missouriensis Norton

8654 barbellata Engelm.

8655 bicolor Engelm. \& Gray

8656 bifurcata Engelm.

8657 bilobata Engelm.

8658 Blodgetti Engelm.

8659 Brasiliensis Lam.

8660 brachycera Engelm.

866r buxifolia Lam.

(E. glabella of Chapm. Flora)
8662 campestris C. \& S.

8663 capitellata Engelm.

8664 chamaesula Boiss.

8665 subdentata (Engelm.)

Norton

8667 cordifolia Ell.

8668 commutata Engelm.

8669 erecta Norton

8670 corollata L.

867 I angustifolia Ell.

8672 glauca Millsp.

8673 Joorii Norton

8674 molle Millsp.

8675 crenulata Engelm.

8676 Franciscana Norton

8677 cuphosperma (Engelm.) Boiss.

8678 Curtissii Engelm.

8679 cuspidata Engelm.

8680 Cyparissias L.

868I Darlingtonii A. Gray

8682 deltoidea Michx.

8683 dentata Michx.

8684 gracillima Millsp.

8685 linearis Engelm.

8686 rigida Engelm.

8687 dictyosperma F. \& M.

8688 discoidalis Chapm.

8689 eriantha Benth.

8690 eriogonoides Small

869I Esula I.

8692 exigua L.

8693 exstipulata Engelm.

8694 Fendleri T. \& G.

8695 chaetocalyx Boiss.

8696 florida Engelm.

8697 Floridana Engelm.

(E. sphaerosperma Shuttlw.)

8698 Garberi Engelm.

8699 Geyeri Engelm. \& Gray

8700 glyptosperma Engelm.

8701 pubescens Boiss.

$87 \mathrm{O} 2$ tenerrima Engelm.

8703 Greenei Millsp.

8704 Helioscopia L.

8705 Helleri Millsp.

8706 heterophylla L. 

8708 hexagona Nutt.

8709 hirsuta ('Torr.) Wiegand

87 ro hirtula Engelm.

87 I I humistrata Engelm.

8712 inundata Torr.

8713 Ipecacuanhae L.

87I4 Jonesii Millsp.

8715 Laredana Millsp.

8716 lata Engelm.

87 I 7 Lathyris L.

87I 8 leiococca (Engelm.) Norton (E. Texana Boiss.)

87 I9 longicruris Scheele

8720 lucida W. \& K.

(E. Nicaeensis Wats. not All.)

872 I lurida Engelm.

8722 Pringlei (Engelm.) Norton

8723 maculata L.

8724 marginata Pursh

8725 Marylandica Greene

8726 mercurialina Michx.

8727 Mexicana (Engelm.) Norton (E. multicaulis Engelm.)

8728 misera Benth.

8729 mollis Engelm.

8730 montana Engelm.

8731 trifaris Norton

8732 Neo-Mexicana Greene

8733 nudicaulis Chapm.

8734 Nuttallii (Engelm.) Small (E. zygophylloides Boiss.)

8735 obtusata Pursh

8736 ocellata Dur. \& Hilg.

8737 occidentalis Drew

8738 odontadenia Boiss.

8739 olivacea Small

8740 Palmeri Engelm.

874 I peplifolia(Engelm.)Norton

8742 paniculata Ell.

8743 Parishii Greene

8744 Parryi Engelm.

8745 pediculifera Engelm.

8746 peplidion Engelm.

8747 Peplus L.

8748 pergamena Small

8749 petaloidea Engelm.

8750 pilulifera L.

875I discolor Engelm.
$875^{2}$ procumbens Boiss.

8753 platyphylla L.

8754 literata (Jacq.) Koch

8755 platysperma Engelm.

8756 Plummerae Wats.

8757 polycarpa Benth.

$875^{8}$ vestita Wats.

8759 polyclada Boiss.

8760 polygonifolia $\mathrm{L}$.

876I polyphylla Engelm.

8762 Preslii Guss.

(E. nutans of authors, not Lag.)

8763 prostrata Ait.

8764 pseudoserpyllifolia Millsp.

8765 pubentissima Michx.

8766 pycnanthema Engelm.

8767 radians Benth.

8768 Rattani Wats.

8769 revoluta Eingelm.

8770 robusta (Engelm.) Small

8771 interioris Norton

8772 Roemeriana Scheele

8773 rugulosa (Engelm.) Greene

8774 Rusbyi Greene

8775 schizoloba Engelm.

8776 mollis (Engelm.) Norton

8777 serpens H. B. K.

8778 flexicaulis (Scheele) Coult.

8779 serpyllifolia Pers.

8780 consanguinea Boiss.

878I serrula Engelm.

8782 setiloba Engelm.

8783 strictior Holzinger

8784 stictispora Engelm.

8785 Texensis Millsp.

8786 subpubens Engelm.

8787 telephioides Chapm.

8788 tetrapora Engelm.

8789 trachysperma Engelm.

8790 trichotoma Kunth

879 I versicolor Greene

8792 vilifera Scheele

8793 Wrightii T. \& G.

8794 zinniiflora Small

CALLITRICHACEAE

Callitriche

8795 Austini Engelm. 

8796 bifida (L.) Morong (C. autumnalis L.)

8797 Bolanderi Hegelm.

8798 deflexa A. Br.

8799 heterophylla Pursh

8800 longipedunculata Morong

88or marginata Torr.

8802 Nuttallii Torr.

8803 palustris $\mathrm{L}$.

8804 peploides Nutt.

8805 sepulta Wats.

8806 stenocarpa Hegelm.

EMPETRACEAE

\section{Corema}

8807 Conradii Tort.

\section{Empetrum}

8808 nigrum L.

\section{Ceratiola}

8809 ericoides Michx.

BUXACEAE

\section{Pachysandra}

88Io procumbens Michx.

Simondsia

88I I Californica Nutt.

\section{LIMNA NTHACEAE}

Limnanthes

88 I 2 alba Hartweg

88I3 Douglasii R. Br.

8814 floccosa Howell

88 I 5 gracilis Howell

8816 Macounii Trelease

88I 7 pumila Howell

8818 rosea Hartweg

\section{Floerkea}

8819 occidentalis Rydb.

8820 proserpinacoides Willd.

\section{ANACARDIACEAE}

\section{Schinus}

882 I molle L.

\section{Cotinus}

8822 cotinoides (Nutt.) Britton (Rhus cotinoides Nutt.)
Rhus.

8823 aromatica Ait.

(R. Canadensis Marsh.)

8824 mollis (A. Gray) Ashe

8825 Blodgettii Kearney

8826 Caroliniana Ashe

8827 copallina L.

8828 lanceolata A. Gray

8829 leucantha (Jacq.) DC.

8830 diversiloba T. \& G.

883I glabra L.

8832 hirta (L.) Sudw. (R. typhina L.)

8833 integrifolia (Nutt.) B. \& H.

8834 laurina Nutt.

8835 Metopium L.

8836 Mexicana A. Gray

8837 Michauxii Sargent

8838 microphylla Engelm.

8839 ovata Wats.

8840 radicans L.

884I Rydbergii Small

8842 Toxicodendron L.

8843 trilobata Nutt.

8844 quinata Jepson

8845 vernix L.

$(R$. venenata $\mathrm{DC}$.)

8846 virens Lindl.

\section{CYRILLACEAE}

\section{Cliftonia}

8847 monophylla (Lam.) Sargent (C. ligustrina Sims)

\section{Cyrilla}

8848 parviflora Shuttlw.

8849 racemiflora L.

\section{AQUIFOLIACEAE}

\section{Ilex}

8850 ambigua (Michx.) Chapm.

$885 \mathrm{I}^{\prime}$ coriacea 'Trelease

8852 Amelanchier M. A. Curtis

8853 Beadle Ashe

8854 Cassine L.

(I. Dahoon Walt.)

8855 angustifolia Willd.

8856 myrtifolia (Walt.) Sargent 

8857 decidua Walt.

$885^{8}$ glabra (L.) A. Gray

8859 laevigata (Pursh) A. Gray

8860 lanceolata Chapm.

886r longipes Chapm.

8862 lucida (Ait.) T. \& G.

8863 mollis A. Gray

8864 monticola A. Gray

8865 opaca Ait.

8866 verticillata (L.) A. Gray

8867 chrysocarpa Robinson

8868 cyclophylla Robinson

8869 padifolia (Willd.) T. \& G.

8870 tenuifolia (Torr.) Wats.

887 r vomitoria Ait.

(I. Cassine Walt.)

\section{Ilicioides}

8872 mucronata (L.) Britton

(Nemopanthes Canadensis DC.)

\section{CELASTRACEAE}

\section{Euonymus}

8873 Americanus L.

8874 angustifolius Wood

8875 sarmentosus Nutt.

8876 atropurpureus Jacq.

8877 Europaeus L.

8878 obovatus Nutt.

8879 occidentalis Nutt.

8880 Parishii Trelease

Celastrus

888I scandens L.

\section{Maytenus}

8882 plyllanthoides Benth.

\section{Pachystima}

8883 Canbyi A. Gray

8884 Myrsinites Raf.

\section{Gyminda}

8885 Grisebachii Sargent

(Myginda latifolia of Chapm.)

8886 glaucifolia (Griseb.) Sudw.

\section{Myginda}

8887 ilicifolia L.

8888 integrifolia Lam.
8889 Rhacoma Sw.

\section{Mortonia}

8890 Greggii A. Gray

889 I scabrella A. Gray

8892 sempervirens A. Gray

Forsellesia

(Glossopetalon)

8893 arida (Jones) Heller

8894 meionandra (Koehne) Heller

8895 Nevadensis (A. Gray) Heller

8896 pungens ('T. S. Brandegee)

Heller

8897 spinescens (A. Gray) Greene

\section{Schaefferia}

8898 cuneifolia A. Gray

8899 frutescens Jacq.

\section{Canotia}

8900 holacantha Torr.

\section{HIPPOCRATEACEAE}

\section{Hippocratea}

89oi ovata Lam.

\section{STAPHYLEACEAE}

Staphylea

8902 Bolanderi A. Gray

8903 trifolia L.

\section{ACERACEAE}

8904 barbatum Michx.

8905 Californicum (T. \& G.) Pax

(Negundo Californicum 'T. \& G.)

8906 circinatum Pursh

8907 Drummondii H. \& A.

8908 Floridanum (Chapm.) Pax

8909 glabrum Torr.

89 ro grandidentatum Nutt.

89II leucoderme Small

8912 macrophyllum Pursh

89r 3 Negundo L.

\section{(Negundo aceroides Moench)}

89I4 Texanum Pax

8915 nigrum Michx.

8916 Pennsylvanicum L. 

8917 rubrum I.

8918 Rugelii Pax

8919 saccharinum L. (A. dasycarpum Ehrh.)

8920 saccharum Marsh.

(A. saccharinum Wang.)

892 I spicatum Lam.

8922 tripartitum Nutt.

\section{HIPPOCASTANACEAE}

\section{Aesculus}

8923 arguta Buckley

8924 Californica (Spach) Nutt.

8925 glabra Willd.

8926. Hippocastanum L.

8927 octandra Marsh.

$$
\text { (A. flava Ait.) }
$$

8928 hybrida (DC.) Sargent

8929 parviflora Walt. .

8930 Pavia L.

\section{SAPINDACEAE}

\section{Serjania}

893I brachycarpa A. Gray

8932 incisa Torr.

8933 racemosa Shuttlw.

\section{Urvillea}

8934 ulmacea H. B. K.

\section{Cardiospermum}

8935 Halicacabum L.

8936 microcarpon H. B. K.

8937 molle L.

\section{Sapindus}

8938 Drummondii H. \& A.

8939 marginatus Willd.

8940 Saponaria L.

\section{Dodonaea}

894 I viscosa L.

8942 angustifolia (L. f.) Benth. 8943 spathulata (Sm.) Benth.

\section{Hypelate}

8944 trifoliata Sw.

\section{Exothea}

8945 paniculata (Juss.) Radlk.

\section{Ungnadia}

8946 speciosa Endl.

\section{BALSA MINACEAE}

\section{Impatiens}

8947 aurea Muhl.

$$
\text { (I. pallida Nutt.) }
$$

8948 biflora Walt.

(I. fulva Nutt.)

\section{RHA M NCEAE}

\section{Zizyphus}

8949 lycioides A. Gray

8950 canescens A. Gray

895I obtusifolius A. Gray

$895^{2}$ Parryi Torr.

\section{Condalia}

8953 Mexicana Schlecht.

8954 obovata Hook.

8955 spathulata A. Gray

\section{Microrhamnus}

8956 ericoides A. Gray

Reynosia

8957 latifolia Griseb.

\section{Rhamnidium}

$895^{8}$ ferreum (Vahl) Sargent (Condalia ferrea Griseb.)

\section{Karwinskia}

8959 Humboldtiana Zucc.

\section{Berchemia}

8960 scandens (Hill) Trelease (B. volubilis DC.)

\section{Sageretia}

896I Michauxii Brong.

8962 Wrightii Wats.

\section{Rhamnus}

8963 alnifolia L'Her.

8964 anonaefolia Greene

8965 betulaefolia Greene

8966 Californica Esch.

8967 Caroliniana Walt. (Hypelate paniculata Camb.) 8968 cathartica L. 

8969 crocea Nutt.

8970 pilosa Trelease

897 I Frangula L.

8972 ilicifolia Kellogg

8973 insularis Greene

8974 lanceolata Pursh

8975 occidentalis Howell

8976 pirifolia Greene

8977 Purshiana DC.

8978 rubra Greene

8979 Smithii Greene

8980 tomentella Greene

\section{Ceanothus}

898I Americanus I.

8982 intermedius (Pursh)

\section{Trelease}

8983 Andersoni Parry

8984 arboreus Greene

8985 Californicus Kellogg

8986 connivens Greene

8987 cordulatus Kellogg

8988 crassifolius Torr.

8989 cuneatus Nutt.

8990 ramulosus Greene

8991 decumbens Wats.

8992 dentatus T. \& G.

8993 divaricatus Nutt.

8994 divergens Parry

8995 diversifolius Kellogg

8996 eglandulosus ('Torr.) Trelease

8997 Fendleri A. Gray

8998 viridis Jones

8999 floribundus Hook.

9000 foliosus Parry

9001 glaber (Wats.) Trelease

9002 Greggii A. Gray

9003 hirsutus Nutt.

9004 impressus Trelease

9005 incanus T. \& G.

9006 integerrimus H. \& A.

9007 intricatus Parry

9008 Jepsoni Greene

9009 laevigatus ('T. \& G.) Howell

9010 Lemmoni Parry

90I I leucodermis Greene

9012 macrocarpus Nutt.

90I3 Martini Jones
9014 megacarpus Nutt.

90I5 microphyllus Michx.

9016 Orcuttii Parry

9017 ovatus Desv.

90I 8 pubescens 'T. \& G.

9019 Palmeri Trelease

9020 papillosus T. \& G.

902 I Parryi Trelease

9022 parvifolius (Wats.) Trelease

9023 perplexans Trelease

9024 pinetorum Coville

9025 prostratus Benth.

9026 pumilus Greene

9027 rigidus Nutt.

9028 grandifolius Torr.

9029 rugosus Greene

9030 sanguineus Pursh

903 I serpyllifolius Nutt

9032 sorediatus H. \& A.

9033 spinosus Nutt.

9034 thyrsiflorus Esch.

9035 griseus Trelease

9036 tomentosus Parry

9037 Veitchianus Dougl.

9038 velutinus Dongl.

9039 verrucosus Nutt.

9040 vestitus Greene

\section{Colubrina}

904I Colubrina (L.) Sargent

(C. ferruginosa Brong.)

9042 reclinata (L'Her.) Brong.

9043 Texensis (T. \& G.) A. Gray

\section{Adolphia}

9044 Californica Wats.

9045 infesta Meisner

\section{Gouania}

9046 Domingensis L.

\section{VITACEAE}

\section{Vitis}

9047 acida L.

9048 aestivalis Michx

9049 Bourquiniana (Munson)

Bailey

9050 glauca (Munson) Bailey

905I Linsecomii Munson 

9052 araneosa LeConte

9053 Arizonica Engelm.

9054 Berlandieri Planch.

9055 Baileyana Munson

9056 bicolor LeConte

9057 Californica Benth.

9058 candicans Engelm.

9059 coriacea (Shuttlw.) Bailey 9060 Caribaea DC.

906r Champini Planch.

9062 cinerea Eingelm.

9063 canescens (Engelm.) Bailey

9064 Floridana Munson

9065 cordifolia Michx.

9066 foetida Engelm.

9067 Helleri Bailey

9068 sempervirens Munson

9069 Doaniana Munson

9070 Girdiana Munson

907 I Labrusca L.

9072 Longii Prince

9073 microsperma (Munson)

9074 monticola Buckley

9075 Munsoniana Simpson

9076 palmata Vahl.

9077 rotundifolia Michx.

9078 rupestris Scheele

9079 dissecta Eggert

9080 Simpsoni Munson

908i Treleasei Munson

9082 vulpina $L$.

9083 praecox (Engelm.) Bailey

\section{Parthenocissus}

(Ampelopsis in part)

9084 heptaphylla (Buckley) Britton 9085 quinquefolia (L.) Planch. 9086 laciniata Planch.

\section{Ampelopsis}

9087 arborea (L.) Rusby

(Cissus stans Pers.)

9088 cordata Michx.

(Cissus Ampelopsis Pers.)

\section{Cissus}

9089 acida L. 9090 incisa Desmoul
909I sicyoides L.

9092 Floridana Planch.

\section{TILIACEA E}

\section{Corchorus}

9093 hirtus glabellus A. Gray 9094 siliquosus I.

\section{Tilia}

9095 Americana L.

9096 lieterophylla Vent.

9097 pubescens Ait.

9098 leptophylla Vent.

\section{Triumfetta}

9099 semitriloba L.

\section{IALVACEAE}

\section{Abutilon}

9roo Abutilon (L.) Rusby (A. Avicennae Gaertn.)

9ror Berlandieri A. Gray

9102 crispum Don

9103 imberbe Griseb.

9104 incanum (Link) Sweet

9105 Indicum hirtum (Lam.)Griseb.

9I06 Jacquinii Don

9107 Lemmoni Wats.

9I08 malacum Wats.

9109 Parishii Wats.

9I Io parvum A. Gray

9I II pedunculare H. B. K.

9II 2 permolle Sweet

9 II 3 reventum Wats.

9II4 Sonorae A. Gray

9II5 Thurberi A. Gray

9116 Wrightii A. Gray

\section{Wissadula}

9II7 holosericea (Scheele) Guerke 9II 8 rostrata Planch

\section{Horsfordia}

9II9 alata (Wats.) A. Gray

9I 20 Newberryi (Wats.) A. Gray

\section{Sphaeralcea}

9I 2 I ambigua A. Gray

9122 angustifolia Don

9123 violacea Davy 

9I24 asplenifolia Greene

9125 Coulteri (Wats.) A. Gray

9 26 cuspidata (A. Gray) Britton

9127 Emoryi Torr.

9128 Fendleri A. Gray

9129 fulva Greene

9I30 hastulata A. Gray

9I3I incana Torr.

91 32 dissecta A. Gray

9133 leptosepala Torr.

9r34 Lindheimeri A. Gray

9I35 lobata Wooton

91 36 perpallida Cockerell

9r37 longisepala Torr.

9I $3^{8}$ Munroana (Dougl.) Spach

9I39 Orcuttii Rose

9r40 Palmeri Rose

9I4I pedata Torr.

9142 angustiloba A. Gray

9143 pedatifida A. Gray

9144 rivularis Hook.) Torr.

(S. acerifolia Nutt.)

9r45 Rusbyi A. Gray

9I46 scabra Greene

9I47 subhastata Coult.

9I48 valida Greene

9I49 variabilis Cockerell

9I 50 Wrightii A. Gray

\section{Modiola}

9I 5 I Caroliniana (L.) Don

(M. multifida Moench)

\section{Lavatera}

9r52 assurgentiflora Kellogg

9r 53 insularis Wats.

\section{Althaea}

9r 54 cannabina L. 9I 55 officinalis L.

\section{Malva}

9I 56 Alcea L.

9 I 57 borealis Wallm.

9I 58 crispa L.

9I 59 moschata L.

9160 Nicaeensis Allioni

9I6I parviflora L.

9162 pusilla Sinith 9r63 rotundifolia $\mathrm{L}$.

9164 sylvestris $I$.

9165 verticillata $L$.

\section{Callirhoe}

9I66 alceoides (Michx.) A. Gray

9167 digitata Nutt.

9168 getanioides Small

9169 involucrata ( $T$. \& G.) A. Gray

9I 70 lineariloba A. Gray

9I7I Papaver (Cav.) A. Gray

9172 pedata A. Gray

9173 scabriuscula Robinson

9I74 triangulata (Leavenw.) A.Gray

\section{Sidalcea}

9I75 asplenifolia Greene

9I 76 asprella Greene

9I 77 Californica (Nutt.) A. Gray

9I 78 calycosa Jones

9179 campestris Greene

9I80 candida A. Gray

9r8I delphinifolia (Nutt.) Greene

9182 diploscypha ('T. \& G.) A. Gray

9r83 minor A. Gray

9I84 glaucescens Greene

9185 Hartwegi A. Gray

9I86 Hendersoni Wats.

9187 Hickmani Greene

9188 Parishii Robinson

9189 hirsuta A. Gray

9I90 humilis A. Gray

9I9I malvaeflora (Moc. \& Sesse)

A. Gray

9192 Neo-Mexicana A. Gray

9I93 nitrophila S. B. Parish

9I94 Oregana (Nutt.) A. Gray

9I95 parviflora Greene

9196 Thurberi Robinson

9197 pedata A. Gray

9198 reptans Greene

9199 scabra Greene

9200 secundiflora Greene

9201 spicata Greene

9202 sulcata Curran

9203 tenella Greene

9204 valida Greene

9205 virgata Howell 

Hesperalcea

9206 malachroides (H. \& A.) Greene

(Sidalcea malachroides A. Gray)

\section{Napaea}

9207 dioica L.

\section{Malvastrum}

9208 aboriginum Robinson

9209 Americanum (L.) Tors.

(M. tricuspidatum A. Gray)

92 Io angustum A. Gray

92 I I arcuatum (Greene Robinson

92 I 2 coccineum (Pursh) A. Gray

92 I 3 Davidsonii Robinson

92 I 4 densiflorum Wats.

92 I 5 dissectum (Nutt.) Cockerell

9216 exile A. Gray

9217 fasciculatum (Nutt.) A. Gray

9218 Fremontii Torr.

9219 cercophorum Robinson

9220 involucratum Robinson

922 I leptophyllum A. Gray

9222 marrubioides Dur. \& Hilg.

9223 paniculatum A. Gray

9224 multicaule (Scheele) Britton

9225 multiflorum Greene

9226 nesioticum Robinson

9227 orbiculatum Greene

9228 Palmeri Wats.

9229 Parryi Greene

9230 rotundifolium A. Gray

923I Rugelii Wats.

9232 scoparium (L'Her) A. Gray

9233 spicatum (L.) A. Gray

9234 splendidum Kellogg

9235 Thurberi A. Gray

9236 Wrightii A. Gray

\section{Sida}

9237 acuta Burm.

9238 angustifolia Lam.

9239 Canariensis Willd.

9240 carpinifolia L. f.

924 I ciliaris $\mathrm{L}$.

6242 cordifolia L.

9243 cuneifolia A. Gray

9244 diffusa H. B. K.

9245 Elliottii T. \& G.

9246 parviflora Chapm.
9247 fasciculata T. \& G.

9248 filipes A. Gray

hastata St. Hil.

(S. physocalyx A. Gray)

9250 hederacea (Dougl.) Torr.

925I Helleri Rose

9252 hermaphrodita (L.) Rusby

(S. Napaea Cav.)

9253 lepidota A. Gray

9254 sagittaefolia A. Gray

9255 leptophylla Small

9256 Lindheimeri Engelm. \& Gray

9257 longipes A. Gray

$925^{8}$ Neo-Mexicana A. Gray

9259 rhombifolia E.

9260 rubro-marginata Nash

926I spinosa L.

9262 supina L'Her.

9263 tragiaefolia A. Gray.

\section{Anoda}

9264 abutiloides A. Gray

9265 hastata Cav.

9266 lavateroides Medic.

9267 pentaschista A. Gray

9268 obtusior Robinson

9269 reticulata Wats.

9270 Thurberi A. Gray

927 I triangularis (Willd.) DC.

9272 digitata (A.Gray) Robinson

9273 Wrightii A. Gray

\section{Malachra}

9274 alcaefolia rotundifolia (Sch.)

9275 capitata I. Guerke

\section{Urena}

9276 lobata L.

\section{Pavonia}

9277 hastata Cav.

9278 lasiopetala Scheele

9279 racemosa Sw.

9280 spinifex (L.) Cav.

\section{Malvaviseus}

928I Drummondii T. \& G.

\section{Hibiscus}

9282 aculeatus Walt.

9283 cardiophyllus A. Gray 

9284 cocineus Walt.

9285 Coulteri Harv.

9286 denudatus Benth.

9287 involucellatus A. Gray

9288 esculentus L.

9289 furcellatus Lam.

9290 grandiflorus Michx.

9291 incanus Wendl.

9292 integrifolius (Chapm.) Small

9293 lasiocarpus Cav.

9294 occidentalis ('Torr.) A.Gray

9295 Manihot L.

9296 militaris Cav.

9297 Moscheutos L.

9298 Syriacus L.

9299 Trionum L.

9300 tubiflorus DC.

\section{Paritium}

930r tiliaceum (L.) A. Juss.

(Hibiscus tiliaceus L.)

\section{Thespesia}

9302 populnea (L.) Soland.

\section{Kosteletzkya}

9303 altheaefolia (Chapm.) Rusby.

9304 amilacifolia (Chapm.) A. Gray

9305 Virginica (L.) A. Gray

\section{Cienfugosia}

(Fugosia)

9306 heterophylla (Spach) Guerke 9307 sulphurea (St. Hil.) Garcke

(Fugosia Drummondii A. Gray)

\section{Gossypium}

9308 Barbadense L.

9309 herbaceum L.

\section{Ingenhouzia}

9310 triloba DC.

\section{STERCULIACEAE}

\section{Fremontodendron}

(Fremontia)

93 I Californicum (Torr.) Coville

\section{Hermannia}

93I2 pauciflora Wats.

93I3 Texana A. Gray

\section{Melochia}

9314 corchorifolia L.

93I5 hirsuta Cav.

93I6 glabrescens (Presl) A. Gray

9317 pyramidata L.

93I 8 tomientosa $\mathrm{L}$.

\section{Nephropetalum}

93I9 Pringlei Robinson \& Greenm.

\section{Waltheria}

9320 Americana L.

932 I detonsa A. Gray

\section{Ayenia}

9322 microphylla A. Gray

9323 pusilla L.

\section{DILLENIACEAE}

\section{Crossosoma}

9324 Bigelovii Wats.

9325 Californica Nutt.

\section{THEACEAE}

\section{Gordonia}

9326 Altamaha (Marsh.) Sargent (G. pubescens L'Her.)

9327 Lasianthus (L.) Ellis

\section{Stuartia}

9328 Malachodendron L. (S. Virginica Cav.)

9329 pentagyna L'Her.

\section{GUTTIFERACEAE}

\section{Clusia}

9330 flava L.

\section{HYPERICACEAE}

\section{Ascyrum}

933I amplexicaule Michx.

9332 hypericoides L.

$$
\text { (A. Crux-Andreae L.) }
$$

9333 multicaule Michx.

9334 pumilum Michx.

9335 stans Michx.

9336 tetrapterum (Lam.) Vahl 



\section{Hypericum}

9337 acutifolium Ell.

$933^{8}$ adpressum Bart.

9339 anagalloides C. \& S.

9340 Nevadense Greene

934I apocynifolium Small

9342 Ascyron L.

9343 aspalathoides Willd.

9344 aureum Bart.

9345 Buckleii M. A. Curtis

9346 boreale (Britton) Bicknell

9347 Canadense L.

9348 cistifolium Lam.

9349 concinnum Benth.

9350 densiflorum Pursh

935I dolabriforme Vent.

9352 Drummondii (Grev. \& Hook.)

9353 ellipticum Hook.

9354 fasciculatum Lam.

9355 formosum H. B. K.

$935^{6}$ galioides Lam.

9357 glomeratum Small

$935^{8}$ graveolens Buckley

9359 gymnanthum Engelm. \& Gray

9360 Kalmianum I.

936r lobocarpum Gattinger

9362 maculatum Walt.

9363 majus (A. Gray) Britton

9364 microsepalum A. Gray

9365 mutilum L.

9366 myrtifolium Lam.

9367 nudiflorum Michx.

9368 opacum T. \& G.

9369 perforatum L.

9370 pilosum Walt.

937 I prolificum L.

9372 Scouleri Hook.

9373 sphaerocarpum Michx.

9374 virgatum Lam.

9375 ovalifolium Britton

\section{Sarothra}

9376 gentianoides L.

(Hypericum nudicaule Walt.) 9403 ericoides L.

\section{Triadenum}

\section{(Elodea)}

9377 longifolia Small

9378 petiolatum (Walt.) Britton
9379 Virginicum (L.) Raf. (Elodea campanulata Pursi)

\section{ELATINACEAE}

\section{Bergia}

9380 Texana (Hook.) Suith

\section{Elatine}

938I Americana (Pursh) Arn.

9382 brachysperma A. Gray

$93^{8} 3$ Californica A. Gray

9384 rubella Rydb.

9385 triandra Schk.

9386 Williamsii Rydb.

\section{FRANKENIACEAE}

\section{Frankenia}

9387 grandifolia C. \& S.

9388 campestris A. Gray

9389 Jamesii Torr.

9390 Palmeri Wats.

\section{TAMARICACEAE}

\section{Tamarix}

939I Gallica L.

\section{Fouquiera}

9392 splendens Fingelm.

\section{CIST A CEA E}

\section{Helianthemum}

9393 Aldersonii Greene

9394 arenicola Chapm.

9395 Canadense (L.) Michx.

9396 capitatum Nutt.

9397 Carolinianum Michx.

9398 corymbosum Michx.

9399 Greenei Robinson

9400 majus (L.) B. S. P.

940I Nashii Britton

9402 scoparium Nutt.

\section{Hudsonia}

9404 montana Nutt.

9405 tomentosa Nutt.

\section{Lechea}

9406 divaricata Shuttlw. 

9407 Drummondii (Spach) T. \& G. 9432 Beckwithii T. \& G.

9408 intermedia Leggett

9409 juniperma Bicknell

9410 Leggettii Britton \& Hollick

94 I I maritima Leggett

94 I 2 minor L.

$$
\text { (L. thymifolia Michx.) }
$$

94I 3 patula Leggett

94I 4 racemulosa Michx.

94I 5 stricta Leggett

94I6 tenuifolia Michx.

9417 Torreyi Leggett

$94 \mathrm{I} 8$ villosa Eill.

(L. major Michx.

\section{BIXACEAE}

\section{Amoreuxia}

94I9 palmatifida DC.

9420 Wrightii A. Gray

WINTERANACEAE

Canella

942 I Winterana (L.) Gaertn.

KOEBERLINACEAE

Koeberlinia

9422 spinosa Zucc.

\section{VIOLACEAE}

\section{Calceolaria}

(Ionidium)

9423 riparia (H. B. K.) Kuntze

9424 verticillata (Ortega) Kuntze

(I. polygalaefolium Vent.)

\section{Cubelium}

(Solea)

9425 concolor (Forst.) Raf.

\section{Viola}

9426 adunca Smith

8427 affinis LeConte

( $V$. obliqua Hill?)

9428 amorphophylla Pollard

9429 arenaria DC.

( $V$. canina var. puberula Wats.) 9430 atriplicifolia Greene 943I aurea Kellogg
9433 Bernardi Greene

9434 biflora L.

9435 blanda I.

9436 Brittoniana Pollard (V. Atlantica Britton)

9437 Brooksii Kellogg

( $V$. lobata var. integrifolia Wats.)

$943^{8}$ Canadensis L.

9439 scariosa Porter

9440 scopulorum A. Gray

944 I cognata Greene

9442 conjugens Greene

9443 cucullata Ait.

9444 cuneata Wats.

9445 cuspidata Greene

9446 cyclophylla Greene

9447 dentata Pursh

(V. Porteriana Pollard)

9448 Dicksonii Greene

9449 domestica Bicknell

$945^{\circ}$ Douglasii Steud.

945I elegantula Greene

9452 emarginata (Nutt.) LeConte

9453 erectifolia Aven Nelson

9454 esculenta E11.

9455 falcata Greene

9456 fimbriatula Smith

(V. ovata Nutt.)

9457 flavovirens Pollard

6458 Flettii Piper

9459 glabella Nutt.

9460 Hallii A. Gray

946I hastata Michx.

9462 Howellii A. Gray

9463 Labradorica Schrank

(canina var. Muhlenbergii)

9464 lanceolata L.

9465 Langloisii Greene

9466 Langsdorfii Fisch.

9467 Leconteana Don

(blanda var. palustriformis A. Gray)

9468 linguaefolia Nutt.

9469 lobata Benth.

9470 longipes Nutt.

947 I Macounii Greene

9472 Missouriensis Greene

9473 Mistassinica Greene 

9474 Montanensis Rydb.

9475 monticola Rydb.

9476 multicaulis ('T. \& G.) Britton

9477 nephrophylla Greene

9478 Nuttallii Pursh

9479 ocellata Pursh

9480 odontophora Rydb.

948 I odorata L.

9482 orbiculata Geyer

9483 oxyceras (Wats.) Greene

9484 palmata L.

9485 palmata dilatata Ell.

9486 palustris L.

9487 papilionacea Pursh

(communis Pollard)

9488 pedata L.

9489 bicolor Pursh

9490 pedatifida Don

949I pinetorum Greene

9492 praemorsa Dougl.

9493 pratincola Greene

9494 populifolia Greene

9495 primulaefolia L.

9496 occidentalis A. Gray

9497 pruinosa Pollard

9498 pubescens Ait.

9499 purpurea Kellogg

9500 Rafinesquii Greene (tricolor var. arvensis DC.)

950r renifolia A. Gray

9502 retusa Greene

9503 rostrata Pursh

9504 rotundifolia Michx.

9505 sagittata Ait.

9506 scabriuscula ('T.\&G.) Schwein.

9507 Selkirkii Pursh

9508 sempervirens Greene

(sarmentosa Dougl.)

9509 septemloba LeConte

9510 septentrionalis Greene

95I I Sheltoni Torr.

9512 sororia Willd.

95I3 striata Ait.

95I4 subsagittata Greene

95'5 subsinuata Greene

95 I 6 tricolor L.

9517 trinervata Howell

9518 tripartita Ell.
9519 glaberrima (Ging.) Harper

9520 vagula Greene

9521 vallicola Aven Nelson

9522 venosa (Wats.) Rydb.

$95^{2} 3$ venustula Greene

9524. vicinalis Greene

(insignis Pollard)

9525 villosa Walt.

9526 vittata Greene

9527 Watsonii Greene

TURNERACEAE

Piriqueta

9528 fulva Chapm.

\section{Turnera}

9529 diffusa aphrodisca Urban

\section{PASSIFLORACEAE}

\section{Passiflora}

9530 affinis Engelm.

953 I Bigelovii Small

9532 foetida $\mathrm{L}$.

9533 inamoena A. Gray

9534 incarnata L.

9535 lutea L.

9536 Mexicana Juss.

9537 multiflora I.

9538 suberosa L.

9539 angustifolia Masters

9540 pallida Masters

954I tenuiloba Engelm.

CARICACEAE

\section{Carica}

9542 Papaya L.

LOASACEAE

\section{Cevallia}

9543 sinuata Iag.

\section{Petalonyx}

9544 nitidus Wats.

9545 Thurberi A. Gray

\section{Mentzelia}

9546 affinis Greene

9547 albicaulis (Hook.) Dougl.

9548 aspera L. 

9549 Brandegei Wats.

9550 chrysantha Engelm.

955I compacta Aven Nelson

9552 congesta $T$. \& G.

9553 decapetala (Pursh) Urb. \& Gil. (M. ornata T. \& G.)

9554 densa Greene

9555 Floridana Nutt.

9556 gracilenta T. \& G.

9557 hispida Willd.

$955^{8}$ integrifolia (Wats.) Rydb. (M. dispersa Wats.)

9559 involucrata Wats.

9560 laevicaulis (Doug1.) T. \& G.

956r leucophylla T. S. Brandegee

9562 Lindleyi T. \& G.

9563 lutea Greene

9564 micrantha T. \& G.

9565 multiflora (Nutt.) A. Gray

9566 integra Jones

9567 Nelsoni Greene

9568 nitens Greene

9569 nuda (Pursh) T. \& G.

9570 oligosperma Nutt.

957 I parviflora Heller

9572 perennis Wooton

9573 pumila T. \& G.

9574 reflexa Coville

9575 Rusbyi Wooton

9576 tenerrima Rydb.

9577 Tweedyi Rydb.

9578 Torreyi A. Gray

9579 tricuspis A. Gray

9580 Wrightii A. Gray

\section{Eucnide}

958I bartonioides Zucc.

9582 lobata A. Gray

$95^{8} 3$ urens Parry

\section{Sympetaleia}

9584 aurea A. Gray

\section{DATISCACEAE}

Datisca

9585 glomerata (Presl) B. \& H.

\section{CACTACEAE}

\section{Cereus}

9586 aggregatus (Engelm.) Coult.
$95^{87}$ Berlandieri Engelm.

9588 chloranthus Engelm.

9589 compressus Mill.

9590 ctenoides Engelm.

959I dasyacanthus Engelm.

9592 Neo-Mexicanus Coult.

9593 dubius Engelm.

9394 Emoryi Engelm.

9595 Engelmanni Parry

9596 chrysocentrus Engelm.

9597 variegatus Engelm.

9598 enneacanthus Engelm.

9599 Fendleri Engelm.

9600 giganteus Engelm.

960I gonacanthus Eingelm.

9602 Greggi Engelm.

9603 hexandrus Engelm.

9604 Mohavensis Engelm.

9605 Zuniensis Engelm.

9606 monoclonus DC.

9607 octacanthus (Muhlenpf.)Coult.

9608 paucispinus Engelm.

9609 pectinatus centralis Coult.

9610 rigidissimus Engelm.

96I I spinosus Coult.

9612 polyacanthus Engelm.

96r 3 Poselgeri Coult.

96I4 Roetteri Engelm.

96r 5 Roemeri Muhlenpf.

96r6 Schottii Engelm.

96I7 stramineus Engelm.

9618 Thurberi Engelm.

9619 triglochidiatus Engelm.

\section{Echimocereus}

9620 caespitosus Engelm.

962 I castaneus (Engelm.) Heller

9622 viridiflorus Engelm.

9623 tubulosus (Coult.) Heller

\section{Echinocactus}

9624 bicolor Schottii Engelm.

9625 brevihamatus Engelm.

9626 cylindraceus Engelm.

9627 Emoryi Engelmi.

9628 erectocentrus Coult.

9629 hamatocanthus Muhlenpf.

9630 brevispinus(Engelm.)Coult

963 I longihamatus (Gal.) Coult. 

9632 horizonthalonis Lemaire

9633 intertextus Engelm.

9634 dasyacanthus Engelm.

9635 Johnsoni Parry

9636 octocentrus Coult.

9637 Lecontei Engelm.

9638 limatus Engelm.

9639 papyracanthus Engelm.

9640 Parryi Engelm.

9641 polyancistrus Engelm. \& Big. 9684

9642 polycephalus Engelm. \& Big. 9685

9643 xeranthemoides Coult.

9644 pubispinus Engelm.

9645 Scheerii Salm.

9646 setispinus Engelm.

9647 Muhlenpfordtii(Fen.)Coult 9648 Sileri Engelm.

9649 Simpsoni Engelm.

9650 minor Engelm.

965I robustior Coult.

9652 sinuatus Dietr.

9653 Texensis Hoepf.

9654 uncinatus Gal.

9655 Wrightii Engelm.

9656 viridescens Nutt.

9657 Whipplei Engelm. \& Big.

9658 spinosior Eingelm.

9659 Wislizeni Engelm.

9660 albispinus 'Toumey

\section{Cactus}

\section{(Mammillaria)}

966I bispinus Coult.

9662 Brownii Toumey

9663 dasyacanthus(Fngelm.)K untze 9664 echinus (Engelm.) Kuntze 9665 Goodrichii (Scheer) Kuntze 9666 Grahami (Engelm.) Kuntze 9667 Heyderi (Muhlenpf.) Kuntze 9668 hemisphaerica (Fingelm.)

Coult.

9669 lasicanthus (Engelm.) Kuntze 9670 denudatus (Engelm.)CouIt. 967 I macromeris (Engelm.) Kuntze 9672 melacanthus (Engelm.)Kuntze 9673 micromeris (Engelm.) Kuntze 9674 Greggii (Engelm.) Coult. 9675 Missouriensis (Sweet) Kuntze
9676

9677

9678

9679

9680 Potsii (Scheer) Kuntze

968I radians (DC.) Knntze

9682 radiosus (Engelm.) Conlt.

9683 Alversoni Coult.

Arizonicus(Engelm.)Coult. chloranthus (Engelm.)

Coult.

9686

9687

deserti (Engelm.) Coult. Meo-Mexicanus (Engelm.)

Coult.

9688 Scheerii (Muhlenpf.) Kuntze 9689 scolymoides (Scheidw.)Kuntze 9690 sulcatus (Engelm.) Coult. 969 I sphaericus (Dietr.) Kuntze 9692 stellatus Texanus (Engelm.)

Coult.

9693 tetrancistrus (Eugelm.) Coult. 6694 tuberculosus (Engelm.)Kuntze 9695 viviparus Nutt.

9696 Wrightii (Engelm.) Kuntze

\section{Anhalonium}

9697 Engelmanni Lehm.

\section{Lophophora}

9698 L,ewinii (Henn.) Thompson 9699 Williamsii (Lemaire) Coult.

\section{Opuntia}

9700 acanthocarpa Engelm.

970 r angustata Engelm.

9702 arborescens Engelm.

9703 arbuscula Engelm.

9704 arenaria Engelm.

9705 basilaris Engelm.

9706 ramosa S. B. Parish

9707 Bernardina Engelm.

9708 Bigelovii Engelm.

9709 bulbispina Fingelm.

97ro Camanchica Engelm. \& Big.

97 I I chlorotica Engelm.

97 I 2 clavata Engelm.

97I3 Davisii Engelm.

97 I 4 echinocarpa Engelm. 



9715 Parkeri (Engelm.) Coult.
9716 robustior Coult.
97 I7 Emoryi Engelm.
9718 Ficus-Indica Mill.
97 19 filipendula Engelm.
9720 fragilis (Nutt.) Haw.
9721 brachyarthra Engelm.
9722 fulgida Engelm.

9723 mamillata (Schott) Coult. 9724 fusco-atra Engelm.

9725 Grahami Engelm.

9726 Greenei (Coult.) Engelm.

9727 humifusa Raf.
(O. mesacantha Raf.)

9728 cymochila(Engelm.)Heller

9729 grandiflora(Engelm.)Heller

9730 microsperma (Engelm.)

793I parva (Coult.) Heller Heller

9732 stenochila (Engelm.)Heller

9733 Vaseyi (Coult.) Heller

9734 hystricina Engelm.

9735 Kleiniae DC.

9736 laevis Coult.

9737 leptocaulis DC.

$973^{8}$ stipata Coult.

9739 vaginata (Engelm.) Wats.

9740 Lindheimeri Engelm.

974I cyclodes (Engelm.) Coult.

9742 dulcis (Engelm.) Conlt.

9743 littoralis (Engelm.) Coult.

9744 occidentalis (Engelm)Coult

9745 macrocentra Engelm.

9746 macrothiza Engelm.

9747 Mohavensis Engelm. \& Big.

9748 oplocarpa (Conlt.) Engelm.

9749 Opuntia (L.) Coult.

(O. vulgaris Mill.)

9750 Palmeri Engelm.

975I Parryi Engelm.

9752 Pes-Corvi LeConte

9753 phaeacantha Engelm.

9754 major Engelm.

9755 polyacantha Haw.

(O. Missouriensis DC.)

9756

9757

$975^{8}$
9759

9760

976 I trichophora (Engelm.)

9762 prolifera Engelm.

9763 pulchella Engelm.

9764 ramosissima Engelm.

9765 rubrifolia Engelm.

9766 rutila Nutt.

9767 Schottii Engelm.

9768 serpentaria Engelm.

9769 sphaerocarpa Eingelm.

9770 Utahensis Engelm.

977 I spinosior (Engelm.) Touney

9772 Neo-Mexicana Toumey

9773 Strigil Engelm.

9774 tenuispina Engelm.

9775 tortispina Engelm.

9776 Tuna (L.) Mill.

9777 Treleasii Coult.

9778 versicolor Engelm.

9779 Whipplei Engelm.

\section{THYMELACEAE}

\section{Daphne}

9780 Mezerum L.

\section{Dirca}

978I occidentalis A. Gray

9782 palustris I.

\section{ELAEAGNACEAE}

Lepargyraea

(Shepherdia)

9783 argentea (Nutt.) Greene

9784 Canadensis (L.) Greene

9785 rotundifolia (Parry) Greene

\section{Elaeagnus}

9786 argentea Pursh

9787 hortensis Bieb.

\section{LYTHRACEAE}

\section{Rotala}

9788 ramosior (L.) Koehne

\section{Ammannia}

9789 auriculata Willd.
9790 coccinea Rottb.
albispina (Engelm. \& Big.)
Coult. 979I Koehnei Britton platycarpa(Engelm.)Coult. 9792 latifolia L. 



\section{Didiplis}

9793 diandra (Nutt.) Wood (D. linearis Raf.)

\section{Lythrum}

9794 adsurgens Greene 9795 alatum Pursh

9796 pumilum A. Gray

9797 album H. B. K.

9798 Californicum T. \& G.

9799 flagellare Shuttlw.

9800 hyssopifolia L.

980I lanceolatum Ell.

9802 lineare $\mathrm{L}$.

9803 ovalifolium Engelm.

9804 Salicaria L.

9805 Sanfordi Greene

9806 Vulneraria Ait.

\section{Parsonsia}

\section{(Cuphea)}

9807 Chapinani Heller

(Cuphea aspera Chapm.)

9808 glutinosa (C. \& S.) Heller

9809 petiolata (L.) Rusby

(Cuphea viscosissima Jacq.)

981o procumbens (Cav.) Heller

98I I Wrightii (A. Gray) Kearney

Nesaea

9812 longipes A. Gray

\section{Heimia}

98I 3 salicifolia H. B. K.

\section{Decodon}

9814 verticillatus (L.) Eill.

\section{Lagerstroemia}

98 I 5 Indica L.

PUNICACEAE

\section{Punica}

98I6 granatum L.

\section{RHIZOPHORACEAE}

\section{Rhizophora}

98I7 Mangle L.

\section{MYRTACEAE}

\section{Psidium}

9818 buxifolium Nutt.

9819 Guajava L.

\section{Chytraculia}

(Calyptranthes)

9820 Chytraculia (L.) Sudw.

982I genuina (Berg.) Sudw.

9822 ovalis (Berg.) Sudw.

9823 trichotoma (Berg.) Sudw.

9824 pauciflora (Berg.) Kuntze

9825 Suzygium (L.) Kuntze

\section{Eugenia}

9826 buxifolia (Sw.) Willd.

9827 Cabanisiana Berg.

9828 dicrana Berg.

9829 Garberi Sargent

9830 longipes Berg.

983 I monticola (Sw.) DC.

9832 procera (Sw.) Poir.

\section{Anamomis}

9833 dichotoma (DC.) Sargent (Eugenia dichotoma DC.)

\section{COMBRETACEAE}

\section{Terminalia}

9834 Buceras (Browne) B. \& H.

9835 Cattapa L.

\section{Conocarpus}

9836 erecta L.

9837 arborea DC.

9838 procumbens (L.) DC.

9839 sericea DC.

\section{Laguncularia}

9840 racemosa (L.) Gaertn. f.

\section{MELASTOMACEAE}

\section{Rhexia}

984I aristosa Britton

9842 ciliosa Michx.

9843 glabella Michx.

9844 filiformis Small

9845 Floridana Nash

9846 lanceolata Walt. 

9847 lutea Walt.

9848 Mariana L.

9849 serrulata Nutt.

9850 stricta Pursh

985 I Virginica L.

\section{ONAGRACEAE}

\section{Jussiaea}

9852 decurrens (Walt.) DC.

9853 diffusa Forsk.

9854 erecta L.

9855 octonervia Lam.

9856 Peruviana L.

9857 pilosa H. B. K.

9858 repens $L$.

9859 Californica Wats.

9860 grandiflora Micheli

986 I suffruticosa L.

\section{Ludwigia}

9862 alata L.

9863 alternifolia L.

9864 linearifolia Britton

9865 capitata Michx.

9866 Curtisi Chapm.

9867 glandulosa Walt.

9868 hirtella Raf.

9869 lanceolata Ell.

9870 linearis Walt.

987 I puberula Engelm. \& Gray

9872 linifolia Poir.

9873 microcarpa Michx.

9874 pilosa Walt.

9875 polycarpa Short \& Peter

9876 sphaerocarpa Ell.

9877 virgata Michx.

\section{Isnardia}

(Ludwigia in part)

9878 natans (E11.) Small

9879 palustris L.

9880 spathulata ('T. \& G.) Small

\section{Ludwigiantha}

988I arcuata (Walt.) Small (Ludwigia arcuata Walt.)

\section{Zauschneria}

9882 Californica Presl

9883 microphylla A. Gray
9884 cana Greene

9885 latifolia Greene

9886 tomentella Greene

9887 villosa Greene

\section{Epilobium}

9888 adenocaulon Haussk.

9889 perplexans Trelease

9890 alpinum L.

989I anagallidifolium Lam.

9892 Behringianum Haussk.

9893 Bongardi Haussk.

9894 boreale Haussk.

9895 brevistylum Barbey

9896 Californicum Haussk.

9897 clavatum Trelease

9898 coloratum Muhl.

9899 albiflorum Cockerell

9900 Davuricum Fisch.

990I delicatum Trelease

9902 tenue Trelease

9903 Drummondii Haussk.

9904 latiusculum Rydb.

9905 exaltatum Drew

9906 Fendleri Haussk.

9907 Franciscanum Barbey

9908 glaberrimum Barbey

9909 latifolium Barbey

9910 glandulosum Lehm.

99I I Halleanum Haussk.

9912 Hammondii Howell

99I3 hirsutum L.

99I4 holosericeum Trelease

99I5 Hornemanii Reicherb.

9916 jucundum A. Gray

99I7 lactiflorum Haussk.

9918 leptocarpum Haussk.

9919 Macounii Trelease

9920 lineare Muhl.

9921 oliganthum Trelease

9922 luteum Pursh

9923 minutum Lindl.

9924 Bioletti Greene

9925 foliosum T. \& G.

9926 niveum Brandegee

9927 Novo-Mexicanum Haussk.

9928 obcordatum A. Gray

9929 occidentale (Trelease) Rydb. 

9930 Oregonense Hanssk.

993I gracillimum Trelease

9932 palustre L.

9933 paniculatum Nutt.

9934 Parishii Trelease

9935 rigidum Haussk.

9936 canescens Trelease

9937 saximontanum Haussk.

9938 strictum Muhl.

9939 subcaesium Greene

9940 suffruticosum Nutt.

994I ursinum Parish

9942 subfalcatum Trelease

9943 Watsoni Barby

\section{Chamaenirion}

(Epilobium in part)

9944 angustifolium (L.) Scop. (E. spicatum Lam.)

9945 latifolium (L.) Sweet

\section{Boisduvalia}

9946 bipartita Greene

9947 cleistogoma Curran

9948 densiflora (Lindl.) Wats. (B. Douglasii Spach)

9949 imbricata Greene

9950 diffusa Greene

995 I glabella (Nutt.) Walp.

9952 parviflora Heller

9953 stricta (A. Gray) Greene (B. Torreyi Wats.)

\section{Clarkia}

9954 elegans Lindl.

9955 pulchella Pursh

9956 rhomboidea Dougl.

9957 Saxeana Greene

$995^{8}$ virgata Greene

\section{Eucharidium}

9959 Breweri A. Gray

9960 concinnum F. \& M.

\section{Godetia}

996 I albescens Lindl.

9962 amoena Lilj.

9963 biloba (Durand) Wats.

9964 Bottae Spach

9965 epilobioides (Nutt.) Wats.
9966 grandiflora Lindl:

9967 hispidula Wats.

9968 lepida Lindl.

9969 Arnottii Wats.

9970 parviflora Wats.

997 I micropetala Greene

9972 pulcherrima Greene

9973 purpurea (Curtis) Wats.

9974 quadrivulnera Spach

9975 Romanzovii Spach

9976 tenella (Cav.) Wats.

9977 viminea Spach

9978 intermedia Kellogg

9979 Williamsoni (Dur. \& Hilg.)

\section{Onagra}

Wats.

(Oenothera in part)

9980 arguta (Greene) Small

998I biennis (L.) Scop.

9982 grandiflora (Ait.) Lindl.

9983 cruciata (Nutt.) Small

9984 depressa (Greene) Small

9985 Hookeri (T. \& G.) Small

9986 Jamesii (T. \& G.) Small

9987 macrosceles (A. Gray) Small 9988 Oakesiana (A. Gray) Britton

\section{Oenothera}

9989 Drummondii Hook.

9990 heterophylla Spach

999I humifusa Nutt.

9992 laciniata Hill

(O. simuata L.)

9993 grandis Britton

9994 occidentalis Small

9995 Mexicana Spach

9996 nyctaginiifolia Small

9997 rhombipetala Nutt.

\section{Anogra}

(Oenothera in part)

9998 albicaulis (Pursh) Britton

9999 Buffumi Aven Nelson

I0000 Californica (Wats.) Small

I000I coronopifolia (T.\&G.) Britton

I0002 deltoidea(Torr.\&Frem.)Small

I0003 Neo-Mexicana Small

I0004 pallida (Lindl.) Britton 

I0005 brevifolia(Engelm.)Small I0037 Missouriensis (Sims) Spach I0006 Engelmanni Small I0007 latifolia (Rydb.) Small I0008 runcinata(Engelm.)Small I0009 rhizomata Aven Nelson rooro simplex Small Ioor I trichocalyx (Nutt.) Small IOOI 2 xylocarpa (Coville) Small

\section{Kneiffia}

(Oenothera in part)

roor3 Alleni (Britton) Small Ioor4 fruticosa (L.) Raimann roor 5 glauca (Michx.) Spach Ioor6 linearis (Michx.) Spach roor 7 linifolia (Nutt.) Spach roor 8 longipedicellata Small roorg pilosella (Raf.) Heller I0020 pumila (L.) Spach roo2 I Spachiana ('T. \& G.) Small I0022 subglobosa Small

\section{Hartmannia}

(Oenothera in part) 10023 rosea (Ait.) Don IOO24 parvifolia (Coult.) Small I0025 speciosa (Nutt.) Small I0026 tetraptera (Cav.) Small

\section{Pachylophus}

(Oenothera in part)

I0027 caespitosus (Nutt.) Raimann I0028 montanus(Nutt.)A ven Nelson

\section{Gaurella}

(Oenothera in part) roo29 guttulata (Geyer) Small

\section{Lavauxia}

(Oenothera in part)

\section{Taraxia}

Io038 breviflora (T. \& G.) Nutt. I0039 gracilliflora (H. \& A.) Raim. I0040 longiflora Nutt.

I004I ovata (Nutt.) Small I0042 Palmeri (Wats.) Small 10043 subacaulis (Pursh) Rydb. (T. heterantha Small) I0044 taraxacifolia (Wats.) Heller

\section{Galpinsia}

(Oenothera in part)

ro045 Fendleri (A. Gray) Heller I0046 Greggii (A. Gray) Small I0047 Hartwegi (Benth.) Britton I0048 Toumeyi Small ro049 tubicula (A. Gray) Small

\section{Meriolix}

(Oenothera in part) roo5o serrulata (Nutt.) Walp. I005I spinulosa ('T. \& G.) Heller

\section{Eulobus}

I0052 Californicus Nutt.

\section{Sphaerostigma}

(Oenothera in part)

I0053 alyssoidès (H. \& A.) Walp.

I0054 macrophyllum Small

10055 minutiflorum(Wats)Small

I0056 andinum (Nutt.) Walp.

I0057 Bistorta (Nutt.) Walp.

roo58 Boothii (Dougl.) Walp.

I0059 campestre (Greene) Small I0060 minor Small

I0061 chamaenerioides (A. Gray) Small

I0030 brachycarpa (A.Gray) Britton IOO3I primiveris (A. Gray) Small roo32 triloba (Nutt.) Spach I0033 Watsoni Britton IOO34 Wrightii (A. Gray) Small

\section{Megapterium}

(Oenothera in part) roo35 dissectum )A. Gray) Small I0036 Fremonti (Wats.) Britton

I0062 contortum (Dougl.) Walp.

I0063 Greenei Small

10064 pubens (Wats.) Small I0065 decorticans (H. \& A.) Small I0066 Hilgardi (Greene) Small I0067 hirtellum (Greene) Small 10068 micranthum (Hornem.)Walp. roo69 Nelsoni Heller roo7o nitidum (Greene) Small 

I007 I refractum (Wats.) Small

I0072 rutila (Davidson) S. B. Parish I0073 spirale (Lehm.) Walp.

I0074 Utahense Small I0075 Veitchianum (Hook.) Small I0076 viridescens (Lehm.) Walp.

\section{Chylisma}

(Oenothera in part)

I0077 brevipes (A. Gray) Small I0078 cardiophylla (Torr.) Small I0079 cruciformis (Kellogg) Howell I0080 heterochroma (Wats.) Small I008I multijuga (Wats.) Small

roo82 Parryi (Wats.) Small I0083 pterosperma (Wats.) Small roo84 scapoidea (Nutt.) Small I0085 clavaeformis ('Torr.)Small

\section{Gayophytum}

I0086 caesium Nutt. I0087 eriospermum Coville I0088 diffusum T. \& G. I0089 lasiospernum Greene I0090 pumilum Wats. IO09I racemosum T. \& G. 10092 ramosissimum T. \& G. roo93 deflexum Hook.

\section{Gaura}

I0094 angustifolia Michx.

I0095 biennis L.

roog6 coccinea Pursh

I0097 integerrima Torr.

I0098 parvifolia T. \& G.

I0099 Drummondii T. \& G.

IOIOO filiformis Small

roror glabra Lehm.

IOIO2 Lindheimeri Engelm. \& Gray

roro3 longiflora Spach

IOIO4 macrocarpa Rothrock

IOIO5 Michauxii Spach

Ioro6 Nealleyi Coult.

Ioro7 Neo-Mexicana Wooton

roro8 parviflora Dougl.

IOIO9 sinuata Nutt.

IOI IO suffulta Engelm.

IOI I tripetala Cav.

Iorr2 villosa Torr.

\section{Stenosiphon}

roI 3 linifolium (Nutt.) Britton

(S. virgatus Spach)

\section{Heterogaura}

Ior 4 heterandra (Torr.) Coville (H. Californica Rothrock)

\section{Circaea}

IOII5 alpina $\mathrm{L}$.

IOI 6 Lutetiana L.

IOII7 Pacifica Aschers. \& Magnus

\section{HYDROCARYACEAE}

\section{Trapa}

IOII8 natans $\mathrm{L}$.

\section{HALORAGIDACEAE \\ Myriophyllum}

IOII9 alterniflorum DC.

IOI20 Farwellii Morong

IOI2I heterophyllum Michx.

IOI22 hippuroides Nutt.

Ior23 humile (Raf.) Morong (M. ambiguum Nutt.)

IOI24 laxum Shuttlw.

IOI 25 pinnatum (Walt.) B. S. P. (M. scabratum Michx.)

IOI 26 proserpinacoides Gill.

IOI 27 spicatum L.

IOI 28 tenellum Bigel.

Ior 29 verticillata $\mathrm{L}$.

\section{Proserpinaca}

IOI 30 palustris I. IOI3I pectinata Lam.

\section{Hippurus}

IOI 32 montana Ledeb.

IOI 33 tetraphylla L. f. IOI 34 vulgaris $\mathrm{L}$.

Ior35 fluviatilis Hart.

\section{ARALIACEAE \\ Echinopanax \\ (Fatsia) \\ IOI 36 horridum (Smith) Dec. \&,Pl.}

\section{Hedera}

Ior 37 Helix L. 



\section{Panax}

(Aralia in part)

ror $3^{8}$ quinquefolium L. IOI 39 trifolium L.

\section{Aralia}

Ior 40 Californica Wats.

ror4I acuminata Wats.

IOI 42 hispida Vent.

Ior 43 humilis Cav.

ror44 nudicaulis L.

ror45 elongata Nash

ror46 prolifera A. C. Apgar

IOI 47 racemosa L.

IOr48 spinosa L.

\section{UMBELLIFERAE}

\section{Hydrocotyle}

ror49 Americana L.

Ior 50 Bonariensis Lam.

Ior 51 Texana Coult. \& Rose IOI 52 Canbyi Coult. \& Rose

ror53 prolifera Kellogg

IOI 54 ranunculoides L. f.

IOI 55 umbellata L.

IOI56 verticillata Thunb.

\section{Centella}

Ior 57 Asiatica (L.) Urban (Hydrocotyle Asiatica L.)

\section{Bowlesia}

IOI 58 lobata R. \& P.

\section{Sanicula}

IOI 59 arctopoides H. \& A. ror6o bipinnata H. \& A. Ior6r bipinnatifida Dougl. Ior62 Canadensis L. Ior63 Floridana Bicknell Ior64 gregaria Bicknell ror65 Howellii Coult. \& Rose Ior66 laciniata H. \& A. Ior67 maritima Kellogg ror68 Marylandica L. Ior69 Menziesii H. \& A. Ior70 Nevadensis Wats. ror I S Smallii Bicknell Ior 72 trifoliata Bicknell Ior 73 tuberosa Torr.

\section{Eryngium}

IOI74 alismaefolium Greene

IOI75 aquaticum L.

(E. yuccaefolium Michx.)

Ior76 aristulatum Jepson

IOr 77 armatum (Wats.) C. \& R.

Ior 78 aromaticum Baldwin

IOI79 articulatum Hook.

IOI80 microcephalum C. \& R.

ror8r Baldwinii Spreng.

IOI82 diffusum Torr.

ror83 Floridanum C. \& R.

ror 84 foetidum $\mathrm{L}$.

ror85 Harknessii Curran

ror86 Hookeri Walp.

Ior87 Leavenworthii T. \& G.

Ior88 Lemmoni C. \& R.

ror 89 longifolium Cav.

rorgo Ludovicianum Morong

IorgI Mettaueri Wood

IOI92 nasturtifolium Juss.

IOI93 petiolatum Hook.

ror94 minimum C. \& R.

ror95 phyteumatos Delar.

roi 96 praealtum A. Gray

IOI97 prostratum Nutt.

Ior98 Ravenelii A. Gray

IOI99 virgatum Lam.

I0200 Vaseyi C. \& R.

IO2OI Virginianum Lam.

I0202 Wrightii A. Gray

\section{Chaerophyllum}

IO2O3 dasycarpum Nutt.

10204 procumbens (L.) Crantz

I0205 Shortii T. \& G.

I0206 Tainturieri Hook.

\section{Anthriscus}

I0207 Anthriscus (L.) Karst. (A. vulgaris Pers.)

ro208 Cerefolium (L.) Hoffm. ro209 sylvestris (L. Hoffm.

\section{Scandix}

IO2 Io Pecten-Veneris L.

\section{Washingtonia}

(Osmorrhiza)

I02 II brachypoda ('Torr.) Heller 

IO2I 2 Claytoni (Michx.) Britton (C. brevistylis DC.)

IO2I 3 divaricata Britton IO2I4 intermedia Rydb.

IO2I 5 longistylis ('Torr.) Britton I0216 nuda (Torr.) Heller

\section{Glycosma}

(Osmorrhiza in part)

IO2I7 ambigua A. Gray

ro2I8 occidentalis Nutt.

\section{Caucalis}

IO2I9 Anthriscus (L.) Huds. 10220 microcarpa H. \& A. IO22I nodosa (L.) Huds.

\section{Coriandrum}

ro222 sativum L.

\section{Anidrum}

(Bifora)

I0223 Americanum (DC.) Kuntze

\section{Apiastrum}

IO224 angustifolium Nutt. I0225 patens (Nutt.) C. \& R.

\section{Erigenia}

ro226 bulbosa (Michx.) Nutt.

\section{Orogenia}

10227 fusiformis Wats.

10228 Leibergii C. \& R.

I0229 linearifolia Wats.

\section{Conium}

I0230 maculatum L.

\section{Musenion}

I023r divaricatum (Pursh) Nutt.

ro232 Hookeri Nutt.

I0233 tenuifolium Nutt.

I0234 trachyspermum Nutt.

I0235 vaginatum Rydb.

\section{Velaea}

10236 arguta (T. \& G.) C. \& R.

I0237 ternata C. \& R.

I0238 glauca C. \& R.

I0239 Hartwegi (A. Gray) C. \& R.

I0240 Howellii C. \& R.
I024I Kelloggii (A. Gray) C. \& R. I0242 Parishii C. \& R.

I0243, vestita (Wats.) C. \& R.

\section{Hesperogenia}

10244 Stricklandi C. \& R.

\section{Museniopsis}

10245 Texana (A. Gray) C. \& R.

\section{Bupleurum}

10246 Americanuim C. \& R. I0247 rotundifolium L.

\section{Trepocarpus}

ro248 Aethusae Nutt.

\section{Apium}

I0249 graveolens I.

I0250 leptophyllum (DC.) F. Muell. I025I nodiflorum (L.) B. \& H.

\section{Petroselinum}

I0252 Petroselinum (L.)

\section{Spermolepis}

(Leptocaulis)

I0253 divaricatus (Walt, Britton I0254 echinatus (Nutt.) Heller

\section{Ammoselinum}

I0255 Butleri C. \& R. I0256 Popei 'T. \& G.

\section{Zizia}

I0257 aurea (L.) Koch

I0258 Bebbii (C. \& R.) Britton

IO259 cordata (Walt.) DC.

\section{Harbouria}

I0260 trachypleura (Wats.) C. \& R.

\section{Cicuta}

I026I Bolanderi Wats.

I0262 bulbifera L.

I0263 Californica A. Gray

I0264 maculata L.

I0265 angustifolia Wats.

10266 occidentalis Greene

I0267 purpurata Greene

I0268 vagans Greene 



\section{Deringa}

(Cryptotaenia)

I0269 Canadensis (L.) Kuntze

\section{Taeniopleurum}

I0270 Howellii C. \& R.

\section{Carum}

1027 I Carui L.

10272 Lemmoni C. \& R.

\section{Ataenia}

(Carum in part)

10273 Gairdneri H. \& A.

r0274 Kelloggii (A. Gray) Greene I0275 Oregana (Nutt.) Greene

\section{Aletes}

I0276 acaulis (Torr.) C. \& R.

\section{Leibergia}

I0277 orogenioides C. \& R.

\section{Taenidia}

I0278 integerrima (L.) Drude (Pimpinella integerrima

\section{Pimpinella}

I0279 apiodora A. Gray IO280 nudicaulis A. Gray

I028I Saxifraga I.

\section{Aegopodium}

I0282 Podagraria L.

\section{Eulophus}

$\mathrm{IO}_{2} 8_{3}$ Americanus Nutt.

I0284 Bolanderi (A. Gray) C. \& R. I0285 Californicus (A. Gray) C.\& R. I0286 Parishii C. \& R. I0287 Rusbyi C. \& R. I0288 Pringlei C. \& R. I0289 simplex C. \& R.

\section{Sium}

I0290 Carsoni Durand IO29I cicutifolium Gmel I0292 heterophyllum Greene

\section{Berula}

I0293 erecta (Huds.) Coville (B. angustifolia Koch)

\section{Ptilimnium}

(Discopleura)

10294 capillaceum (Michx.) Hollick ro295 laciniatum (Engelm. \& Gray)

Kuntze

10296 Nuttallii (DC.) Britton

\section{Cynosciadium}

10297 digitatum DC.

I0298 pinnatum (Nutt.) DC.

10299 pumilum Engelm.\& Gray

\section{Oenanthe}

I030o Californica Wats

IO3OI sarmentosa Presl

\section{Lilaeopsis}

\section{(Crantzia)}

10302 Carolinensis C. \& R.

IO3O3 lineata (Michx.) Greene

I03O4 occidentalis C. \& R.

I0305 Schaffineriana (Schlecht.)

\section{Aethusa}

C. \& R.

L.) 10306 Cynapium L.

Podistera

I0307 Nevadensis (A. Gray) Wats.

\section{Eurytaenia}

10308 Texana T. \& G.

\section{Foeniculum}

I0309 Foeniculum (L。) Karst. (F. vulgare Gaertn.)

\section{Selinum}

Iozio Benthami Wats.

IO3 I I capitellatum (A. Gray) B.\& H

IO3I 2 Dawsoni C. \& R.

IO3I3 eryngiifolium Greene

I03I4 Grayi Coult. \& Rose.

IO3I 5 Hookeri Wats.

I03I6 Kingii Wats.

IO3I7 Pacificum Wats.

\section{Ligusticum}

IO3I8 apiifolium (Nutt.) B. \& H.

ro3I9 Canadense (L.) Britton

(L. actaeifolium Michx.).

10320 Canbyi C. \& R. 

I032I Eastwoodiae C. \& R.

10322 filicinum Wats.

I0323 Grayi C. \& R.

10324 Macounii C. \& R.

I0325 scopulorum A. Gray

10326 Scoticum L.

10327 tenuifolium Wats.

IO328 verticillatum (Hook.) C. \& R.

\section{Coelopleurum}

I0329 Gmelini (DC.) Ledeb.

I0330 maritimum C. \& R.

\section{Oreoxis}

I033I humilis Raf.

\section{Thaspium}

10332 aureum Nutt.

I0333 barbinode (Michx.) Nutt.

ro334 pinnatifidum (Buckley)

A. Gray

ro335 trifoliatum (L.) Britton

\section{Conioselinum}

I0336 Chinense (L.) B. S. P. (C. Canadense T. \& G.)

\section{Angelica}

10337 ampla Aven Nelson

r0338 arguta Nutt.

ro339 atropurpurea L.

I0340 Breweri A. Gray

I034I Californica Jepson

I0342 Canbyi C. \& R.

ro343 Curtisii Buckley

I0344 Dawsoni Wats.

ro345 dentata (Chapm.) C. \& R.

I0346 genuflexa Nutt.

I0347 Hendersoni C. \& R.

ro348 leporina Wats.

IO349 lineariloba A. Gray

ro350 lucida L.

I035I Lyallii Wats.

I0352 pinnata Wats.

10353 Roseana Henderson

ro354 tomentosa Wats.

I0355 villosa (Walt.) B. S. P.

$$
\text { (A. hirsuta Muhl.) }
$$

I0356 Wheeleri Wats.

\section{Phellopterus}

I0357 littoralis Schmidt.

\section{Cymopterus}

10358 acaulis (Pursh) Rydb.

(C. glomeratus Raf.

I0359 bulbosus Aven Nelson

I0360 calcareus Jones

I036I campestris T. \& G.

I0362 cinerarius A. Gray

${ }_{10363}$ corrugatus Jones

I0364 decipiens Jones

I0365 Fendleri A. Gray

I0366 glaucus Nutt.

10367 globosus Wats.

I0368 Ibapensis Jones

I0369 Jonesii C. \& R.

I0370 lapidosus Jones

(Peucedanum lapidosum Jones)

ro37 I longipes Wats.

I0372 megacephalus Jones

I0373 montanus T. \& G.

I0374 pedunculatus Jones

I0375 purpurascens A. Gray

I0376 nivalis Wats.

ro377 Panamintensis C. \& R.

I0378 petraeus Jones

I0379 purpureus Wats

I0380 terebinthinus (Hook.) T.\& G.

IO38I Utahensis Jones

IO382 Eastwoodiae Jones

I0383 monocephalus Jones

\section{Coloptera}

ro384 Jonesii C. \& R.

I0385 Newberryi (Wats.) C. \& R. 10386 Parryi C. \& R.

\section{Pseudocymopterus}

10387 anisatus (A. Gray) C. \& R.

I0388 bipinnatus (Wats.) C. \& R. Io389 montanus (A. Gray) C. \& R. I0390 purpureus C. \& R.

I0391 tenuifolius(A.Gray)C.\&R.

\section{Polytaenia}

I0392 Nuttallii DC.

\section{Oxypolis}

\section{(Tiedemannia)}

I0393 Fendleri (A. Gray) Heller

ro394 filiformis (Walt.) Britton

( $T$. teretifolia $\mathrm{DC}$.) 

0395 rigidus (L.) Britton I0396 longifolius(Pursh)Britton 10397 ternata (Nutt.) Heller

\section{Leptotaenia}

10398 anomala C. \& R. I0399 Californica Nutt. ro400 dilatata Jepson IO4OI dissecta Nutt. 10402 Eatoni C. \& R. I0403 minor Rose I0404 multifida Nutt. I0405 purpurea (Wats.) C. \& R. I0406 Watsoni C. \& R.

\section{Peucedanum}

I0407 ambiguum Nutt. I0408 leptocarpum ('T. \& G.) ro409 Argense Jones C. \& R. ro4ro Austinae C. \& R. I04II bicolor Wats. rO4I 2 Brandegei C. \& R. I04I3 Californicum C. \& R. I04I4 Canbyi C. \& R. I04I5 caruifolium T. \& G. I04I 6 circumdatum Wats I04I7 confusum Piper I04I 8 Cons Wats I04I9 crittatum C. \& R. I0420 Cusickii Wats. IO42 I dasycarpum T. \& G. I0422 Donnellii C. \& R. I0423 erosim Jepson 10424 eurycarpum (A. Gray) C.\& R. I0425 Euryptera A. Gray I0426 evittatum C. \& R. r0427 farinosum Geyer ro428 foeniculaceum Nutt. I0429 Geyeri Wats. I0430 Gormani Howell I043I Grayi C. \& R. ro432 Hallii Wats. I0433 Hassei C. \& R. I0434 Hendersoni C. \& R. I0435 Howellii Wats. I0436 insulare Eastwood I0437 juniperinum Jones 10438 Kingii Wats. I0439 alpinum (Wats.) C. \& R. 10479 pusillus Michx.
I0440 laevigatum Nutt.

ro44I leiocarpum Nutt.

10442 Lemmoni C. \& R. I0443 macrocarpum Nutt. I0444 Martindalei C. \& R. I0445 angustum C. \& R. I0446 megarrhiza Aven Nelson I0447 microcarpum Howell 10448 Mohavense C. \& R. I0449 Nevadense Wats. I0450 cupulatum Jones I045I nudicaule (Pursh) Nutt.

ro452 Nuttallii Wats.

I0453 Oreganum C. \& R. I0454 Parishii C. \& R. I0455 Parryi Wats.

I0456 parvifolium T. \& G. I0457 Plummerae C. \& R. ro458 Pringlei C. \& R. ro459 robustum Jepson I0460 salmoniflorum C. \& R. I046I Sandbergii C. \& R. I0462 scopulorum Jones Io463 simplex Nutt. I0464 Suksdorfii Wats. ro465 Torreyi C. \& R. I0466 tomentosum Benth. I0467 triternatum Nutt. I0468 alatum C. \& R. I0469 brevifolium C. \& R. 10470 robustius C. \& $\mathrm{R}$. IO47I utriculatum Nutt.

I0472 Vaseyi C. \& R. IO473 villosum Nutt. 10474 Watsoni C. \& R.

\section{Imperatoria}

I0475 Ostruthium L.

\section{Pastinaca}

I0476 sativa L.

\section{Heracleum}

I0477 lanatum Michx.

\section{Daucus} I0478 Carota L. 



\section{CORNACEAE}

Garrya

I0480 buxifolia A. Gray

I0481 elliptica Dougl.

I0482 flavescens Wats.

I0483 Fremontii Torr.

ro484 ovata Benth.

I0485 Lindheimeri C. \& E.

I0486 Veatchii Kellogg

I0487 Wrightii Torr.

\section{Nyssa}

I0488 aquatica L.

$$
\text { (N. uniflora Wang.) }
$$

10489 biflora Walt.

ro490 Ogeche Marsh.

(N. capitata Walt.)

ro49r sylvatica Marsh.

(N. multiflora Wang.)

\section{Cornus}

I0492 alternifolia L. f.

I0493 Amonum Mill.

(C. sericea L.)

I0494 asperifolia Michx.

I0495 Baileyi Coult. \& Evans

10496 Californica C. A. Meyer

ro497 Canadensis L.

I0498 candidissima Marsh. (C. paniculata L'Her.)

I0499 circinata L'Her.

10500 Drummondii C. A. Meyer

IO5OI florida I.

I0502 glabrata Benth.

10503 Greenei Coult. \& Evans

I0504 microcarpa Nash

I0505 Nuttallii Audubon

I0506 occidentalis (T. \& G.) Coville

(C. pubescens Nutt.)

I0507 Purpusi Koehne

I0508 sessilis Torr.

I0509 stolonifera Michx.

IO5IO stricta Lam.

IO5II Suecia L.

IO5I 2 Torreyi Wats.

IO5I 3 Unalaskensis Ledeb.

\section{CLETHRACEAE}

\section{Clethra}

I05I4 acuminata Michx.
IO5I 5 alnifolia I.

I05I6 tomentosa Michx.

\section{PYROLACEAE}

\section{Chimaphila}

I05I7 maculata (L.) Pursh

I05I8 Menziesii Spreng.

I0519 umbellata (L.) Nutt.

\section{Pyrola}

I0520 aphylla Smith

IO52I asarifolia Michx.

10522 bracteata Hook.

I0523 chlorantha Sw.

I0524 occidentalis A. Gray

I0525 elliptica Nutt.

I0526 minor L.

ro527 oxypetala C. F. Austin

I0528 pallida Greene

I0529 picta Smith.

I0530 rotundifolia I.

IO53I incarnata DC.

I0532 pumila Hook.

I0533 secunda L.

I0534 uliginosa 'Torr.

\section{Moneses}

I0535 uniflora (L.) A. Gray

\section{MONOTROPACEAE}

\section{Allotropa}

I0536 virgata T. \& G.

\section{Monotropa}

I0537 fimbriata A. Gray

I0538 uniflora L.

\section{Hypopitys}

I0539 Hypopitys (L.) Small (Monotropa Hypopitys L.)

Pterospora

I0540 Andromedea Nutt.

Sarcodes

I054I sanguinea Torr.

\section{Monotropsis}

(Schweinitzia)

I0542 odorata Ell.

10543 Reynoldsiae (A. Gray) Heller 



\section{Pleuricospora}

I0544 fimbriolata A. Gray

\section{Hemitomes}

(Newberrya)

ro545 congestum A. Gray

I0546 pumilum Greene

I0547 spicatum (A. Gray) Heller

I0548 subterraneum (Eastwood)

Heller

\section{LENNOACEAE}

Pholisma

I0549 arenarium Nutt.

\section{Ammobroma}

I0550 Sonorae Torr.

\section{ERICACEAE}

\section{Elliottia}

ro55I racemosa Muhl.

\section{Cladothamnus}

I0552 campanulatus Greene

I0553 pyrolaeflorus Bong.

\section{Bejaria}

I0554 racemosa Vent.

\section{Ledum}

I0555 glandulosum Nutt. I0556 Groenlandicum Oeder (L. latifolium Ait.) I0557 palustre L. ro558 dilatatum Wahl.

\section{Rhododendron}

10559 Californicum Hook.

ro560 Catawbiense Michx.

I056r Chapmanii A. Gray

I0562 Kamtschaticum Pall.

10563 Lapponicum (L.) Wahl.

ro564 macrophyllum Don

I0565 maximum L.

10566 punctatum Andr.

10567 Vaseyi A. Gray

\section{Rhodora}

I0568 Canadensis L. (Rhododendron Rhodora Don)

Azaleastrum

I0569 albiflorum (Hook.) Rydb. (Rhododendron albiflorum Hook.)

\section{Azalea}

10570 arborescens Pursh

I057 I canescens Michx.

I0572 lutea L.

(A. calendulacea Michx.)

10573 nudiflora L.

I0574 occidentalis T. \& G.

I0575 viscosa $\mathrm{L}$.

ro576 glanca Michx.

I0577 hispida (Pursh) Britton

ro578 nitida (Pursh) Britton

\section{Menziesia}

I0579 glabella A. Gray

ro580 pilosa (Michx.) Pers.

(M. globularis Salisb.)

ro58I urceolaria Salisb.

(M. ferruginea Smith)

\section{Dendrium}

(Leiophyllum)

I0582 buxifolium (Berg.) Desv.

I0583 prostratum (Loud.) Ashe

\section{Chamaecistus}

(Loiseleuria)

I0584 procumbens (L.) Kuntze

\section{Kalmia}

ro585 angustifolia L.

I0586 cuneata Michx.

I0587 glauca Ait.

I0588 hirsuta Walt.

I0589 latifolia L.

I0590 myrtifolia Rand

I059I microphylla (Hook.) Heller

\section{Phyllodoce}

(Bryanthus)

I0592 Aleutica (Spreng.) Heller

I0593 Breweri (A. Gray) Heller

ro594 coerulea (L.) Gren. \& Godr.

I0595 empetriformis (Smith) Don

ro596 glanduliflora (Hook.) Rydb.

I0597 hybrida Rydb.

I0598 intermedia (Hook.) Rydb.

\section{Cassiope}

ro599 hypnoides (L.) D. Don ro60o lycopodioides D. Don 

Io6or Mertensiana (Bong.) D. Don I0602 Stelleriana DC.

I0603 tetragona (L.) D. Don

\section{Leucothoe}

10604 acuminata (Ait.) D. Don 10605 axillaris (Lam.) D. Don 10606 Catesbaei (Walt.) A. Gray 10607 Davisiae Torr. ro6o8 elongata Small ro6og racemosa (L.) A. Gray ro6ro recurva (Buckley) A. Gray

Andromeda

I06I I Polifolia L.

\section{Xolisma}

(Andromeda in part)

106I 2 ferruginea (Walt.) Heller Io6 I 3 fruticosa (Michx.) Nash I06I4 ligustrina (L.) Britton ro6I 5 foliosiflora (Michx.) Mohr

\section{Zenobia}

(Andromeda in part)

ro6r6 cassinefolia (Vent.) Pollard (A. speciosa Michx.)

I06I7 pulverulenta (Willd.) Pollard

\section{Pieris}

I06I 8 floribunda (Pursh) B. \& H.

I06I9 Mariana (L.) B. \& H.

I0620 nitida (Bartr.) B. \& H.

I062I phillyreifolia (Hook.) DC.

\section{Chamaedaphne}

(Cassandra)

ro622 calyculata (L.) Moench

\section{Oxydendron}

I0623 arboretım (L.) DC.

\section{Epigaea}

ro624 repens L.

\section{Gaultheria}

I0625 humifusa (Graham) Rydb. (G. Myrsinites Hook.)

I0626 ovatifolia A. Gray

r0627 procumbens $\mathrm{L}$.

I0628 Shallon Pursh

\section{Chiogenes}

ro629 hispidula (L.) T. \& G.

\section{Arbutus}

ro63o Arizonica (A. Gray) Sargent I063I Menziesii Pursh 10632 Xalapensis H. B. K.

\section{Aretostaphylos}

I0633 bicolor (Nutt.) Gray I0634 canescens Eastwood I0635 elegans Jepson I0636 glandulosa Eastwood ro637 glauca Lindl. I0638 Hookeri Don I0639 insularis Greene ro640 Manzanita Parry I064I montana Eastwood I0642 myrtifolia Parry 10643 Nevadensis A. Gray I0644 nummularia A. Gray ro645 Parryana Lemmon I0646 patula Greene I0647 polifolia H. B. K. I0648 pumila Nutt.

I0649 pungens H. B. K. ro650 Stanfordiana Parry ro65I tomentosa (Pursh) Dougl. ro652 Uva-ursi (L.) Spreng. I0653 alba Cockerell I0654 viscida Parry

\section{Mairania}

I0655 alpina (L.) Desv.

(Arctostaphylos alpina Spreng.)

\section{Gaylussacia}

I0656 brachycera (Michx.) A. Gray I0657 dumosa (Andr.) T. \& G. 10658 frondosa (L.) T. \& G. I0659 hirtella (Ait.) Klotzsch I0660 nana (A. Gray) Small Io66 I resinosa (Ait.) T. \& G. I0662 glaucocarpa Robinson I 0663 tomentosa Pursh I0664 ursina (M. A. Curtis) T. \& G.

\section{Vaccinium}

I0665 amoenum Ait.

ro666 atrococcum (A. Gray) Heller 10667 caesium Greene I0668 caespitosum Michx. 10669 arbuscula A. Gray 

I0670 cuneifolium Nutt. I067I Canadense Richards I0672 corymbosum L. ro673 crassifolium Andr. ro674 Elliottii Chapm. I0675 erythrococcuin Rydb.

(I. microphyllum (Hook.) Rydb. 10676 formosum Andr. I0677 fuscatum Ait. I0678 globulare Rydb. ro679 hirsutum Buckley ro680 membranaceum Dougl. I068I Myrsinites Lam. I0682 glaucum A. Gray I0683 Myrtillus L. I0684 nigrum (Wood) Britton I0685 nitidum Andr. I0686 occidentale A. Gray I0687 ovalifolium J. E. Smith I0688 ovatum Pursh ro689 pallidum Ait. I0690 parvifolium J. E. Smith ro69I Pennsylvanicum Lam. I0692 angustifolium (Ait.) ro693 salicinum Cham. A. Gray ro694 tenellum Ait. I0695 uliginosum $\mathrm{L}_{\text {. }}$ I0696 mucronatum Herder I0697 vacillans $\mathrm{Kalm}$ I0698 virgatum Ait. I0699 Vitis-Idaea I.

\section{Batodendron}

\section{(Vaccinum in part)}

I0700 arboteum (Marsh.) Nutt.

IO70I glaucescens Greene I0702 speciosum Greene

\section{Polycodium}

(Vaccinium in part)

I0703 caesium Greene I0704 elevatum (Banks \& Soland.)

Greene 10705 Floridanum (Nutt.) Greene ro706 oblongum Greene 10707 revolutum Greene I0708 stamineum (I, ) Greene

\section{Oxycoceus}

( accinium in part) I0709 erythrocarpus (Michx.) Pers. ro7 ro macrocarpus (Ait.) Pers. IO7I I Oxycoccus (L.) MacM.

\section{Calluna}

I07 12 vulgaris (L.) Salisb.

\section{Erica}

IO7I3 cinerea I. I07I4 Tetralix L.

\section{DIAPENSIACEAE \\ Diapensia}

IO7I5 Lapponica L.

\section{Pyxidanthera}

I0716 barbulata Michx.

\section{Shortia}

I07 I7 galacifolia T. \& G.

\section{Galax}

I07I8 aphylla L.

MYRSINACEAE

\section{Jacquinia}

IO7I9 armillaris L.

10720 pungens A. Gray

\section{Myrsine}

IO72I Rapanea C. \& S.

\section{Ieacora}

(Ardisia)

I0722 paniculata (Nutt.) Sudw. (A. Pickeringia T. \& G.)

\section{PRIMULACEAE}

\section{Primula}

10723 angustifolia Torr.

10724 borealis Duby

I0725 cuneifolia Ledeb.

ro726 Cusickiana A. Gray

10727 Egaliksensis Hornem.

I 0728 farinosa $\mathrm{I}_{1}$.

I0729 Maccalliana Wiegand

10730 Macounii Greene

1073I Mistassinica Michx. 

10732 mucronata Greene

10733 Arizonica Greene

10734 nivalis Pall.

I0735 Parryi A. Gray

10736 brachyantha Rydb.

10737 Rusbyi Greene

$1073^{8}$ serra Small

I0739 Sibirica Jacq.

I0740 suffrutescens A. Gray

I074I tenuis Small

\section{Douglasia}

I0742 arctica Hook.

I0743 biflora Aven Nelson

I0744 laevigata A. Gray

I0745 montana A. Gray

I0746 nivalis Lindl.

I0747 dentata A. Gray

\section{Androsace}

I0748 acuta Greene

I0749 arguta Greene

I0750 Arizonica Greene

I075I asprella Greene

I0752 capillaris Greene

ro753 Chamaejasne Host.

I0754 diffusa Small

I0755 filiformis Retz.

10756 Gormani Greene

I0757 occidentalis Pursh

I0758 pinetorum Greene

I0759 septentrionalis L.

10760 subulifera A. Gray

ro76r subumbellata (Aven Nelson)

\section{Hottonia}

I0762 inflata Ell.

\section{Samolus}

ro763 alyssoides Heller

I0764 cuneatus Small

I0765 ebracteatus H. B. K.

10766 floribundus H. B. K.

(S. Valerandi var. Americana)

\section{Lysimachia}

I0767 asperulaefolia Poir.

Io768 Fraseri Duby

10769 nummularia L.

I0770 polyantha Fernald. ro77 I producta (A. Gray) Fernald

ro772 punctata L.

I0773 quadrifolia L.

10774 terrestris (L.) B. S. P. (L. stricta Ait.)

I0775 vulgaris L.

\section{Steironema}

10776 ciliatum (L.) Raf.

I0777 lanceolatum (Walt.) A. Gray.

10778 angustifolium A. Gray

I0779 quadriflorum (Sims)

A. S. Hitchc.

I0780 radicans (Hook.) A. Gray

I078I tonsum (Wood) Bicknell

ro782 simplex Kearney

\section{Naumburgia}

I0783 thyrsiflora (L.) Duby

(Lysimachia thyrsiflora L.)

\section{Trientalis}

1o784 Americana (Pers.) Pursh I0785 arctica Fisch.

Io786 latifolia Torr.

\section{Glaux}

I0787 maritima I.

\section{Anagallis}

I0788 arvensis L.

Io789 coerulea Lam.

\section{Centunculus}

.

I079I pentandrus $\mathrm{R}$. Br.

\section{Dodecatheon}

I0792 acuminatum Rydb.

I0793 alpinum (A. Gray) Greene

I0794 Clevelandi Greene

I0795 conjugens Greene

I0796 cruciatum Greene

10797 Cusickii Greene

I0798 cylindrocarpum Rydb.

ro799 frigidum C. \& S.

I0800 glastifolium Greene

ro8or Hendersoni A. Gray

I0802 Hanseni Greene

Io803 Jeffreyi Moore 

I0804 Meadia L.

I0805 Bernalinum Greene

I0806 Frenchii Vasey

10807 gracile Greene

Io808 pauciflorum (Durand) Greene

I0809 monanthum Greene

I08Io puberulentum Heller

I08I I pubescens Rydb.

I08I 2 pulchrum Rydb.

I08I3 radicatum Greene

ro8r4 salinum Aven Nelson

I0815 tetrandrum Suksdorf

I08 I 6 uniflorum Rydb.

I08I7 viviparum Greene

\section{PLUMBAgINACEAE}

Plumbago

I08I8 scandens L.

Statice

I08I9 Armeria L.

\section{Limonium}

(Statice)

I0820 angustatum (A. Gray) Small I082 I Brasiliensis (Boiss.) Small I0822 Californicum (Boiss.) Small I0823 Carolinianum (Walt.) Britton I0824 limbatum Small I0825 Nashii Small

\section{SAPOTACEAE}

\section{Sideroxylon}

I0826 masticoodendron Jacq.

\section{Dipholis}

I0827 salicifolia (L.) A. DC.

\section{Bumelia}

I0828 angustifolia Nutt. I0829 cassinifolia Small I083o lanuginosa (Michx.) Pers. 1083I lucida Small I0832 lycioides (L.) Gaertn. f. I0833 megacocca Small I0834 microcarpa Small I0835 monticola Buckley I0836 reclinata Vent. I0837 rigida (A. Gray) Small ro838 rufotomentosum Small
I0839 tenax (L.) Willd. I0840 Texana Buckley

\section{Chrysophyllum}

I084I monopyrenum Sw. (C. oliviforme Lam.)

\section{Mimusops}

I0842 Sieberi A. DC:

EBENACEAE

\section{Diospyros}

I0843 Texana Scheele I0844 Virginiana L.

\section{SYMPLOCACEAE}

\section{Symplocos}

ro845 tinctoria (L.) L'Her.

\section{STYRACEAE}

\section{Mohrodendron}

\section{(Halesia)}

I0846 Carolinum (L.) Britton (H. tetraptera Ellis)

10847 dipterum (Ellis) Britton I0848 parviflorum (Michx.) Britton

\section{Styrax}

Io849 Americana Lam.

ro85. Californica Torr. 1085I grandifolia Ait. I0852 platanifolia Engelm. ro853 pulverulenta Michx.

\section{OLEACEAE}

\section{Fraxinus}

Io854 Americana L. 10855 anomala Torr. 10856 triphylla Jones 10857 Berlandieriana A. DC. I0858 Biltmoreana Beadle ro859 Caroliniana Mill. (F. platycarpa Michx.)

I0860 Curtissii Vasey ro86r cuspidata Torr. 10862 dipetala H. \& A. I0863 brachyptera A. Gray ro864 trifoliolata Torr. 10865 epiptera Michx. 

I0866 Greggii A. Gray ro867 lanceolata Borck. (F. viridis Michx.)

I0868 nigra Marsh. ( $F$. sambucifolia Lam.)

I0869 Oregana Nutt.

I0870 Pennsylvanica Marsh.

(F.pubescens Lam.)

ro87I profunda Bush

ro872 quadtangulata Michx.

I0873 Texensis (A. Gray) Sargent I0874 velutina Torr.

(F. pistaclaefolia Torr.)

\section{Syringa}

I0875 vulgaris $\mathrm{L}$.

\section{Hesperelaea}

10876 Palmeri A. Gray

\section{Osmanthus}

I0877 Americanus (L.) B. \& H.

\section{Adelia}

$$
\text { (Forestiera) }
$$

I0878 aeuminata Michx.

ro879 angustifolia (Torr.) Kuntze I0880 ligustrina Michx.

I088I Neo-Mexicana (A. Gray)

Kuntze

I0882 Arizonica (A.Gray) Heller I0883 porulosa Michx.

ro884 pubescens (Nutt.) Kuntze I0885 sphaerocarpa (Torr.) Kuntze ro886 reticulata ('Torr.) Kuntze

\section{Chionanthus}

I0887 Virginica L.

\section{Ligustrum}

ro888 vulgare $\mathrm{L}$.

\section{Menodora}

I0889 heterophylla Moricand I089o longiflora A. Gray I089I pubens A. Gray ro892 scabra A. Gray I0893 scoparia Engelm. I0894 spinescens A. Gray

\section{LOGA NACEAE}

\section{Gelsemium}

ro895 sempervirens (L.) Ait. f.

\section{Spigelia}

I0896 Anthelmia L.

I0897 gentianoides Chapm.

ro898 I.indheimeri A. Gray

10899 loganioides A. DC.

Iogoo Marylandica L.

Iogor Texana ('T. \& G.) A. DC.

\section{Cynoctonum}

(Mitreola)

I0902 angustifolium ('T.\& G.) Small

rogo3 Mitreola (L.) Britton (M. petiolata T. \& G.)

I0904 sessilifolia (Walt.) Gmel

\section{Polypremum}

rogo5 procumbens L.

\section{Emorya}

Iogo6 suaveolens Torr.

\section{Buddleia}

I0907 Hnmboldtiana R. \& S. I0908 marrubifolia Benth.

I0909 Pringlei A. Gray

rogio racemosa Torr.

IO9I I incana Torr.

IO9I 2 scordioides H. B. K.

I0913 Utahensis Coville

\section{GENTIANACEAE}

\section{Microcala}

Io9I4 quadrangularis(Willd.)Griseb

\section{Sabbatia}

IogI5 angularis (L.) Pursh

I09I6 angustifolia (Michx.) Britton (S. brachiata Ell.)

I09I7 Boykinii A. Gray

Iogr 8 calycina (Lam.) Heller

(S. calycosa Pursh)

Io9I9 campanulata (L.) Torr.

(S. gracilis Salisb.)

10920 campestris Nutt.

ro92 I dodecandra (L.) B. S. P.

(S. chloroides Pursh)

10922 stricta (A. Gray) Mohr.

10923 Elliottii Steud.

ro924 gentianoides E11.

ro925 lanceolata (Walt.) T. \& G. 

I0926 macrophylla Hook. ro927 paniculata (Michx.) Pursh I0928 stellaris Pursh

\section{Erythraea}

10929 Beyrichii T. \& G. I0930 calycosa Buckley I093I Arizonica A. Gray I0932 nana A. Gray ro933 Centaurium (L.) Pers. I0934 exaltata (Griseb.) Coville (E. Douglasii A. Gray)

I0935 floribunda Benth. I0936 Muhlenbergii Griseb. I0937 nudicaulis Engelm. I0938 Nuttallii Wats. ro939 pulchella (Sw.) Fries (E. ramosissima Pers.)

ro940 spicata (L.) Pers. I094I Texensis Griseb. I0942 trichantha Griseb. I0943 venusta A. Gray

\section{Bartonia}

I0944 iodandra Robinson \& Schk. I0945 verna (Michx.) Muhl. I0946 Virginica (L.) B. S. P. (B. tenella Muh1.)

\section{Obolaria}

I0947 Virginica L.

\section{Gentiana}

I0948 acuta Michx.

I0949 strictiflora Rydb.

I0950 affinis Griseb.

I095I Andrewsii Griseb

I0952 aniosepala Greene

I0953 arctophila Griseb.

ro954 aurea L.

ro955 auriculata Pall.

I0956 barbellata Engelm.

I0957 Bigelovii A. Gray

I0958 Californica - Kus. ro959 calycosa Griseb. ro960 monticola Rydb. rog6r connectens Pollard I0962 crinita Froel. I0963 decora Pollard
10964 detonsa Rottb. (G. serrata Gunner)

I0965 Douglasiana Bong. rog66 elegans Aven Nelson I0967 unicaulis Aven Nelson rog68 Elliottii Chapm.

rog69 flavida A. Gray ro970 Forwoodii A. Gray ro97 I frigida Haenke I0972 glanca Pall.

I0973 heterosepala Engelm. I0974 humilis Stev.

I0975 linearis Froel.

I0976 Menziesii Griseb. I0977 microcalyx Lemmon I0978 Newberryi A. Gray I0979 Oregana Engelm. I0980 Parryi Engelm. I098I platypetala Griseb. I0982 Porphyrio J. F. Gmel ( $G$. angustifolia Michx.)

I0983 propinqua Richards rog84 densiflora Griseb. rog8 5 prostrata Haenke I0986 puberula Michx. I0987 quinquefolia L.

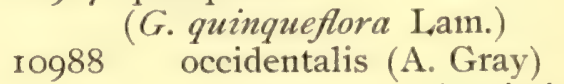
A. S. Hitchc. I0989 rubricaulis Schwein. I0990 Rusbyi Greene I099I Saponaria L. ro992 sceptrum Griseb. ro993 setigera A. Gray I0994 simplex A. Gray ro995 tenella Rottb. rog96 tortuosa Jones I0997 ventricosa Griseb. ro998 villosa $\mathrm{I}_{\text {. }}$. (G. ochroleuca Froel.)

I0999 Wislizeni Engelm.

I 1000 Wrightii A. Gray

\section{Pleurogyne}

I IooI Carinthiaca (Wulf.) Griseb. I 1002 rotata (L.) Griseb.

\section{Swertia}

I IOO 3 perennis I. 



\section{Frasera}

I I004 albicaulis Doug1.

I 1005 albomarginata Wats.

I 1006 caerulea Mulford

I 1007 Carolinensis Walt.

I 1008 Cusickii A. Gray

I I009 fastigiata (Pursh) Heller

( $F$. thyrsiflora Hook.)

I Ioro montana Mulford

I IOI I nitida Benth.

IIOI2 paniculata Torr.

I IOI 3 Parryi Torr.

I ror 4 speciosa Dougl.

I IOI 5 scabra Jones

I IOI6 tubulosa Coville

I IOI 7 Utahensis Jones

\section{Tetragonanthus}

(Halenia)

I Ior 8 Brentonianus (Griseb.) Heller

I Iorg deflexus (J. E. Smith) Kuntze I 1020 heterantherus (Griseb.)Heller IIO2I Rothrockii (A. Gray) Heller

\section{Eustoma}

I 1022 exaltata (L.) Griseb.

I 1023 Russellianum (L.) Griseb.

\section{Voyria}

I I024 Mexicana Griseb.

\section{MENYANTHACEAE}

\section{Menyanthes}

I 1025 trifoliata L.

\section{Nephrophyllidium}

(Menyanthes in part)

I 1026 Crista-galli (Menzies) Gilg.

\section{Limnanthemum}

I 1027 aquaticum (Walt.) Britton (L. trachyspermum A. Gray) I 1028 lacunosum (Vent.) Griseb. I 1029 nymphaeoides (L.) H. \& L.

\section{APOCYNACEAE}

\section{Haplophyton}

I IO3O cimicidum A. DC.

\section{Amsonia}

Iro3 I Amsonia (L.) Britton (A. Tabernaemontana
I I032 brevifolia A. Gray

I IO33 ciliata Walt.

(A. angustifolia Michx.)

Iro34 longiflora Torr.

I 1035 Palmeri A. Gray

I I036 Texana (A. Gray) Heller

I İO37 tomentosa Torr.

\section{Vinca}

IIO38 minor L.

\section{Lochnera}

I I039 rosea (L.) Reichb. (Vinca rosea L.)

\section{- Vallesia}

I 1040 glabra Cav.

\section{Echites}

Iro4I Andrewsii Chapm.

I 1042 paludosa Vahl

I 1043 Sagraei A. DC.

Iro44 umbellata Jacq.

\section{Macrosiphonia}

I I045 brachysiphon (Torr.) A. Gray I ro46 macrosiphon (Torr.) Heller (M. Berlandieri A. Gray)

I 1047 Wrightii A. Gray

\section{Cycladenia}

I 1048 humilis Benth.

I I049 tomentosa A. Gray

\section{Secondatia}

(Trachelospermum)

I 1050 difformis (Walt.) Benth.

\section{Urechites}

I I05I suberecta (Jacq.) Muell. Arg.

\section{Аросупum}

I I052 album Greene

I 1053 androsaemifolium L.

I I054 cannabinum L.

II055 glaberrimum A. DC.

I 1056 hypericifolium 'Ait.

I 1057 medium Greene

I 1058 nemorale G. S. Miller

I 1059 pubescens R. Br.

I 1060 pumilum (A. Gray) Greene I 106 I speciosum G. S. Miller 

I I062 urceolifer G. S. Miller

\section{Nerium}

I 1063 Oleander L.

\section{ASCLEPIADACEAE \\ Periploca}

I 1064 Graeca L.

\section{Astephanus}

I 1065 Utahensis Engelm.

\section{Philibertella}

(Philibertia)

I 1066 clausa (Jacq.) Vail ( $P$. viminalis A. Gray)

I 1067 crispa (Benth.) Vail

(P. undulata A. Gray)

I 1068 cynanchoides (Dec.) Vail

r 1069 Hartwegi Vail

\section{(P. linearis A. Grey)}

I 1070 heterophylla (Engelm.)

Vail

IIO7I hirtella (A. Gray) Vail Iro72 Torreyi (A. Gray) Vail

\section{Podostigma}

I 1073 pubescens Ell.

\section{Gomphocarpus}

II074 cordifolius Benth.

II075 hypoleucus A. Gray

I 1076 tomentosus (Torr.) A. Gray

I 1077 Xanti A. Gray

\section{Schizonotus}

I ro78 purpurascens A. Gray

\section{Asclepias}

r ro79 albicans Wats.

I Io80 amplexicaulis Michx.

IIO8I arenaria Torr.

II082 brachystephana Engelm.

I I083 cinerea Walt.

I I084 cryptoceras Wats.

I 1085 Curassavica L.

I 1086 Curtissii A. Gray

I 1087 decumbens L.

I 1088 ecornuta Kellogg

II089 elata Benth.

I I090 eriocarpa Benth.
I IogI erosa Torr.

I 1092 obtusa A. Gray

r 1093 exaltata (L.) Muhi. (A. phytolaccoides Pursh)

I 1094 Feayi Chapm.

I 1095 Fremontii 'Torr.

I I096 galioides H. B. K.

I 1097 glaucescens H. B. K.

I I098 Hallii A. Gray

I I099 incarnata L.

II 100 longifolia $\mathrm{L}$.

IIIOI involucrata Engelm.

I I 102 labriformis Jones

IIIO3 lanceolata Walt. (A. paupercula Michx.)

IIIO4 latifolia (Torr.) Raf. (A. Jamesii Torr.)

III05 Lemmoni A. Gray

IIIo6 linearis Scheele

III07 linifolia H. B. K.

I I 108 macrosperma Eastwood

I I 109 macrotis Torr.

IIIIO Meadii Torr.

I I I I Mexicana Cav.

IIII2 Michauxii Dec.

I III 3 nummularia Torr.

IIII4 obovata Ell.

IIII5 obtusifolia Michx.

IIII6 ovalifolia Dec.

I I I 7 parvula (A. Gray) Vail

I I I 8 perennis Walt.

IIII9 pulchra Ehrh.

I I 20 pumila (A. Gray) Vail

I I 2 I purpurascens L.

I I 22 quadrifolia Jacq.

III23 quinquedentata A. Gray

III 24 Neo-Nexicana Greene

III 25 rubra L.

II 26 scaposa Vail

I I 27 Simpsoni Chapin.

I I 28 speciosa 'Torr.

I I 29 subulata Dec.

II 30 subverticillata (A. Gray) Vail

III3I Sullivantii Engelm.

I I 32 Syriaca L.

(A. Cormuti Dec.)

I I 33 Texana Heller

III34 tomentosa E11. 

I II 35 tuberosa L.

I I 36 uncialis Greene

III37 variegata L.

I I 38 verticillata $L$.

I I 39 vestita H. \& A.

III40 Mohavensis A. Gray

I I 4I viridula Chapm.

\section{Podostemma}

(Asclepias in part)

III42 Emoryi Greene

I I 43 Helleri Greene

I I 44 Lindheimeri(Engelm.\&Gray)

Greene

I I 45 longicornu (Benth.) Greene I I 46 nyctaginifolium (A. Gray)

\section{Acerates}

I I 47 angustifolia (Nutt.) Dec.

(Asclepias stenophyllus A. Gray)

III48 auriculata Engelm.

I I 49 bifida Rusby

I I 50 Floridana (Lam.) A.S.Hitchc. (A. longifolia Ell.

III5I lanuginosa Dec.

III52 Rusbyi Vail

I I 53 viridiflora (Raf.) Eaton

I I 54 Ivesii Britton

(A. viridiflora var. lanceolata)

II 55 linearis A. Gray

\section{Anantherix}

I I 56 connivens (Baldwin) A. Gray

\section{Asclepiodora}

I I 57 decumbens (Nutt.) A. Gray

II 58 viridis (Walt.) A. Gray

II 59 angustior A. Gray

\section{Metastelma}

I I 60 Arizonicum A. Gray

I I 6I Bahamense Griseb.

I I 62 barbigerum Scheele

I I 63 Blodgettii A. Gray

II 64 Palmeri Wats.

III65 Reverchoni A. Gray

\section{Gonolobus}

(Ampelenus; Enslenia)

I I 66 laevis Michx. (Enslenia albida Nutt.)

\section{Pattalias}

(Melinia)

I I 67 angustifolius (Torr.) Wats.

\section{Cynanchum}

(Vincetoxicum Moench, not Walt.)

I I 68 nigrum (L.) Pers.

II 69 palustre (Pursh) Heller

I I 70 scoparium Nutt.

\section{Roulinia}

II 7 I unifaria (Scheele) Torr.

\section{Rothrockia}

II 72 cordifolia A. Gray

\section{Vincetoxicum}

(Gonolobus of authors, not Michx.)

I I 73 Baldwinianum(Sweet)Britton

I I 74 biflorum (Raf.) Heller

I I 75 Carolinense (Jacq.) Britton

I I 76 cynanchoides

(Engelm. \& Gray) Heller

I I 77 flavidulum (Chapm.) Heller

II 78 Floridanum Vail

I I 79 gonocarpos Walt.

(G. macrophyllus Willd.)

I I 80 hastulatum (A. Gray) Heller

I I 8I hirsutum (Michx.) Britton

II 82 obliquum (Jacq.) Britton

II 83 parviflorum ('Torr.) Heller

I I 84 parvifolium (Torr.) Heller

I I 85 productum ('Torr.) Vail

I I 86 pubiflorum (Dec.) Heller

I I 87 reticulatum (Engelm.) Heller

I I 88 sagittifolium (A.Gray) Heller

II 89 Shortii (A. Gray) Britton

I I I 90 suberosum (L.) Britton

I I I9I (Wrightii (A. Gray) Heller

\section{Pherotrichis}

I I 92 Schaffneri A. Gray

\section{Lachmostoma}

III93 Arizonicum A. Gray

\section{CONVOLVULACEAE}

\section{Dichondra}

I I 94 argentea Willd.

I I 195 evolvulacea (L. f.) Britton

(D. repens Forst.) 



\section{Breweria}

I I 196 angustifolia Nash

I I 97 aquatica (Walt.) A. Gray

I I 98 grandiflora A. Gray

I I 99 humistrata (Walt.) A. Gray

I 200 ovalifolia (Torr.) A. Gray

I 20 I Pickeringii (M. A. Curtis)

I 202 villosa Nash

A. Gray

\section{Cressa}

I 203 aphylla Heller

I 204 Truxillensis H. B. K.

\section{Evolvulus}

I 205 alsinoides L.

II 206 Arizonicus A. Gray

I 207 discolor Benth.

I 208 laetus A. Gray

I 209 linifolius L.

II 210 mollis Small

II 2 I I mucronatus Sw.

II 2 I 2 Nuttalianus R. \& S.

I 2 I 3 pilosus Nutt.

II2I4 sericeus Sw.

\section{Quamoclit}

(Ipomoea in part)

I 215 coccinea (L.) Moench

I 2 I 6 hederifolia (L.) Chois.

I I2 7 Quamoclit (L.) Britton

\section{Ipomoea}

II 2 I 8 barbatisepala A. Gray

I 2 I9 barbigera (Don) Sweet

r1220 Bona-nox. L.

I 22 I capillacea Don

I 222 patens A. Gray

I 223 cardiophylla A. Gray

I 224 Carletoni Holzinger

I 225 Carolina (L.) Pursh

(I. trichocarpa El1., and 1. commutata $\mathrm{R}$. \& S.)

I 226 carnosa $\mathrm{R}$. Br.

I 1227 cathartica Poir.

I 228 costellata Torr.

I 229 cuneifolia A. Gray

I 230 fiistulosa Mart.

I 23 I hederacea (L.) Jacq.

I 232 integriuscula A. Gray

I 233 Jalapa (L.) Pursh
I 234 lacunosa L.

I 235 Lemmoni A. Gray

I 236 leptophylla Torr.

I 237 leptotoma Torr.

I 238 Lindheimeri A. Gray'

I 239 longifolia Benth.

I 240 Mexicana A. Gray

I 24 I palmata Forsk.

I 242 pandurata (L.) Meyer

I 243 pes-caprae (L.) Sweet

I 244 Plummerae A. Gray

I 245 purpurea (L.) Roth

I 246 sagittata Cav.

I 247 sinuata Ortega

I 248 tenuiloba Torr.

I 249 Thurberi A. Gray

I 250 trifida Berlandieri A. Gray

I 25 I Torreyana A. Gray

I 252 triloba $\mathrm{L}$.

I 253 Tuba Don

I 254 Wrightii A. Gray

\section{Jacquemontia}

Ir255 Curtissii Peter

I 256 Pringlei A. Gray

I 257 tamnifolia (L.) Griseb.

I $25^{8}$ violacea Choisey

\section{Convolvulus}

II 259 Americanus (Sims) Greene

I 260 aridus Greene

II 26I arvensis $\mathrm{L}$.

I 262 Binghamiae Greene

I 263 canporum Greene

I 264 collinus Greene

I 265 deltoidens Greene

I 266 fruticetorum Greene

I 267 fulcratus (A. Gray) Greene

I 268 gracilentus Greene

I 269 Havanensis Jacq.

I 270 hermannioides A. Gray

II27I incanus Vahl

I 272 Japonicus Thunb.

I 273 limnophilus Greene

I 274 longipes Wats.

I 275 luteolus A. Gray

I 276 Macounii Greene

I 277 macrostegius Greene

I 278 malacophyllus Greene

(C. villosus A. Gray) 

II279 Nuttallii Greene

II 280 nyctagineus Greene

II 281 occidentalis A. Gray

II 282 tenuissimus A. Gray

II 283 pentapetaloides L.

II 284 polymorphus Greene

Ir285 purpuratus Greene

I 286 repens L.

II 287 sepium L.

I 288 Soldanella L.

II 289 spithamaeus L.

II 290 subacaulis (H. \& A.) Greene

(C. Californicus Chois.)

Ir29I tomentellus Greene

\section{CUSCUTACEAE}

\section{Cuscuta}

II 292 aphylla Raf.

(C. glomerata Chois.)

I 293 applanata Engelm.

I 294 arvensis Beyrich

II 295 Californica Choisy

II 296 breviflora Engelm.

I 297 longiloba Engelm.

I 298 Cephalanthi Engelm.

(C. tenuiflora Engelm.)

I 299 compacta Juss.

I 300 Coryli Engelm.

(C. inflexa Engelm.)

II 301 cuspidata Eingelm.

II 302 denticulata Engelm.

II 303 epilinum Weihe

II 304 epithymum Murr.

II 305 exaltata Engelm.

II 306 Gronovii Willd.

II307 calyptrata Engelm.

II 308 curta Engelm.

II 309 latiflora Engelm.

II 3 Io indecora Choisy

II3II leptantha Engelm.

II 3 I 2 obtusiflora glandulosaEngelm

II 3 I3 neuropetala Engelm.

II 3I4 odontolepis Engelm.

II3I5 Plattensis Aven Nelson

II3I6 Polygonorum Engelm.

(C. chlorocarpa Engelm.)

II 3 I 7 racemosa Chiliana Engelm.

II 3 I 8 rostrata Shuttlw.
II3I9 salina Engelm.

I 320 squamata Engelm.

I 32 I subinclusa Dur. \& Hilg.

II 322 umbellata H. B. K.

\section{POLEMONIACEAE}

Phlox

Ir 323 adsurgens Torr.

II 324 albomarginata Iones

II 325 minor Jones

I 326 alyssifolia Greene

Ir 327 amoena Sims

Ir 328 andicola Nutt.

II 329 aspera Elias Nelson

II 330 austromontana Coville

II33I prostrata Elias Nelson

Ir 332 bifida Beck

II 333 Brittonii Small

I 334 bryoides Nutt.

II335 caespitosa Nutt.

II 336 canescens T. \& G.

I 337 cernua Elias Nelson

II338 collina Rydb.

I 339 condensata (A. Gray)

Elias Nelson

I 340 Hendersoni Elias Nelson

II 34 I costata Rydb.

I 342 Covillei Elias Nelson

II 343 diapensioides Rydb.

I 344 diffusa Benth.

II 345 divaricata L.

II 346 dolicantha A. Gray

Ir 347 Douglasii Hook.

II 348 Drummondii Hook.

I 349 villosissima A. Gray

II350 Floridana Benth.

II 35 I glaberrima L.

II $35^{2}$ suffruticosa A. Gray

I 353 gladiformis (Jones)

Elias Nelson

II354 hirsuta Elias Nelson

I 355 Hoodii Richards

I 356 glabrata Elias Nelson

II357 Kelsyi Britton

I 358 lanceolata Elias Nelson

II359 linearifolia (Hook.) A. Gray

II 360 longifolia Nutt.

II 36I pubera Elias Nelson 

I 362 maculata L.

II 363 candida Michx.

I 364 multiflora Aven Nelson

I 365 depressa Elias Nelson

I 366 muscoides Nutt.

II 367 nana Nutt.

II 368 occidentalis Durand

II 369 ovata L.

I 370 paniculata L.

I 37 I pilosa L.

I 372 detonsa A. Gray

I 373 Piperi Elias Nelson

I 374 reptans Michx.

II 375 Richardsonii Hook.

II 376 rigida Benth.

II 377 Roemeriana Scheele

Ir 378 Sabini Dougl.

II379 scleranthifolia Rydb.

I 380 Sibirica L.

II 38 I speciosa Pursh

I 382 Stansburyi ('Torr.) Heller

I $3^{8} 3$ brevifolia (A.Gray) Rydb.

II 384 Stellaria L.

I 385 subuluta L.

I $3^{86}$ tenuifolia Elias Nelson

I 387 tenuis (A. Gray) Elias Nelson

II 388 triovulata Thurber

II 389 virida Elias Nelson

I 390 viscida Elias Nelson

II 39I Whitedii Elias Nelson

I 392 Woodhousei (A. Gray) Torr.

\section{Collomia}

(Gilia in part)

II 393 aristella (A. Gray) Rydb.

II 394 debilis (Wats.) Greene

I 395 diversifolia Greene

I 396 grandiflora Dougl.

II 397 heterophylla Hook.

rI398 linearis Nutt.

II 399 Mazama Coville

I 400 Rawsoniana Greene

I 4 OO tenella A. Gray

II $4 \mathrm{O} 2$ tinctoria Kellogg
(G. linearis var. subulata A. Gray)

Microsteris

I 403 Californica Greene

II 404 diffusa Heller
Ir405 glabella Greenie

I 406 gracilis (Dougl.) Greene (Collomia gracilis Dougl.)

I 407 humilis (Dougl.) Greene

I 408 MacDougalii Heller

I 409 micrantha (Kellogg) Greene

I I4IO stricta Greene

\section{Gilia}

II4II abrotanifolia Nutt.

I 4 I 2 aggregata (Pursh) Spreng.

I 4 I3 Bridgesii A. Gray

II4I4 attenuata (A. Gray)

I I45 caespitosa (Nutt.)

Aven Nelson

I I4I6 calcarea Jones

I 4 I 7 Californica Benth.

I 4 I 8 campanulata A. Gray

II4I9 capillaris Kellogg

I 420 capitata Dougl.

I I42I cephaloidea Rydb.

II422 Chamissonis Greene

I 423 Columbiana Piper

I 424 congesta Hook.

I 425 crebrifolia A. Gray

I 426 paniculata Jones

I 427 debilis Wats.

I 428 densiflora Benth.

I 429 densifolia Benth.

II430 elongata A. Gray

I I43 I depressa Jones

I 432 filifolia Nutt.

Ir433 diffusa A. Gray

I 434 filiformis Parry

I 435 floccosa A. Gray

I 436 floribunda A. Gray

II437 arida Jones

I $43^{8}$ gilioides (Benth.) Greene

I 439 glutinosa (Benth.) A. Gray

I 440 Grayi Aven Nelson

(G. caespitosa A. Gray)

II44I Gunnisoni T. \& G.

I 442 Hallii S. B. Parish

I 443 Havardi A. Gray

I 1444 Haydeni A. Gray

I 445 hispida Piper

I 446 Howardi Jones 

I 447 iberidifolia Benth.

I 448 incisa Benth.

I 449 inconspicua (J. E. Smith)

Sweet II 496 floribunda A. Gray

Ir $45^{\circ}$ sinuata A. Gray

II45I latiflora A. Gray

II $45^{2}$ cana Jones

I 453 exilis A. Gray

I 454 latifolia Wats.

II455 laxiflora (Coult.) Osterhout

II 456 leptalea (A. Gray) Greene

II457 leptomeria A. Gray

II $45^{8}$ tridentata Jones

II459 longiflora ('Torr.) Don

I 460 lutescens Steud.

I 46I Macombii Torr.

II 462 maculata S. B. Parish

II 463 McVickerae Jones

II 464 micromeria A. Gray

I 465 minutiflora Benth.

II466 multicaulis Benth.

II467 tenera A. Gray

I 468 multiflora Nutt.

II 469 cuspidata A. Gray

I 470 Nevinii A. Gray

I 47 I Nuttallii A. Gray

I1 472 ochroleuca Jones

I 4773 pentstemonoides Jones

I 474 pinnatifida Nutt.

II 475 polycladon Torr.

II476 pumila Nutt.

II 477 pungens (Torr.) Benth.

I 478 Hookeri A. Gray

I 479 rigidula Benth.

I 480 acerosa A. Gray

II48I rubra (L.) Heller

(G. coronopifolia Pers.)

I 482 sedifolia T. S. Brandegee I 483 spicata Nutt.

II 484 deserta Aven Nelson

II 485 staminea Greene

I 486 stenothyrsa A. Gray

I 487 subnuda 'Torr.

I 488 tenerrima A. Gray

I 489 tenuiflora Benth.

I 490 altissima S. B. Parish

Ir49I tenuiloba S. B. Parish

11492 Thurberi A. Gray
I 493 tricolor Benth.

I I 494 Triodon Eastwood.

II495 virgata Steud.

I 497 Watsoni A. Gray

I 498 Wrightii A. Gray

\section{Gymnosteris}

(Gilia in part)

I 499 nudicaulis (H. \& A.) Greene

I 500 pulchella Greene

II 50r parvula (Rydb.) Heller

\section{Navarretia}

(Gilia in part)

I 502 atractyloides H. \& A.

I 503 Breweri (A. Gray) Greene

I 504 cotulaefolia H. \& A.

I 505 divaricata (Torr.) Greene

II 506 filicaulis ('T. \& G.) Greene

II 507 foliacea Greene

II 508 hamata Greene

I 509 heterodoxa Greene

I 5 IO intertexta Hook.

I25II leptantha Greene

II 5I2 leucocephala Benth.

I 5'3 mellita Greene

II5I4 minima Nutt.

I 5 I5 mitrocarpa Greene

I 5 I6 nigellaeformis Greene

I $5^{17} 7$ parvula Greene

II $5^{1} 8$ prolifera Greene

I 5 I9 prostrata (A. Gray) Greene

II 520 pubescens H. \& A.

I 52 I setiloba Coville

II 522 squartosa H. \& A.

I 523 subuligera Greene

I 524 tagetina Greene

II 525 viscidula Benth.

\section{Linanthus}

(Gilia in part)

I 526 acicularis Greene

I 527 ambiguus (Rattan) Greene

I 528 androsaceus (Benth.) Greene

II529 aureus (Nutt.) Greene

I 530 bicolor (Nutt.) Greene

II 53I Bigelovii (A. Gray) Greene

I 532 Bolanderi (A. Gray) Greene 

I 533 breviculus (A. Gray) Greene

I 534 ciliatus (Benth.) Greene

I 535 demissus (A. Gray) Greene

II 536 dianthiflorus Greene

I 537 dichotomus Benth.

I $53^{8}$ filipes (Benth.) Greene

I 539 grandiflorus (Benth.) Greene

I 540 Harknessi (Curran) Greene

I 54 I Jonesii (A. Gray) Greene

I 542 Lemmoni (A. Gray) Greene

I 543 liniflorus (Benth.) Greene

II544 luteolus Greene

I 545 montanus Greene

I 546 neglectus Greene

I 547 nudatus Greene

I 548 Parryae (Benth.) Greene

I 549 parviflorus (Benth.) Greene (Gilia micrantha Steud.)

II $55^{\circ}$ pharnaceoides(Benth.)Greene

I I 55I pusillus (Benth.) Greene

I 552 Rattani (A. Gray) Greene

I 553 rosaceus (Hook.) Greene

I 554 serrulatus Greene

\section{Langloisia}

(Gilia in part)

I 555 Matthewsii (A. Gray) Greene

I 556 Schottii (Torr.) Greene

I 557 setosissima ('T. \& G.) Greene

\section{Polemonium}

I $55^{8}$ bicolor Greenman

I 559 Brandegei (A. Gray) Greene (Gilia Brandegei A. Gray)

I 560 caeruleum L.

I 56 I carneum A. Gray

I 562 luteum A. Gray

I 563 elegans Greene

I 564 eximius Greene

I 565 filicinum Greene

I 566 flavum Greene

Ir 567 foliosissimum A. Gray

I 568 Haydeni Aven Nelson

I 569 humile Willd.

II 570 mellitum (A. Gray)

Aven Nelson

II57 I micranthum Benth.

I 572 occidentale Greene

I 573 parvifolium Nutt.
I 574 pectinatum Greene

I 575 pulchellum Bunge

I 576 reptans L.

I 577 Van-Bruntiae Britton

I 578 viscosum Nutt.

I 579 pilosum Greenman

\section{Loeselia}

I 580 glandulosa Don

\section{HYDROPHYLLACEAE}

\section{Hydrophyllum}

II58I albifrons Heller

I $5^{82}$ appendiculatum Michx.

I 583 Canadense L.

I $5^{84}$ capitatum Dougl.

I $5^{85}$ alpinum Wats.

I 586 congestum Wiegand

II 587 Fendleri (A. Gray) Heller

I 588 macrophyllum Nutt.

II 589 occidentale (Wats.) A. Gray

II 590 Watsoni A. Gray

I 59 I tenuipes Heller

I 592 Virginicum L.

\section{Nemophila}

I 593 aurita Lindl.

I 594 breviflora A. Gray

II 595 insignis Dougl.

I 596 intermedia Bioletti

I 597 maculata Benth.

I 598 membranacea (Benth.)Greene

I 599 Menziesii H. \& A.

I 600 integrifolia S. B. Parish

II6or microcalyx (Nutt.) F. \& M.

I 602 parviflora Dougl.

I 603 phacelioides Nutt.

I 604 racemosa Nutt.

I 605 sepulta S. B. Parish

I 606 spathulata Coville

\section{Macrocalyx}

(Ellisia)

I 607 micranthus (Torr.) Coville (Phacelia micrantha Torr.)

I 608 Nyctelea (L.) Kuntze

\section{Euerypta}

(Ellisia in part)

I 609 chrysanthemifolia (Benth.) 

II610 paniculata Nutt.

I 6I I Torreyi (A. Gray) Heller

\section{Draperia}

II6I2 systyla Torr.

\section{Phacelia}

I16r3 alpina Rydb.

I 614 Arizonica A. Gray

I16I5 Arthuri Greene

I 6 16 Bicknellii Small

I 6 I 7 bicolor Torr.

I 6 I 8 biennis Aven Nelson

I16rg bipinnatifida Michx.

I 620 Bolanderi A. Gray

I 62 I Boykinii (A. Gray) Small

I 622 brachyloba A. Gray

II 623 brevistylis Buckley

II624 Breweri A. Gray

II 625 caerulea Greene

I 626 campanularia A. Gray

I 62.7 campestris Aven Nelson

11628 cephalotes A. Gray

I 629 ciliata Benth.

I 630 circinatiformis A. Gray

II63I congesta Hook.

I 632 dissecta A. Gray

I 633 Covillei Wats.

II634 crassifolia Torr.

I 635 crenulata Torr.

II636 curvipes Torr.

I 637 Davidsonii A. Gray

I 638 macrantha S. B. Parish

I 639 demissa A. Gray

I I640 deserta Aven Nelson

II64I distans Benth.

I 642 divaricata A. Gray

I 643 Douglasii Torr.

1 I644 dubia (L.) Small (P. parviflora Pursh)

I 645 Eisenii Brandegee II646 fimbriata Michx.

II647 floribunda Greene

I 648 Franklinii (R: Br.) A. Gray

I 649 Fremontii Torr.

I 650 frigida Greene

I 65 I glabra Nutt.

I 652 glandulosa Nutt.

I 653 glechomaefolia A. Gray
I 654 grandiflora A. Gray

I 655 grisea A. Gray

I 656 gymnoclada Torr.

I 657 heterophylla Pursh

(P. circinata A. Gray, not Jacq.)

I $65^{8}$ heterosperma Parish

I 659 hirsuta Nutt.

I 660 hispida A. Gray

I 66 I brachyantha Coville

I 662 humilis T. \& G.

II663 calycosa A. Gray

I 664 hydrophylloides Torr.

I 665 Idahoensis Henderson

I 666 imbricata. Greene

I 667 inconspicua Greene

I 668 infundibuliformis Torr.

I 669 integrifolia Torr.

I 670 Palmeri A. Gray

I 67 I intermedia Wooton

I 672 Ivesiana Torr.

ri673 laxa Small

I 674 Lemmoni A. Gray

Ii675 leptostachya Greene

I 676 leucophylla Torr.

Ir677 leticantha Lemmon

I 678 linearis (Pursh) Holzinger (P. Menziesii Torr.)

I 679 loasaefolia 'Torr.

I 1680 longipes 'Torr.

I 681 Lyallii (A. Gray) Rydb.

I 682 Lyoni A. Gray

I 683 malvaefolia Cham.

II684 minutissima Henderson

I 685 Mohavensis A. Gray

II686 exilis A. Gray

I 687 mutabilis Greene

I 688 nemoralis Greene

I 689 Neo-Mexicana Thurber

I 690 pachyphylla A. Gray

I 169 I Parishii A. Gray

I 692 Parryi Torr.

I 693 patuliflora A. Gray

II694 pauciflora Wats.

I 695 pedicellata A. Gray

I 696 perityloides Coville

I 1697 pinetorum Jones

I 698 platyloba A. Gray

I 699 Popei T. \& G. 

I 700 Pringlei A. Gray

I 7 O procera A. Gray

I 702 pulchella A. Gray

I 703 Purpusi T. S. Brandegee

I 704 Purshii Buckley

I 705 pusilla Torr.

I 706 ramosissima Dougl.

I 707 suffrutescens Parry

II 708 Rattani A. Gray

I 709 rotundifolia Torr.

I 7 IO rugulosa Lemmon

I III saxicola A. Gray

II 7 I 2 scabrella Greene

II 7 I 3 sericea (Graham) A. Gray

I I I 4 splendens Eastwood

I I I 5 strictiflora A. Gray

I 7 I 6 suaveolens Greene

II 717 tanacetifolia Benth.

I I I 8 thermalis Greene

II 79 umbrosa Greene

I 720 verna Howell

II 721 virgata Greene

II 722 ampliata Greene

II 23 Bernardina Greene

I 724 viscida Torr.

I 725 albiflora A. Gray

I 726 Whitlavia A. Gray

\section{Emmenanthe}

I 727 foliosa Jones

I 728 glaberrima Torr.

I 729 glandulifera Torr.

II730 lutea A. Gray

II73I parviflora A. Gray

I $73^{2}$ penduliflora Benth.

II 733 pusilla A. Gray

I 734 salina Aven Nelson

II 735 scopulina Aven Nelson

\section{Conanthus}

(Nama L. I759, not L. I753) I 736 angustifolius (A.Gray) Heller II 737 aretioides (H. \& A.) Wats. I $73^{8}$ carnosus Wooton II 739 Coulteri (A. Gray) Heller I 740 demissus (A. Gray) Heller II 74I densus Lemmon I 742 depressus Lemmon I 743 Havardi (A. Gray) Heller
I 744 hispidus (A. Gray) Heller

I 745 Jamaicensis (L.) Heller

I 746 origanifolium (H. B. K.)

Heller

I 747 pusillus Lemmon

I 748 stenocarpus (A. Gray) Heller

I 749 stenophyllus (A. Gray) Heller

\section{Tricardia}

I $75^{\circ}$ Watsoni Torr.

\section{Capnorea}

(Hesperochiron)

I 75 I ciliata Greene

I 752 nana (Lindl.) Raf.

(H. Californicus Wats.)

I 753 pumila (Dougl.) Greene

\section{Romanzoffia}

I 754 Sitchensis Bong.

I 755 Unalaschkensis Cham.

\section{Eriodictyon}

I 756 angustifolium Nutt.

I 757 pubens A. Gray

I $75^{8}$ Californicum (H. \& A.) Torr. (E. glutinosum Benth.)

I 759 crassifolium Benth.

I 760 niveum Eastwood

I 76I Parryi (A. Gray) Greene (Nama Parryi A. Gray)

I 762 Rothrockii (A. Gray) Greene (Nama Rothrockii A. Gray)

I 763 tomentosum Benth.

I 764 Traskiae Eastwood

\section{Lemmonia}

Ir765 Californica A. Gray

\section{Nama}

\section{(Hydrolea)}

I 766 affinis (A. Gray) Kuntze

I 767 corymbosa (Ell.) Kuntze

I 768 ovata (Nutt.) Britton

I 769 quadrivalvis (Walt.) Kuntze (H. Caroliniana Michx.)

\section{BORAGINACEAE}

\section{Cordia}

I 770 Boissieri A. DC. 

II 77 I globosa H. B. K.

II 772 podocephala Torr.

I 773 Sebestena L.

\section{Bourreria}

11774 Havanensis (R. \& S.) Miers I 775 radula (Poir.) Don

\section{Ehretia}

I 1776 elliptica DC.

\section{Coldenia}

II 777 brevicalyx Wats.

II 778 canescens DC.

I1779 Greggii A. Gray

I 780 hispidissima A. Gray

I 78I Nuttallii Hook.

II 782 Palmeri A. Gray

\section{Tournefortia}

II 783 gnaphalodes R. Br.

I 784 mollis A. Gray

I 785 volubilis L.

\section{Cochranea}

I 786 anchusaefolia (Poir.) Guerke (Heliotropium anchusaefolium Poir.)

\section{Heliotropium}

I 787 angustifolium Torr. I 788 confertifolium Torr. I 789 convolvulaceum (Nutt.)

I 790 Curassavicum L.

I I79I Europaeum L.

I 792 glabriusculum A. Gray

I 793 Greggii Torr.

I 794 Indicum L.

I 795 inundatum Sw.

II 796 parviflorum $\mathrm{Sw}$.

I 797 phyllostachyum Torr.

I 798 polyphyllum Lehm.

II 799 Leavenworthii A. Gray I 800 tenellum (Nutt.) Torr.

\section{Pectocarya}

$1180 \mathrm{I}$ linearis DC.

I 802 penicillata H. \& A.

I 803 pusilla A. Gray

II 804 setosa A. Gray

\section{Eritrichium}

(Omphalodes)

I 805 aretioides DC.

I 806 elongatum Rydb.

I 1807 Chamissonis DC.

I 808 Howardi (A. Gray) Rydb.

\section{Cynoglossum}

I 809 grande Dougl.

II8Io laeve A. Gray

I 8 I I Howardi A. Gray

I 8 I 2 occidentale A. Gray

I 8 I 3 officinale $\mathrm{L}$.

II8I4 Virginicum L.

\section{Lappula}

\section{(Echinospermum)}

II8I5 Americana (A. Gray) Rydb.

I 8 I6 ciliata (Dougl,) Greene

II 8 I 7 coerulescens Rydb.

II 8 I 8 collina Greene

II8I9 coronata Greene

I 820 deflexa (Wahl.) Greene

I 82 I desertorum Greene

I 822 foliosa Aven Nelson

I 823 diffusa (Lehm.) Greene

I 824 erecta Aven Nelson

I 825 floribunda (Lelım.) Greene

I 826 Fremontii (Torr.) Greene

II 827 heterosperma Greene

I 828 hispida (A. Gray) Greene

A. Gray Ir829 Lappula (L.) Karst.

II 830 leptophylla Rydb.

I 83 I montana Greene

I 1832 nervosa (Kellogg) Greente

(E. Californicum A. Gray)

I 833 occidentalis (Wats.) Greene

I 834 pinetorum Greene

I 835 Texana (Scheele) Britton

I 836 ursina Greene

I 837 Virginiana (I..) Greene

\section{Allocarya}

(Krynitzkia in part)

I 838 Austinae Greene

I 839 Californica (F. \& M.) Greene

I 840 Chorisiana (C. \& S.) Greene

I 84 I Cooperi (A. Gray) Greene

I 842 Cusickii Greene 

I 843 diffusa Greene

I 844 echinoglochin Greene

I 845 Greenei (A. Gray) Greene

(Echinospermum Greenci A. Gray)

I 846 Hendersoni Aven Nelson

I 847 Hickmani Greene

II 848 hirta Greene

II 849 hispidula Greene

I 850 humistrata Greene

I 85 I leptoclada Greene

I 852 lithocarya Greene

I 853 mollis (A. Gray) Greene

I 854 myriantha Greene

I 855 Nelsoni Greene

I 856 nitens Greene

I 857 penicillata Greene

I 858 plebeia (C. \& S.) Greene

I 859 salsa T. S. Brandegee

I 860 Scouleri (H. \& A.) Greene

II86I scopulorum Greene

$$
\text { (K. Californica A. Gray) }
$$

I 862 scripta Greene

I 863 stipitata Greene

I 864 stricta Greene

I 1865 tenera Greene

I 866 trachycarpa (A. Gray) Greene I 867 vestita Greene

\section{Eremocarya}

(Krynitzkia in part)

I 868 lepida (A. Gray) Greene I 869 micrantha (Torr.) Greene

\section{Piptocalyx}

(K'rynitzkia in part)

I 870 circumscissus (H. \& A.) Torr. I 87 I dichotomus Greene

\section{Sonnea}

(Plagiobothrys in part)

' ir 872 foliacea Greene

I 873 glomerata (A. Gray) Greene

I 874 Harknessii Greene

I 875 hispida (A. Gray) Greene

I 876 Jonesii (A. Gray) Greene

I 877 Kingii (Wats.) Greene

\section{Plagiobothrys}

I1878 Arizonicus Greene

I 879 Catalinensis A. Gray
I 880 asper Greene

I 188 I Californicus Greene

I 882 campestris Greene

I 883 canescens Benth.

I 884 apertus Greene

I 885 colorans Greene

I 886 Cooperi A. Gray

I 887 echinatus Greene

I 888 microcarpa Greene

I 889 nothofulvus A. Gray

I 890 parvulus Greene

I 89 I Pringlei Greene

(Echidocarya Arizonica A. Gray)

I 892 rufescens F. \& M.

I 893 Shastensis Greene

I 894 tenellus A. Gray

I 895 Torreyi A. Gray

I 896 ursinus A. Gray

\section{Oreocarya}

(Krynitzkia in part)

I 897 abortiva Greene

I 898 affinis Greene

I 899 perennis Aven Nelson

I 900 Bakeri Greene

I 90 I caespitosa Aven Nelson

I 1902 cinerea Greene

I I 903 confertiflora Greene

I 1904 flava Aven Nelson

I 905 flavoculata Aven Nelson

I 906 spatulata Aven Nelson

I 1907 fulvocanescens (A. Gray)

Greene

I I go8 glomerata (Pursh) Greene

I 909 holoptera (A. Gray) Greene

I I 9 Io humilis Greene

II9I I interrupta Greene

I I9I 2 lencophaea (Dougl.) Greene

I 1913 longiflora Aven Nelson

Ir 9I4 lutescens Greene

II9I 5 multicanlis (Torr.) Greene

I 1916 nubigena Greene

I I9I7 Palmeri (A. Gray) Greene

I 918 ramulosissima Aven Nelson

I 9 I 9 sericea (A. Gray) Greene

I 1920 setosissima (A. Gray) Greene

I 92 I suffruticosa (Torr.) Greene

( $k$. Jamesii A. Gray) 

I 922 thyrsiflora Greene

I 923 virgata (Porter) Greene

\section{Cryptanthe}

(Krynitzkia in part)

I 924 affinis (A. Gray) Greene

I 925 ambigua (A. Gray) Greene

I 926 angustifolia (Torr.) Greene

I 927 barbigera (A. Gray) Greene

Ir 928 calycosa (A. Gray) Rydb.

I 929 Cedrosensis Greene

I 1930 Clevelandi Greene

I I 93 I costata T. S. Brandegee

I 932 crassisepala (T. \& G.) Greene

I 933 crinita Greene

I 934 cylloptera Greene

I 935 denticulata Greene

I 1936 dumetorum Greene

I 1937 echinella Greene

I 938 excavata T. S. Brandegee

I 939 Fendleri (A. Gray) Greene

I 940 flaccida (Lehm.) Greene

(K. oxycarya A. Gray)

I I 94 I foliosa Greene

I 942 geminata Greene

I 943 glomeriflora Greene

I 944 hispidissima Greene

I 945 intermedia (A. Gray) Greene

I 946 Jonesii (A. Gray) Greene

I 947 Kelseyana Greene

I 948 leiocarpa (F. \& M.) Greene

I 949 maritima Greene

I 950 micromeres (A. Gray) Greene

I 95 I microstachys Greene

I 1952 Mohavensis Greene

I 1953 muriculata (A. DC.) Greene

I 954 montana Aven Nelson

II955 nemaclada Greene

I 1956 oxygona (A. Gray) Greene

I 1957 Pattersoni (A. Gray) Greene II958 patula Greene

I 959 polycarpa Greene

I 960 Pondii Greene

I I96I pterocarpa (Tort.) Greene

I 962 pusilla ('T. \& G.) Greene

I 963 racemosa (A. Gray) Greene

II 964 ramosa (A. DC.) Greene

I 965 ramosissima Greene
I 966 Rattani Greene

II967 recurvata Coville

I 968 rostellata Greene

I 969 sparsiflora Greene

I 970 submollis (A. Gray) Coville (K. Utahensis A. Gray)

I 197 I Texana (A. DC.) Greene

II972 Torreyi (A. Gray) Rydb. (K. Torreyana A. Gray)

I 973 Watsonii (A. Gray) Greene

\section{Amsinckia}

I 974 carnosa Jones

I 975 echinata A. Gray

I 976 intermedia F. \& M.

I 977 lycopsoides Lehm.

I 978 bracteosa A. Gray

II979 maritima Eastwood

I I980 spectabilis F. \& M.

II98I St. Nicholai Eastwood

I 982 tesselata A. Gray

I 983 vernicosa $H$. \& $A$.

II984 grandiflora A. Gray

\section{Asperugo}

I 985 procumbens L.

\section{Symphytum}

I 986 officinale L.

\section{Borago}

I 987 officinalis L.

\section{Lycopsis}

I 988 arvensis L.

\section{Myosotis}

I 989 alpestris Schmidt

I 990 arvensis (L.) Lam.

I I99I caespitosa Schulz

I 992 alpestris Koch

I 993 collina Hoffm.

I 994 laxa Lehm.

I 995 macrosperma Engelm.

I 996 palustris (L.) Lam.

I 1997 sylvatica Hoffm.

I 1998 versicolor (Pers.) Reichenb.

I 999 Virginica (L.) B. S. P.

(M. verna Nutt.) 



\section{Pneumaria}

I 2000 maritima (L. $)$ Hill (Mertensia maritima Don)

\section{Mertensia}

I 200 I alpina (Torr.) Don

I 2002 Arizonica Greene

I 2003 Bakeri Greene

I 2004 brachyloba Greene

I 2005 ciliata (Torr.) Don

I 2006 Fendleri A. Gray

I 2007 foliosa Aven Nelson

I 2008 Franciscana Heller

I 2009 fusiformis Greene

I2010 intermedia Rydb.

I 20 I I lanceolata (Pursh) DC.

I 2012 linearis Greene

I20I3 longiflora Greene

I2014 MacDongalii Heller

I 2015 nivalis (Wats.) Rydb.

I20I6 oblongifolia (Nutt.) Don

I 2017 paniculata (Ait.) Don

I 2018 papillosa Greene

I 2019 platyphylla Heller

I 2020 pilosa (Cham.) Don

I 2021 polyphylla Greene

I 2022 pratensis Heller

I 2023 punctata Greene

I 2024 Sibirica (L.) Don

I 2025 Drummondii A. Gray

I 2026 stomatechoides Kellogg

I 2027 strigosa Greene

I 2028 subcordata Greene

I 2029 tubiflora Rydb.

I 2030 Tweedyi Rydb.

I 2031 Virginica (L.) DC.

I 2032 viridis Aven Nelson

\section{Lithospermum}

I 2033 albicans Greene I 2034 arvense L.

I 2035 asperum Aven Nelson

I 2036 Californicum A. Gray

I 2037 canescens (Michx.) Lehm.

I 2038 ciliolatum Greene

I 2039 Cobrense Greene

I 2040 glabrum A. Gray

I204I Gmelini (Michx.)A.S.Hitchc.

(L. hirtum Lehm.)
I2042 lanceolatum Rydb.

I 2043 latifolium Michx.

I 2044 laxum Greene

I 2045 linearifolium Goldie (L. angustifolium Michx.)

I 2046 Matamorense DC.

I 2047 multiflorum Torr.

I 2048 oblongum Greene

I 2049 officinale L.

I 2050 pilosum Nutt.

I 205I Torreyi Nutt.

I 2052 tuberosum Rugel

I 2053 viride Greene

\section{Onosmodium}

I 2054 Bejariense DC.

I 2055 Carolinianum (Lam.) A. DC.

I 2056 molle Michx.

I 2057 Thurberi A. Gray

I 2058 Virginianum (L.) A. DC.

\section{Echium}

I 2059 vulgare L.

\section{Harpagonella}

I 2060 Palmeri A. Gray

\section{VERBENACEAE \\ Verbena}

I206I angustifolia Michx.

I 2062 bipinnatifida Nutt.

I 2063 Bonariensis L.

I 2064 bracteosa Michx.

I 2065 brevibracteata A. Gray

I 2066 Canadensis (L.) Britton

( $V$. Aubletia Jacq.)

I 2067 canescens H. B. K.

I 2068 Neo-Mexicana A. Gray

I 2069 Caroliniana Michx.

I 2070 ciliata Benth.

I $207 \mathrm{I}$ confinis Greene

I 2072 Halei Small

I 2073 Hanseni Greene

12074 hastata L.

I 2075 MacDougalii Heller

$\mathrm{r} 2076$ officinalis $\mathrm{L}$.

I 2077 perennis Wooton

I 2078 pinnatifida Lam.

I 2079 polystachya H. B. K.

I 2080 prostrata $\mathrm{R}$. Br. 

I 208 I quadrangulata Heller

I 2082 remota Benth.

12083 riparia Raf.

I2084 robusta Greene

I 2085 rudis Greene

I 2086 stricta Vent.

I 2087 Tampensis Nash

I 2088 urticaefolia L.

I 2089 venosa Gillies \& Hook.

I 2090 Wrightii A. Gray

I2091 xutha Lehm.

\section{Lantana}

I 2092 Camara L.

I 2093 canescens H. B. K.

I 2094 involucrata L.

I 2095 macropoda Torr.

\section{Lippia}

I 2096 graveolens H. B. K. I 2097 lantanoides (L.) Coult. (L. geminata H. B. K.)

I 2098 ligustrina (Lag.) Britton (L. lycioides Steud.)

I 2099 Wrightii A. Gray

\section{Phyla}

(Lippia in part)

I 2 IOO cuneifolia (Torr.) Greene

r2Ior lanceolata (Michx.) Greene I 2102 nodiflora (L.) Greene

\section{Bouchea}

I2103 Ehrenbergii Cham. I 2 IO4 linifolia A. Gray I 2 IO5 spathulata Torr.

\section{Stachytarpheta}

I 2 Io6 Jamaicensis (L.) Vahl

\section{Priva}

12107 echinata Juss.

\section{Citharexylum}

I 2108 brachyanthum A. Gray

I 2 IO9 villosum Jacq.

\section{Duranta}

I 2 r ro Plummeri Jacq.

\section{Callicarpa}

I2III Americana L.

\section{Vitex}

I 2 I 2 Agnus-castus L.

Avicennia

I 2 I 3 nitida Jacq.

\section{LABIATAE}

\section{Ajuga}

I 2 II4 reptans L.

\section{Teucrium}

I 2 II 5 Canadense L.

I 2 I 6 angustatum A. Gray

I 2 I 7 Cubense L.

I 2 I 8 depressum Small

I 2 I 9 laciniatum Torr.

I2I2O Nashii Kearney

I2I2I occidentale A. Gray

\section{Tetraclea}

I 2122 Coulteri A. Gray

\section{Isanthus}

I 2 I 23 brachiatus (L.) B. S. P. (I. coeruleus Michx.)

\section{Trichostema}

I 2124 Arizonicum A. Gray

I 2 I 25 dichotomum L.

I 2 I 26 lanatum Benth.

I2I27 denudatum A. Gray

I 2 I 28 lanceolatum Benth.

I2I 29 laxum A. Gray

I 2 30 lineare Nutt.

I 2 I 3 micranthum A. Gray

I 2132 oblongum Benth.

I 2 I 33 suffrutescens Kearney

I 2 I 34 ovatum Curran

\section{Salizaria}

I2I35 Mexicana Torr.

\section{Scutellaria}

I 2 36 Altamaha Small

I2I 37 angustifolia Pursh

I2I 38 arenicola Small

I2I39 antirrhinoides Benth.

I2 I40 Bolanderi A. Gray

I2I4I brevifolia A. Gray

I 2 I 42 Brittoni Porter

(S. resinosa A. Gray, not Torr.) 

I 2 43 Californica A. Gray

I 2 I 44 campestris Britton

(S. parvula var. mollis A. Gray)

I 2 I 45 cardiophylla.Engelm.\& Gray

I2I46 cordifolia Muhl.

I2I47 Drummondii Benth.

I 2 I 48 Floridana Chapm.

I2149 Footeana Mulford

I 2 I 50 galericulata L.

I2I 5I incana Muhl.

I2I52 punctata (Chapm.) Mohr

I 2 I53 integrifolia I.

I2I54 major Chapm.

I 2 I 55 multiglandulosa Kearney

I 2 I 56 lateriflora L.

I2I57 montana Chapm.

I2 I58 nana A. Gray

I 2 I 59 nervosa Pursh

I2160 Ocmulgee Small

I2I6I parvula Michx.

I 2 I62 pilosa Michx.

I2I63 hirsuta (Short) A. Gray

I 2 I64 resinosa Torr.

(S. Wrightii A. Gray)

I2I65 saxatilis Riddell

I2I66 arguta A. Gray

I2167 serrata Andr.

I 2 I68 syphocampyloides Vatke

(S. angustifolia var. canescens)

I 2 I69 tuberosa Benth.

I 2170 venosa Kearney

I 2 I I virgulata Aven Nelson

\section{Marrubium}

I 2 I 72 vulgare L.

\section{Agastache}

\section{(Lophanthus; Vleckia)}

I 2 I 73 anethiodora (Nutt.) Britton

(L. anisatus Benth.)

I 2 I 74 nepetoides (L.) Kuntze

I 2 I 75 occidentalis (Piper) Heller

I 2 I 6 scrophulariaefolia (Willd.)

Kuntze

I 2 I 77 urticifolia (Benth.) Rydb.

\section{Meehania}

I 2 I 78 cordata (Nutt.) Britton

(Cedronella cordata Benth.) I2206 tuberosa L.

\section{Brittonastrum}

\section{(Cedronella)}

12 I79 canum (Hook.) Briquet

I 2 r80 lanceolatum (A. Gray) Heller

I 2I 8I Mexicanum (Kunth) Briquet

I 2 I 82 micranthum (A.Gray)Briquet

I 2 I 83 pallidiflorum Heller

I 2 184 pallidum (Lindl.) Briquet

I 2 I 85 rupestre (Greene) Heller

\section{Nepeta}

I 2 I 86 Cataria L.

\section{Glecoma}

I 2 I 87 hederacea L.

(Nepeta Glechoma Benth.)

\section{Dracocephalum}

I 2 I 88 Moldavica L.

I 2189 parviflorum Nutt.

\section{Prunella}

I 2 Igo laciniata L.

I 2191 vulgaris L.

I 2192 scabrella Pollard

\section{Brazoria}

I 2 I93 scutellarioides

Engelm. \& Gray

I2I94 truncata Engelm. \& Gray

\section{Physostegia}

I 2 I95 denticulata (Ait.) Britton

1 2196 digitalis Small

I 2197 intermedia (Nutt.) A. Gray

I 2I98 leptophylla Small

I 2I99 parviflora Nutt.

I 2200 Virginiana (L.) Benth.

I220I obovata (Ell.) A. Gray

\section{Synandra}

I 2202 hispidula (Michx.) Britton

(S. grandiflora Nutt.)

\section{Macbridea}

I 2203 alba Chapm.

I 2204 pulchra Eill.

\section{Leonotis}

I 2205 nepetaefolia R. Br.

\section{Phlomis}





\section{Galeopsis}

I 2207 Ladanum L.

I 2208 Tetrahit L.

\section{Lamium}

I 2209 album L.

I22IO amplexicaule L.

122 I I intermedium Fries

I 2212 maculatum L.

122 I 3 purpureum L.

\section{Leonurus}

I2214 Cardiaca L.

12215 marrubiastrum L.

I22I6 Sibiricus L.

\section{Ballota}

I22I 7 nigra L.

\section{Stachys}

I 2218 acuminata Greene

I2219 agraria C. \& S.

I 2220 ajugoides Benth.

I222I albens A. Gray

12222 ambigua (A. Gray) Britton

12223 arvensis $\mathrm{L}$.

I2224 aspera Michx.

I 2225 tenuiflora A. S. Hitchc.

I 2226 Betonica Benth.

12227 Bigelovii A. Gray

I 2228 bracteata Greene

12229 bullata Benth.

I 2230 Chamissonis Benth.

I223I ciliata Dougl.

I 2232 coccinea Jacq.

I 2233 Coolyae Heller

12234 cordata Riddell

12235 Drummondii Benth.

12236 Emersoni Piper

12237 Floridana Shuttlw.

$1223^{8}$ Germanica L.

I2239 hyssopifolia Michx.

I 2240 ingrata Greene

I224I lanuginosa Greene

I 2242 littoralis Greene

12243 malacophylla Greene

I 2244 palustris L.

12245 pubens (A. Gray) Heller

12246 pycnantha Benth.

I2247 Rothrockii A. Gray
I 2248 scopulorum Greene

I 2249 stricta Greene

12250 tenuifolia Willd.

(S. aspera var. glabra A. Gray)

I225I velutina Greene

\section{Betonica}

12252 officinalis L.

\section{Acanthomintha}

I 2253 ilicifolia A. Gray

I 2254 lanceolata Curran

\section{Salvia}

I 2255 albiflora Mart. \& Gal.

12256 Pringlei A. Gray

12257 angustifolia Cav.

$1225^{8}$ glabra A. Gray

I 2259 Arizonica A. Gray

I 2260 azurea Lam.

I 226I ballotaeflora Benth.

I 2262 Blodgettii Chapm.

12263 carduacea Benth.

I 2264 carnosa Dougl.

I 2265 Chapmani A. Gray

I 2266 coccinea $\mathrm{L}$.

I2267 Columbariae Benth.

I 2268 farinacea Benth.

I2269 Greggii A. Gray

I 2270 Henryi A. Gray

I227I lanceolata Willd.

12272 Lemmoni A. Gray

12273 lyrata L.

I 2274 occidentalis Sw.

12275 Parryi A. Gray

I 2276 pentstemonoides Kunth

I 2277 Pitcheri Torr.

(S. azurea var. grandiflora Benth.)

I 2278 pratensis L.

I2279 privoides Benth.

I 2280 Garberi Chapm.

I 228 I ramosissima Fernald

(S. chamaedryoides A.Gray, not Cav.)

I 2282 Regia Cav.

12283 Roemeriana Scheele

12284 Sclarea L.

I 2285 serotina L.

I 2286 spicata R. \& S.

I 2287 subincisa Benth. 

I 2288 urticifolia L.

$\$ 2289$ verbenacea L.

\section{Salviastrum}

(Salvia in part)

12290 canescens (A. Gray) Briquet

I229I Engelmanni (A.Gray)Briquet I 2292 Texana Scheele

\section{Ramona}

(Audibertia)

I 2293 capitata (A. Gray) Briquet

12294 Clevelandi (A. Gray) Briquet I 2295 Dorrii (Kellogg) Briquet I 2296 grandiflora (Benth.) Briquet I 2297 humilis (Benth.) Greene I 2298 nivea (Benth.) Briquet 12299 Palmeri (A. Gray) Briquet I2300 polystachya (Benth.) Greene I230I stachyoides (Benth.) Briquet

\section{Monarda}

I2302 Bradburiana Beck

I 2303 citriodora Cerv.

I2304 Clinopodia L.

I 2305 clinopodioides A. Gray

I 2306 didyma L.

I 2307 fistulosa L.

12308 media Willd.

I2309 pectinata Nutt.

I 23 Io punctata L.

I23II lasiodonta A. Gray

I23I2 leucantha Nash

I23I3 Russeliana Nutt.

I23I4 scabra Beck

I23I5 stricta Wooton

\section{Blephilia}

I23I6 ciliata (L.) Raf.

I 23 I 7 hirsuta (Pursh) Torr.

I2318 glabrata Fernald

\section{Sphacele}

I 2319 calycina Benth.

12320 glabella A. Gray

I232I Wallacei A. Gray

I2322 fragrans Greene

\section{Hedeoma}

I2323 acinoides Scheele I2324 blepharodonta Greene
I 2325 ciliata Benth.

I 2326 costata A. Gray

I 2327 dentata Torr.

12328 diffusa Greene

I 2329 Drummondii Benth.

12330 graveolens Chapm.

I 233 I hispida Pursh

I 2332 hyssopifolia A. Gray

I 2333 nana (Torr.) Greene (H. thymoides A. Gray)

I 2334 oblongifolia (A. Gray) Heller

I 2335 plicata Torr.

I 2336 pulegioides (L.) Pers.

I 2337 Reverchoni A. Gray

I 2338 sancta Small

I 2339 serpyllifolia Small

\section{Poliomintha}

I2340 glabrescens A. Gray

I 234I incana A. Gray

12342 mollis A. Gray

\section{Melissa}

I 2343 officinalis L.

\section{Satureia}

I 2344 hortensis L.

I 2345 rigida Bartr.

\section{Micromeria}

I2346 Brownei Benth.

12347 pilosiuscula A. Gray

r2348 Chamissonis (Benth.) Greene (M. Douglasii Benth.)

I 2349 purpurea (Kellogg) A. Gray

\section{Clinopodium}

(Calamintha)

I2350 Acinos (I.0) Kuntze

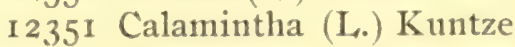

(C. officinalis Moench)

I 2352 Carolinianum (Michx.) Heller

I 2353 coccineum (Benth.) Kuntze

12354 dentatum (Chapm.) Kuntze

I 2355 glabellum (Michx.) Kuntze

I 2356 glabrum (Nutt.) Kuntze

(C. Nuttallii Benth.)

I 2357 mimuloides (Benth.) Kuntze

I2358 Nepeta (L.) Kuntze

12359 vulgare $\mathrm{L}$.

(C. Clinopodium Benth.) 



\section{Conradina}

12360 canescens A. Gray

I 236 I puberula Small

\section{Pogogyne}

I 2362 Douglasii Benth.

I 2363 nudiuscula A. Gray

I 2364 parviflora Benth.

\section{Hediomoides}

(Pogogyne in part)

I 2365 serpylloides (Torr.) Briquet I 2366 ziziphoroides (Benth.)Briquet

\section{Ceranthera}

I 2367 densiflora (Benth.) A. Gray I 2368 linearifolia E11.

\section{Hyssopus}

I 2369 officinalis L.

\section{Origanum}

I 2370 vulgare $\mathrm{L}$.

\section{Monardella}

1237 I Breweri A. Gray

I 2372 candicans Benth.

12373 exilis A. Gray

I 2374 discolor Greene

I2375 Donglasii Benth.

I2376 hypoleuca A. Gray

I 2377 lanceolata A. Gray

I 2378 microcephala A. Gray

I 2379 linoides A. Gray

I 2380 stricta S. B. Parish

I 238 I leucocephala A. Gray

12382 macrantha A. Gray

12383 nana A. Gray

I 2384 odoratissima Benth.

I 2385 Palmeri A. Gray

I 2386 Pringlei A. Gray

12387 tenuiflora Wats.

I 2388 undulata Benth.

I 2389 villosa Benth.

I 2390 glabella A. Gray

I 239 I leptosiphon Torr.

\section{Koellia}

\section{(Pycnanthemum)}

I 2392 albescens (T. \& G.) Kuntze I 2393 aristata (Michx.) Kuntze
12394 Beadlei Small

r 2395 Californica (Torr.) Kuntze

I 2396 clinopodioides (T. \& G.)

Kuntze

I 2397 flexuosa (Walt.) MacM. (P. linifolium Pursh)

12398 hyssopifolia (Benth.) Britton

I 2399 incana (L.) Kuntze

I 2400 leptodon (A. Gray) Kuntze

I 240 I montana (Michx.) Kuntze

I 2402 mutica (Michx.) Britton

I 2403 nuda (Nutt.) Kuntze

I 2404 pilosa (Nutt.) Britton

I 2405 pycnanthemoides (Leavenw.)

(P. Tullia Benth.) Kuntze

I 2406 Torreyi (Benth.) Kuntze

I 2407 verticillata (Michx.) Kuntze

I 2408 Virginiana (L.) MacM. (P. lanceolatum Pursh)

\section{Thymus}

I 2409 serpyllum L.

\section{Cunila}

I2410 Mariana L.

\section{Lycopus}

I24II Americanus Muhl.

(L. simuatus Ell.

I 24I 2 asper Greene

I 2413 Europaeus L.

I 24I 4 lacerus Greene

I 24 I 5 lucidus Turcz.

I 2416 maritimus Greene

I 2417 rubellus Moench

I 24 I 8 sessilifolius A. Gray

I 2419 pubens A. Gray

I 2420 Virginicus L.

\section{Mentha}

I 242 I alopecuroides Hull

I 2422 aquatica $\mathrm{L}$.

I 2423 arvensis L.

I 2424 Canadensis L.

I 2425 glabrata Benth.

I 2426 citrata Ehrh.

I 2427 crispa L.

I 2428 gentilis L.

I 2429 longifolia (L.) Huds.

(M. sylaestris L.) 

I 2430 piperita $\mathrm{L}$.

I 243 I Pulegium L.

I 2432 rotundifolia (L.) Huds.

I 2433 rubella Rydb.

I 2434 sativa L.

I 2435 spicata L.

(M. viridis L.)

\section{Collinsomia}

12436 Canadensis L.

12437 punctata A. Gray

$1243^{8}$ scabriuscula Ait.

\section{Micheliella}

(Collinsonia in part)

I2439 anisata (Sims) Briquet

I 2440 verticillata (Baldwin) Briquet

\section{Perilla}

I 244I frutescens ( $\mathrm{L}_{i}$ Britton

( $P$. ocimoides $\mathrm{L}$.)

I 2442 Nankinensis(Lour.)Britton

\section{Elscholtzia}

I 2443 Patrinii (Lepech.) Kuntze (E. cristata Willd.)

\section{Mesosphaerum}

(Hyptis)

I2444 Emoryi ('Torr.) Kuntze

I 2445 pectinatum (L.) Kuntze

I2446 rugosum (L.) Pollard (H. spicata Poit.)

I 2447 spicigerum (Lam.) Kuntze

\section{Ocimum}

12448 micranthum Willd.

\section{Molucella}

I 2449 laevis L.

SOLANACEAE

\section{Physalodes}

(Nicandra)

I2450 Physalodes (L.) Britton

\section{Lycium}

1245I Andersoni A. Gray

I $245^{2}$ Wrightii A. Gray

I 2453 Berlandieri Dunal

12454 Californicum Nutt.

12455 Arizonicum A. Gray
I 2456 Carolinianum Walt.

I 2457 Cedrosense Greene

$1245^{8}$ Cooperi A. Gray

I 2459 pubiflora A. Gray

I 2460 Fremontii A. Gray

I 246I Bigelovii A. Gray

I 2462 gracilipes A. Gray

12463 Hassei Greene

I 2464 macrodon A. Gray

I 2465 pallidum Miers.

I 2466 Parishii A. Gray

12467 parviflorum A. Gray

I 2468 puberulum A. Gray

I 2469 Richii A. Gray

12470 Shockleyi A. Gray

I 247 I Torreyi A. Gray

I 2472 filiforme Jones

I 2473 verrucosum Eastwood

I 2474 vulgare (Ait. f.) Dunal

\section{Oryetes}

I 2475 Nevadensis Wats.

\section{Margaranthus}

I 2476 Lemmoni A. Gray

I 2477 purpurascens Rydb.

I 2478 solanaceus Schlecht.

\section{Hyoseyamus}

I 2479 niger L.

\section{Chamaesaracha}

I 2480 conoides (Moricand) Britton (C. sordida A. Gray)

I 248 I Coronopus (Dunal) A. Gray

I 2482 crenata Rydb.

I 2483 nana A. Gray

\section{Physalis}

I 2484 Alkekengi L。

I 2485 ambigua (A. Gray) Britton

I 2486 angulata I.

12487 angustifolia Nutt.

I 2488 arenicola Kearney

I 2489 Barbadensis Jacq.

I 2490 Carpenteri Riddell

I 249 I ciliosa Rydb.

I 2492 comata Rydb.

I 2493 crassifolia Benth.

I 2494 cardiophylla ('Torr.)

A. Gray 

I2495 Elliottii Kunze

I 2496 Fendleri A. Gray

I 2497 cordifolia A. Gray

I 2498 fuscomaculata Du Rouv.

I 2499 Greenei Vasey \& Rose

I 2500 hastata Rydb.

I 250 I hederaefolia A. Gray

(P. Palmeri A. Gray)

I2502 puberula A. Gray

12503 heterophylla Nees

I2504 ixocarpa Brot.

(P. aequata Jacq.)

I 2505 Lagascae R. \& S.

(P. minima Roxb. not L.)

I2506 lanceolata Michx.

12507 lancifolia Nees

I 2508 longifolia Nutt.

(P. lanceolata var. laevigata A. Gray)

I2509 macrophysa Rydb.

I 25 IO mollis Nutt.

I25II cinerascens (Dunal)

A. Grav

I25I 2 parvifolia Rydb.

I25I3 monticola Mohr.

I25I 4 muricatula Greene

I2515 Neo-Mexicana Rydb.

I 25I6 nyctaginea Dunal

I 25I7 obscura Michx.

I 2518 Peruviana L.

I 519 latifolia (Lam.) Dumal

I 2520 Philadelphica Lam.

I 252 I polyphylla Greene

I 2522 pruinosa L.

I 2523 pubescens L.

I 2524 pumila Nutt.

(P. lanceolata var. hirta A. Gray)

I 2525 rigida Pollard \& Ball

I 2526 rotundata Rydb.

I 2527 Texana Rydb.

I 2528 versicolor $R y d b$.

I2529 Virginiana Mill.

I2530 intermedia Rydb.

I 253 I viscosa $\mathrm{L}$.

I 2532 maritima (Curtis) Rydb.

I 2533 Wrightii A. Gray

\section{Leucophysalis}

I 2534 grandiflora (Hook.) Rydb.

\section{Quincula}

I 2535 lobata ('Torr.) Raf. (Physalis lobata Torr.)

\section{Saracha}

(Bellinia)

I 2536 umbellata (Roth.) Don

\section{Capsicum}

I 2537 baccatum L.

I $253^{8}$ frutescens I.

\section{Solanum}

I 2539 aculeatissimum Jacq.

I 2540 Bahamense L.

I 254I Blodgettii Chapm.

I 2542 Carolinense L.

I 2543 cupuliferum Greene

I 2544 Douglasii Dunal

I 2545 Dulcamara L.

I2546 elaeagnifolium Cav.

I 2547 glaucum Dunal

I 2548 gracile Link

I 2549 heterodoxum Dunal

I $255^{\circ}$ hirsutum (Vahl) Dunal

I255I Jamesii Torr.

I 2552 nigrum L.

I 2553 Pseudo-Capsicum $I_{4}$.

I 2554 rostratum Dunal

I 2555 sisymbriifolium Lam.

I 2556 Torreyi A. Gray

I 2557 triflorum Nutt.

$1255^{8}$ triquetrum Cav.

I 2559 tuberosum boreale A. Citay

I 2560 umbelliferum Esch.

I256I verbascifolium $\mathrm{L}$.

I 2562 Xanti A. Gray

I 2563 Wallacei A. Gray

\section{Lycopersicon}

I 2564 Lycopersicon (L.) Karst. (L. esculentum Mill.)

\section{Salpichroa}

I 2565 rhomboidea Miers

I 2566 Wrightii A. Gray

\section{Datura}

I 2567 discolor Benth.

I 2568 inermis Jacq.

I 2569 Metel I. 

I 2570 meteloides DC.

1257 I Stramonium L. I 2572 Tatula L.

\section{Cestrum}

I2573 dinrnum L.

I 2574 Parqui L'Her.

\section{Nicotiana}

I2575 attenuata Torr.

I2576 Bigelovii Wats.

12577 Wallacei A. Gray

I 2578 Clevelandi A. Gray

I 2579 glauca Graham

12580 longiflora Cav.

12581 Palmeri A. Gray

I $25^{82}$ quadrivalvis Pursh

12583 multivalvis A. Gray

I2584 repanda Willd.

12585 rustica L.

I 2586 Tabacum undulata Sendt. 12587 trigonophylla Dunal

\section{Petumia}

I 2588 axillaris (Lam.) B. S. P.

(P. nyctaginiflora Juss.)

I $25^{89}$ parviflora Juss.

I 2590 violacea Lindl.

\section{Bouchetia}

1259 I erecta DC.

\section{Leptoglossis}

12592 Texana A. Gray

\section{SCROPHULARIACEAE}

\section{Verbascum}

I 2593 Blattaria L.

I 2594 Lychnitis L.

I 2595 phlomoides L.

I 2596 Thapsus L.

I 2597 virgatum With.

\section{Leucophyllum}

I 2598 minus A. Gray

12599 Texanum Benth.

\section{Cymbalaria}

I 2600 Cymbalaria (L.) Wettst.
Kickxia

(Linaria in part)

I 260 I Elatine (L.) Dumont.

I 2602 spuria (L.) Dumont

\section{Linaria}

12603 Canadensis (L.) Dumont

I 2604 Floridana Chapm.

I 2605 genistaefolia (L.) Mill.

I 2606 Linaria (L.) Karst.

(L. vulgaris Mill.)

12607 repens (L.) Mill.

\section{Mohavea}

I 2608 breviflora Coville I 2609 viscida A. Gray

\section{Antirrhinum}

I26ro Breweri A. Gray I 26 I Cooperi A. Gray

I 26 I 2 cornutum Bentl. I 26 I $_{3}$ Coulterianum Benth. I26I4 cyathiferum Benth. I 26 I 5 glandulosum Lindl. I26I6 Kingii Wats. I26I7 leptaleum A. Gray I 26 I 8 majus L.

I26I9 Nevinianum A. Gray I 2620 Nuttallianum Bentl. I 262 I effusum A. Gray I 2622 Orcuttianum A. Gray I 2623 Orontium I. I 2624 strictum L. I 2625 subcordatum A. Gray I 2626 subsessile A. Gray I 2627 vagans $A$. Gray I 2628 Bolanderi A. Gray I 2629 virga A. Gray

\section{Galvesia}

I 2630 speciosa A. Gray

\section{Maurandia}

I263I antirrhiniflora (Poir.) Willd. (Antirrhinum maurandioides Gray) I 2632 Wislizeni Engelm.

\section{Collinsia}

I 2633 arvensis Greene I 2634 bartsiaefolia Benth. (Linaria Cymbalaria Mill.) I 2635 bicolor Benth. 



I 2636 callosa S. B. Parish
I 637 Childii Parry
I2638 concolor Greene
I 2639 Corymbosa Herder
I 2640 Davidsonii Parish
I 64 I diversifolia Greene
I 2642 Franciscana Bioletti
I 2643 grandiflora Dougl.
I 2644 pusilla A. Gray
I 2645 Greenei A. Gray
I 2646 linearis A. Gray
I 647 Parryi A. Gray
I 2648 parviflora Dougl.
I 2649 Rattani A. Gray
I 2650 sparsiflora F. \& M.
I 265 I stricta Greene
I 2652 tinctoria Hartw.
I 2653 Torreyi A. Gray
I 2654 verna Nutt.
I 2655 violacea Nutt.
I 2656 Wrighti Wats.

\section{Tonella}

I 2657 floribunda A. Gray r 2658 tenella (Benth.) Heller ( $T$. collinsioides Nutt.)

\section{Serophularia}

I2659 Californica Cham. I2660 floribunda Greene 1266r coccinea A. Gray I 2662 leporella Bicknell I 2663 Marylandica L. r 2664 montana Wooton I 2665 occidentalis (Rydb.) Bicknell

\section{Chelone}

I 2666 glabra L. I 2667 Lyoni Pursh I 2668 nemorosa Doug1. I 2669 obliqua L.

\section{Pentstemon}

r.2670 acuminatus Dougl. I 267 I congestus Jones I 2672 albidus Nutt. r 2673 ambiguus Torr. I2674 Thurberi A. Gray I 2675 angustifolius Pursh (P. coeruleus Nutt.)
I 2676 antirrhinoides Benth.

I 2677 arenarius Greene

I 2678 arenicola Nelson I 2679 aridus Rydb.

I 2680 Arizonicus Heller 1268I attenuatus Dougl. I 2682 azureus Benth.

I 2683 angustissimus A. Gray

I 2684 Jaffreyanus A. Gray

I 2685 parvulus A. Gray

I 2686 baccharifolius Hook.

I 2687 barbatus (Cav.) Nutt.

I 2688 puberulus A. Gray

I 2689 trichander A. Gray

I 2690 Barrettae A. Gray

I 269I Brandegei Porter

I 2692 breviflorus Lindl.

I 2693 Bridgesii A. Gray

I 2694 caesius A. Gray

I 2695 caespitosus Nutt.

I 2696 - suffruticosus A. Gray

I 2697 calycosus Small

I 2698 canescens Britton

I 2699 centhranthifolius Benth.

I 2700 Clevelandi A. Gray

12701 Cobaea Nutt.

I 2702 collinus Aven Nelson

I 2703 Coloradoensis Aven Nelson

I 2704 confertus Dougl.

I 2705 aberrans Jones

I 2706 globosus Piper

I 2707 confusus Jones

I 2708 cordifolius Benth.

I 2709 corymbosus Benth.

I 27 IO Crandallii Aven Nelson

I 27 I I crassifolius Lindl.

(P. Menziesii var. Douglasii Gray)

I 2712 cristatus Nutt.

I 2 II 3 Cusickii A. Gray

I 27 I 4 cyananthus Hook.

I 27I 5 Davidsonii Greene

I 2716 deustus Dougl.

12717 pedicellatus Jones

I 2718 diffusus Dougl.

I 2719 Digitalis (Sweet) Nutt.

I 2720 dissectus Ell.

I 272 I Fastwoodiae Heller

I 2722 Eatoni A. Gray 

I 2723 undosus Jones

1 2724 ellipticus Coult. \& Fisher I 2725 floridus T. S. Brandegee I2726 Fremontii T. \& G. I 2727 subglaber A. Gray

12728 frutescens Lam.

I 2729 fruticiformis Coville

I 2730 fruticosus (Pursh) Greene

1273 I Gairdneri Hook.

I2732 hians Piper

I 2733 Oreganus A. Gray

I2734 geniculatus Greene

I 2735 glaber Pursh

I 2736 alpinus A. Gray

I 2737 glandulosus Lindl.

$1273^{8}$ glaucus Graham

12739 stenosepalus A. Gray

12740 gracilentus A. Gray

1274 I gracilis Nutt.

I 2742 grandiflorus Nutt.

I 2743 Guadalupensis Heller

I 2744 Hallii A. Gray

12745 Arizonicus A. Gray

I 2746 Harbourii A. Gray

I 2747 Havardi A. Gray

I 2748 Haydeni Wats.

I 2749 Helleri Small

I $275^{\circ}$ heterodoxus A. Gray

I 275 I heterophyllus Lindl.

I 2752 hirsutus (L。) Willd.

(P. pubescens Soland.)

I 2753 humilis Nutt.

I.2754 brevifolius A. Gray

12755 incertus T. S. Brandegee

I 2756 Jamesii Benth.

I2757 Kingii Wats.

I $275^{8}$ labrosus Hook. f.

12759 laetus A. Gray

I2760 leptosepalus Greene

1276 I lanceolatus Benth.

12762 laticifolius H. \& A.

12763 Lemmoni A. Gray

12764 leucanthus Greene

I 2765 linarioides A. Gray

12766 Sileri A. Gray

12767 Ilnearifolius Coult. \& Fisher

12768 Lyallii A. Gray

12769 Menziesii Hook.
I2770 Scouleri (Lindl.) A. Gray

1277 I micranthus Nutt.

I 2772 microphyllus A. Gray

I 2773 miser A. Gray

I 2774 Moffattii Eastwood

I 2775 montanus Greene

r 2776 Murrayanus Hook.

I 2777 nemorosus (Dougl.) Traut.

12778 Newberryi A. Gray

I 2779 rupicola Piper

I 2780 nudiflorus A. Gray

I 278I ovatus Dougl.

I 2782 Palmeri A. Gray

I 2783 Parishii A. Gray

I 2784 Parryi A. Gray

12785 Pentstemon (L.) Britton

(P. laevigatus Soland.)

I 2786 petiolatus T. S. Brandegee

I 2787 pseudohumilis Rydb.

I 2788 pseudoprocerus Rydb.

I 2789 pinifolius Greene

I 2790 procerus Dougl.

( $P$. confertus var.coerulea-purpureus)

I279I pruinosus Dougl.

I 2792 pulchellus Greene

I 2793 pumilus Nutt.

I 2794 incanus A. Gray

I 2795 Thompsoniae A. Gray

12796 puniceus A. Gray

I 2797 Purpusi T. S. Brandegee

I 2798 radicosus Aven Gelson

I 2799 Rattani A. Gray

I 2800 Kleei A. Gray

I280I minor A. Gray

I 2802 Richardsonii Dougl.

I 2803 riparius Aven Nelson

I 2804 Roezli Regel

I 2805 violaceus 'T. S. Brandegee

I 2806 Rothrockii A. Gray

I 2807 Rydbergii Aven Nelson

I 2808 saliens Rydb.

I 2809 Scouleri Dougl.

I28ro secundiflorus Benth.

I 28I I Shockleyi Wats.

I 28 I 2 similis Aven Nelson

I 2813 Smallii Heller

I 28I 4 Sonomensis Greene

I 28 I 5 speciosus Dougl. 

I 28 I6 spectabilis Thurber

I 28 I 7 stenophyllus A. Gray.

I 28 I 8 dasyphyllus A, Gray

I 2819 strictus Benth.

I 2820 sudans Jones

I 282 I ternatus Torr.

12822 Torreyi Benth.

I 2823 triflorus Heller

I 2824 triphyllus Dougl.

I 2825 tubiflorus Nutt.

I 2826 Utahensis(Wats.)A venNelson I 2863

I 2827 venustus Dougl.

I 2828 virgatus A. Gray

I 2829 Wardii A. Gray

I 2830 Watsoni A. Gray

I283I Whippleanus A. Gray

I 2832 Whitedii Piper

I 2833 Wrightii Hook.

\section{Chionophila}

I 2834 Jamesii Benth.

I 2835 Tweedyi (Canby \& Rose)

Henderson

(Pentstemon Tweedyi Canby \& Rose)

\section{Paulownia}

I 2836 tomentosa (Thurb.) Baill. (P. imperialis Sieb. \& Zucc.)

\section{Mimetanthe}

I 2837 pilosa (Benth.) Greene (Mimulus pilosus Wats.)

\section{Mimulus}

I 2838 acutidens Greene

I 2839 alatus Soland.

I 2840 alsinoides Benth.

I284I arvensis Greene

I 2842 atropurpureus Kellogg

I 2843 bicolor Hartw.

I 2844 caespitosus Greene

I 2845 cardinalis Dougl.

I 2846 corallinus Greene

I 2847 deflexus Wats.

I 2848 dentatus Nutt.

I 2849 exilis Durand

I 2850 filicaulis Wats.

I 285I floribundus Dougl.

I 2852 glabratus H. B. K.

I 2853 adscendens A. Gray
I 2854 glareosus Greene

I 2855 glancescens Greene

I 2856 gracillipes Robinson

I 2857 guttatus DC.

I 2858 implexus Greene

I 2859 inconspicuus A. Gray

I2860 Jamesii T. \& G.

I 286 I laciniatus A. Gray

I 2862 I angsdorfii Sims

(M. luteus Pursh, not $\mathrm{I}_{\text {. }}$ ) argutus Greene grandis Greene insignis Greene platyphyllus Greene Tilingi (Regel) Greene

I 2867 I 2868 latidens (A. Gray) Greene I2869 Lewisii Pursh

12870 exsertus Coult. \& Fisher

I 287 I marmoratus Greene

12872 moniliformis Greene

I 2873 montioides A. Gray

I 2874 moschatus Dougl.

I 2875 longiflorus A. Gray

I 2876 sessiliflor us A. Gray

I 2877 nasutus Greene

r 2878 nudatus Curran

I 2879 Palmeri A. Gray

I 2880 androsaceus A. Gray

I288I Parishii Greene

I 2882 peduncularis Dongl.

I 2883 pilosellus Greene

I 2884 primuloides Benth.

I 2885 Pulsiferae A. Gray

I 2886 ringens L.

I 2887 rubellus A. Gray

I 2888 Scouleri Hook.

I 2889 subreniformis Greene

I 2890 Suksdorfii A. Gray

I 289I thermalis Aven Nelson

I 2892 Whitneyi A. Gray

\section{Diplacus}

(Mimulus in part)

I 2893 Clevelandi (Brandegee)

I 2894 glutinosus Nutt. Grcer:e

I 2895 grandiflorus Greere

I 2896 latifolius Nutt.

I 2897 linearis (Benth.) Greene 

I2898 longiflorus Nutt.

I 2899 parviflorus Greene

12900 puniceus Nutt.

I 290 I stellatus Kellogg

I 2902 speciosus Davy

\section{Eumanus}

(Mimulus in part)

12903 angustatus (A. Gray) Greene I 2904 Austinae Greene

I 2905 Bigelovii A. Gray

12906 Bolanderi (A. Gray) Greene

I 2907 brevipes Benth.

I 2908 Breweri Greene

I 2909 Congdoni (Robinson) Greene

I 2910 Cusickii Greene

I 29I I Douglasii Benth.

I 2912 parviflorus Greene

r2913 Fremontii Benth.

I 29I4 Kelloggii Curran

I 2915 latifolius (A. Gray) Greene

I29I6 Laynae Greene

I 2917 leptaleus (A. Gray) Greene

12918 mephiticus Greete

I 2919 Mohavensis(Lemmon)Greene I 2920 nanus (H. \& A.) Holzinger

I 292 I Parryi (A. Gray) Greene

I 2922 pictus Curran

I 2923 pulchellus Drew

12924 Rattani (A. Gray) Greene

I 2925 subsecundus (A.Gray) Greene

I 2926 subuniflorus (H.\& A.) Greene

I 2927 Tolmiei Benth.

12928 Torreyi (A. Gray) Greene

I 2929 tricolor (Lindl.) Greene

\section{Stemodia}

I2930 durantifolia Sw.

I293I lanata R. \& P.

\section{Gratiola}

I 2932 aurea Muh1.

I 2933 Drummondii Benth.

I 2934 ebracteata Benth.

I 2935 Floridana Nutt.

12936 gracilis Benth.

I 2937 hispida (Benth.) Pollard

(G. subulata Baldwin)

$1293^{8}$ officinalis L.

I2939 pilosa Michx.
I 2940 pusilla Torr.

I 294 I ramosa Walt.

I 2942 sphaerocarpa Ell.

I 2943 Virginiana L.

I 2944 viscosa Schwein.

\section{Comobea}

I 2945 intermedia A. Gray

I 2946 multifida (Miclıx.) Benth.

\section{Monniera}

(Herpestis)

I 2947 acuminata (Walt.) Kuntze (H. nigrescens Benth.)

I 2948 Caroliniana (Walt.) Kuntze (H. amplexicaulis Pursh)

I 2949 crenulata Small

I $295^{\circ}$ Monniera (L.) Britton

I 295I procumbens (Mill.) Kuntze (H. chamaedryoides H.B.K.)

I 2952 repens (Sw.) Kuntze

I 2953 rotundifolia Michx.

\section{Micranthemum}

I 2954 emarginatum Ell.

I 2955 micranthemoides (Nutt.)

Wettst.
(M. Nuttallii A. Gray)

I 2956 orbiculatum Michx.

\section{Amphianthus}

I 2957 pusillus Torr.

\section{Limosella}

I $295^{8}$ aquatica L.

I 2959 tenuifolia Hoffm.

\section{Ilysanthes}

I 2960 attenuata (Muhl.) Small

I 296 I dubia (L.) Barnhart

(I. gratioloides Benth.)

I 2962 grandiflora (Nutt.) Benth.

12963 refracta (E11.) Benth.

I 2964 saxicola (M.A.Curtis) Chapm.

12965 tridentata Small

\section{Capraria}

I 2966 biflora L.

\section{Scoparia}

I 2967 dulcis L.

I 2968 grandiflora Nash 



\section{Veronica}

I 2969 agrestis L.

12970 Allenii Greenman

I297 I Americana Schwein.

I 2972 crassula Rydb.

I 2973 Anagallis-aquatica L.

I 2974 arvensis L.

I.2975 Byzantina (S. \& S.) B. S. P. (V. Buxbaumii Tenore)

I 2976 chamaedrys L.

12977 Cusickii A. Gray

I 2978 hederaefolia L.

12979 Kantchatica L. f.

I 2980 officinalis L.

I 298 I peregrina L.

I 2982 scutellata L.

I 2983 pubescens Macoun

I 2984 serpyllifolia I.

I 2985 Stelleri Pall.

I 2986 Wormskjoldii R. \& S.

( $V$. alpina of anthors, not L.)

\section{Leptandra}

I 2987 Virginica (L.) Nutt. (Veronica Virginica L.)

\section{Lagotis}

12988 glauca Gaertn.

\section{Synthyrys}

(Wulfenia Greene, not Jacq.) 12989 alpina A. Gray

r 2990 Bullii (Eaton) Heller

(S. Houghtoniana Benth.)

I 299 I cordata (A. Gray) Heller

I 2992 gymnocarpa (Aven Nelson)

I 2993 laciniata (A. Gray) Rydb.

Heller

I 2994 major (Hook.) Heller

I 2995 pinnatifida Wats.

I 2996 plantaginea Benth.

I 2997 reflexa Eastwood

I 2998 reniformis Dougl.

I 2999 Ritteriana Eastwood

I 3000 rotundifolia A. Gray

I 3001 rubra (Dougl.) Benth.

I 3002 Wyomingensis (AvenNelson)

\section{Digitalis}

Heller

I 3003 purpurea I.

\section{Macranthera}

I 3004 fuchsioides 'Torr.

I3005 Lecontei Chapm.

\section{Afzelia}

(Seymeria)

I 3006 bipinnatisecta(Seem.)Kuntze

3007 cassinoides (Walt.) Gmel.

(S. tenuifolia Pursh)

I 3008 macrophylla (Nutt.) Kuntze

I 3009 pectinata (Pursh) Kuntze

I 3010 scacra (A. Gray) Kuntze

I 3 OI I virgata (H. B. K.) Kuntze

\section{Gerardia}

I 3012 aphylla Nutt.

I 3013 aspera Dougl.

I30I4 auriculata Michx.

I 3 OI 5 Besseyana Britton

I 3016 decemloba Greene

I 3017 densiflora Benth.

I 3018 divaricata Chapm.

I 3019 filicaulis Chapm.

I 3020 filifolia Nutt.

I 302 I heterophylla Nutt.

I3022 Holmiana Greene

r 3023 lancifolia Greene

I3O24 linifolia Nutt.

I 3025 maritima Raf.

I 3026 patula Chapm.

I 3027 paupercula (A. Gray) Britton

I 3028 Plukenetti Ell.

I 3029 microphylla A. Gray

I 3030 polyphylla Sinall

I 303 I purpurea L.

I 3032 fasciculata Chapm.

I 3033 setacea Walt.

I 3034 longifolia A. Gray

I 3035 Skinneriana Wood.

(G. parvifolia Chapm.)

I3036 strictiflora Benth.

I 3037 tenuifolia Vahl.

I 3038 asperula A. Gray

I 3039 viridis Small

I 3040 Wrightii A. Gray

\section{Dasystoma}

(Gerardia in part)

I 304I bignoniifolia Small 

I 3042 flava (L.) Wood

I 3043 grandiflora (Benth.) Wood

13044 laevigata Raf.

I 3045 pectinata (Nutt.) Benth.

I 3046 Pedicularia (L.) Britton

I 3047 Virginica (L.) Britton (G. quercifolia Benth.)

\section{Buchnera}

I3048 Americana L.

r 3049 elongata Sw.

I 3050 lithospermifolia H. B. K.

I 305 I pilosa Arizonica A. Gray

\section{Castilleja}

I 3052 acuminata (Pursh) Spreng (C. pallida var. septentrionalis Gray) I 3053 affinis H. \& A.

I 3054 angustifolia (Nutt.) Don

I3055 abbreviata Fernald

I 3056 adenophora Fernald

I 3057 Whitedii Piper

I $305^{8}$ Applegatei Fernald

I 3059 brachyantha Rydb.

(C. breviflora A. Gray)

I3060 Bradburii (Nutt.) Don

I 306 I Breweri Fernald

I 3062 cervina Greenman

I 3063 chromosa Aven Nelson

I 3064 cinerea A. Gray

I 3065 coccinea (L.) Spreng.

I 3066 confusa Greene

I3067 Covilleana Henderson

13068 Cristangalli Rydb.

I 3069 Cusickii Greenman

I 3070 Dixoni Fernald

I307I Elmeri Fernald

I 3072 fasciculata Aven Nelson

I 3073 flava Wats.

I 3074 foliolosa H. \& A.

I 3075 gloriosa Britton

I 3076 Haydeni (A. Gray) Cockerell

I 3077 hispida Benth.

I 3078 hololenca Greene

I 3079 indivisa Engelm.

I 3080 integra A. Gray

I 308 I gracilis Cockerell

I3082 lanata A. Gray

I 3083 lanceifolia Rydb.
I 3084 latifolia H. \& A.

I 3085 lauta Aven Nelson

I3086 laxa A. Gray

I 3087 Lemmoni A. Gray

I 3088 leviseta Greenman

I3089 linariaefolia Benth.

I 3090 Lindheimeri A. Gray

I309I lineata Greene

I 3092 linoides A. Gray

I 3093 longispica Aven Nelson

I 3094 lutea Heller

I 3095 lutescens (Greenman) Rydb.

I3096 miniata Dougl.

I 3097 minor A. Gray

r 3098 oblongifolia A. Gray

I 3099 occidentalis Torr.

I 3100 oreopola Greenman

I 3 IOI subintegra Fernald

I 3 IO2 pallescens (A. Gray) Greenm.

(Orthocarpus pallescens A. Gray)

I 3 IO3 pallida Kunth.

I3IO4 camporum Greenman

I 3105 parviflora Bong.

I 3106 pilosa (Wats.) Rydb.

(Orthocarpus pilosus Wats.)

I3107 pinetorum Fernald.

I 3108 plagiotoma A. Gray

I 3 I09 pruinosa Fernald

I 3 I IO purpurea Don

I 3 I I I remota Greene

I3II2 rhexifolia Rydb.

I 3 II 3 rubida Piper

I 3 II4 rupicola Piper

I3II5 rustica Piper

I 3 I 6 sessiliflora Pursh

I 3 II 7 stenantha A. Gray

I 3 I 8 stricta Piper

I 3 I 9 subinclusa Greene

I 3 I 20 Suksdorfii A. Gray

I3I I sulphurea Rydb.

I3I22 Tweedyi Rydb.

I 3 I 23 villosa Rydb.

I 3 I 24 viscidula A. Gray

I3I25 Watsoni Heller

\section{Adenostegia}

(Cordylanthus)

I 3 I 26 canescens (A. Gray) Greene 

I 3I27. capitata (Nutt.) Greene I 3I 28 Kingii (Wats.) Greene I 3 I 29 laxiflora (A. Gray) Greene I3 I30 maritima (Nutt.) Greene I3I 3 I mollis (A. Gray) Greene I 3 I 32 Nevinii (A. Gray) Greene I 3 I 33 Parryi (A. Gray) Greene I 3 I 34 pilosa (A. Gray) Greene 13I35 Bolanderi(A.Gray)Greene I 3 I 36 Pringlei (A. Gray) Greene I $3^{\text {I } 37 \text { ramosa (Nutt.) Greene }}$ I 3 I 38 rigida Benth. I3I39 brevibracteata (A. Gray) Greene

I 3 I 40 tenuis (A. Gray) Greene I3I4I Wrightii (A. Gray) Greene

\section{Orthocarpus}

I3I42 attenuatus A. Gray

I 343 Bidwelliae A. Gray I3 44 micranthus A. Gray

I3I 45 bracteosus Benth.

I 3146 campestris Benth.

I 3147 castilleoides Benth.

I 3 I 48 Columbianus Jones

I3I49 cuspidatus Greene

I 3 I 50 densiflorus Benth.

I3I5I erianthus Benth.

I 3 I 52 faucibarbatus A. Gray

I 3 I53 floribundus Benth.

I 154 gracilis Benth.

I 355 hispidus Benth.

I 3 I 56 imbricatus Torr.

I 3 I57 lacerus Benth.

I $3^{1} 5^{8}$ lasiorhynchus A. Gray

13I59 linearilobus Benth.

I3I60 lithospermoides Benth.

I3I6I luteus Nutt.

I 3 I62 pachystachys A. Gray

I 3 I63 Parishii A. Gray

I 3 I64 purpurascens Benth.

I3I65 Palmeri A. Gray

13I66 purpureo-albus A. Gray

I 3167 pusillus Benth.

I3I68 tenuifolius Benth.

I3r69 Tolmiei H. \& A.

I3I 70 versicolor (F. \& M.) Greene I 3I 7 I roseus (A. Gray) Greene

\section{Melampyrum}

I3I72 lineare Lam. (M. Americanum Michx.)

I3I73 latifolium Muhl.

\section{Euphrasia}

I3I74 Americana Wettst.

I3I75 latifolia Pursh

I3I 76 Oakesii Wettst.

[E. officinalis does not occur in this country]

\section{Odontites}

I3I 77 Odontites (L.) Wettst. (Bartsia Odontites Huds.)

\section{Bartsia}

I 3 7 8 alpina $\mathrm{L}$.

\section{Pedicularis}

I 3 I 79 albomarginata Jones

I 3I80 bracteosa Benth.

I3I8I Canadensis L.

I 3 I82 Canbyi A. Gray

I3I83 capitata Adams

I 3 I84 centranthera A. Gray

I3I85 Chamissonis Stev.

I 3 I 86 contorta Benth.

I3I87 crenulata Benth.

I 3I 88 ctenophora Rydb.

I 3 I89 cystopteridifolfa Rydb.

I 3 I90 densiflora Benth.

I3I9I euphrasioides Stephan

I 3192 flammea L.

I 3 I93 fluviatilis Heller

I 3 I94 Furbishiae Wats.

I3I95 Hallii Rydb.

I 3196 hirsuta L.

I3I97 Howellii A. Gray

I3I98 lanceolata Michx.

I 3199 Langsdorfli Fisch.

I 3200 lanata A. Gray

I 320 I Lapponica L.

I 3202 Menziesii Benth.

I $32 \mathrm{O} 3$ Montanensis Rydb.

I 3204 ornithorhyncha Benth.

I 3205 palustris L.

I3206 Parryi A. Gray

I 3207 parviflora J. E. Smith

I3208 pedicellata Bunge 

I 3209 procera Bunge

I3210 racemosa Dougl.

I32I I scopulorum A. Gray

I 3212 semibarbata A. Gray

I32I 3 Suedetica Willd.

I32I 4 versicolor Wahl.

I 32 I 5 verticillata $L$.

\section{Elephantella}

(Pedicularis in part)

13216 attolens (A. Gray) Heller

I3217 Groenlandica (Retz.) Rydb.

Fistularia

(Rhinanthus)

I32 8 Crista-galli (L.) Wettst.

Schwalbea

I32I9 Americana L.

\section{LENTIBULARIACEAE}

Pinguicula

I 3220 alpina L.

I322I elatior Michx.

I3222 lutea Walt.

I 3223 edentula A. DC.

I3224 pumila Michx.

I 3225 villosa $L$.

I3226 vulgaris L.

\section{Utricularia}

13227 biflora Lam.

I 3228 bipartita Ell.

I3229 clandestina Nutt.

I 3230 cleistogama (A.Gray) Britton

I323I cornuta Michx.

I 3232 fibrosa Walt.

I 3233 Floridana Nash

I 3234 gibba L.

I 3235 inflata Walt.

I 3236 intermedia Hayne

I 3237 juncea Vahl.

r 3238 longiciliata A. DC.

I 3239 macrorhyncha Barnhart

I 3240 minor L.

I 324 I occidentalis A. Gray

I 3242 oligosperma St. Hil.

I 3243 purpurea Walt.

I3244 resupinata B. D. Greene

I3245 simplex C. Wright
I3246 subulata L.

I 3247 vulgaris L.

\section{OROBA NCHACEAE}

\section{Conopholis}

I 3248 Americana (L. f.) Wallr.

I3249 Mexicana A. Gray

\section{Orobanche}

(Aphyllon in part)

I 3250 Californica C. \& S.

I 325 I comosa Hook.

I 3252 Cooperi (A. Gray) Heller

I 3253 Ludoviciana Nutt.

I 3254 minor J. E. Smith.

I 3255 multiflora Nutt.

I 3256 pinetorum Geyer

I 3257 ramosa $\mathrm{L}$.

I $325^{8}$ tuberosa (A. Gray) Heller

\section{Thalesia}

(Aphyllon)

I3259 fasciculata (Nutt.) Britton

I3260 lutea (Parry) Britton

I326I purpurea Heller

I 3262 uniflora (L.) Britton

\section{Leptamnium}

(Epiphegus)

I 3263 Virginianum (L.) Raf.

\section{Boschniakia}

I3264 glabra C. A. Meyer

I 3265 Hookeri Walp.

I3266 strobilacea A. Gray

\section{BIGNONIACEAE}

\section{Bignonia}

I 3267 crucigera L.

(B. capreolata $\mathrm{L}_{10}$ )

\section{Chilopsis}

I 3268 linearis (Cav.) Sweet (C. saligna Don)

\section{Catalpa}

I 3269 Catalpa (L.) Karst. (C. bignonioides Walt.)

I 3270 speciosa Warder 



\section{Campsis}

I 327 I radicans (L.) Seem. (Tecoma radicans DC.)

Stenolobium

I 3272 stans (L.) D. Don (Tecoma stans Juss.)

Crescentia

I 3273 ovata Burmann

PEDALIACEAE

\section{Sesamum}

I3274 Indicum L.

\section{MARTYNIACEAE}

\section{Martynia}

r 3275 althaefolia Benth.

I 3276 fragrans Lindl.

I 3277 Louisiana Mill.

(M. proboscidea Glox.)

I3278 parviflora Wooton

\section{ACANTHACEAE}

\section{Tubiflora}

(Elytraria)

I 3279 Carolinensis (Walt.) Gmel. (E. virgata Michx.)

I 3280 squamosa (Jacq.) Kuntze (E. tridentata Vahl.)

\section{Hygrophila}

I328r lacustris Nees

\section{Ruellia}

I 3282 ciliosa Pursh

I 3283 parviflora (Nees) Britton I 3284 clandestina L. (R. tuberosa L.)

13285 Drummondiana A. Gray

I 3286 graminiflora H. B. K.

I 3287 humilis Nutt.

I 3288 noctiflora A. Gray

13289 Parryi A. Gray

I 3290 pedunculata Torr.

r329I strepens L.

I 3292 micrantha(Engelm.\&Gray)

Calophanes

I3293 angusta A. Gray
I 3294 decumbens A. Gray

I 3295 humistrata Nees

I 3296 linearis ('T. \& G.) A. Gray

r 3297 oblongifolia (Miclıx.) Don

\section{Stenandrium}

I 3298 barbatum T. \& G.

I 3299 dulce Nees

I 3300 Floridanum A. Gray

\section{Berginia}

I330I virgata Harvey

\section{Carlowrightia}

13302 Arizonica A. Gray

I3303 linearifolia A. Gray

I 3304 pubens A. Gray

\section{Anisacanthus}

I 3305 insignis A. Gray

I 3306 Thurberi (Torr.) A. Gray

I 3307 Wrightii ('Torr.) A. Gray

\section{Tetramerium}

I3308 hispidum Nees

I3309 platystegium Torr.

\section{Diapedium}

(Dicliptera)

I33IO assurgens (L.) Kuntze

I33 I attenuatum (A. Gray) Heller

I 33I 2 brachiatum (Pursh) Kuntze

I33 3 resupinatum (Vahl.) Kuntze

I33I 4 Torreyi (A. Gray) Heller

\section{Siphonoglossa}

I 33 I 5 dipteracantha (Nees) Heller (S. Pilosella Torr.)

I3316 longiflora (Torr.) A. Gray

\section{Justicia}

I33I7 Wrightii A. Gray

\section{Dianthera}

I3318 Americana L.

I3319 crassifolia Chapm.

I 3320 ovata Walt.

(D. humilis Engelm. \& Gray)

I332I parviflora A. Gray

I 3322 parvifolia B. \& H.

\section{Jacobinia}

I3323 neglecta A. Gray 



\section{Beloperone}

I3324 Californica Benth.

\section{Yeatesia}

(Gatesia)

I 3325 laete-virens (Buckley) Small

\section{PHRYMACEAE}

\section{Phryma}

I 3326 Leptostachya L.

\section{PLANTAGINACEAE}

\section{Plantago}

I 3327 argyrea Morris

13328 aristata Michx.

I 3329 Asiatica L.

I 3330 Bigelovii A. Gray

I333I brunnea Morris

I 3332 cordata Lam.

I3333 decipiens Barneoud

I 3334 dura Morris

I3335 elongata Pursh

I3336 erecta Morris

I3337 eriopoda Torr.

I 3338 fastigiata Morris

r3339 Helleri Sinall

I 3340 heterophylla Nutt.

I 334 I hirtella H. B. K.

I 3342 inflexa Morris

I 3343 insularis Eastwood

I 3344 lanceolata L.

I 3345 lanatifolia (Coult. \& Fisher)

I 3346 macrocarpa C. \& S. Small

I 3347 major L.

I3348 maritima L.

I3349 media I.

I $335^{\circ}$ Mexicana Link.

I $335^{\text {I }}$ myosuroides Rydb.

I $335^{2}$ Nuttallii Rapin

I 3353 Purshii R. \& S.

(P. Patagonica var. gnaphaloides)

I3354 pusilla Nutt.

I 3355 Rugelii Dcne.

13356 scariosa Morris

I3357 sparsiflora Michx.

I 3358 spinulosa Dcne.

I 3359 tetrantha Morris
I3360 Tweedyi A. Gray

I336I Virginica L.

r3362 longifolia A. Gray

I3363 Wrightiana Dcne.

(P. Patagonica var. nuda A. Gray)

\section{Littorella}

I3364 uniflora (I.) Rusby

(L. lacustris L.)

\section{RUBIACEAE}

\section{Pinckneya}

I3365 pubens Michx.

\section{Oldenlandia}

I3366 Boscii (DC.) Chapm.

I 3367 littoralis Mohr

I 3368 uniflora L.

(O. glomerata Michx.)

\section{Houstonia}

I3369 acerosa A. Gray

I3370 angustifolia Michx.

I337 I filifolia A. Gray

I 3372 rigidiuscula A. Gray

r 3373 ciliolata Torr.

I 3374 coerulea L.

I 3375 Croftiae Britton \& Rusby

I 3376 fasciculata A. Gray

I 3377 humifusa A. Gray

I 3378 longifolia Gaertn.

I 3379 minima Beck

I 3380 minor (Michx.) Britton (H.patens E11.)

I338I pulvinata Small

I 3382 purpurea L.

I 3383 calycosa A. Gray

I3384 pubescens Britton

I3385 rotundifolia Michx.

I $33^{86^{\circ}}$ rubra Cav.

I 3387 salina Heller

I3388 serpyllifolia Michx.

I3389 subviscosa A. Gray

I3390 tenuifolia Nutt.

I3391 Wrightii A. Gray

\section{Pentodon}

I 3392 Halei ('T. \& G.) A. Gray 



\section{Bouvardia}

13393 angustifolia H. B. K.

I3394 ovata A. Gray

I3395 triphylla Salisb.

\section{Catesbaea}

r 3396 parviflora Sw.

\section{Exostema}

I 3397 Caribaeum (Jacq.) R. \& S.

\section{Cephalanthus}

I3398 occidentalis L.

\section{Randia}

I3399 aculeata I.

\section{Genipa}

I3400 clusiaefolia (Jacq.) Griseb.

\section{Hamelia}

I340I patens Jacq.

\section{Guettarda}

I 3402 elliptica Sw.

I 3403 scabra (L.) Lam.

\section{Erithalis}

I 3404 fruticosa I.

\section{Chiococea}

I3405 racemosa L.

\section{Strumpfia}

I3406 maritima Jacq.

\section{Psychotria}

I 3407 tenuifolia Sw.

I 3408 undata Jacq.

\section{Kelloggia}

${ }_{1} 3409$ galioides Torr.

\section{Mitchella}

I34Io repens L.

\section{Morinda}

I34II Roioc L.

\section{Richardia}

I 34 I 2 Brasiliensis Gomez

I 34 I 3 scabra L.

\section{Ernodea}

I34 4 littoralis Sw.

\section{Crusea}

I34I 5 subulata (DC.) A. Gray

I34I6 tricocca ('T. \& G.) Heller (C. alococca A. Gray)

134I7 Wrightii A. Gray

\section{Diodia}

I 34I 8 hirsuta Pursh

I34I9 teres Walt.

I3420 angustata A. Gray

I342 I tetragona Walt.

13422 Virginiana L.

\section{Spermacoce}

I3423 glabra Michx.

I3424 parviflora (DC.) A. Gray

I 3425 podocephala (DC.) A. Gray

I 3426 Portoricensis Balbis

I 3427 tenuior Lam.

\section{Borreria}

I3428 verticillata (L.) Mey.

\section{Mitracarpus}

I 3429 breviflorus A. Gray

\section{Sherardia}

I 3430 arvensis L.

Asperula

I343I odorata L.

\section{Galium}

I3432 Andrewsii A. Gray

I3433 angustifolium Nutt.

I3434 Aparine L.

I 3435 arcuatnm Wiegand

I3436 Arkansanum A. Gray

I3437 asperrimum A. Gray

I $343^{8}$ asprellum Michx.

I3439 bifolium Wats.

I 3440 Bolanderi A. Gray

I 344 I boreale L.

I3442 linearifolium Rydb.

I 3443 Brandegei A. Gray

I3444 buxifolium Greene

r 3445 Californicum H. \& A.

I 3446 Catalinense A. Gray

I 3447 circaezans Michx.

I3448 glabellum Britton

r3449 Claytoni Michx. 

I $345^{\circ}$ concinnum T. \& G.

I 345 I cymosum Wiegand

I $345^{2}$ Fendleri A. Gray

I 3453 flaccidum Greene

I 3454 grande McClatchie

I 3455 hispidulum Michx.

I 3456 Kamptschaticum Steller

I 3457 lanceolatum Torr.

I $345^{8}$ latifolium Michx.

I3459 hispidifolium Small

I 3460 Matthewsii A. Gray

I346I microphyllum A. Gray

I 3462 Miguelense Greene

I 3463 Mollugo L.

I 3464 multiflorum Kellogg

I 3465 hirsutum A. Gray

I3466 Nuttallii A. Gray

I 3467 occidentale McClatchie

I 3468 Oreganum Britton

I 3469 palustre L.

I 3470 Parisiense L.

(G. Anglicum Huds.)

I 347 I pilosum Ait.

I 3472 puncticulosum (Michx.)

I 3473 proliferum A. Gray T. \& G.

I 3474 pubens A. Gray

I 3475 Rothrockii A. Gray

I3476 stellatum Kellogg

I 3477 sylvaticum L.

I 3478 sylvestre Poll.

I3479 Texense A. Gray

I 3480 tinctorium I.

I 348 I filifolium Wiegand

I 3482 Floridanum Wiegand

I 3483 Labradoricum Wiegand

I 3484 tricorne Stokes

I 3485 trifidum L.

I 3486 Pacificum Wiegand

I 3487 subbiflorum Wiegand

I 3488 triflorum Michx.

I3489 uniflorum Michx.

I 3490 Vaillantii DC.

r 349 r verum L.

I 3492 virgatum Nutt.

I 3493 leiocarpum T. \& G.

I 3494 Watsoni (A. Gray) Heller
I3495 Wrightii A. Gray

\section{CAPRIFOLIACEAE}

Sambucus

I 3496 arborescens (T. \& G.) Nutt.

I 3497 callicarpa Greene

I 3498 Canadensis L.

I3499 laciniata (Mill.) A. Gray

I 3500 glauca Nutt.

I35OI leiosperma Leiberg

I 3502 maritima Greene

I35O3 melanocarpa A. Gray

I3504 Mexicana Presl.

I 3505 Neo-Mexicana Wooton

I 3506 pubens Michx.

I 3507 dissecta Britton

\section{Viburnum}

I 3508 acerifolium L.

I3509 alnifolium Marsh.

( $V$. lantanoides Michx.)

I 35 IO cassinoides L.

I35I I Demetrionis Deane \& Rob.

I 3512 densiflorum Chapm.

I35I3 dentatum L.

I35I 4 ellipticum Hook.

I 35 I 5 Lentago L.

I 35 I 6 molle Michx.

I 35 I 7 nitidum Ait.

I 35 I 8 nudum L.

I 3519 angustifolium T. \& G.

I 3520 quadrifolium A. Gray

I 352 I serotinum Ravenel

I 3522 obovatum Walt.

13523 Opulus L.

I 3524 pauciflorum Pylaie

I 3525 prunifolium L.

I 3526 globosum Nash

I 3527 pubescens (Ait.) Pursh

I 3528 petiolatum Fitzpatrick

r 3529 rufotomentosum Small

\section{Triosteum}

I 3530 angustifolium L.

I353I perfoliatum L.

\section{Symphoricarpos}

13532 acutus (A. Gray) Howell

I 3533 longiflorus A. Gray 

I3534 mollis Nutt.

I 3535 occidentalis Hook.

I 3536 Heyeri Dieck.

I3537 oreophilus A. Gray

I 3538 Parishii Rydb.

I 3539 pauciflorus (Robbins) Britton

13540 racemosus Michx.

I354I rotundifolius A. Gray

I 3542 spicatus Engelm.

I3543 Symphoricarpos (L.) MacM. (S. vulgaris Michx.)

I 3544 Utahensis Rydb.

I 3545 vaccinioides Rydb.

\section{Limna'ea}

13546 borealis L.

r 3547 longiflora ('Torr.) Howell

\section{Lonicera}

I 3548 albiflora T. \& G.

I 3549 Californica T. \& G

I $355^{\circ}$ Canadensis Marsh.

(L. ciliata Muhl.)

I355I Caprifolium L.

I $355^{2}$ ciliosa (Pursh) Poir.

I 3553 conjugialis Kellogg

I 3554 dioica L.

$$
\text { (L. glauca Hill) }
$$

I 3555 ebractulata Rydb.

I 3556 flava Sims

I 3557 glaucescens Rydb.

I $355^{8}$ hirsuta Eaton

I 3559 hispidula (Lindl.) Dougl.

I 3560 interrupta Benth.

I 356 I involucrata (Rich.) Banks

I 3562 Japonica Thunb.

${ }_{1} 3563$ Ledebourii Esch.

I 3564 oblongifolia (Goldie) Hook.

I 3565 sempervirens $L$.

I 3566 subspicata H. \& A.

I 3567 Sullivantii A. Gray

I 3568 Tatarica L.

13569 Utahensis Wats.

I 3570 villosa Michx.

(L. coerulea of authors, not L.) I357I Xylosteum L.

\section{Diervilla}

I 3572 Diervilla (L.) MacM. (D. trifida Moench)
I 3573 rivularis Gattinger

I 3574 sessilifolia Buckley

\section{ADOXACEAE}

\section{Adoxa}

I3575 Moschatellina L.

\section{VALERIA NACEAE \\ Plectritis}

(Valerianella in part)

13576 anomala (A. Gray) Suksdorf

I 3577 aphanoptera (A. Gray) Suksd.

I 3578 brachystemon F. \& M.

I 3579 congesta (Lindl.) DC.

I 3580 involuta Suksdorf

I 3581 magna (Greene) Suksdorf

I $35^{82}$ major (F. \& M.) Hoeck

I $35^{8} 3$ microptera Suksdorf

I3584 samolifolia (DC.) Hoeck

\section{Aligera}

I $35^{8} 5$ ciliosa (Greene) Suksdorf

I3586 Eichleriana Suksdorf.

I 3587 Grayi Suksdorf

I3588 insignis Suksdorf

I 3589 Jepsoni Suksdorf

I 3590 macroptera Suksdorf

I 359I mamillata Suksdorf

I 3592 minor (Hook.) Heller

(Valerianella macrocera A Gray)

I3593 ostiolata Suksdorf

I3594 rubens Suksdorf

\section{Valerianella}

I3595 amarella (Lindheimer) Krok

I 3596 chenopodifolia (Pursh) DC.

I 3597 Locusta (L.) Bettke ( $V$. olitoria Poll.)

I 3598 longiflora ('T. \& G.) Walp.

I 3599 Nuttallii ('T. \& G.) Walp.

I 3600 radiata (L.) Dufr.

I 360 r stenocarpa (Engelm.) Krok

r 3602 Woodsiana (T. \& G.) Walp.

I 3603 pattelaria (Sulliv.)A.Gray

I 3604 umbilicata(Sulliv)A.Gray

\section{Valeriana}

I 3605 Arizonica A. Gray

I 3606 capitata Pall. 

I 3607 Columbiana Piper

I 3608 edulis Nutt.

I 3609 micrantha Elias Nelson

r 36 Io occidentalis Heller

I 36 I officinalis $\mathrm{L}$.

I 36 I 2 pauciflora Michx.

I 36 I 3 scandens L.

r36r 4 Scouleri Rydb.

I3615 septentrionalis Rydb.

( $V$. sylvatica Banks)

I36I6 Sitchensis Bong.

I 36 I 7 sorbifolia H. B. K.

I 36 I 8 Wyomingensis Elias Nelson

\section{DIPSACEAE}

\section{Dipsacus}

13619 Fullonum L.

I 3620 sylvestris Huds.

\section{Knautia}

I362I arvensis (L.) Coult. (Scabiosa arvensis L.)

\section{Scabiosa}

I 3622 atropurpurea L. I 3623 australis Wulf.

\section{CUCURBITACEAE}

\section{Apodanthera}

I 3624 undulata A. Gray

\section{Melothria}

I 3625 crassifolia Small

I 3626 Nashii Small

I 3627 pendula L.

13628 aspera Cogn.

r 3629 chlorocarpa (Engelm.)

I 3630 macrocarpa Cogn. Cogn.

\section{Ibervillea}

(Maximorviczia)

1 3631 Lindheimeri (Cogn.) Greene I 3632 tripartita (Cogn.) Greene

\section{Momordica}

I 3633 Balsamina L.

I 3634 Charantia I.

\section{Luffa}

I 3635 acutangula Roxb.

\section{Citrullus}

I 3636 Citrullus (L.) Small

(C. vulgaris Schrad.)

\section{Cucumis}

I 3637 Anguria L.

I 3638 Melo Texanus Nand.

\section{Lagenaria}

I 3639 Lagenaria (L.) Cockerell

(L. vulgaris Ser.)

\section{Cucurbita}

I 3640 Californica Torr.

I 364 I digitata A. Gray

I 3642 foetidissima H. B. K.

I 3643 palmata Wats.

I 3644 Pepo $\mathrm{L}$.

I 3645 verrucosa $L$.

\section{Cayaponia}

I 3646 Boykinii Cogn.

I 3647 grandifolia (T. \& G.) Small (Melothria grandifolia 'T. \& G.)

\section{Micrampelis}

(Megarrhiza)

I 3648 Gilensis (Greene) Britton

I3649 fabacea (Naud.) Greene

(M. Californica Torr.)

I 3650 agrestis Greene

I 365 I Guadalupensis (Wats.) Greene

I 3652 leptocarpa Greene

r 3653 lobata (Michx.) Greene

(Echinocystis lobata 'T. \& G.)

I 3654 macrocarpa Greene

I3655 Marah (Wats.) Greene

I 3656 Oregana (Torr.) Greene

I 3657 Watsoni (Cogn.) Greene (M. muricata Wats.)

\section{Echinopepon}

${ }_{13} 65^{8}$ horridus Naud.

I 3659 Wrightii Wats.

\section{Brandegea}

I3660 Bigelovii Cogn.

\section{Sicyos}

I 366I angulatus L.

I 3662 Deppei Don. 

I 3663 glaber Wooton r3664 laciniata subintegra Cogn.

\section{Sicyosperma}

I3665 gracile A. Gray

\section{Cyclanthera}

I 3666 dissecta ('T. \& G.) Arn.

\section{CAMPA N ULACEAE}

\section{Campanula}

I 3667 Americana L.

I3668 angustiflora Eastwood

I3669 aparinoides Pursh

I 3670 grandiflora Holzinger

I 367 I aurita Greene

I 3672 divaricata Michx.

I 3673 exigua Rattan

I 3674 Floridana Wats.

I 3675 glomerata L.

I 3676 lasiocarpa Cham.

I3677 linnaefolia A. Gray

I 3678 Parryi A. Gray

I3679 pilosa Pall.

I 3680 prenanthoides Durand

I 368 I rapunculoides I.

I 3682 Reverchoni A. Gray

I3683 rotundifolia L.

I3684 Alaskana A. Gray

I3685 Langsdorfiana (A. DC.)

(var. alpina Tuckerman)

Britton

I 3686 velutina $\mathrm{DC}$.

I3687 scabrella Eingelm.

I 3688 Scouleri Hook.

I 3689 uniflora L.

I3690 Wilkinsiana Greene

\section{Legouzia}

(Specularia)

I369I biflora (R. \& P.) Britton I 3692 Coloradoensis (Buckley)

\section{(S. Lindheimeri Vatke)} Heller

I 3693 leptocarpa (Nutt.) Britton I 3694 perfoliata (L.) Britton

\section{Heterocodon}

I3695 rariflorum Nutt.

\section{Githopsis}

I 3696 diffusa A. Gray

I 3697 specularioides Nutt.

Jasione

I 3698 montana L.

Sphenoclea

I3699 Zeylanica Gaertn.

\section{LOBELIACEAE}

\section{Nemacladus}

I 3700 longiflorus A. Gray

I370r ramosissimus Nutt.

I3702 montanus A. Gray

I37O3 pinnatifidus A. Gray

r 3704 rigidus Curran

\section{Parishella}

I 3705 Californica A. Gray

\section{Lobelia}

I3706 amoena Michx.

I3707 glandulifera A. Gray

I 3708 obtusata A. Gray

I 3709 appendiculata A. DC.

I37 Io Berlandieri A. DC.

I37II Boykini T. \& G.

I 37 I 2 brevifolia Nutt.

I37I3 Canbyi A. Gray

I 37 I 4 cardinalis L.

I 37 I 5 Cliffortiana L.

I37I 6 brachypoda A. Gray

I 37 I 7 Dortmanna L.

13718 Fieayana A. Gray

I37I9 fenestralis Cav.

I 3720 flaccidifolia Suall

I372I Gattingeri A. Gray

I 3722 glandulosa Walt.

13723 gruina Cav.

I 3724 hirtella (A. Gray) Greene

I 3725 inflata $L$.

I 3726 Kalmii L.

I3727 strictiflora Rydi.

I 3728 leptostachya A. DC.

I 3729 Ludoviciana (A.DC.) A. Gray

I 3730 Nuttallii R. \& S.

I 373 I paludosa Nutt.

I $373^{2}$ Floridana A. Gray 

I 3733 puberula Michx.

I3734 glabella Hook.

I3735 spicata Lam.

I 3736 parviflora A. Gray

I 3737 splendens Willd.

I $373^{8}$ syphilitica L.

I3739 Xalapensis H. B. K.

\section{Palmerella}

I 3740 debilis A. Gray

I 374I serrata A. Gray

I 3742 tenera A. Gray

\section{Porterella}

I 3743 carnosula (H. \& A.) Torr. (Laurentia carnosula Benth.)

I3744 eximia Aven Nelson

[Our American plants are no longer regarded as belonging to the OldWorld genus Laurentia]

\section{Bolelia}

\section{(Dozeningia)}

I 3745 bicornuta (A. Gray) Greene

I3746 brachyantha Rydb.

I 3747 concolor Greene

I 3748 cuspidata Greene

I3749 elegans (Dougl.) Greene

I3750 humilis Greene

I375I insignis Greene

r $375^{2}$ laeta Greene

I 3753 montana Greene

I3754 ornatissima Greene

I3755 pulchella (Lindl.) Greene

I 3756 pusilla (Poepp.) Greene

I3757 tricolor Greene

\section{Howellia}

I $375^{8}$ aquatilis A. Gray

I3759 limosa Greene

\section{GOODENIACEAE}

\section{Scaevola}

I 3760 Plummeri Vahl.

COMPOSITAE

\section{Vernonia}

I376r angustifolia Michx. I3762 pumila Chapm.

I 3763 Texana T. \& G.
I3764 Baldwinii Torr.

I 3765 crinita Raf.

(V. Arkansana DC.)

I3766 Drummondii Shuttlw.

( $V$. altissima var. grandiflora)

I 3767 fasciculata Michx.

I 3768 flaccidifolia Small

I 3769 gigantea (Walt.) Britton

( $V$. altissima Nutt.)

13770 glauca (L. Britton

( $V$. Noveboracensis var. latifolia)

I377I interior Small

I 3772 Lettermani Engelm.

I 3773 Lindheimeri Engelm \& Gray

I 3774 marginata (Torr.) Britton ( V. Jamesii T. \& G.)

I3775 maxima Small

I 3776 Noveboracensis (L.) Willd

I3777 oligophylla Michx.

I 3778 pulchella Small

I3779 scaberrima Nutt.

I 3780 tenuifolia Small.

I378I tomentosa (Walt.) Ell.

Stokesia

I 3782 laevis (Hill) Greene (S. cyanea I'Her.)

\section{Elephantopus}

I 3783 Carolinianus Willd.

I3784 nudatus A. Gray

I 3785 tomentosus L.

\section{Hartwrightia}

I 3786 Floridana A. Gray

\section{Sclerolepis}

I 3787 uniflora (Walt.) Porter (S. verticillata Cass.)

\section{Ageratum}

I 3788 conyzoides L.

I3789 corymbosum Zucc.

I 3790 littorale A. Gray

\section{Stevia}

r379I amabilis Lemmon

13792 Lemmoni A. Gray

I 3793 micrantha Lag.

I 3794 Plummerae A. Gray

I3795 alba A. Gray 

I3796 salicifolia Cav.

I3797 serrata Cav.

\section{Hofmeisteria}

13798 pluriseta A. Gray

\section{Trichocoronis}

I3799 riparia Greene

I380o rivularis A. Gray

I 380 I Wrightii A. Gray

\section{Carminatia}

I3802 tenuiflora DC.

\section{Eupatorium}

I 3803 ageratifolium DC.

I 3804 acuminatum Coult.

r 3805 ageratoides L. f.

I3806 angustatum A. Gray

r 3807 album L.

I 3808 subvenosum A. Gray

I 3809 altissimum L.

I381o amoenum Pursh

I38II anomalum Nash

I3 8 I 2 aromaticum L.

I 38 I 3 incisum A. Gray

I38I4 melissoides A. Gray

I38I5 betonicum Hemsl.

I38I6 subintegrum A. Gray

I 38 I 7 Bigelovii A. Gray

I 38 I 8 Bruneri A. Gray

I38I9 capillifolium (Lam.) Small (E. foeniculaceum Willd.)

I 3820 coelestinum L.

I 382 I compositifolium Walt.

I 3822 conyzoides Vahl

I 3823 coronopifolium Willd.

I 3824 cuneifolium Willd.

I 3825 falcatum Michx.

(E. purpureum var. angustifolium)

I3826 Fendleri A. Gray

I3827. Greggii A. Gray

I $3^{828}$ heteroclinium Griseb.

r 3829 hyssopifolium L.

I 3830 angustissimum Mohr.

I38 3 incarnatum Walt.

I 3832 incisum Nutt.

I 3833 ivaefolium L.

I 3834 jucundum Greene

I3835 lecheaefolium Greene
I 3836 leptophyllum DC.

I 3837 leucolepis 'T. \& G.

I 3838 linearifolium Walt.

I 3839 maculatum L.

I 3840 mikanioides Chapm.

I 384 I occidentale Hook.

23842 Arizonicum A. Gray

I 3843 pauperculum A. Gray

I 3844 perfoliatum L.

I 3845 cuneatum Engelm.

I 3846 truncatum (Muhl.)A.Gray

I 3847 petalodium Britton

I3848 pinnatifidum El1.

I 3849 pubescens Muhl.

(E. rotundifolium var. ovatum Torr.)

I 3850 purpureum L.

I 3851 pycnocephalum Less.

I 3852 resinosum Torr.

I 3853 Rothrockii A. Gray

I3854 rotundifolium L.

I3855 scabridum A. Gray

I 3856 sagittatum A. Gray

I 3857 . semiserratum DC.

I $385^{8}$ lancifolium A. Gray

I 3859 serotinum Michx.

I3860 sessilifolium L.

I386I Brittonianum Porter

I 3862 solidaginifolium A. Gray

I3863 Torreyanum Short

(E. hyssopifolium var. laciniatum)

I 3864 Vaseyi Porter

I 3865 verbenaefolium Michx. (E. teucrifolium Willd.)

I 3866 villosum Sw.

I 3867 Wrightii A. Gray

\section{Willughbaea}

(Mikania)

I 3868 cordifolia (L.) Kuntze

I3869 scandens (L.) Kuntze

\section{Trilisa}

I 3870 odoratissima (Walt.) Cass.

I 387 I paniculata (Walt.) Cass.

Carphochaeta

I 3872 Bigelovii A. Gray 



\section{Coleosanthus}

(Brickellia)

I3873 abbreviatus (A. Gray) Greene I 3874 ambigens Greene

I 3875 atractyloides(A.Gray)Kuntze I 3876 baccharideus(A.Gray)Kuntze I 3877 betonicifolius (A. Gray)

Kuntze

I 3878 brachyphyllus (A. Gray)

Kuntze

I 3879 Californicus ('T.\& G.) Kuntze I3880 Cedrosensis Greene

I388I cordifolius (E11.) Kuntze

I 3882 Coulteri (A. Gray) Kuntze

I3883 cylindraceus(Engelm.\&Gray)

I3884 dentatus (DC.) Kuntze

Kuntze

(B. Riddellii A. Gray)

I 3885 desertorum Coville

I 3886 floribundus (A. Gray) Kuntze I 3887 frutescens (A. Gray) Kuntze I 3888 grandiflorus (Hook.) Kuntze I 3889 Greenei (A. Gray) Kuntze r3890 humilis Greene

r389I incanus (A. Gray) Kuntze

I3892 Knappianus (Drew) Kuntze

I3893 laciniatus (A. Gray) Kuntze

I 3894 Lemmoni (A. Gray) Kuntze I3895 linifolius (D. C. Eaton)

Kuntze

r3896 longifolius (Wats.) Kuntze

I 3897 microphyllus (Nutt.) Kuntze

I 3898 Mohavensis (A. Gray) Kuntze

I 3899 multiflorus (Kellogg) Kuntze

I 3900 Nevinii (A. Gray) Heller

I 390 I oblongifolius (Nutt.) Kuntze

I3902 oliganthus (Less.) Kuntze

I 3903 parvulus (A. Gray) Kuntze

13904 petiolaris (A. Gray) Greene

I 3905 Pringlei (A. Gray) Kuntze

I3906 Rusbyi (A. Gray) Kuntze

I 3907 scaber Greene

I 3908 simplex (A. Gray) Kuntze

I 3909 squamulosus (A.Gray)Kuntze

I39I0 Wislizeni (A. Gray) Kuntze

I39I I Wootoni Greene

I 3912 Wrightii (A. Gray) Britton

\section{Kuhnia}

I 39 I 3 enpatorioides L.

I39r4 glutinosa Ell.

(K. eupatorioides var. corymbulosa)

I39I 5 Kuhnia (Gaertn.) Mohr

I39I6 rosmarinifolia Vent.

\section{Lacinaria}

(Liatris)

I 39 I 7 acidota (Engelm. \& Gray)

Kuntze

I 3918 Boykini ('T. \& G.) Kuntze

I3919 Chapmani ('T. \& G.) Kuntze

I3920 longifolia Nash

I 392 I cylindracea (Michx.) Kuntze

I3922 cymosa Ness

I 3923 elegans (Walt.) Kunt'e

I3924 flabellata Small

I 3925 Garberi (A. Gray) Kunt'e

I 3926 gracilis (Pursh) Kunt'e

I3927 graminifolia (Walt.) Kunt'e

I3928 Halei Small

I 3929 Helleri Porter

I3930 lancifolia Greene

r393 I laxa Small

I3932 microcephala Small

I3933 pauciflora (Pursh) Kunt'e

I 3934 pilosa (Ait.) Heller

I3935 platylepis Small

I3936 punctata (Hook.) Kunt'e

I 3937 pycnostachya(Michx.)Kunt'e

r 3938 Regimontis Small

I3939 scariosa (L.) Hill

I 3940 corymbulosa Sheldon

I 394I squarrulosa (Michx)Sinall

I3942 spicata (L.) Kunt'e

I 3943 pumila (Lodd.) Porter

I 3944 squarrosa (L.) Hill

I 3945 intermedia (Lindl.) Porter

r 3946 tenuifolia (Nutt.) Kunt'e

\section{Garberia}

I 3947 fruticosa (Nutt.) A. Gray

\section{Carphephorus}

I 3948 bellidifolius (Michx.) T. \& G.

I 3949 corymbosus (Nutt.) T. \& G.

I 3950 Pseudo-Liatris Cass.

I 395 I tomentosus (Michx.) T. \& G. 



\section{Gymnosperma}

I 3952 corymbosa DC.

\section{Xanthocephalum}

I 3953 Californicum (DC.) Greene (Gutierrezia Californica T. \& G.) I 3954 gymnospermoides (A. Gray)

I3955 Wrightii A. Gray B. \& H.

\section{Grindelia}

I 3956 Arizonica A. Gray

13957 Brownii Heller

I3958 camporum Greene

I3959 cuneifolia Nutt.

r 3960 decumbens Greene

I396r decurrens Greene

I3962 fastigiata Greene

I3963 grandiflora Hook.

I3964 Hẹndersoni Greene

I 3965 hirsuta H. \& A.

I 3966 humilis H. \& A.

r 3967 inornata Greene

I3968 integrifolia DC.

I3969 inuloides Willd.

I 3970 microcephala (DC.)

I397 I lanata Greene

I 3972 lanceolata Nutt.

I 3973 latifolia Kellogg

I 3974 macrophylla Greene

I 3975 nana Nutt.

I3976 discoidea A. Gray

I3977 Oregana A. Gray

I 3978 Pacifica $_{\text {" Jones }}$

I 3979 paludosa Greene

I 3980 patens Greene

I 398 I perennis Aven Nelson

I 3982 platylepis Greene

I 3983 procera Greene

I3984 robusta Nutt.

I 3985 platyphylla Greene

I 3986 rubricaulis DC.

I 3987 maritima Greene

I 3988 scabra Greene

I 3989 squarrosa (Pursh) Dunal

I 3990 nuda (Wood) A. Gray

I399I subalpina Greene

\section{Vanclevea}

I 3992 stylosa (Eastwood) Greene

(Grindelia stylosa Eastwood)

\section{Gutierrezia}

I 3993 Californica (DC.) 'T. \& G.

I 3994 eriocarpa A. Gray

I 3995 lucida Greene

I 3996 microcephala (DC.) A. Gray

I 3997 Sarothrae (Pursh) B. \& R.

(G. Euthamiae T. \& G.)

I 3998 sphaerocephala A. Gray

I 3999 Texana T. \& G.

\section{Amphiachyris}

I 4000 dracunculoides (DC.) Nutt.

I 400 I Fremontii A. Gray

\section{Pentachaeta}

I4002 alsinoides Greene

I4003 aphantochaeta Greene

I 4004 anrea Nutt.

I 4005 bellidiflora Greene

I4006 exilis A. Gray

I4007 Lyoni A. Gray

\section{Xanthisma}

I 4008 Texanum DC.

\section{Acamptopappus}

I4009 Shockleyi A. Gray

I 40 Io sphaerocephalus A. Gray

\section{Mauchia}

(Bradburya)

I 40 I I hirtella (T. \& G.) Kuntze

\section{Heterotheca}

I4OI 2 grandiflora Nutt.

I 4 OI 3 subaxillaris (Lam.) B. \& R. (H. Lamarckii Cass.)

\section{Chrysopsis}

I 40I 4 argentea (Pers.) Ell.

' I40r5 Bakeri Greene

I40I6 Berlandieri Greene

14017 Bolanderi A. Gray

I 4018 Breweri A. Gray

I40I9 caespitosa Jones

I 4020 canescens (DC.) 'T. \& G.

I 402 I camporum Greene 

I4022 Columbiana Greene I 4023 depressa Rydb. 14024 echioides Benth. I 4025 falcata (Pursh) Ell. I4026 fastigiata Greene I 4027 foliosa Nutt.

I 4028 flexuosa Nash I 4029 floribunda Greene I 4030 fulcrata Greene I4O3I graminifolia (Michx.) Nutt. 14032 aspera A. Gray I4033 latifolia Fernald I 4034 hirsuta Greene I 4035 hirsutissima Greene I 4036 hispida (Hook.) Nutt. I4037 hyssopifolia Nutt. I 4038 latisquamea Pollard I 4039 Mariana (L.) Nutt. I 4040 mollis Nutt.

I 404I nivea Greene

I 4042 Nuttallii Britton (C. pilosa Nutt.)

I 4043 oligantha Chapm. I 4044 Oregana (Nutt.) A. Gray I 4045 scaberrima A. Gray r4046 pedunculata Greene I 4047 pilosa (Walt.) Britton (C. gossypina Nutt.)

I4048 pinifolia Ell.

I 4049 pumila Greene

I4050 rudis Greene

I405I Ruthii Small

I 4052 Rutteri (Rothrock) Greene I 4053 scabrella T. \& G. I 4054 sessiliflora Nutt.

I 4055 stenophylla (A. Gray) Greene I 4056 trichophylla (Nutt.) Ell. I 4057 villosa (Pursh) Nutt. $1405^{8}$ discoidea A. Gray I 4059 viscida (A. Gray) Greene I 4060 Wrightii A. Gray

\section{Solidago}

I406I alpestris Wald. \& Kit. I4062 amplexicaulis T. \& G. I 4063 arguta Ait.

I 4064 bicolor L. I 4065 Bigelovii A. Gray I4066 Wrightii A. Gray
I 4067 Boottii Hook.

I 4068 Ludoviciana A. Gray

I 4069 brachyphylla Chapm.

I 4070 Buckleyi T. \& G.

I 407 I caesia L.

14072 axillaris (Pursh) A. Gray

I 4073 paniculata A. Gray

I4074 Californica Nutt.

I 4075 Nevadensis A. Gray

I 4076 Canadensis L.

I4077 Arizonica A. Gray

14078 canescens A. Gray

I4079 gilvocanescens Rydb.

I4080 glabrata Porter

I 408I scabriuscula Porter

I4082 Chapmani A. Gray

I 4083 Chrysopsis Small

I4084 ciliosa Greene

r 4085 concinna Aven Nelson

I 4086 confertiflora DC.

I 4087 confinis A. Gray

I 4088 corymbosa Ell.

I 4089 Curtisii T. \& G.

I 4090 decumbens Greene

I 409I delicatula Small

I 4092 dilatata Aven Nelson

I4093 dispersa Small

14094 Drummondii T. \& G.

I4095 Elliottii 'T. \& G.

I4096 elongata Nutt.

I 4097 erecta Pursh

I 4098 fasciculata Greene

I4099 fistulosa Mill.

(S. pilosa Walt.)

I4IOO flaccidifolia Small

I 4IOI flavovirens Chapm.

I 4102 flexicaule L.

\section{(S. latifolia L.)}

I4IO3 Gattingeri Chapm.

I4ro4 glomerata Michx.

I 4105 gracillima T. \& G.

I4I06 Guiradonis A. Gray

I4ro7 Helleri Small

I4I08 hispida Muhl.

(S. bicolor var. concolor T. \& G.)

I4IO9 Houghtoni T. \& G.

r4I Io juncea Ait.

I4III ramosa Porter \& Britton 

I4I I 2 scabrella ('T.\&G.) A. Gray I4II3 lanata Hook.

I4II4 lancifolia T. \& G.

I4II5 Leavenworthii T. \& G.

I4II6 lepida DC.

I 4 I 7 Lindheimeriana Scheele

I4II8 macrophylla Pursh

I4II9 Marshallii Rothrock

I 4120 Missouriensis Nutt.

I4I I extraria A. Gray

I4I22 fasciculata Holzinger

I4123 montana A. Gray

I 4124 mollis Bartl.

(S. nemoralis var. incana A. Gray)

I 4I 25 monticola T. \& G.

I4I 26 inultiradiata Ait.

I4I27 Neo-Mexicana A. Gray

I 4I28 scopulorum A. Gray

I 4 I 29 nana Nutt

I4I30 Nashii Small

I 4I 3 neglecta T. \& G.

I 4132 nemoralis Ait.

I4I33 arenicola Burgess

I4I 34 nitida T. \& G.

I 4 I 35 odora Ait.

I4I 36 inodora A. Gray

I4I 37 Ohionis Riddell

I 4 I 38 oreophila Rydb.

I4I39 Parryi (A. Gray) Greene (Aplopappus Parryi A. Gray)

I4I40 minor (A. Gray) Greene

I4I 4 I patula Muhl.

I 4 I 42 strictula T. \& G.

I 4 I 43 petiolaris Ait.

I4r 44 plumosa Small

I4I45 procera Ait.

I 4 I 46 pubens M. A. Curtis

I 4 I 47 puberula Nutt.

I 4 I48 pulcherrima Aven Nelson

I4I49 pulverulenta Nutt.

14I50 Purshii Porter

(S. humilis Pursh)

I4I 5 I racemosa Greene

I 4 I 52 radula Nutt.

I4I53 Riddellii Frank

I 4 I 54 rigida L.

I4I 55 rigidiuscula ('T. \& G.) Porter

I4 56 Roanensis Porter
I4I57 rugosa Mill.

I4I $5^{8}$ rupestris Raf.

I 4 I59 sempervirens I.

I4160 viminea (Ait.) A. Gray

I4I6I serotina Ait.

I 4162 gigantea (Ait.) A. Gray

${ }_{14163}$ Shortii T. \& G.

I4I64 sparsiflora A. Gray

14165 subcinerea A. Gray

I 4166 spathulata DC.

I4I67 speciosa Nutt.

I 4168 pallida Porter

I4I69 spectabilis (D. C. Eaton)

I4I70 spithamaea M. A. Curtis

I4I7I squartosa Muhl.

14172 stricta Ait.

I 4 I 73 angustifolia (E11.) A.Gray

I 4 I 74 Terrae-Novae 'T. \& G.

I 4 I 75 trinervata Greene

I4176 Tolmieana A. Gray

I 4 I 77 tortifolia Ell.

I41 78 uliginosa Nutt.

I4I79 ulmifolia Muhl.

I4I80 microphylla A. Gray

I4I8I uniligulata (DC.) Porter

I4I 82 Vaseyi Heller

I 4183 verna $M$. A. Curtis

I4I 84 Virgaurea L.

I 4 I 85 Deanei Porter

I 4 I 86

14187

I 4188

Gillmani (A. Gray) Porter

Randii Porter

I 4 I 89 Yadkinensis (Porter) Small

\section{Brintonia}

I4I9o discoidea (E11.) Greene

(Solidago discoidea Ell.)

\section{Euthamia}

(Solidago in part)

I4I9I Caroliniana (L.) Greene

(S. tenuifolia Pursh)

I 4192 graminifolia (L.) Nutt.

(S. lanceolata L.)

I4193 leptocephala (T.\& G.) Greene

I 4194 occidentalis Nutt. 



\section{Brachychaeta}

I4I95 sphacelata (Raf.) Britton (B. cordata T. \& G.)

\section{Petradoria}

I4I96 pumila (Nutt.) Greene (Solidago pumila T. \& G.)

\section{Chrysoma}

(Aplopappus mainly)

14197 arborescens (A. Gray) Greene (Bigelovia arborescens A. Gray) I4I98 brachylepis (A. Gray) Greene

(Bigelovia brachylepis A. Gray) I4199 Cooperi (A. Gray) Greene

(Bigelovia Cooperi A. Gray) r 4200 cuneata (A. Gray) Greene I420I spathulata(A.Gray)Greene 14202 ericoides (Less.) Greene I 4203 laricifolia (A. Gray) Greene I4204 nana (Nutt.) Greene I4205 Palmeri (A. Gray) Greene I4206 paniculata (A. Gray) Greene (Bigelovia paniculata A. Gray) I 4207 Parishii Greene

I4208 pauciflosculosa (Michx.)

Greene

(Solidago panciflosculosa Michx.) I 4209 pinifolia (A. Gray) Greene I42IO teretifolia (Dur. \& Hilg.)

Greene

(Bigelovia teretifolia A. Gray)

Prionopsis

I42I I ciliata Nutt.

(Aplopappus ciliatus DC.)

\section{Pyrrocoma}

(Aplopappus in part)

I 42 I 2 apargioides (A. Gray) Greene I 42 I 3 arguta (Nutt.) T. \& G. 142 I 4 carthamoides Hook.

I42 I 5 ciliolata Greene

I 4216 congesta Greene

I42I 7 crocea (A. Gray) Greene

I421 8 Cusickii (A. Gray) Greene

I42I9 elata Greene

I 4220 eriopoda Greene

I422I genuflexa Greene

I4222 glomerata (Nutt.) T. \& G.
I4223 gossypina Greene

I 4224 hirta (A. Gray) Greene

I 4225 Howellii (A. Gray) Greene

I4226 insecticruris (Henderson)

Heller

I 4227 integrifolia (Porter) Greene

14228 inuloides (Nutt.) Greene

I 4229 lanceolata (Hook.) Greene

I4230 longifolia Greene

I423I Lyallii (A. Gray) Rydb. I 4232 paniculata (Nutt.) Torr.

I4233 stenocephala (A. Gray)

Greene

I 4234 virgata (A. Gray) Greene

I 4235 racemosa (Nutt.) T. \& G.

I 4236 radiata Nutt.

I4237 solidaginea Greene

r4238 subsquarrosa Greene

I 4239 subviscosa Greene

24240 tenuicaulis (D. C. Eaton)

Greene

I424I uniflora (Hook.) Greene

\section{Oonopsis}

(Aplopappus in part)

14242 argillacea Aven Nelson

I 4243 Engelmanni (A.Gray) Greene

(Bigelovia Engelmanni A. Gray)

I 4244 foliosa (A. Gray) Greene

(A. Fremonti A. Gray)

I4245 multicaulis (Nutt.) Greene

I4246 Wardi (A. Gray) Greene

I4247 condensata Aven Nelson

\section{Eriocarpum}

(Aplopappus in part)

I 4248 australe Greene

I 4249 Coloradoense (A.Gray)Greene

(Aster Coloradoenisis A. Gray)

I4250 gracilis (Nutt.) Greene

I425I grindelioides Nutt.

(A. Nuttallii DC.)

I 4252 gymnocephalum (DC.)Greene

(Aster gymnocephalus DC.)

14253 junceum Greene

14254 megacephalum Nash

r 4255 phyllocephalum (DC.) Greene I 4256 rubiginosum (DC.) Britton 

I4257 serratum Greene

$1425^{8}$ spinulosum (Pursh) Greene I4259 Wootoni Greene

\section{Isopappus}

14260 divaricatus (Nutt.) T. \& G.

\section{Stenotus}

(Aplopappus in part)

I426I acaulis Nutt.

I4262 armerioides Nutt.

r 4263 caespitosus Nutt.

I4264 interior (Coville) Greene

I 4265 laceratus (Henderson) Heller I 4266 lanuginosus (A. Gray) Greene 14267 linearifolius (DC.) T. \& G.

14268 MacLeanii (T. S. Brandegee)

Heller

I4269 stenophyllus (A.Gray) Greene

\section{Macronema}

(Aplopappus in part)

14270 discoideum Nutt.

I427 I Greenei (A. Gray) Greene

14272 grindelifolium Rydb.

I4273 lineare Rydb.

I4274 molle (A. Gray) Greene

I4275 pygmaeum ('T. \& G.) Greene

I 4276 suffruticosum Nutt.

14277 Watsoni (A. Gray) Greene

\section{Osbertia}

I4278 Heleniastrum Greene

I4279 glabratus Greene

\section{Eastwoodia}

I4280 elegans Brandegee

\section{Chrysothamnus}

(Aplopappus and Bigelovia)

I428I affinis Aven Nelson

I4282 attennatus (Jones)

Aven Nelson

I 4283 albidus (Jones) Greene

14284 Arizonicus. Greene

I4285 Bakeri Greene

I4286 Bigelovii (A. Gray) Greene I 4287 Bloomeri (A. Gray) Greene I4288 Bolanderi (A. Gray) Greene I4289 Californicus (A. Gray) Greene
I4290 ceruminosus (Dur. \& Hilg.)

1429I collinus Greene Greene

I4292 depressus Nutt.

14293 elegans Greene

I 4294 formosus Greene

I4295 frigidus Greene

I4296 concolor Aven Nelson

I4297 glaucus Aven Nelson

r4298 gnaphalodes Greene

I 4299 graveolens (Nutt.) Greene

(B. graveolens var. glabrata Gray)

I4300 Greenei (A. Gray) Greene

I430I Howardi (Parry) Greene

14302 humilis Greene

I 4303 junceus Greene

I 4304 lanceolatus Nutt.

I4305 latisquameus (A.Gray)Greene

I4306 leiospermus (A. Gray) Greene

I4307 linifolius Greene

I4308 Mohavensis Greene

I4309 nanus (Nutt.) Howell

I43IO nauseosus (Pursh) Brition

(B. graveolens A. Gray)

I43I I albicaulis (Nutt.) Rydb.

I43I 2 Nevadensis (A. Gray) Greene

r43 I 3 occidentalis Greene

I43I 4 oreophilus Aven Nelson

I43I5 pallidus Aven Nelson

I43I 6 Parryi (A. Gray) Greene

I43I7 Plattensis Greene

I 43 I 8 puberulus(D.C. Eaton)Greene

I4319 pulchellus (A. Gray) Greene

I 4320 pulcherrimus Aven Nelson

I432I fasciculatus Aven Nelson

I 4322 pumilus Nutt.

I4323 acuminatus Aven Nelson

I4324 rarus Aven Nelson

I 4325 resinosus (Nutt.) Howell

I4326 stenophyllus (A.Gray) Greene

I4327 tortifolius (A. Gray) Greene

I4328 Vaseyi (A. Gray) Greene

I4329 viscidiflorus (Hook.) Nutt.

(B. Douglasii A. Gray)

I4330 latifolius (D. C. Eaton)

Greene

I433I serrulatus ('Torr.) Greene I4332 Wyomingensis Aven Nelson 



\section{Chondrophora}

(Bigelovia in part)

I4333 nudata (Michx.) Britton

I4334 virgata (Nutt.) Greene

\section{Isocoma}

(Bigelovia in part)

14335 acradenia Greene

I4336 arguta Greene

14337 coronopifolia (A.Gray)Greene

I 4338 Drummondii (T.\&G.) Greene

I4339 Hartwegi (A. Gray) Greene

I 4340 heterophylla (A.Gray) Greene (B. Wrightii A. Gray)

I434I hirtella (A. Gray) Heller

I4342 pluriflora ('T. \& G.) Greene

I4343 tridentata Greene

I4344 veneta (H. B. K.) Greene

I4345 vernonioides Nutt.

\section{Hazardia}

(Aplopappus in part)

I 4346 cana (A. Gray) Greene

I4347 detonsa Greene

I 4348 obtusa Greene

I 4349 serrata Greene

I $435^{\circ}$ squarrosa (H. \& A.) Greene

I 435 I Whitneya (A. Gray) Greene

\section{Lessingia}

I $435^{2}$ adenophora Greene

r 4353 Germanorum Cham.

I4354 glandulifera A. Gray

I 4355 hololeuca Greene

I 4356 Lemmoni A. Gray

I 4357 leptoclada A. Gray

I $435^{8}$ microcephala A. Gray

I 4359 nana A. Gray

I 4360 caulescens A. Gray

I436I nemaclada Greene

I4362 Parryi Greene

I4363 parvula Greene

14364 pectinata Greene

14365 ramulosa A. Gray

I 4366 tenuis (A. Gray) Coville

I 4367 virgata A. Gray

\section{Bourdonia}

(Keerlia)

TGreene
14369 effusa (A. Gray) Greene

\section{Aphanostephus}

I4370 Arizonicus A. Gray

I437 I humilis (Benth.) A. Gray

14372 ramosissimus DC.

I4373 skirrobasis (DC.) Trelease (A. Arkansanus A. Gray)

Greenella

I4374 Arizonica A. Gray

r 4375 discoidea A. Gray

\section{Thurovia}

I 4376 triflora Rose

\section{Bellis}

I4377 integrifolia Michx.

I4378 perennis L.

\section{Psilactis}

I4379 asteroides A. Gray

14380 Coulteri A. Gray

\section{Chaetopappa}

I 438I asteroides DC.

$\mathrm{I} 43^{82}$ imberbis A. Gray

I 4383 modesta (DC.) A. Gray

I 4384 Parryi A. Gray

\section{Monoptilon}

I 4385 bellidiforme T. \& G.

\section{Dichaetophora}

I4386 campestris A. Gray

\section{Boltonia}

I4387 asteroides (L.) L'Her.

I 4388 decurrens (T. \& G.) Wood

I 4389 diffusa Eill.

I 4390 latisquamea A. Gray

r439I occidentalis (A. Gray) Howell

\section{Townsendia}

I4392 alpigena Henderson

14393 alpina A. Gray

I4394 Arizonica A. Gray

I4395 Bakeri Greene

I 4396 condensata Parry

I4397 eximia A. Gray

I 4398 exscapa (Richards.) Porter

(T. sericea Hook.)

r4399 Fendleri A. Gray 

I4400 florifer (Hook.) A. Gray

I 4401 communis Jones

I 4402 glabella A. Gray

14403 grandiflora Nutt.

I4404 incana Nutt.

I 4405 Parryi D. C. Eaton

I 4406 Rothrockii A. Gray

14407 scapigera D. C. Eaton

I 4408 ambigua A. Gray

I4409 caulescens D. C. Eaton

I44IO spathulata Hook.

I 44 I I strigosa Nutt.

I44I 2 Watsoni A. Gray

I44I3 Wilcoxiana Wood

\section{Corethrogyne}

14414 caespitosa Greene

I 44I 5 Californica DC.

I44I6 filaginifolia (H. \& A.) Nutt.

I44I 7 rigida A. Gray

I 44I 8 robusta Greene

I 44I9 obovata Benth.

r 4420 virgata Benth.

r442I viscidula Greene

\section{Eremiastrum}

I 4422 bellioides A. Gray

I 4423 Orcuttii Wats.

\section{Aster}

I4424 acuminatus Michx.

14425 adnatus Nutt.

14426 adscendens Lindl.

I 4427 denudatus T. \& G.

I 4428 alpinus L.

I4429 amethystinus Nutt.

14430 amplexifolius Rydb.

I443 I amplissimus Greene

I 4432 amplus Lindl.

I4433 andinus Nutt.

r4434 anomalus Engelm.

I 4435 apricus (A. Gray) Rydb.

I 4436 arenarioides D. C. Eaton

I 4437 azureus Lindl.

I $443^{8}$ blepharophyllus A. Gray

14439 Boltoniae Greene

I 4440 borealis Provancher

I 444 I brevibracteatus Rydb.

14442 Burkei (A. Gray) Howell
I 4443 campestris Nutt.

I4444 Bloomeri A. Gray

I 4445 Camptosorus Small

14446 carmesinus Burgess

I 4447 Carolinianus Walt.

I 4448 Chapmani T. \& G.

I 4449 Chilensis Nees

(A. Chamissonis A. Gray)

I 4450 ciliomarginatus Rydb.

I445I Claytoni Burgess

I 4452 crispicans Burgess

I 4453 concinnus Willd.

I 4454 concolor L.

I 4455 conspicuus Lindl.

14456 continuus Small

I 4457 Cordelanus Henderson

I 4458 cordifolius $\mathrm{L}$.

I44.59 alvearius Burgess

I4460 Furbishiae Fernald

I 446I pedicellatus Burgess

I 4462 polycephalus Porter

I 4463 Curtisii T. \& G.

r 4464 curvescens Burgess

r 4465 oviformis Burgess

I 4466 umbelliformis Burgess

I 4467 Cusickii A. Gray

I 4468 Lyallii A. Gray

I 4469 denudatus Nutt.

14470 divaricatus L.

(A. corymbosus Ait.)

I 447 I curtifolius Burgess

I 4472 cymulosus Burgess

I4473 deltoideus Burgess

I4474 fontinalis Burgess

I 4475 persaliens Burgess

I4476 Douglasii Lindl.

I 4477 Drummondii Lindl.

14478 dumosus L.

I4479 coridifolius (Michx.)

14480

I 448 I

I4482 Eatoni (A. Gray) Howell

I 4483 Elliottii T. \& G.

I 4484 ericoides L.

I 4485

I 4486

I 4487 depauperatus Porter pilosus (Willd.) Porter platyphyllus T. \& G. 

I4488 parviceps Burgess

I 4489 Randi Britton

I 4490 exilis Ell.

I 449 I australis A. Gray

I 4492 falcatus Lindl.

I 4493 Faxoni Porter

(A. polyphyllus Willd.)

I4494 Fendleri A. Gray

I 4495 foliaceus Lindl.

14496 Canbyi A. Gray

I 4497 Forwoodii Wats.

14498 Fremontii (T. \& G.) A. Gray

I 4499 Parishii A. Gray

I 4500 frondeus (A. Gray) Greene

I450r furcatus Burgess

I 4502 Geyeri (A. Gray) Howell

I 4503 glomeratus (Nees) Bernh.

I4504 gracilis Nutt.

I4505 grandiflorus L.

I 4506 Hallii A. Gray

I 4507 Hendersoni Fernald

I4508 Herveyi A. Gray

I 4509 hesperius A. Gray

I45 IO Wootoni Greene

I45 I I hirsuticaulis Lindl.

I $45^{1} 2$ ianthinus Burgess

145 13 incanopilosus(Lindl.)Sheldon (A. commutatus A. Gray)

I 45 I 4 integrifolius Nutt.

I 45 I 5 invenustus Greene

I 45I6 Jessicae Piper

I $45^{1} 7$ junceus Ait.

I 4518 Kingii D. C. Eaton

I45I9 laevis $\mathrm{L}$.

I4520 amplifolius Porter

I 452 I Potomacensis Burgess

I 4522 lateriflorus (L.) Britton (A. diffusus Ait.)

14523 glomerellus (T. \& G.)

I 4524 grandis Porter [Burgess

I 4525 horizontalis(Desf.) Burgess

14526

I 4527 pendulus (Ait.) Burgess thyrsoideus (A. Gray)

I4528 Lemmoni A. Gray [Sheldon

I 4529 lentus Greene

14530 Lindleyanus T. \& G.

I 453 I ciliolatus A. Gray

$1453^{2}$ eximius Burgess
I 4533 longifolius Lam.

I 4534 villicaulis A. Gray

I 4535 Lowrieanus Porter

I4536 Bicknellii Porter

14537 lancifolius Porter

I $453^{8}$ macrophyllus L.

14539 apricensis Burgess

I 4540 biformis Burgess

I454I excelsior Burgess

I 4542 pinguifolius Burgess

I 4543 sejunctus Burgess

I4544 velutinus Burgess

I 4545 majus (Hook.) Porter (A. modestus Lindl.)

I4546 Menziesii Lindl.

I 4547 militaris Greene

I 4548 mirabilis T. \& G.

I4549 Missouriensis Britton

I $455^{\circ}$ multiflorus Ait.

I $455^{1}$ multiformis Burgess

I4552 Nebraskensis Britton

I 4553 nemoralis Ait.

I4554 Blakei Porter

I 4555 nobilis Burgess

I 4556 Nova-Angliae L.

r 4557 roseus (Desf.) DC.

I4558 Nova-Belgii L.

I4559 Atlanticus Burgess

r 4560 Brittoni Burgess

I 456I elodes (T. \& G.) A. Gray

I 4562 laevigatus (Lam.) A. Gray

${ }^{1} 4563$ litorens A. Gray

I 4564 thyrsiflorus A. Gray

I4565 oblongifolius Nutt.

I 4566 occidentalis (Nutt.) T. \& G.

I 4567 intermedius A. Gray

I 4568 scabriusculus A. Gray

I 4569 Oreganus Nutt.

14570 oxyphyllus Greene

I 457 I Palmeri A. Gray

I 4572 paniculatus Lain.

I4573 bellidiflorus (Willd.)

Burgess

simplex (Willd.) Burgess

I4575 patens Ait.

14576 gracilis Hook.

I 4577 patulus Lain.

I 4578 pauciflorus Nutt. 

14579 gracillimus A. Gray

I 4580 phlogifolius Muhl.

I 458 I phyllolepis T. \& $\mathbf{G}$.

I 4582 Porteri A. Gray

${ }_{1} 45^{8} 3$ prenanthoides Muhl.

I 4584 porrectifolius Porter

I 4585 Pringlei (A. Gray) Britton

14586 ptarmicoides (Nees) T. \& G.

14587 Georgianus A. Gray

I 4588 lutescens (Hook.) A. Gray

I 4589 puniceus L.

I 4590 firmus (Nees) T. \& G.

I 459 I lucidulus A. Gray

I 4592 purpuratus Nees

(A. virgatus Ell.)

I4593 pygmaeus Lindl.

I4594 racemosus E11.

14595 radula Ait.

14596 biflorus (Michx.) Porter

I4597 radulinus A. Gray

I4598 Richardsonii Spreng.

(A. Sibiricus A. Gray, not L.)

14599 giganteus Hook.

I 4600 riparius $H . B$. K.

I460I roscidus Burgess

I 4602 sagittaefolius Willd.

I 4603 dissitiflorus Burgess

I 4604 urophyllus(Lindl.)Burgess

I 4605 salicifolius Lam.

I 4606 caerulescens A. Gray

I4607 stenophyllus (Lindl.)

Burgess

I4608 subasper (Lindl.) A. Gray

14609 Schreberi Nees

I46Io Scribneri Rydb.

I 46 I I sericeus Vent.

I 46I 2 Shortii Hook.

I 46 I 3 Sonomensis Greene

I46I4 spectabilis Ait.

I 46 I 5 squarrosus Walt.

I46I6 subracemosus Rydb.

I46I7 subspathulatns Rydb.

(A. spathulatus Lindl.)

I46I 8 subulatus Michx.

146I9 surculosus Michx.

I 4620 tardiflorus L.

I462 I lancifolius Fernald

I 4622 tenebrosus Burgess
14623 tenuifolius L.

I 4624 thermalis Jones

14625 Torreyi Porter

I 4626 tradescanti L.

I 4627 turbinellus Lindl.

I 4628 undulatus L.

I4629 abruptifolius Burgess

I4630 diversifolius A. Gray

I463I longiformis Burgess

I 4632 torquatus Burgess

I4633 triangularis Burgess

I 4634 versicolor Willd.

I 4635 vimineus Lam.

I 4636 Columbianus Britton

I4637 foliolosus (Ait.) A. Gray

I 4638 violaris Burgess

I 4639 viridis Nees

I4640 Watsoni A. Gray

r 464I Woodhousei Wooton

I 4642 Yosemitanus (A.Gray)Greene

\section{Heleastrum}

(Aster in part)

I 4643 Chapmani ( $T$. \& G.) Greene (A. eryngiifolius T. \& $\mathrm{G}$.)

I 4644 paludosum (DC.) Greene

I4645 spinosum (Chapm.) Greene

\section{Doellingeria}

(Aster in part)

I4646 humilis (Willd.) Britton

I 4647 infirma (Michx.) Greene

I4648 reticulata (Pursh) Greene

I4649 sericocarpoides Small

I 4650 umbellata (Mill.) Nees

I 465I pubens (A. Gray) Britton

\section{Ionactis}

(Aster in part)

I 4652 alpina (Nutt.) Greene

(A. scopulorum A. Gray)

I4653 linariifolia (L.) Greene

I 4654 stenomeres (A. Gray) Greene

\section{Lencelene}

r 4655 arenosa Heller

I 4656 ericoides (Torr.) Greene

(A. ericaefolius Rothrock)

I4657 serotina Greene 



\section{Eucephalus}

(Aster in part)

I $465^{8}$ Covillei Greene

I4659 elegans Nutt.

I 4660 Engelmanni (A.Gray) Greene

I466I formosus Greene

I 4662 glabratus Greene

I 4663 glaucescens (A. Gray) Greene

I 4664 glaucus Nutt.

I 4665 ledophyllus (A. Gray) Greene

I4666 Macounii Greene

I 4667 nemoralis (Ait.) Greene

I 4668 paucicapitatus (Rob.) Greene I 4669 serrulatus Greene

\section{Xylorrhiza}

(Aster in part)

I 4670 frutescens (Wats.) Greene

I 467 I glabriuscula Nutt.

I 4672 Orcuttii(Vasey\&Rose)Greene

I 4673 Parryi (A. Gray) Greene

I 4674 tortifolia (T. \& G.) Greene

I 4675 venusta (Jones) Heller

I4676 villosa Nutt.

(A. Xylorrhiza T. \& G.)

I 4677 Wrightii (A. Gray) Greene

\section{Oreastrum}

(Aster in part)

I 4678 alpiginum ('T. \& G.) Greene I 4679 Andersoni (A. Gray) Greene I4680 elatum Greene

I468I Haydeni (Porter) Rydb.

(A. pulchellus D. C. Eaton)

\section{Leucosyris}

(Aster in part)

I 4682 carnosa (A. Gray) Greene I 4683 spinosa (Benth.) Greene

\section{Brachyactis}

(Aster in part)

I4684 angustus (Lind1.) Britton

I 4685 frondosus (Nutt.) A. Gray

\section{Machaeranthera}

(Aster in part)

I 4686 aspera Greene

I 4687 asteroides (Torr.) Greene
I 4688 attenuata Howell

(A. canescens var. latifolius A. Gray)

I4689 Bigelovii (A. Gray) Greene

I 4690 canescens (Pursh) A. Gray

I469I eradiata (A. Gray) Howell

I 4692 incana (Lindl.) Greene

I 4693 inornata Greene

I 4694 laetevirens Greene

I4695 leucanthemifolia Greene

I 4696 linearis Greene

I 4697 montana Greene

I4698 parviflora A. Gray

I4699 Pattersoni (A. Gray) Greene

I4700 sessiliflora (Nutt.) Greene

I470I Shastensis A. Gray

14702 superba Aven Nelson

I 4703 tanacetifolia (H. B. K.) Nees

\section{Erigeron}

I4704 accedens Greene

I4705 acris L.

I4706 Droebachensis(O.F.Muell)

I 4707 Aliceae Howell [Blytt

I 4708 alpinus L.

I4709 amplifolius Howell

I 47 IO angustatus Greene

I 47 I I angustifolius (A. Gray) Rydb.

I 4712 annus (L.) Pers.

I 47 I 3 aphanactus (A. Gray (Greene

I47I4 arenarius Greene

I 47 I 5 argentatus A. Gray

I 4716 Arizonicus A. Gray

I 47 I 7 asperugineus (D. C. Eaton)

I 4718 asper Nutt. (E. glabellus Nutt.)

I47I9 aureus Greene

(Aplopappus Brandegei A. Gray)

I4720 Anstinae Greene

I4721 barbellulatus Greene

I 4722 Bellidiastrum Nutt.

I 4723 Beyrichii (F. \& M.) T. \& G.

I4724 Bigelovii A. Gray

I47.25 Bioletti Greene

I 4726 Blasdalei Greene

r 4727 Blochmanae Greene

I 4728 Bloomeri A. Gray

I 4729 Bonariensis L.

I4730 Brandegei A. Gray 

I473I Breweri A. Gray

I 4732 caespitosus Nutt.

I4733 laccoliticus Jones

I 4734 nauseosus Jones

I 4735 Californicus Jepson

14736 calvus Coville

I 4737 canus A. Gray

I 4738 Cascadensis Heller

14739 cervinus Greene

I 4740 chrysopsidis A. Gray

I 474I brevifolius Piper

I 4742 cinereus A. Gray

I 4743 aridus Jones

I 4744 compositus Pursh

I 4745 trifidus A. Gray

I 4746 condensatus (D. C. Eaton)

I 4747 concinnus (H. \& A.) T. \& G.

I 4748 conflnis Howell

14749 consobrinus Greene

$1475^{\circ}$ conspicuus Rydb.

I 475 I corymbosus Nutt.

I4752 Coulteri Porter

I 4753 Covillei Greene

I4754 curvifolius Piper

I 4755 debilis (A. Gray) Rydb.

I 4756 decumbens Nutt.

14757 divergens T. \& G.

I $475^{8}$ Drummondii Greene

I 4759 Eatonii A. Gray

14760 elatior (A. Gray) Greene

I476I elatus (Hook.) Greene

r 4762 Bakeri Greene

14763 elegans Greene

I 4764 elegantulus Greene

I 4765 Elmeri Greene

r4766 Engelmanni Aven Nelson

I 4767 eriophyllus A. Gray

I 4768 eximius Greene

I 4769 filifolius Nutt.

I 4770 flabellifolius Rydb.

I 477 I flagellaris A. Gray

14772 foliosus Nutt.

14773 tenuissimus A. Gray

I 4774 formosissimus Greene

I4775 Forreri Greene

14776 frondeus Greene

I 4777 glandulosus Porter

I4799 Macounii Greene
14792 Lassenianus Greene

I 4778 glaucus Ker

I 4779 Gormani Greene

I4780 gracilis Rydb.

I 478I grandiflorus Hook.

I 4782 Grayi Heller (E. stenophyllus A. Gray)

I 4783 Hartwegi Greene

I 4784 Howellii A. Gray

I 4785 hyperboreus Greene

I 4786 hyssopifolius Michx

I 4787 inamoenus Aven Nelson

I 4788 inornatus A. Gray

r4789 jucundus Greene

I 4790 Kindbergii Greene

I479I lanatus Hook.

4793 leiomerus A. Gray

I 4794 Lemmoni A. Gray

I4795 leptophyllus Greene

14796 lonchophyllus Hook.

I 4797 luteus Aven Nelson

I 4798 MacDougalii Heller

I 4800 macranthus Nutt.

I 480 r melanocephalus Aven Nelson

14802 membranaceus Greene

I 4803 microlonchus Greene

I4804 minor (Hook.) Rydb.

(E. armeriaefolius of Syn. Flora)

14805 miser A. Gray

I 4806 modestus A. Gray

I4807 Montanensis Rydb.

(E. Tweedyana Canby \& Rose)

I4808 Muirii A. Gray

I 4809 multiceps Greene

I48Io multifidus Rydb.

I 48I I discoideus (A.Gray)Rydb.

I 48I 2 incertus Aven Nelson

I48I3 nudus Rydb.

I48I4 Nelsonii Greene

I48I 5 Neo-Mexicanus A. Gray

I 48 I6 Nevadensis A. Gray

I 4817 nudatus A. Gray

I48I 8 Nuttallii Heller

(E. foliosus var. stenophyllus Gray)

I48I9 oblanceolatus Rydb.

I 4820 ochrolencus Nutt.

I 482 I Oreganus A. Gray 

I4822 oxyphyllus Greene

14823 Pacificus Howell

I 4824 Parishii A. Gray

14825 Parryi Canby \& Rose

I 4826 peregrinus (Pursh) Greene (Aster peregrinus Pursh)

14827 Dawsoni Greene

14828 petrocallis Greene

I 4829 petrophilus Greene

I 4830 peucephyllus A. Gray

I 483I Philadelphicus L.

14832 pinnatisectus (A. Gray) Aven Nelson

I 4833 plantagineus Greene

14834 poliospermus A. Gray

14835 porphyreticus Jones

I 4836 Pringlei A. Gray

I 4837 pulchellus Michx.

(E. bellidifolius Muh1.)

I 4838 pumilus Nutt.

I 4839 purpuratus Greene

I 4840 pygmaeus (A. Gray) Greene I 484I quercifolius Lam.

I 4842 radicatus Hook.

14843 racemosus Nutt.

I 4844 ramosus (Walt.) B. S. P.

I 4845 repens A. Gray

I 4846 Robertianus Greene

I 4847 Rusbyi A. Gray

14848 salsuginosus (Rich.) A. Gray

I 4849 angustifolius A. Gray

I 4850 glacialis (Nutt.)A. Gray

I 485 I sanctarum Wats.

I 4852 Scribneri Canby

I4853 simplex Greene

I4854 Sonnei Greene

I4855 spathulifolius Rydb.

r 4856 speciosus (Lindl.) DC.

I 4857 stolonifer Greene

I4858 subcanescens Rydb.

I4859 subdecurrens Sch. Bip.

I 4860 subtrinervis Rydb.

I 486I supplex A. Gray

I 4862 tener A. Gray

14863 tenuis T. \& G.

I4864 tenuissimus Greene

I 4865 tetrapleuris (A. Gray) Heller (E. Utahensis A. Gray)
I 4866 trifidus Hook.

I 4867 Turneri Greene

I4868 Tweedyi Canby

I 4869 uniflorus L.

I 4870 ursinus D. C. Eaton

I 487 I vernus (L.) T. \& G.

I 4872 viscidula Greene

I 4873 Wyomingensis Aven Nelson

I 4874 Yellowstonensis Aven Nelson

\section{Wyomingia}

I 4875 cinerea Aven Nelson I 4876 pulcherrima (Heller)

\section{Leptilon}

Aven Nelson

(Evigeron in part)

I 4877 Canadense (L.) Britton

I4878 divaricatum (Michx.) Raf.

I4879 linifolium (Willd.)

\section{Sericocarpus}

I 4880 asteroides (L.) B. S. P.

(S. conyzoides Nees)

I488I bifoliatus (Walt.) Porter

(S. tortifolius Nees)

I 4882 Californicus Durand

14883 linifolius (L.) B. S. P.

(S. solidagineus Nees)

I 4884 Oregonensis Nutt.

I4885 rigidus Lindl.

\section{Conzya}

I4886 Coulteri A. Gray

\section{Baccharis}

I 4887 angustifolia Michx.

14888 Bigelovii A. Gray

I 4889 brachyphylla A. Gray

I489o consanguinea Greene

I489I Douglasii DC.

I4892 Emoryi A. Gray

I 4893 glomeruliflora Pers.

r 4894 glutinosa Pers.

I4895 halimifolia L.

I4896 Havardi A. Gray

I4897 neglecta Britton

I 4898 pilularis DC.

I 4899 Plummerae A. Gray

14900 pteronioides DC. 

I 490 I salicina T. \& G.

14902 sergiloides A. Gray

I 4903 Texana A. Gray

I 4904 thesioides H. B. K.

14905 viminea DC.

I 4906 Wrightii A. Gray

\section{Pluchea}

I 4907 camphorata (L.) DC.

I 4908 foetida (L.) B. S. P.

(P. bifrons $\mathrm{DC}$.)

I4909 imbricata (Kearney) Nash

I49Io longifolia Nash

r49I I petiolata Cass.

I 49I 2 sericea (Nutt.) Coville

\section{Pterocaulon}

I 4913 capitatum (H. \& A.) Britton I 49 I 4 pycnostachyum Ell.

I 4915 virgatum DC.

\section{Gnaphalodes}

(Micropus)

I 4916 amphibola (A. Gray) Greene I 4917 Californica (F. \& M.) Greene

\section{Stylocline}

I 491 8 Arizonica Coville

I49I9 gnaplialioides Nutt.

I 4920 micropoides A. Gray

\section{Ancistrocarphus}

I492I filagineus A. Gray

(Stylocline filaginea

\section{Psilocarphus}

I 4922 brevissimus Nutt.

I4923 elatior A. Gray

I 4924 globiferus Nutt.

I 4925 Oreganus Nutt.

I 4926 tenellus Nutt.

\section{Evax}

I 4927 candida ('T. \& G.) A. Gray I 4928 multicaulis DC.

I 4929 Drummondii ('T. \& G.) I 4930 prolifera Nutt.

\section{Hesperevax}

(Evax in part)

r 493 I acaulis (Kellogg) Greene
14932 brevifolia (A. Gray) Greene

I 4933 caulescens (Benth.) A. Gray

14934 humilis Greene

I 4935 sparsiflora (A. Gray) Greene

\section{Filago}

I 4936 Arizonica A. Gray

I 4937 Californica Nutt.

I $493^{8}$ depressa A. Gray

I4939 Gallica L.

I4940 Germanica L.

\section{Antennaria}

I494I aizoides Greene

14942 alpina (L.) Gaertn.

I 4943 alsinoides Greene

I 4944 ambigens (Greene) Fernald

I 4945 anaphaloides Rydb.

I4946 angustata Greene

I 4947 angustifolia Rydb.

I 4948 aprica Greene

I 4949 argentea Benth.

I 4950 arida Elias Nelson

I 495 I arnoglossa Greene

I 4952 borealis Greene

I 4953 bracteosa Rydb.

I 4954 Brainerdii Fernald

I 4955 calophylla Greene

I 4956 campestris Rydb.

I4957 Canadensis Greene

I $495^{8}$ Randii Fernald

I 4959 confinis Greene

A. Gray) I 4960 corymbosa Aven Nelson

I 496r decipiens Greene

I 4962 dimorpha (Nutt.) T. \& G.

I 4963 integra Henderson

I 4964 dioica (I.) Gaertn.

I 4965 exilis Greene

r 4966 fallax Greene

I 4967 Farwellii Greene

I 4968 flagellaris A. Gray

I 4969 flavescens Rydb.

I 4970 foliacea Greene

I497 I humilis Rydb.

I 4972 fusca Elias Nelson

14973 Geyeri A. Gray

I 4974 Holmii Greene

I 4975 Howellii Greene

I 4976 hyperborea (With.) Don 

14977 imbricata Elias Nelson

I 4978 insularis Greene

I4979 Labradorica Nutt.

I 4980 lanata (Hook.) Greene

I 498I luzuloides T. \& G.

I4982 Macounii Greene

I4983 marginata Greene

I 4984 media Greene

14985 microcephala A. Gray

I4986 microphylla Rydb.

I 4987 monocephala Greene

I 4988 mucronata Elias Nelson

I 4989 nardina Greene

I 4990 neglecta Greene

I 499 I subcorymbosa Fernald

I 4992 nemoralis Greene

I4993 neodioica Greene

I4994 attenuata Fernald

I4995 grandis Fernald

r4996 nitida Greene

I4997 oblanceolata Rydb.

I4998 oblancifolia Elias Nelson

I 4999 obovata Elias Nelson

I 5000 occidentalis Greene

I 500 P Parlinii Fernald

I 5002 parvifolia Nutt.

(A. rosea (D. C. Eaton) Greene)

r 5003 pedicellata Greene

I 5004 petaloidea Fernald

I 5005 scariosa Fernald

I 5006 Petasites Greene

I 5007 plantaginifolia (L.) Richards.

I 5008 petiolata (Fernald) Heller

I5009 propinqua Greene

I 50 Io pulcherrima (Hook.) Greene

I50I I pulvinata Greene

I5OI 2 racemosa Hook.

I5OI 3 recurva Greene

I 50 or 4 reflexa Elias Nelson

I5OI 5 rosulata $\mathrm{Rydb}$.

I 5016 rupicola Fernald

I 5017 scariosa Elias Nelson

I50I 8 solitaria Rydb.

r5or9 sordida Greene

I 5020 stenophylla A. Gray

I5O2I suffrutescens Greene

I 5022 umbrinella Rydb.

\section{Anaphalis}

I 5023 margaritacea (L.) B. \& H.

I 5024 occidentalis Greene

I 5025 subalpina (A. Gray) Rydb.

\section{Nacrea}

I5026 lanata Aven Nelson

\section{Gnaphalium}

I 5027 angustifolium Aven Nelson

I 5028 Arizonicum A. Gray

I 5029 bicolor Bioletti

I 5030 Californicum DC.

I 503I Chilense Spreng. (G. Sprengelii H. \& A.)

I5O32 confertifolium Greene

I 5033 decurrens Ives

r 5034 Helleri Britton

I 5035 lagopodioides Rydb.

I 5036 leucocephalum A. Gray

I 5037 microcephalum Nutt.

I 5038 Norvegicum Gunner

I 5039 obtusifolium L.

(G. polycephalum Michx.)

I 5040 palustre Nutt.

I 504 I purpureum L.

I 5042 ramosissimum Nutt.

I 5043 spathulatum Lam.

I5044 strictum A. Gray

I 5045 sulphnrescens Rydb.

I 5046 supinum L.

I 5047 sylvaticum L.

I 5048 thermale Elias Nelson

I 5049 uliginosum L.

I5050 Wrightii A. Gray

\section{Ereminula}

(Dimeresia)

I 505I Howellii (A. Gray) Greene

\section{Inula}

I5052 Helenium L.

\section{Adenocaulon}

I 5053 bicolor Hook.

\section{Polymuia}

I 5054 Canadensis L.

I 5055 radiata A. Gray

I 5056 laevigata Beadle

I 5057 Uvedalia L. 

Dicranocarpus

I $505^{8}$ parviflorus A. Gray

Guardiola

I 5059 platyphylla A. Gray

\section{Melampodium}

I 5060 cinereum DC.

I 506 I ramosissimum A. Gray

I 5062 hispidum H. B. K.

I 5063 longicornu A. Gray

I5064 perfoliatum H. B. K.

\section{Acanthospermum}

I5065 humile DC.

I 5066 xanthoides DC.

\section{Silphium}

I 5067 albiflorum A. Gray

I5068 asperrimum Hook.

I 5069 asteriscus L.

5070 angustatum A. Gray

I507 I laevicaule DC.

I5072 brachiatum Gattinger

I 5073 Chickamaugense Canby

I5074 collinum Greene

I5075 compositum Michx.

I 5076 gracile A. Gray

I5077 helianthoides Greene

I5O78 incisum Greene

I5079 integrifolium Michx.

I 5080 laciniatum L.

I508I Mohrii Small

I 5082 perfoliatum L.

I 5083 scaberrimum L.

I 5084 Simpsonii Greene

I 5085 terebinthinaceum Jacq.

I 5086 pinnatiflum(Ell.)A.Gray

I 5087 trifoliatum A. Gray

I5088 latifolium A. Gray

\section{Berlandiera}

I5089 lyrata Benth.

I509o macrophylla A. Gray

I 509 I subacaulis Nutt.

I 5092 Texana DC.

I5093 betonicifolia (Hook.)

I 5094 tomentosa (Pursh) Nutt.

T. \& G.

\section{Lindheimera}

I5095 T'exana Engelm. \& Gray
Chrysogonum

I 5096 Virginianum L.

I5097 dentatum A. Gray

Engelmannia

I 5098 pinnatifida T. \& G.

\section{Parthénice}

I 5099 mollis A. Gray

\section{Parthenium}

I.5100 alpinum T. \& G.

I5IOI argentatum A. Gray

I $5 \mathrm{IO2}$ auriculatum Britton

I $5^{103}$ Hysterophorus L.

I 5104 incanum H. B. K.

I 5105 integrifolium L.

I 5 I06 lyratum A. Gray

I 5107 repens Eggert

\section{Iva}

I 5108 ambrosiaefolia A. Gray

I5109 angustifolia Nutt.

I 5I IO axillaris Pursh

I5III pubescens A. Gray

I5II 2 caudata Small

I5II3 ciliata Willd.

I5II4 dealbata A. Gray

I5II5 frutescens L.

I5I 6 Hayesiana A. Gray

I 5 II 7 imbricata Walt.

I 5 II 8 microcephala Nutt.

I5II9 Nevadensis Jones

I 51 20 xanthifolia (Fresn.) Nutt.

\section{Oxytenia}

I5I2I acerosa Nutt.

\section{Dicoria}

I5122 Brandegei A. Gray

I 5 I23 canescens T. \& G.

\section{Hymenoclea}

I5I 24 monogyra T. \& G.

I 5125 Salsola T. \& G.

\section{Ambrosia}

15126 aptera DC.

I 5127 artemisiaefolia L.

I5128 bidentata Michx.

I5I29 hispida Pursh 

I 5 I 30 longistylis Nutt.

I 513 I psilostachya DC.

I 5132 pumila A. Gray

I 5 I 33 trifida L.

I 5I34 integrifolia(Muhl.)'T.\&G.

\section{Gaertneria}

(Franseria)

I5I35 acanthicarpa (Hook.) Britton (F. Hookeriana Nutt.)

I 5 I36 ambrosioides (Cav.) Kuntze

I 537 bipinnatifida (Nutt.) Kuntze I5138 dubia (Eastwood) Heller I 5139 Chamissonis (Less.) Kuntze I 5 I 40 viscida (Eastwood) Heller I 5I 4 I cordifolia (A. Gray) Kuntze I 5I 42 deltoidea (Torr.) Kuntze I 5 I43 discolor (Nutt.) Kuntze I5I44 dumosa (A. Gray) Kuntze I 5 I45 eriocentra (A. Gray) Kuntze I 5146 ilicifolia (A. Gray) Kuntze I 5 I 47 tenuifolia (A. Gray) Kuntze I 5 I48 tomentosa (A. Gray) Kuntze

\section{Xanthium}

15I49 acerosum Greene

I5I 50 acutum Greene

15I5I affine Greene

I 5 I 52 Californicum Greene

I 5I53 campestre Greene

I5I54 Canadense Mill.

I 5 I55 glanduliferum Greene

I 5 I 56 palustre Greene

I5I57 silphiifolium Greene

I ${ }^{1} 5^{8}$ speciosum Kearney

I 5 I 59 spinosum I.

I 5160 strumarium L.

I5I6I varians Greene

\section{Crassina}

(Zinnia)

I 5162 acerosa (DC.) Kuntze

I 5 I63 anomala (A. Gray) Kuntze I5I64 grandiflora (Nutt.) Kuntze I5I65 Peruviana (L.) Kuntze (Z. pauciflora L.)

I 5166 pumila (A. Gray) Kuntze

\section{Sanvitalia}

I5I67 Aberti A. Gray
I5I68 ocymoides DC.

\section{Heliopsis}

15169 gracilis Nutt.

I 5 I 70 helianthoides (L.) B. S. P.

(H. laevis Pers.)

I 5I7I parvifolia A. Gray

I5I72 scabra Dunal

\section{Eclipta}

15I73 alba (L.) Haussk.

\section{Sclerocarpus}

I 5 I 74 uniserialis (Hook.) B. \& H.

\section{Tetragonotheca}

I 5I75 helianthoides L.

I 5176 Ludoviciana A. Gray

I5I77 repanda A. Gray

I5I78 Texana Engelm. \& Gray

\section{Varilla}

I 5I79 Texana A. Gray

\section{Isocarpha}

I 5I80 oppositifolia R. Br.

\section{Rudbeckia}

I5I8I alismaefolia T. \& G.

I 5 I82 alpicola Piper

I5I83 amplexicaulis Vahl.

I5I 84 atrorubens Nutt.

I5I 85 bicolor Nutt.

I5186 Brittonii Small

I5I87 bupleuroides Shuttlw.

I 5 I 88 Californica A. Gray

I5189 fulgida Ait.

I 5I90 grandiflora C. C. Gmel

I5I9I heliopsidis 'T. \& G.

I 5192 heterophylla T. \& G.

I 5 I93 hirta L.

I 5I94 laciniata L.

I5I95 humilis A. Gray

I 5196 maxima Nutt.

I 5197 mollis A. Gray

I 5198 montana A. Gray

I 5199 nitida Nutt.

I 5200 longifolia A. Gray

I 5201 occidentalis Nutt.

I5202 spathulata Michx.

I 5203 speciosa Wender 

I 5204 subtomentosa Pursh

I5205 triloba L.

I 5206 pinnatiloba T. \& G.

I 5207 rupestris A. Gray

\section{Braumeria}

(Echinacea)

I5208 angustifolia (DC.) Heller

(B. Tennesseensis Beadle)

I 5209 pallida (Nutt.) Britton

I5210 purpurea (L.) Britton

\section{Ratibida}

\section{(Lepachys)}

I52II columnaris (Sims) D. Don

I52I 2 pulcherrima (DC.) D. Don I52I 3 peduncularis ( $T$. \& G.)

Barnhart

I 52 I 4 pinnata (Vent.) Barnhart

I521 5 Tagetes (James) Barnhart

\section{Gymnolomia}

I52I6 annua (Jones)

Rob. \& Greenm.

I52I7 longifolia Rob. \& Greenm.

I 52 I 8 multiflora (Nutt.) B. \& H.

I52I9 Porteri A. Gray

I 5220 tenuifolia (A. Gray) B. \& H.

\section{Zaluzania}

I522I Grayiana Rob. \& Greenm.

(Gymnolomia triloba A. Gray)

\section{Balsamorrhiza}

I5222 Balsamorrhiza (Hook.) Heller (B. Hookeri Nutt.)

I 5223 Bolanderi A. Gray

I5224 Careyana A. Gray

I5225 deltoides Nutt.

I5226 hirsuta Nutt.

I5227 incana Nutt.

I 5228 invenusta (Greene) Coville

(Helianthus invenustus Greene)

I5229 macrophylla Nutt.

I 5230 sagittata (Pursh) Nutt.

I523I terebinthacea (Hook.) Nutt.

\section{Borrichia}

I 5232 arborescens (L.) DC.

I 5233 frutescens (L.) DC.

\section{Serumeum}

I5234 trilobatum (I.) Kuntze (Wedelia carnosa Pers.)

Wyethia

I 5235 amplexicaulis Nutt.

I5236 angustifolia Nutt.

I 5237 Arizonica A. Gray

I $523^{8}$ coriacea A. Gray

I5239 glabra A. Gray

I 5240 helenioides Nutt.

I524I helianthoides Nutt.

I5242 lanceolata Howell

I 5243 longicaulis A. Gray

I 5244 mollis A. Gray

I 5245 ovata T. \& G.

I5246 reticulata Greene

I 5247 robusta Nutt.

I5248 scabra Hook.

\section{Tithonia}

I5249 Thurberi A. Gray

\section{Viguiera}

I.5250 canescens DC.

I 525 I cordifolia A. Gray

I 5252 helianthoides H. B. K.

I 5253 laciniata A. Gray

I5254 Parishii Greene

I5255 reticulata Wats.

I 5256 tephrodes A. Gray

\section{Helianthus}

I 5257 angustifolius L.

I $525^{8}$ annuus $\mathrm{L}$.

I 5259 argophyllus 'T. \& G.

I 5260 atrorubens L.

I 526I Bolanderi A. Gray

(H. scaberrimus Benth.)

I 5262 Californicus DC.

15263 Mariposanus A. Gray

I 5264 Utahensis A. Gray

$\mathrm{I} 5265$ ciliaris DC.

I 5266 cinereus T. \& G.

I5267 Cusickii A. Gray

I 5268 debilis Nutt.

I 5269 cucumeriformis A. Gray

I 5270 decapetalus L.

I 527 I multiflorus A. Gray

I5272 divaricatus L. 

I5273 doronicoides Lam.

I 5274 Douglasil T. \& G.

I 5275 exilis A. Gray

1 5276 Floridanus A. Gray

I5277 giganteus L.

I 5278 ambiguus T. \& G.

ז5279 glaucus Small

I 5280 gracilentus A. Gray

I 5281 grosse-serratus Martens

I 5282 hypoleucus A. Gray

I 5283 heterophyllus Nutt.

I 5284 hirsutus Raf.

I 5285 stenophyllus T. \& G.

I5286 trachyphyllus T. \& G.

I 5287 laetiflorus Pers.

I 5288 tricuspis T. \& G.

I 5289 laevigatus T. \& G.

I5290 longifolius Pursh

I5291 Maximiliani Schrad.

I 5292 microcephalus T. \& G. (H. parviflorus Bernh.)

I 5293 mollis Lam.

I5294 cordatus Wats.

I5295 Nuttallii T. \& G.

I 5296 occidentalis Riddell

I5297 Dowellianus 'T. \& G.

I 5298 plantagineus T. \& G.

I 5299 Oliveri A. Gray

I 5300 orgyalis DC.

I530r Parishii A. Gray

I5302 petiolaris Nutt.

I5303 canus Britton (var. canescens A. Gray)

I5304 patens (Lehm.) Rydb.

I5305 polyphyllus Small

I5306 pumilus Nutt.

I 5307 radula T. \& G.

I5308 scaberrimus Ell.

(H. rigidus Desf.)

I5309 Schweinitzii T. \& G.

I 53 IO strumosus L.

I53I I macrophyllus (Willd.)

I53 2 subrhomboideus Rydb.

I53I 3 tephrodes A. Gray

I53I 4 tomentosus Michx.

I53I5 tracheliifolius Mill.

r 53 I6 tuberosus L.
I53I7 subcanescens A. Gray

I 5318 verticillatus Small

\section{Melanther:}

I53I9 deltoidea Michx.

I5320 hastata Michx.

I532I lanceolata Benth.

\section{Flowrensia}

I5322 cernua DC.

\section{Spilanthes}

I 5323 repens (Michx.) Walt.

I5324 stolonifera DC.

\section{Encelia}

I 5325 Californica Nutt.

15326 calva (Engelm. \& Gray)

I 5327 eriocephala A.Gray [A.Gray

I 5328 paniculata A. Gray

I5329 exaristata A. Gray

I 5330 farinosa A. Gray

I 553 I frutescens A. Gray

I5332 grandiflora Jones

I5333 nutans Eastwood.

I 5334 scaposa A. Gray

I5335 subaristata A. Gray

I5336 viscida A. Gray

\section{Helianthella}

I 5337 argophylla (D. C. Eaton)

I 5338 Californica A.Gray [A.Gray

I5339 castanea Greene

I 5340 Douglasii T. \& G.

I 534 I grandiflora T. \& G.

I5342 microcephala A. Gray

I 5343 Nevadensis Greene

I5344 nudicaulis A. Gray

I5345 Parryi A. Gray

I 5346 quinquenervis(Hook.)A.Gray

I5347 Arizonica A. Gray

I 5348 tenuifolia T. \& G.

I 5349 uniflora (Nutt.) T. \& G.

\section{Verbesina}

(Includes Actinomeris)

I5350 alternifolia (L.) Britton

(A. squarrosa)

I 535 I aristata (Eil.) Heller

( $V$. nudicaulis A. Gray) 

I 5352 encelioides (Cav.) A. Gray

I 5353

I 5354 cana (DC.)Rob.\&Greenm. exanticulata

Rob. \& Greenm.

I5355 helianthoides Michx.

I 5356 heterophylla(Chapm.)A.Gray

I 5357 Lindheimeri Rob. \& Greenm.

I $535^{8}$ longifolia A. Gray

i 5359 microptera DC.

I 5360 mollissimaRob.\&Greenm.

I 536 I nana (A.Gray)Rob. \&Greenm.

I 5362 occidentalis (L.) Walt.

I5363 paniculata (Walt.) Heller

(A. alba T. \& G.)

I 5364 Rothrockii Rob. \& Greenm.

I 5365 Texana Buckley

I 5566 Virginica L.

I5367 laciniata (Nutt.) A. Gray

I5368 Warei A. Gray

I 5369 Wrightii A. Gray

\section{Zexmenia}

I5370 brevifolia A. Gray

I 537 I hispida (H. B. K.) A. Gray

I 5372 podocephala A. Gray

\section{Synedrella}

I 5373 nodiflora (L.) Gaertn.

\section{Calyptrocarpus}

15374 vialis Less.

(Synedrella vialis A. Gray)

\section{Coreopsis}

I5375 Atkinsoniana Dougl.

I 5376 auriculata L.

I 5377 cardaminefolia (DC.) T. \& G.

I 5378 crassifolia Ait.

I 5379 delphinifolia Lam.

I 5380 Drummondii (Ion) T. \& G.

1538 I Wrightii A. Gray

I 5382 gladiata Walt.

I 5383 grandiflora Hogg

I 5384 Harveyana A. Gray

I 5385 helianthoides Beadle

I5386 integrifolia Poir.

I 5387 lanceolata L.

I 5388 angustifolia T. \& G.

I 5389 villosa Michx.

I5390 latifolia Michx.
I 539 I Leavenworthii T. \& G.

I5392 Garberi A. Gray

I5393 Lewtonii Small

I 5394 longifolia Small

I 5395 major Walt.

(C. senifolia Michx.)

I 5396 linearis Small

I 5397 nudata Nutt.

I 5398 Nuecensis Heller

(C. coronata Hook.)

I5399 Oemleri Ell.

(C. senifolia var. stellata T. \& G.)

I 5400 palmata Nutt.

I540I pubescens E11.

I 5402 rosea Nutt.

${ }_{5403}$ tinctoria Nutt.

I5404 tripteris L.

I 5405 verticillata $L$.

\section{Leptosyne}

I 5406 Arizonica A. Gray

I 5407 Bigelovii A. Gray

I 5408 calliopsidea (DC.) A. Gray

I 5409 nana A. Gray

I 54 IO Douglasii DC.

I54I I gigantea Kellogg

I 54 I 2 maritima (Nutt.) A. Gray

r 541 3 Stillmani A. Gray

\section{Heterospermum}

I54I 4 pinnatum Cav.

\section{Thelesperma}

I54I5 ambiguum A. Gray

I 5416 gracile ('Torr.) A. Gray

I 53 I 7 longipes A. Gray

I54I 8 marginatum Rydb.

I 5419 subnudum A. Gray

I 5420 simplicifolium. A. Gray

(T. subsimplicifolium A. Gray)

I542 I trifidum (Poir.) Britton

(T. filifolium A. Gray)

\section{Bidens}

I 5422 aristosa (Michx.) Britton (Corcopsis aristosa Michx.)

I 5423 Beckii Torr.

I 5424 bidentoides (Nutt.) Britton (C. bidentoides T. \& G.)

I 5425 Bigelovii A. Gray 

I 5426 bipinnata I.:

I 5427 cernula L.

I5428 elliptica Wiegand

I 5429 integra Wiegand

I 5430 comosa (A. Gray) Wiegand

I543I acuta Wiegand

I5432 connata Muhl.

I 5433 pinnata Wats.

I5434 coronata (Li.) Fisch. (C. aurea Ait.)

I 5435 dentata (Nutt.) Wiegand

I 5436 discoidea (T. \& G.) Britton (C. discoidea T. \& G.)

I 5437 frondosa L.

I $543^{8}$ puberula Wiegand

I5439 heterophylla Ortega

I5440 Wrightii A. Gray

I 544I heterosperma A. Gray

I 5442 involucrata (Nutt.) Britton (C. involucrata Nutt.)

I 5443 laevis (L.) B. S. P. (B. chrysanthemoides Michx.)

I 5444 Lemmoni A. Gray

15445 lencantha Willd.

I3446 melanocarpa Wiegand

I5447 pallida Wiegand

I5448 Nashii Small

I5449 pilosa L.

I 5450 procera Don

I545I tenuisecta A. Gray

I 5452 trichosperma (Michx.)Britton

(Coreopsis trichosperma Michx)

I5453 tenuiloba(A.Gray)Britton

I5454 vulgatus Greene

\section{Wootonia}

I5455 parviflora Greene

\section{Cosmos}

I 5456 bipinnatus exaristatus DC.

I 5457 caudatus H. B. K.

I $545^{8}$ parviflorus $\mathrm{H}$. B. K.

\section{Actinospermum}

(Baldwinia)

I5459 angustifolium (Pursh) Torr.

I 5460 uniflorum (Nutt.) Barnhart

\section{Bebbia}

I546r juncea (Benth.) Greene

\section{Galinsoga}

I 5462 Carascana (DC.) Sch. Bip.

I5463 parviflora Cav.

I5464 hispida Cav.

\section{Marshallia}

I5465 angustifolia Pursh

I 5466 Cyananthera E.11.

I 5467 caespitosa Nutt.

I5468 lacinarioides Small

r5469 lanceolata Pursh

I5470 platyphylla Curtis

I 547 I trinervia (Walt.) Porter (M. latifolia Pursh)

\section{Ptilonella}

(Blepharipappus)

I 5472 laevis (A. Gray) Greene

I 5473 scabra Nutt.

I5474 subcalva (A.Gray) Greene

\section{Madia}

I5475 anomala Greene

I 5476 capitata Nutt.

I 5477 citriodora Greene

I 5578 congesta Nutt.

I 5479 dissitiflora (Nutt.) T. \& G.

I 5480 glomerata Hook.

I548I polycarpha Greene

I 5482 racemosa (Nutt.) T. \& G.

I 5483 sativa Molina

I5484 tenella Greene

\section{Anisocarpus}

(Madia in part)

15485 Bolanderi A. Gray

I 5486 madioides Nutt.

(M. Nuttallii A. Gray)

I 5487 radiatus (Kellogg) Greene

I5488 Rammii Greene

I5489 Yosemitanus (Parry) Greene

\section{Madaria}

(Madia in part)

I 5490 corymbosa DC.

I 549 I densifolia Greene

I 5492 elegans (Don) DC.

I 5493 hispida DC. 



\section{Hemizonia}

I 5494 anomala A. Gray

I 5495 citrina Greene

I 5496 Clemantina T.S. Brandegee

I 5497 Clevelandi Greene

I 5498 Congdoni Rob. \& Greenm.

I 5499 congesta DC.

I 5500 floribunda A. Gray

I55O I luzulaefolia DC.

I 5502 lutescens Greene

I 5503 paniculata A. Gray

I 5504 Streetsii A. Gray

I5505 Wheeleri A. Gray

\section{Centromadia}

(Hemizonia in part)

I 5506 Fitchii (A. Gray) Greene

I 5507 maritima Greene

I 5508 Parryi Greene

I 5509 perennis Greene

I55IO pungens (H. \& A.) Greene

I 55 I I rudis Greene

\section{Holocarpha}

r55 I 2 macradenia (DC.) Greene (Hemizonia macradenia DC.)

\section{Deinandra}

(Hemizonia in part)

I $55^{\text {I } 3}$ fasciculata DC.

I5514 Heermanni Greene

I $55^{\text {I } 5}$ Kelloggii Greene

I $55^{1} 6$ Lobbii Greene

I 55I 7 Wrightii (A. Gray) Greene

\section{Zonanthemis}

(Hemizonia in part)

$\mathrm{r}_{5518} 8$ angustifolia (DC.) Greene

I $55^{19}$ corymbosa (DC.) Greene

\section{Calycadenia}

(Hemizonia in part)

I 5520 bicolor Greene

I $55^{21}$ campestris Greene

I 5522 cephalotes DC.

I 5523 ciliosa Greene

15524 elegans Greene

15525 Fremonti A. Gray

I 5526 hispida Greene

I 5527 mollis A. Gray
I 5528 multiglandulosa DC.

I 5529 oppositifolia Greene

I 5530 pauciflora A. Gray

I 553I ramulosa Greene

I 5532 scabrella (Drew) Greene

I 5533 spicata Greene

I 5534 tenella (Nutt.) T. \& G.

I 5535 truncata DC.

I 5536 villosa DC.

\section{Blepharizonia}

I5537 laxa Greene

I $553^{8}$ plumosa (Kellogg) Greene

\section{Harpaecarpus}

I5539 exigua (Sm.) A. Gray (Madia filipes A. Gray)

I 5540 minimus (A. Gray) Greene

(Hemizonella minima A. Gray)

I 554I parvulus (A. Gray) Greene

(Hemizonella Durandi A. Gray)

\section{Lagophylla}

I5542 congesta Greene

I 5543 dichotoma Benth.

I 5544 glandulosa A. Gray

I 5545 ramosissima Nutt.

I5546 serrata Greene

\section{Holozonia}

I 5547 filipes (H. \& A.) Greene

\section{Blepharipappus}

(Layia)

I 5548 carnosus (Nutt.) Greene

I 5549 chrysanthemoides (DC.)

I5550 Douglasii (Hook.) Greene (L. Calliglossa A. Gray)

I555I elegans (Nutt.) Greene

I 5552 Fremonti (T. \& G.) Greene

r 5553 gaillardioides (H.\&A.)Greene

I 5554 glandulosus Hook.

I 5555 graveolens Greene

I 5556 heterotrichus (DC.) Greene

I 5557 hieracioides (DC.) Greene

I $555^{8}$ hispidus Greene

I 5559 Jonesii (A. Gray) Greene

I 5560 nemorosus Greene

I556I nutans Greene 

I5562 Oreganus Greene (L. Douglasii H. \& A.)

I $55^{6} 3$ pentachaetus (A.Gray)Greene I 5564 platyglossus (F. \& M.) Greene

Achyrachaena

I 5565 mollis Schaner

\section{Jaumea}

I 5566 carnosa (Less.) A. Gray

Venegasia

${ }^{1} 5567$ carpesioides DC.

\section{Clappia}

I 5568 suaedefolia A. Gray

\section{Psilostrophe}

\section{(Riddellia)}

I 5569 Cooperi (A. Gray) Greene

I 5570 gnaphalodes DC.

I 557 I tagetinae (Nutt.) Greene

I 5572 sparsiflora(A.Gray)Greene

\section{Baileya}

I5573 multiradiata Harv. \& Gray

I 5574 nervosa Jones

I 5575 pauciradiata Harv. \& Gray

I 5576 pleniradiata Harv. \& Gray

\section{Whitneya}

I 5577 dealbata A. Gray

\section{Perityle}

I5578 aglossa A. Gray

I 5579 coronopifolia A. Gray

I 5580 dissecta A. Gray

I558I effusa (A. Gray) Rose

I5582 Emoryi Torr.

I5583 leptoglossa A. Gray

I $55^{84}$ microglossa Benth.

I $55^{8} 5$ nuda Torr.

15586 Parryi A. Gray

I $55^{87}$ plumigera A. Gray

I 5588 Rothrockii Rose

I 5589 Vaseyi Coult.

\section{Laphamia}

I5590 angustifolia A. Gray

I 559I bisetosa Jones

I 5592 cernua Greene

I5593 ciliata L. H. Dewey
I 5594 cinerea A. Gray

I 5595 congesta Jones

I 5596 fastigiata T. S. Brandegee

I 5597 Gilensis Jones

I 5598 gracilis Jones

I 5599 halimifolia A. Gray

I 5600 intricata 'T. S. Brandegee

I 5601 Lemmoni A. Gray

I 5602 pedata A. Gray

I 5603 Lindheimeri A. Gray

I 5604 megalocephala Wats.

I 5605 Palmeri A. Gray

I 5606 tenella Jones

I 5607 rupestris A. Gray

I 5608 Stansburii A. Gray

I5609 Toumeyi Rob. \& Greenm.

\section{Eatonella}

I56ro nivea (D. C. Eaton) A. Gray

\section{Lembertia}

I 56I I Congdoni (A. Gray) Greene

(Eatonella Congdoni A. Gray)

\section{Pericome}

I 56 I 2 caudata A. Gray

\section{Crockeria}

I56I3 chrysantha Greene

\section{Burrielia}

I56I 4 microglossa DC.

\section{Baeria}

15615 affinis (Nutt.) A. Gray

I 56I6 anthemoides (Nutt.) A. Gray

I 56I 7 aristosa (A. Gray) Howell

I 56I 8 Burkei Greene

I 56I9 carnosa Greene

I 5620 chrysostoma F. \& M.

I562I Clevelandi A. Gray

I 5622 consanguinea Greene

I 5623 coronaria (Nutt.) A. Gray

I 5624 curta A. Gray

I 5625 debilis Greene

I 5626 Fremonti (Benth.) A. Gray

I 5627 gracilis (DC.) A. Gray

I 5628 hirsutula Greene

I 5629 leptalea A. Gray

I 5630 macrantha A. Gray

I 563 I maritima A. Gray 

I 5634 mutica (Nutt.) A. Gray

5635 Palmeri Clementina A. Gray

I 5636 Parishii Wats.

I 5637 platycarpha A. Gray

I 5638 tenella (Nutt.) A. Gray

I 5639 uliginosa (Nutt.) A. Gray

\section{Lasthenia}

(Baeria in part)

I5640 Burkei Greene

I 564 I Californica DC.

I 5642 conjugens Greene

I 5643 Fremonti (A. Gray) Greene I 5644 glaberrima DC.

I 5645 glabrata Lindl.

I5646 Coulteri A. Gray

\section{Monolopia}

I5647 gracilens A. Gray

I 5648 major DC.

I5649 lanceolata A. Gray

I 5650 minor DC.

\section{Flaveria}

1565I angustifolia (Cav.) Pers.

I 5652 chloraefolia A. Gray

I 5653 linearis Lag.

I5654 repanda Lag.

\section{Syntrichopappus}

I 5655 Fremonti A. Gray

I 5656 Lemmoni A. Gray

\section{Sartwellia}

I 5657 flaveriae A. Gray

\section{Biolettia}

I5658 riparia Greene

\section{Schkuria}

I5659 Kopkirkia A. Gray

I 5660 Wrightii A. Gray

\section{Hymenopappus}

r566I arenosus Heller

I 5662 artemisiaefolius DC.

I 5663 Carolinensis (Lam.) Porter (H. scabiosaeus L'Her.)

I 5664 corymbosus T. \& G.

I 5665 filifolius Hook.

I 5666 fiavescens A. Gray

I 5667 gloriosus Heller
I 5668 lugens Greene

I5669 luteus Nutt.

I 5670 Mexicanus A. Gray

I567 I obtusifolius Heller

I5672 radiatus Rose

I5673 tennifolius Pursh

\section{Hymenothryx}

I5674 Wislizeni A. Gray

I 5675 Wrightii A. Gray

\section{Florestina}

I 5676 tripteris DC.

\section{Palafoxia}

I5677 Feayi A. Gray

I 5678 linearis Lag.

\section{Polypteris}

I 5679 callosa (Nutt.) A. Gray

I 5680 Hookeriana ('T.\& G.) A. Gray

I 568I integrifolia Nutt.

I 5682 Texana (DC.) A. Gray

\section{Eriophyllum}

ז 5683 achillaeoides (DC.) Greene I5684 ambiguum A. Gray

I 5685 arachnoideum (F.\&L.) Greene I 5686 aureum T. S. Brandegee ${ }_{15687}$ bahiaefolium (Benth.) Greene (Monolopia bahiaefolia Benth.) I5688 caespitosum Dougl.

I 5689 confertiflorum (DC.) A. Gray

I 5690 discoideum Greene

I 569I laxiflorum A. Gray

I5692 trifldum A. Gray

I5693 Congdoni T. S. Brandegee

I 5694 croceum Greene

I 5695 gracile A. Gray

I 5696 grandiflorum (A.Gray)Greene I 5697 Heermanni (Durand) Greene

(Monolopia Heermanni Durand)

I 5698 integrifolium (Hook.) Greene

I5699 Jepsoni Greene

I 5700 lanceolatum Howell

I570 lanatum (Pursh) Forbes

I 5702 leucophyllum (DC.) Rydb.

I 5703 multiflorum (Nutt.) Rydb.

15704 Nevinii A. Gray

I 5705 obovatum Greene

r 5706 paleaceum T. S. Brandegee 

I 5707 pedunculatum Heller

15708 speciosum Greene

I 5709 stachadifolium Lag.

157 Io depressum Greene

I 57II tanacetifolium Greene

I5712 ternatum Greene

I5713 Watsoni A. Gray

\section{Actinolepis}

(Eviophyllum in part)

I57I4 lanosa A. Gray

I57I5 multicaulis DC.

I5716 nubigena Greene

I57 7 Pringlei (A. Gray) Greene

15718 Wallacei A. Gray

\section{Amblyopappus}

I57I9 pusillus H. \& A.

\section{Rigiopappus}

I5720 leptocladus A. Gray

I 5721 longiaristatus A. Gray

\section{Chaenactis}

I 5722 achillaefolia H. \& A.

I 5723 alpina (A. Gray) Jones

I 5724 artemisiaefolia A. Gray

I 5725 attenuata A. Gray

I 5726 brachypappa A. Gray

I5727 carphoclinia A. Gray

I 5728 Cusickii A. Gray

I 5729 Douglasii (Hook.) H. \& A.

I5730 montana Jones

I 573I floribunda Greene

I5732 Fremonti A. Gray

I 5733 glabriuscula DC.

I5734 gracilenta Greene

I5735 heterocarpha A. Gray

I 5736 lacera Greene

I5737 lanosa DC.

I $573^{8}$ macrantha D. C. Eaton

I 5739 Nevadensis (Kellogg) A.Gray

I5740 Nevii A. Gray

r574I Orcuttiana (Greene)

15742 Parishii A. Gray

S. B. Parish

I5743 pedicularia Greene

I5744 santolinoides Greene

I5745 scaposa Eastwood

I 5746 stevioides H. \& A.
I 5747 suffrutescens A. Gray

I5748 tanacetifolia A. Gray

I5749 tenuifolia Nutt.

I5750 Xantiana A. Gray

\section{Orochaenactis}

(Chaenactis in part)

I $575^{\text {I }}$ thysanocarpha (A. Gray)

\section{Bahia}

Coville

I5752 absinthifolia Benth.

I 5753 dealbata A. Gray

I 5754 Bigelovii A. Gray

I 5755 biternata A. Gray

I 5756 desertorum Jones

I 5757 dissecta (A. Gray) Britton

(B. chrysanthemoides A. Gray)

I $575^{8}$ Neo-Mexicana A. Gray

I 5759 nudicaulis A. Gray

I 5760 oblongifolia A. Gray

I 576I oppositifolia Nutt.

I5762 Palmeri Wats.

I 5763 pedata A. Gray

I 5764 Woodhousei A. Gray

\section{Hulsea}

I 5765 algida A. Gray

I 5766 brevifolia A. Gray

I 5767 Californica 'T. \& G.

I 5768 carnosa Rydb.

I5769 heterochroma A. Gray

I 5770 nana A. Gray

I 5771 Larseni A. Gray

I 5772 vestita A. Gray

\section{Trichoptilium}

I 5773 incisum A. Gray

\section{Tetraneuris}

(Actinella in part)

I 5774 acaulis (Pursh) Greene

r 5775 caespitosa Aven Nelson

I 5776 argentea (A. Gray) Greene

I5777 Arizonica Greene

I 5778 brevifolia Greene

I 5779 depressa ('T. \& G.) Greene

I 5780 fastigiata Greene

I 578I glabra (Nutt.) Greene

I5782 herbacea Greene 

${ }_{1} 5783$ incana Aven Nelson

15784 Ivesiana Greene

I 5785 lanata (Nutt.) Greene

I 5786 leptoclada (A. Gray) Greene

15787 linearifolia (Hook.) Greene

I 5788 linearis (Nutt.) Greene

I 5789 Mancosensis Aven Nelson

15790 scaposa (DC.) Greene

1579 I simplex Aven Nelson

15792 Torreyana (Nutt.) Greene

I5793 trinervata Greene

\section{Rydbergia}

(Actinella in part)

15794 glabrata (Porter) Greene

(Actinella Brandegei Porter)

I 5795 grandiflora ('T. \& G.) Greene

\section{Macdougalia}

(Actinella in part)

r5796 Bigelovii (A. Gray) Heller

\section{Picradenia}

(Actinella in part)

I 5797 biennis (A. Gray) Greene

I5798 canescens (D. C. Eaton)

Greene

\section{5799 Cooperi (A. Gray) Greene}

I 5800 floribunda (A. Gray) Greene

I580I Lemmoni Greene

I5802 ligulaeflora Aven Nelson

(P. pumila Greene)

I 5803 macrantha Aven Nelson

I 5804 multiflora (Buckley) Greene

I 5805 Richardsonii Hook.

I 5806 Rusbyi (A. Gray) Greene

I5807 Texana (Rose) Greene

I 5808 Vaseyi (A. Gray) Greene

\section{Helenium}

I5809 amphibolum A. Gray

I 58 Io autumnale L.

I 58I I grandiflorum Howell

I 5812 pubescens (Ait.) Britton

I58I3 brevifolium A. Gray

I58I4 Curtisii A. Gray

I58I5 elegans DC.

I 58 I 6 fimbriatum A. Gray

I58I7 laciniatum A. Gray

I5818 microcephalum DC.
I 58 I9 montanum Nutt.

I 5820 nudiflorum Nutt.

I 582 I Nuttallii A. Gray

I 5822 incisum A. Gray

I 5823 ooclinum A. Gray

I 5824 parviflorum Nutt.

I 5825 quadridentatum Labill

I5826 tenuifolium Nutt.

I 5827 badium A. Gray

I 5828 Thurberi A. Gray

I 5829 vernale Walt.

\section{Heleniastrum}

(Helenium in part)

I 5830 Bigelovii (A. Gray) Kuntze

I 583I Bolanderi (A. Gray) Kuntze

I $5^{8} 3^{2}$ puberulum (DC.) Kuntze

I5833 rivulare Greene

\section{Dugaldea}

(Helenium in part)

I5834 Hoopesii (A. Gray) Rydb.

\section{Amblyolepis}

r 5835 setigera DC.

\section{Gaillardia}

I 5836 acaulis A. Gray

I 5837 amblyodon Gay

I $583^{8}$ aristata Pursh

I 5839 Arizonica A. Gray

I5840 lanceolata Michx.

I 584I Mexicana A. Gray

I 5842 multiceps Greene

I 5843 pinnatifida Torr.

I 5844 pulchella Foug.

I 5845 picta (Don) A. Gray

I 5846 suavis (Engelm. \& Gray)

(G. simplex Scheele) [B. \& R.

I 5847 spathulata A. Gray

\section{Plummera}

I 5848 floribunda A. Gray

Blennosperma

I5849 Californicum (DC.) 'T. \& G.

\section{Nicolletia}

I $585^{\circ}$ Edwardsii A. Gray

I 585 I occidentalis A. Gray 

Tagetes

I 5852 Lemmoni A. Gray

I5853 micrantha Cav.

\section{Dysodia}

I 5854 Cooperi A. Gray

I 5855 papposa (Vent.) A. S. Hitchc. (D. chrysanthemoides Lag.)

I 5856 porophylloides A. Gray

\section{Hymenatherum}

I $5^{8} 57$ acerosum (DC.) A. Gray

I $585^{8}$ concinnum A. Gray

I 5859 Hartwegi A. Gray

I 5860 micropoides (DC.) Heller. (H. Gnaphaliopsis A. Gray)

I586I Neo-Mexicanum A. Gray

I 5862 pentachaetum DC.

I 5863 polychaetum A. Gray

I 5864 tagetoides ('T. \& G.) A. Gray

I 5865 tenuilobum DC.

I 5866 Thurberi A. Gray

I5867 Treculii A. Gray

I5868 Wrightii A. Gray

\section{Thymophylla}

(Hymenatherum in part)

I 5869 aurea (A. Gray) Greene

I 5870 Greggi A. Gray

\section{Porophyllum}

I587 I gracile Benth.

I 5872 macrocephalum DC.

I5873 scoparium A. Gray

\section{Chrysactinia}

I5874 Mexicana A. Gray

\section{Pectis}

${ }_{5} 875$ angustifolia Torr.

15876 subaristata A. Gray

I 5877 ciliaris L.

I 5878 Coulteri Harv. \& Gray

I 5879 filipes A. Gray

I 5880 imberbis A. Gray

I588I Lessingii Fernald

I 5882 linifolia L.

I 5883 marginalis Fernald

I5884 longipes A. Gray

I 5885 papposa Harv. \& Gray
I 5886 prostrata Cav.

I 5887 cylindrica Fernald

I 5888 punctata Jacq.

I 5889 Rusbyi Greene

I589o tenella DC.

\section{Anthemis}

I $589 \mathrm{I}$ arvensis $\mathrm{L}$.

I 5892 Cotula L。

I 5893 mixta L.

I 5894 nobilis L.

I 5895 tinctoria L.

\section{Achillea}

I 5896 borealis Bong.

I 5897 Californica Pollard

I5898 gigantea Pollard

I 5899 lanulosa Nutt.

I 5900 alpicola Rydb.

I590I ligustica All.

I 5902 Millefolium L.

I 5903 multiflora Hook.

I 5904 Ptarmica L.

\section{Leucampyx}

I5905 Newberryi A. Gray

\section{Matricaria}

I5906 Chamomilla L.

I 5907 grandiflora (Hook.) Britton

I 5908 inodora L.

I 5909 matricarioides (Less.) Porter (M. discoidea DC.)

I5910 occidentalis Greene

\section{Chrysanthemum}

I59II arcticum L.

I 5912 Balsamita L.

I 59I3 tanacetoides Boiss.

I 5914 bipinnatum L.

I59I5 Indicum L.

I 5916 integrifolium Richards.

I 5917 Leucanthemum L.

I 59 I 8 Parthenium (L.) Pers.

r5919 segetum Lobel

\section{Tanacetum}

I 5920 camphoratum Less.

I 592 I Huronense Nutt.

I5922 vulgare $\mathrm{L}$. 



\section{Sphaeromeria}

(Tanacetum in part)

I 5923 argentea $\mathrm{Nutt.}$

(T. Nuttallii T. \& G.)

I 5924 cana (D. C. Eaton) Heller

I 5925 capitata Nutt.

15926 diversifolia (D. C. Eaton)

Heller

I 5927 potentilloides (A.Gray)Heller I 5928 simplex (AvenNelson) Heller

\section{Cotula}

I 5929 australis Hook.

I5930 coronopifolia L.

\section{Soliva}

I593I nasturtiifolia DC.

I 5932 sessilis R. \& P.

\section{Artemisia}

I 5933 Abrotanum L.

I 5934 absinthium L.

I 5935 annua $\mathrm{L}$.

I5936 arbuscula Nutt.

I 5937 aromatica Aven Nelson

I 5938 biennis Willd.

I 5939 Bigelovii A. Gray

I 5940 Bolanderi A. Gray

I 594 I borealis Pall.

I 5942 Wormskioldii Bess.

I 5943 Californica Less.

I 5944 cana Pursh

I 5945 viscidula Osterhont

I 5946 Canadensis Michx.

I 5947 candicans Rydb.

I 5948 caudata Michx.

I 5949 Coloradensis Osterhont

I $595^{\circ}$ discolor Dougl.

I 595I dracunculoides Pursh

I 5952 elatior ('T. \& G.) Rydb.

I 5953 filifolia Torr.

I 5954 floccosa Rydb.

I 5955 Forwoodii Wats.

I 5956 franserioides Greene

I 5957 frigida Willd.

I $595^{8}$ glauca Pall.

I 5959 globularia Cham.

I 5960 glomerata Ledeb.

I 596r gnaphalodes Nutt.
I 5962 gracilenta Aven Nelson

I 5963 graveolens Rydb.

r 5964 Groenlandica Wormsk.

I 5965 heterophylla Nutt.

I 5966 incompta Nutt.

I5967 Kansana Britton

I 5968 latiloba (Nutt.) Rydb.

I 5969 Lindleyana Bess.

I 5970 longifolia Nutt.

1597 I Ludoviciana Nutt.

I5972 Mexicana Willd.

I5973 Pacifica A. Gray

I5974 microcephala Wooton

I 5975 Natronensis Aven Nelson

I5976 Norvegica Fries

I 5977 nova Aven Nelson

I 5978 Palmeri A. Gray

I5979 Parishii A. Gray

I5980 Parryi A. Gray

I598I pancicephala Aven Nelson

I 5982 Pattersoni A. Gray

I 5983 pedatifida Nutt.

I 5984 Pontica L.

I 5985 Prescottiana Bess.

I5986 procera Willd.

I 5987 Purshiana Bess.

I 5988 pycnocephala DC.

I5989 pygmaea A. Gray

I 5990 rhizomata Aven Nelson

I 599I pabularis Aven Nelson

I5992 Richardsoniana Bess.

I5993 rigida A. Gray

I 5994 Rothrockii A. Gray

I5995 scopulorum A. Gray

I 5996 pedatifida Nutt.

I 5997 serrata Nutt.

I 5998 spiciformis Osterhout

I 5999 spinescens D. C. Eaton

I600o Stelleriana Bess.

I600 I subglabra Aven Nelson

16002 tenuis Rydb.

I6003 integerrima Rydb.

I6004 Tilesii Ledeb.

I6005 tridentata Nutt.

I6006 angustifolia A. Gray

I6007 tripartita Rydb.

(A. trifida Nutt.)

I6008 vulgaris L. 

I6009 Wrightii A. Gray

\section{Tussilago}

I6oro Farfara L.

\section{Petasites}

I6or I frigida (L.) Fries

I6or 2 nivalis Greene

I6or 3 palmata (Ait.) A. Gray

I60I 4 Petasites (L.) Karst.

(P. officinalis Moench)

I6or5 sagittata (Pursh) A. Gray

\section{Erechtites}

I6or6 hieracifolia (L.) Raf.

\section{Raillardella}

I60I7 argentea A. Gray

I6018 Muirii A. Gray

I60I9 Pringlei Greene

I6020 scaposa A. Gray

I602I Eiseni A. Gray

\section{Lepidospartium}

I6022 latisquamum Wats.

r6o23 squamatum A. Gray

I6024 striatum Coville

\section{Haploesthes}

I6025 Greggii A. Gray

\section{Arnica}

I6026 acaulis (Walt.) B. S. P. (A. mudicaulis Nutt.)

I6027 alpina (L.) Olin

I 6028 Lessingii T. \& G.

I6029 amplexifolia Rydb.

(A. amplexicaulis Nutt.)

I6030 caespitosa Aven Nelson

r603 I cernuua Howell

I6032 Chamissonis Less.

I6033 longinodosa Aven Nelson

I6034 Columbiana Aven Nelson

I 6035 cordifolia Hook.

r6o36 denudata Greene

I6037 canescens Greene

I6038 discoidea Benth.

I6039 eradiata (A. Gray) Heller

(A. Parryi A. Gray)

16040 exigua Aven Nelson

I604I foliosa Nutt.

I6042 incana A. Gray
I6043 fulgens Pursh

I6044 gracilis Rydb.

I6045 Grayi Heller

(A. cordifolia var. eradiata A. Gray)

I6046 latifolia Bong.

I6047 viscidula A. Gray

I6048 longifolia D. C. Eaton

r6049 Merriami Greene

I6050 mollis Hook.

I605I monocephala Rydb.

16052 Nevadensis A. Gray

I6053 obtusifolia Less.

I6054 ocreata Aven Nelson

I6055 parviflora A. Gray

I6056 pedunculata Rydb.

I6057 polycephala Aven Nelson

I6058 pumila Rydb.

I6059 Rydbergii Greene

I606o Sonnei Greene

r606I spathulata Greene

I6062 subplumosa Greene

I 6063 Unalaschensis Less.

I6064 viscosa A. Gray

\section{Crocidium}

I6065 multicaule Hook.

\section{Bartlettia}

I6066 scaposa A. Gray

\section{Cacaliopsis}

I6067 Nardosmia A. Gray

\section{Luina}

I6068 hypoleuca Benth.

I6069 Californica A. Gray

\section{Raineria}

I6070 stricta Greene

(Luina Piperi Robinson)

\section{Peucephyllum}

1607I Schottii A. Gray

\section{Psathyrotes}

I6072 annua (Nutt.) A. Gray

16073 pilifera A. Gray

I6074 ramosissima (Torr.) A. Gray

I6075 scaposa A. Gray 



\section{Mesadenia}

(Cacalia)

I6076 atriplicifolia (L.) Raf.

16077 diversifolia ('T. \& G.) Greene I6078 Floridana (A. Gray) Greene I6079 lanceolata (Nutt.) Raf. I6080 ovata (Walt.) Raf. I608I reniformis (Muhl.) Raf. I6082 tuberosa (Nutt.) Britton

\section{Synosma}

(Cacalia in part)

I6083 suaveolens (L.) Raf.

\section{Tetradymia}

16084 canescens DC.

16085 comosa R. Gray I6086 glabrata A. Gray I6087 inermis Nutt. I6088 multicaulis Aven Nelson I6089 Nuttallii T. \& G. I6090 spinosa H. \& A. I609I stenolepis Greene

\section{Senecio}

I6092 accedens Greene I6093 Actinella Greene I6094 acutidens Rydb.

I6095 admirabilis Greene I6096 alpicola Rydb. r6097 altus Rydb. I6098 amplectens A. Gray I6099 taraxacoides A. Gray I6roo ampullaceus Hook. I6IOI andinus Nutt. (A. serra var. integriusculus A.Gray) I6102 antennariifolius Britton I6Io3 aphanactis Greene I6I04 arachnoideus Rydb. I6I05 Arizonicus Greene I6I06 aronicoides T. \& G. 16107 astephanus Greene I6Io8 atratus Greene I6Io9 atriapiculatus Rydb. (S. Columbianus Greene)

r6rio aurellus Rydb.

I6III aureus L.

I6I 2 Balsamitae Muhl. I6I I3 Bernardinus Greene
I6I 4 Bigelovii A. Gray

I6I I 5 blitoides Greene I6I16 Blochmanae Greene I6II7 Bolanderi A. Gray I6 I 8 Breweri Davy 16 I 9 Californicus DC. I6 I 20 candidissimus Greene I6I 2 I canovirens Rydib. I6I22 canus Hook. r6i23 Cardamine Greene I6I 24 carthamoides Greene r6I25 caulanthifolius Davy I6 26 cernuus A. Gray I6I 27 chloranthus Greene I6I 28 Cineraria DC.

I6r29 Clarkianus A. Gray I6I30 Clevelandi Greene I6r3I cognatus Greene I6r 32 compactus (A. Gray) Rydb. I6I33 condensatus Greene I6I 34 cordatus Nutt. I6I35 Covillei Greene I6I 36 crassulus A. Gray I6I 37 crocatus Rydb. r6r38 cymbalarioides Nutt.

(S. aureus var. borealis T. \& G.) I6I39 dimorphophyllus Greene r6r40 discoideus (Hook.) Britton I6I4I dispar Aven Nelson I6I 42 Douglasii DC. I6r43 Earlei Small I6I44 Elmeri Piper I6I45 eremophilus Richards. I6I46 eurycephalus T. \& G. I6r47 exaltatus Nutt. I6I 48 fastigiatus Nutt. I6I49 fedifolius Rydb. I6I5o Fendleri A. Gray I6I 51 flavovirens Rydb. 16I52 flavulus Greene r6I53 Flettii Wiegand I6r 54 foetidus Howell I6r 55 Franciscanus Greene I6I56 Fremontii T. \& G. I6I 57 frigidus Less. I6I58 fulgens Rydb. I6I59 Gibbonsii Greene I6I60 glancescens Rydb. 

I6I6I glaucifolius Rydb.

I6162 gracilis Pursh

16163 Greenei A. Gray

I6I64 Greggii Rydb.

I6I65 Halli Britton

16166 Hartianus Heller

16167 Hartwegi Benth.

I6I68 hesperius Greene

16169 Holmii Greene

I6r 7o Hookeri T. \& G.

I6I7I Howellii Greene

16172 Huachucanus A. Gray

I6I73 hydrophiloides Rydb.

16I74 hydrophilus Nutt.

I6I75 Pacificus Greene

16I76 Idahoensis Rydb.

16I 77 ilicetorum Davidson

16178 imbricatus Greene

16I79 indecorus Greene

I6I80 integerrimus Nutt.

I6I8I invenustus Greene

I6182 ionophyllus Greene

I6I83 Jacobaea L.

I6I84 Jonesii Rydb.

I6I85 lactucinus Greene

I6I86 laetiflorus Greene

I6r87 latus Rydb.

I6188 Laynae Greene

16189 Lemberti Greene

I6rgo Lemmoni A. Gray

16I9I leptolepis Greene

I6r92 longilobus Benth.

I6I93 longipetiolatus Rydb.

I6r94. lugens Richards.

I6I95 foliosus A. Gray

r6196 ochroleucus A. Gray

I6I97 lynceus Greene

I6I98 Lyoni A. Gray

I6299 MacDougalii Heller

I6200 Macounii Greene

rO20I megacephalus Nutt.

I6202 Memmingeri Britton

I6203 Mendocinensis A. Gray

I6204 microdontus (A. Gray) Heller

I6205 mikanioides Otto

r6206 milleflorus Greene

I6207 Millefolium T. \& G.

I6208 millelobatus Rydb.
I6209 Mohavensis A. Gray

I62 Io multilobatus T. \& G.

16211 mutabilis Greene

I62I 2 Nelsoni Rydb.

I62I3 Uintahensis Aven Nelson

16214 Neo-Mexicanus A. Gray

16215 nephrophyllus Rydb.

I62I6 Newcombei Greene

I62I7 oblanceolatus Rydb.

I62 8 obovatus Muhl.

16219 occidentalis (A. Gray) Greene

16220 rotundatus Rydb.

I622 I Oreganus Howell

I6222 ovinus Greene

I6223 Pagosanus Heller

I6224 palustris (L.) Hook.

16225 Parryi A. Gray

I6226 pauciflorus Pursh

I6227 perennans Aven Nelson

I6228 perezifolius Rydb.

I6229 perplexus Aven Nelson

I6230 petrocallis Greene

(S. petraeus A. Gray)

I623I Plattensis Nutt.

I6232 platylobus Rydb.

I6233 Porteri Greene

I6234 pseudaureus Rydb.

I6235 Pseudo-Arnica Less.

16236 pudicus Greene

I6237 Purshianus Nutt.

16238 rapifolius Nutt.

I6239 rawsonianus Greene

r6240 resedifolius Less.

I624I Columbiensis A. Gray

I6242 Riddellii T. \& G.

I6243 Robbinsii Oakes

I6244 rosulatus Rydb.

I6245 rubricaulis Greene

I6246 Rugelia A. Gray

I6247 Rusbyi Greene

I6248 salicinus Rydb.

r6249 saliens Rydb.

I6250 sanguisorboides Rydb.

I625I scopulinus Greene

16252 Scorzonella Greene

I6253 Scribneri Rydb.

I6254 semiamplexicaulis Rydb.

I6255 serra Hook. 

I6256 integriusculus A. Gray I6257 seridophyllus Greene

I6258 Smallii Britton

I6259 Soldanella A. Gray

I6260 Solidago Rydb.

1626I solitarius Rydb.

I6262 Sonnei Greene

I6263 spatuliformis Heller

I6264 sphaerocephalus Greene

I6265 streptanthifolius Greene

I6266 subcuneatus Rydb.

I6267 subnudus DC.

r6268 subvestitus Howell

I6269 sylvaticus L.

I6270 sylvestris $L$.

1627I taraxacoides (A.Gray) Greene

16272 Thurberi A. Gray

16273 tomentosus Michx.

I6274 Toumeyi Greene

I6275 triangularis Hook.

I6276 Hanseni Greene

I6277 tridenticulatus Rydb.

r6278 trigonophyllus Greene

I6279 valerianella Greene

I6280 variifolius $R y d b$.

I628I viscosus $\mathrm{L}$.

16282 vulgaris $L$.

r6283 Wardii Greene

r6284 werneriaefolius Greene

I6285 Whippleanus A. Gray

I6286 Wootonii Greene

\section{Aretium}

I6287 Lappa L.

r6288 minus Schk.

I6289 tomentosum (Lam.) Schk.

Saussurea

I6290 alpina DC.

I6291 Ledebouri A. Gray

I6292 Americana D. C. Eaton

\section{Carduus}

\section{(Cnicus)}

I6293 altissimus L.

r6294 Americanus (A. Gray) Greene r6295 amplifolius Greene

16296 Andersoni (A. Gray) Greene I6297 Andrewsii (A. Gray) Greene
16298 Arizonicus (A. Gray) Greene

16299 arvensis (L.) Robs.

I6300 Bernardinus Greene

I630I bipinnatus (Eastwood) Heller

I6302 Breweri (A. Gray) Greene

I6303 calcareus (Jones) Heller

I6304 Californicus (A. Gray) Greene

I6305 callilepis Greene

I6306 candidissimus Greene

I6307 canovirens Rydb.

I6308 ciliolatus (Henderson) Heller

I6309 clavatus (Jones) Heller

I63Io crassicaulis Greene

I63I I crispus L.

I6 312 cymosus Greene

I6 ${ }^{1} 3$ diffusus (Eastwood) Heller

I63I4 discolor (Muhl.) Nutt.

I6315 Drummondii (T.\&G.) Coville

I6316 acanlescens (A. Gray)

Coville

I63I7 edulis (Nutt.) Greene

I63I 8 Flodmanii Rydb.

I6319 foliosus Hook.

I6320 fontinalis Greene

I632I Grahami (A. Gray) Greene

16322 Hallii (A. Gray) Heller

I6323 hesperius (Eastwood) Heller

I6324 Hillii (Canby) Porter

r6325 Hookerianus (Nutt.) Heller

16326 hydrophilus Greene

I6327 inamoenus Greene

I6328 Kelseyi Rydb.

I6329 lanceolatus L.

I6330 Lecontei (T. \& G.) Pollard

I633I leiocephalus (D. C. Eaton)

(C. Eatoni A. Gray)

I6332 Mohavensis Greene

I6333 muticus (Michx.) Pers.

I6334 Nebraskensis Britton

I6335 Neo-Mexicanus (A. Gray)

I6336 Nevadensis Greene [Greene

I 6337 nidulus (Jones) Heller

I6 338 nutans L.

I6339 Nuttallii (DC.) Pollard

I6340 occidentalis Nutt.

I634 I ochrocentrus (A.Gray)Greene

I6342 odoratus (Muhl.) Porter

I6343 Parryi (A. Gray) Greene 

16344 perennans Greene

16345 Pitcheri ('Torr.) Porter

16346 Plattensis Porter

I 6347 quercetorum (A.Gray) Greene

16348 remotifolius Hook.

I6349 repandus (Michx.) Pers.

I6350 Rothrockii (A. Gray) Greene

1635I Rusbyi Greene

I6352 scariosus (Nutt.) Heller

I6353 scopulorum Greene

(C. eriocephalus A. Gray)

I6354 spinosissimus Walt.

(C. horridulus Michx.)

I6355 Elliottii ('T. \& G.) Porter

I6356 Tweedyi Rydb.

I6357 undulatus Nutt.

$1635^{8}$ canescens (Nutt.) Porter

16359 Douglasii (DC.) Greene

I6360 megacephalus (A. Gray)

I636I validus Greene

I6362 venustus Greene

I6363 Virginianus L.

I6364. Wheeleri (A. Gray) Heller

I6365 Wrightii (A. Gray) Heller

\section{Cynara}

I6366 cardunculus L. 16367 scolymus L.

\section{Mariana}

(Silybum)

I6368 Mariniana (L.) Hill

\section{Onopordon}

r6369 acanthium L.

\section{Centaurea}

I637o Americana Nutt.

1637 I Calcitrapa L.

I6372 Cyanus L.

I6373 Jacea . L.

I6374 Melitensis L.

I6375 nigra L.

I6376 scabiosa L.

I6377 solstitialis L.

Centrophyllum

r6378 lanatum (L.) DC.

\section{Cnicus}

r6379 benedictus L.

\section{Gochnatia}

I6380 hypolenca A. Gray

Hecastocleis

I638I Shockleyi A. Gray

\section{Thyrsanthema}

(Chaptalia)

I6382 nutans (L.) Kuntze

I6383 Seemani (Sch. Bip.) Kuntze

I6384 seniflosculare (Walt.) Kuntze (C. tomentosa Vent.)

\section{Perezia}

I6385 microcephala A. Gray

I6386 nana A. Gray

I6387 runcinata Lag.

I6388 Thurberi A. Gray

I6389 Wrightii A. Gray

\section{Trixis}

I639o angustifolia DC.

r639I latiuscula A. Gray

\section{CICHORI A CEA E}

\section{Scolymus}

I6392 Hispanicus L.

\section{Cichorium}

I6393 Intybus L.

I6394 divaricatum DC.

\section{Atrichoseris}

I6395 platyphylla A. Gray

\section{Lapsana}

I6396 communis L.

\section{Serinea}

(Apogon)

I6397 gracilis (DC.) Kuntze

I6398 oppositifolia (Raf.) Kuntze

(A. humilis Ell.)

I6399 Wrightii (A. Gray) Kuntze

Phalacroseris

16400 Bolanderi A. Gray

\section{Microseris}

I640I acuminata Greene

I6402 aphantocarpha A. Gray

I6403 attenuata Greene 

I6404 Bigelovii A. Gray

I 6405 Douglasii (DC.) A. Gray

I6406 elegans Greene

I6407 indivisa Greene

I6408 intermedia Greene

16409 Lindleyi A. Gray

164Io linearifolia A. Gray

I64II macrochaeta A. Gray

I64I 2 Parishii Greene

I64I 3 Parryi A. Gray

164I 4 platycarpa A. Gray

I64I 5 tenella (A. Gray) Greene

\section{Scorzonella}

(Microseris in part)

I6416 arguta Drew.

1641 7 Bolanderi (A. Gray) Greene I64I 8 Howellii (A. Gray) Greene 164I9 laciniata Nutt.

r6420 leptosepala Nutt. r642 I maxima Bioletti

I6422 megacephala Greene

I6423 montana Greene

I6424 paludosa Greene

I6425 pratensis Greene

16426 procera (A. Gray) Greene r6427 sylvatica Benth.

\section{Ptilocalais}

(Microseris in part)

I6428 gracililoba (Kellogg) Greene I6429 inajor (A. Gray) Greene I6430 nutans (Geyer) Greene

\section{Nothocalais}

I643I cuspidata (Pursh) Greene

(Troximon cuspidatum Pursh) I6432 nigrescens(Henderson)Heller I6433 Suksdorfii Greene

I6434 troximoides (A. Gray) Greene (Microseris troximoides A. Gray)

\section{Adopogon}

\section{(Krigia)}

I6435 Carolinianum (Walt.) Britton (K. Virginica Willd.)

I6436 Dandelion (L.) Kuntze

16437 montanum (Michx.) Kuntze I6438 occidentale (Nutt.) Kuntze
I6439 Virginicum (L.) Kuntze (K. amplexicaulis Nutt.)

Arnoseris

I6440 minima (L.) Dumort (A. pusilla Gaertn.)

\section{Garhadiolus}

(Rhagadiolus)

r644I hedypnoides (F. \& M.) Saub. \& Spach

\section{Anisocoma}

I6442 acaule T. \& G.

\section{Hypochaeris}

I6443 glabra L.

I6444 radicata $\mathrm{L}$.

\section{Leontodon}

I6445 autumnale L.

I6446 nudicaule (L.) Porter

(L. hirsutum L.)

\section{Picris}

I6447 echioides L.

16448 hieracioides L.

I6449 Japonica Thumb.

\section{Ptiloria}

\section{(Stephanomeria)}

I6450 canescens Greene

I645I cichoriacea (A. Gray) Greene

I6452 coronaria Greene

I6453 divaricata Greene

I6454 exigua (Nutt.) Greene

I6455 filifolia Greene

I6456 lactucina (A. Gray) Greene I6457 lygodesmoides (Jones) Heller I6458 myrioclada (D. C. Eaton)

I6459 Neo-Mexicana Greene

I6460 paniculata (Nutt.) Greene

I646I Parryi (A. Gray) Coville

16462 pauciflora (Torr.) Raf.

(S. runcinata Nutt.)

16463 pentachaeta (D. C. Eaton)

I6464 pleurocarpa Greene [Greene

I6465 ramosa Rydb.

I6466 scabrella Greene

I6467 Schottii (A. Gray) Greene 

I6468 tenuifolia ('Torr.) Raf. (S. minor Nutt.

I6469 Thurberi (A. Gray) Greene I6470 tomentosa Greene I647 I virgata (Benth.) Greene I6472 Wrightii (A. Gray) Greene

\section{Chaetadelpha}

I6473 Wheeleri A. Gray

\section{Nemoseris}

(Rafinesquia)

I6474 Californica (Nutt.) Greene r6475 Neo-Mexicana (A. Gray)

\section{Tragopogon}

I6476 porrifolius L. I6477 pratensis $\mathrm{L}_{4}$.

\section{Pinaropappus}

I6478 roseus Less.

\section{Malacothrix}

16479 altissima Greene i6480 Californica DC.

1648I Clevelandi A. Gray

16482 Coulteri A. Gray 16483 Fendleri A. Gray

I6484 foliosa A. Gray I6485 glabrata (D.C.Eaton) A. Gray I6486 implicata Eastwood I 6487 incana (Nutt.) T. \& G.

I6488 indecora Greene

I6489 insularis Greene

I6490 obtusa Benth.

I649 I runcinata Aven Nelson I6492 saxatilis (Nutt.) T. \& G. I6493 tenuifolia (Nutt.) A. Gray I6494 sonchoides (Nutt.) T. \& G. r6495'squalida Greene I6496 Torreyi A. Gray

\section{Calycoseris}

I6497 Parryi A. Gray I6498 Wrightii A. Gray

\section{Glyptopleura}

I6499 marginata D. C. Eaton r6500 setulosa A. Gray

\section{Chondrilla}

I650r juncea L.

\section{Taraxacum}

I6502 ceratophorum DC.

I6503 eriophorum Rydb.

I6504 erythrospermum Andrz.

I6505 latilobum DC.

(T.officinale var.alpinum of Syn. F1.) I6506 lividum (Wald. \& Kit.)Heller I6507 scopulorum (A. Gray) Rydb. I6508 Taraxacum (L.) Karst.

(T. officinale Weber)

r6509. glaucescens(Koch) Heller

\section{Sonchus}

I65 Io arvensis L.

I65II asper (L.) All.

I 65 I 2 oleraceus L.

I65I3 tenerrimus L.

\section{Lactuca}

I65I4 campestris Greene

I65I 5 Canadensis L.

I65I6 Floridana (L.) Gaertn.

r6517 graminifolia Michx.

I65I 8 hirsuta Muhl.

I6519 Ludoviciana (Nutt.) DC.

I6520 Morsii Robinson

I652I multifida Rydb.

I6522 pulchella (Pursh) DC.

I6523 sagittifolia Ell.

(L. integrifolia Bigel.)

I6524 Scariola L.

16525 spicata (Lam.) A. S. Hitchc.

(L. leucophaea A. Gray)

I6526 integrifolia (A. Gray)

Britton

I6527 sylvatica Aven Nelson I6528 villosa Jacq.

(L. acuminata A. Gray)

\section{Apargidium}

I6529 boreale T. \& G.

\section{Lygodesmia}

I6530 aphylla DC.

1653 I Texana T. \& G.

I6532 exigua A. Gray

I6533 grandiflora T. \& G. 
I 6534 juncea (Pursh) D. Don

I6535 rostrata A. Gray

I6536 spinosa Nutt.

I6537 lanata Henderson

\section{Agoseris}

(Troximon in part)

I $653^{8}$ alpestris (A. Gray) Greene I6539 altissima Rydb.

I6540 apargioides (Less.) Greene

1654I Arizonica Greene

I 6542 aurantiaca (Hook.) Greene

I6543 barbellulata Greene

I6544 carnea Rydb.

I6545 dasycarpa Greene

I6546 dens-leonis Greene

I6547 elata (Nutt.) Greene

(T. Nuttallii A. Gray)

I6548 glauca (Pursh) Greene

I6549 gracilens (A. Gray) Greene

I6550 graminifolia Greene

I655I Greenei (A. Gray) Rydb.

I6552 grandiflora (Nutt.) Greene

I6553 heterophylla (Nutt.) Greene

I6554 cryptopleura Greene

I6555 kymapleura Greene

16556 hirsuta (Hook.) Greene

( $T$. humile A. Gray)

I6557 intermedia Greene

I6558 laciniata (Nutt.) Greene

I6559 Leontodon Rydb.

(T. glaucum var. laciniatum A.Gray) I6560 aspera Rydb.

I656I pygmaea Rydb.

I6562 major Jepson

I6563 Marshallii Greene

I6564 montana Osterhout

I6565 monticola Greene

I6566 parviflora (Nutt.) Dietr.

16567 plebeia Greene

r6568 pumila (Nutt.) Rydb.

I6569 purpurea Greene

I6570 retrorsa (Benth.) Greene

I657 I rosea (Nutt.) Dietr.

I6572 scorzoneraefolia (Schrad.)

I6573 villosa Rydb.

\section{Sitilias}

(Pyrrhopappus)

I6574 Caroliniana (Walt.) Raf.

I6575 grandiflora (Nutt.) Greene (P. scaposus DC.)

I6576 multicaulis (DC.) Greene

I6577 Rothrockii (A. Gray) Greene

\section{Crepis}

I6578 acuminata Nutt.

I6579 Andersoni A. Gray

I6580 atribarba Heller

I658 $\mathrm{I}$ barbiger Leiberg

I6582 Bakeri Greene

16583 biennis $\mathrm{L}$.

I6584 elegans Hook.

16585 glauca (Nutt.) T. \& G.

I6586 gracilis (D. C. Eaton) Rydb.

r6587 grandifolia Greene

I6588 intermedia A. Gray

I6589 pleurocarpa A. Gray

I6590 lancifolia Greene

r659I Modocensis Greene

I6592 monticola Coville

I6593 nana Richards.

I6594 occidentalis Nutt.

I6595 costata A. Gray

I6596 crinita A. Gray

I6597 Nevadensis Kellogg

r6598 platyphylla Greene

I6599 pulchra L.

I6600 pumila Rydb.

I660r riparia Aven Nelson

I6602 rostrata Coville

16603 runcinata (James) T. \& G.

I6604 alpicola Rydb.

I6605 ciliosa Greene

I6606 hispidulosa Howell

r6607 scopulorum Coville

I6608 subacaulis (Kellogg) Coville

I6609 subcaruosa Greene

I66Io tectorum L.

I66 I I virens $\mathrm{L}$.

\section{Nabalus}

(Prenanthes)

r66 2 albus (L.) Hook. 

I66r 3 altissimus (L.) Hook.

I66I4 asper (Michx.) T. \& G. 166r 5 barbatus ('T. \& G.) Heller I66I6 Boottii DC.

I66I 7 crepidineus (Michx.) DC. I66I 8 hastatus (Less.) Heller (P. alata A. Gray)

I66I9 Mainensis (A. Gray) Heller I6620 nanus (Bigel.) DC.

I662I racemosus (Michx.) DC. I6622 pinnatifida(A.Gray)Britton r6623 sagittatus (A. Gray) Rydb. I6624 Serpentaria (Pursh) Hook. I6625 integrifolius(Cass.)Britton I6626 trifoliolatus Cass. 16627 virgatus (Michx.) DC.

\section{Hieracium}

r6628 albiflorum Hook. I6629 amplum Greene I6630 argutum Nutt. I663I argyraeum Small I6632 aurantiacum L. I6633 barbigerum Greene r6634 Bolanderi A. Gray I6635 Brandegei Greene I6636 Canadense Michx. I6637 carneum Greene r6638 cynoglossoides Arvet I6639 nudicaule A. Gray I6640 Fendleri Sch. Bip. I664I discolor A. Gray
16642 Mogollense A. Gray I6643 gracile Hook.

I6644 detonsum A. Gray I6645 minimum Aven Nelson

I6646 Greenei A. Gray

I6647 Greenii Porter \& Britton I6648 griseum Rydb.

16649 Gronovii L.

I6650 horridum Fries

r665I Howellii A. Gray

I6652 Lemmoni A. Gray

I6653 longipilum Torr.

I6654 Marianum Willd.

r6655 megacephalon Nash

r6656 murorum L.

r6657 nigrocollinum Wats.

I6658 paniculatum L.

I6659 Parishii A. Gray

I666o Pilosella L.

r666I Peleterianum Mer.

I6662 praealtum Vill.

I6663 pratense Tausch.

I6664 Pringlei A. Gray

r6665 Rusbyi Greene

I6666 Wrightii A. Gray

I6667 scabrum Michx.

r6668 Scouleri Hook.

I6669 Scribneri Small

r667o triste Cham.

I667 I umbellatum L.

I.6672 venosum $\mathrm{L}$.

I6673 vulgatum Fries 



\section{ADDITIONS AND CORRECTIONS}

l'age 13

274a var. Sonnei Henderson

I95a occidentalis Henderson

Page 17

542 read (Micheli) not (Michx.)

Page 18

623 read Halepense

Page 49

2898a longifolium Osterhout 2917 a rubrum Osterhout

Page 50

2988a Masseyi C. W. Hyams

Page 54

Sisyrinchium 1\%8\%

Strike ont 3300 and 3308

Page 55

\section{Hydastylus (128\%)}

(Sisyrinchium in part)

335 oa borealis Bicknell

335 ob brachypus Bicknell

335 oc Californicus (Ker) Salisb. (S. Californicum Ait. f.)

335od Elmeri (Greene) Bicknell (S. Elmeri Greene)

335 oe longipes Bicknell 335 of rivularis Bicknell

Page 56

3424 a constricta Small 3431a Reverchoni Small 3434a triloba Small

Page 58

$354 \mathrm{I}$ instead of $334 \mathrm{I}$

3554a bella Piper

Page 59

3653a subcoertlea Piper

Page 63

3898 a inequalis Stokes
Page 64

3998 and 3999, not 4098 and 4099

Page 73

4707a Tetonensis Elias Nelson

Page 78

5017 laciniata

Page 79

5roza Treleasei Bush

Page 8I

$5217 \mathrm{a}$ var. laetus Greene

5236 a var. Deforesti Davis

526ra McCallai Davis

5286 pedatifidus

Page 86

559ra var. papilliferum Henderson 5638 a perplexum Henderson 5638 bubicarpum Aven Nelson

Page 90

5866a Yellowstonensis Aven Nelson

592 ra densicaulis Aven Nelson

$5922 a$ divaricarpa Aven Nelson

5924 a elegans Aven Nelson

5927a fruticosa Aven Nelson

Page 9I

5943a lignipes Aven Nelson

595ra pendulocarpa Aven Nelson

Page 94

6r 34 (Coult. \& Fisher) Coult.

6r 58a cognata Elias Nelson

Page 95

6256 var. Oregonensis

6258a saxicola Elias Nelson

Page 97

6384 a saximontana Elias Nelson

Page 104

6892a grosse-serrata Elias Nelson 
Page ro5

6946 , not 6046

Page 108

723ra var. Tetonensis Elias Nelson

Page 109

7286 a ramosus Elias Nelson

Page II 2

7547 , not 7247

Page II 5

7738 a var. Weiserensis Jones

7778 a Craigi Jones

Page I 30

8882 phyllanthoides

Before 8904, insert

\section{Acer}

Page I 35

9259 Thombifolia L.

Page 136

9304 smilacifolia

Page 138

$945^{8}$, not $64^{8} 8$

Page I 39

9515a Thorii Aven Nelson

Page I4I

9687 var. Neo-Mexicanus

9694, not 6694

Page 142

973I, not 7931

Page 145

9943a Wyomingense Aven Nelson 9988a strigosa Rydb.

9988b subulata Rydb.

ro002a gypsophila(Eastwood)Heller

Page 146

I0045a filifolia (Eastwood) Wooton

Page 154

10573a var. glandifera Porter

Page I 55

Strike out 10667 , which $=10703$
Page I 58

I 0826 mastichodendron

Page I 59

rog20a decandra (Walt.) Harper $=$ I0922, which strike out

Page 167

I I 5 II, not I25II

Page 168

I 594 a inconspicua Henderson

I $596 \mathrm{a}$ Kirtleyi Henderson

Page I 73

I 1924a var. flexuosa Aven Nelson I 1934 cycloptera

I $1952 \mathrm{a}$ multicaulis Aven Nelson

Page 174

I 2001a amoena Aven Nelson

Page 183

I 2698a caudatus Heller

Page 184

I 2798 radicosus Aven Nelson

Page 185

I 2836 tomentosa ('Thunb.) Baill

Page 187

r 30 ro scabra

Page 188

I3II8 stricta Rydb.

Page 193

\section{Galium}

I 343 Ia ambiguum W. F. Wright I 3446 a chartaceum W. F. Wright r 3449a Coloradoense W. F. Wrigh Page 194

I3465a muricatum W. F. Wright I 3475 a siccatum W. F. Wright $13475 \mathrm{~b}$ sparsiflorum W. F. Wrigh I 3476 a subscabridum W. F. Wrigh I 3480 a var. diversifolium

I 3483 a var. submontanum

W. F. Wrigh

W. F. Wrigh 
Page 201

I 396ra erecta Aven Nelson

I 399 1a subincisa Greene

I 3993 a divergens Greene

I 3993b diversifolia Greene

I 3994a fasciculata Greene

I 3994b filifolia Greene

I 3994c glomerella Greene

I 3994d juncea Greene

I 3994e lepidota Greene

I 3994 f longifolia Greene

I 3997a serotina Greene

I 3998a tenuis Greene

Page 202

I 4062a asperula Desf.

I 4063a bellidifolia Greene

Page 203

r4I rga Maxoni Pollard

I4I38a pallescens Mohr

I 4I45a pruinosa Greene

I4i72a subviscosa Greene

14I84a var. calcicola Fernald

Page 204

I4196a var. petiolaris Aven Nelson

I4223a Hallii (A. Gray) Howell
Page 207

r 4474 a var. graminifolius Baker

Page 208

r 4498a var. pallidus Cockerell

r 4499a var. viridis Cockerell

I452 ra Latahensis Henderson

I 455 Oa var. exiguus Fernald

I4573a var. cinerascens Fernald

Page 209

I $45^{89}$ a var. compactus Fernald

I $4589 \mathrm{~b}$ var. demissus Lindl.

I459 Ia var. oligocephalus Fernald

I 462 Ia var. vestitus Fernald

I 4637 a var. saxatilis Fernald

Page 212

I 4890 consanguinea DC.

Page 229

I6085 comosa A. Gray

Page 230

I6199, not 16299

I620I, not IO2OI 



\section{INDEX}

\begin{tabular}{|c|c|c|c|c|c|}
\hline Abama & 47 & Ailanthus & 124 & Aneimia & 9 \\
\hline Abies & 15 & Aira & 26 & Anemiopsis & 57 \\
\hline Abronia & 71 & Ajuga. & 175 & Anemone & 79 \\
\hline Abutilon & 133 & Albizzia & 105 & Anemone & 80 \\
\hline Acacia & 105 & Alchemilla & 104 & Anemonella & So \\
\hline Acaena & 104 & Aletes & 150 & Angelica & I5I \\
\hline Acalypha & I 26 & Aletris & 52 & Anhalonium & 141 \\
\hline Acamptopappus & 201 & Aligera & I95 & Anidrum & 149 \\
\hline Acanthochiton & 69 & Alisma & 17 & Anisacanthus & I9I \\
\hline Acanthomintha & 177 & Alliaria & 86 & Anisocarpus & 220 \\
\hline Acanthoscyphus & 63 & Allionia & 70 & Anisocoma & 233 \\
\hline Acanthospermum & 215 & Allionia & $7 \mathrm{I}$ & Annona & 77 \\
\hline Acer & 130 & Allium & 48 & Anoda & 135 \\
\hline Acerates & 163 & Allocarya & 171 & Anogra & 145 \\
\hline Achillea & 226 & Allotropa & I 53 & Anredera & 70 \\
\hline Achlys & 82 & Alnus & 60 & Antennaria. & 213 \\
\hline Achyrachaena & 222 & Alopecurus & 24 & Anthaenantia & 19 \\
\hline Acleisanthes & 71 & Alophia & 54 & Anthemis & 226 \\
\hline Acnida & 69 & Alsine & 74 & Anthephora & 19 \\
\hline Aconitum & 79 & Alternanthera & 70 & Anthericum & $4^{8}$ \\
\hline Acorus & 44 & Althaea & ' 34 & Anthochloa & 29 \\
\hline Achroanthes & $5^{6}$ & Alyssum & 91 & Anthoxanthum & 22 \\
\hline Achyronichia & 76 & Amaranthus & 69 & Anthriscus & 148 \\
\hline Acrostichum & I 2 & Amblyolepis & 225 & Antirrhinum & 182 \\
\hline Actaea & 78 & Amblyopappus & 224 & Anychia & 76 \\
\hline Actinella & 224,225 & Ambrosia & 215 & Apargidium & 234 \\
\hline Actinolepis & 224 & Amelanchier & 99 & Apera & 26 \\
\hline Actinomeris & 218 & Amianthemum & 47 & Aphanisma & 67 \\
\hline Actinospermum & 220 & Ammannia & 142 & Aphanostephus & 206 \\
\hline Acuan & 106 & Ammobroma & 153 & Aphyllon & 190 \\
\hline Adelia & I 59 & Ammodenia & 76 & Apiastrum & 149 \\
\hline Adenocaulon & $2 \mathrm{I} 4$ & Ammophila & 26 & Apios & $12 \mathrm{I}$ \\
\hline Adenostegia & I 88 & Ammoselinum & 149 & Apium & 149 \\
\hline Adenostoma & 103 & Amoreuxia & 138 & Aplectrum & 57 \\
\hline Adiantum & I 2 & Amorpha & II 2 & Aplopappus 204. 205, & 210 \\
\hline Adicea & 62 & Ampelanus & 163 & Apocynum & 161 \\
\hline Adopogon & 223 & Ampelopsis & 133 & Apodanthera & I96 \\
\hline Adlumia & 84 & Amphiachyris & 201 & Apodanthes & 63 \\
\hline Adolphia & 132 & Amphianthus & 186 & Apogon & 232 \\
\hline Adonis & 82 & Amphicarpaea & 121 & Apteria & 55 \\
\hline Adoxa & 195 & Amphicarpon & 19 & Aquilegia & 78 \\
\hline Aegopodium & 150 & Amsinckia & 17.3 & Arabis & 90 \\
\hline Aegopogon & 19 & Amsonia & 161 & Arabis & $9 !$ \\
\hline Aeschynomene & 119 & Amygdalus & 105 & Aragallus & If \\
\hline Aesculus & I $3 \mathrm{I}$ & Amyris & 124 & Aralia & 148 \\
\hline Aethusa & I 50 & Anagallis & 157 & Arbutus & I55 \\
\hline Afzelia & 187 & Anamomis & 143 & Arceuthobium & 62 \\
\hline Agastache & 176 & Anantherix & 163 & Arctagrostis & 25 \\
\hline Agave & 53 & Anaphalis & 214 & Arcteranthis & 82 \\
\hline Ageratum & 198 & Ancistrocarphus & 213 & Arctium & $23 !$ \\
\hline Agoseris & 235 & Andrachne & 125 & Arctomecon & 84 \\
\hline Agrimonia & 104 & Andromeda & 155 & Arctostaphylos & 155 \\
\hline Agropyron & 33 & Andropogon & 18 & Ardisia & ${ }^{156}$ \\
\hline Agrostemma & 73 & Androsace & 157 & Arenaria & 75 \\
\hline Agrostis & 25 & Androstephium & 49 & Arenaria & 76 \\
\hline
\end{tabular}


Arethusa

Argentina

Argyrothammia $\quad 126$

Arisaema

Aristida

Aristolochia

Argemone

Arnica

Arnoseris

Aronia

Arrhenatherum

Artemisia

Aruncus

Arundinaria

Arundo

Asarum

Asclepias

Asclepias

Asclepiodora

Ascyrum

Asimina

Asparagus

Asperugo

Asperula

Aspicarpa

Aspidium

Asplenium

Asprella

Astephanus

Aster

Aster

Astilbe

Astragalus I14, II 8, II9

Astrophyllum I $\quad$ I 24

Ataenia

Atamisquea

Atamosco

Athysanus

Atragene

Atrichoseris

Atriplex

Audibertia

Avena

Avicennia

Ayenia

Azalea

Azaleastrum

Azolla

$\begin{array}{lr}\text { Baccharis } & 212 \\ \text { Baeria } & 222 \\ \text { Baeria } & 223 \\ \text { Bahia } & 224 \\ \text { Baileya } & 222 \\ \text { Baldrinia } & 220 \\ \text { Ballota } & 177 \\ \text { Balsamorrhiza } & 217 \\ \text { Baptisia } & 107 \\ \text { Barbarea } & 87 \\ \text { Bartlettiat } & 228 \\ \text { Bartonia } & 160 \\ \text { Bartsia } & 189 \\ \text { Batis } & 70 \\ \text { Batodendron } & 156 \\ \text { Batrachium } & 82 \\ \text { Bebbia } & 220\end{array}$

Beckmannia

Beckwithia

Bejaria

Belamacanda

Bellivia

Bellis

Beloperone

Benzoin

Berberis

Berchemia

Bergia

Berginia

Berlandiera

Bernardia

Berteroa

Berula

Betonica

Betula

Bicuculla

Bidens

Bifora

Bigelovia

Bignonia

Biolettia

Blechnum

Blennosperma

Blepharoneuron

Blepharipappus

Blepharipappus

Blepharizonia

Blephilia

Bletia

Blitum

Bloomeria

Bocconia

Boehmeria

Boerhaavia

Boisduvalia

Bolandra

Bolelia

Boltonia

Borago

Borreria

Borrichia

Boschniakia

Botrychium

Bouchea

Bouchetia

Bourdonia

Bourreria

Bouteloua

Bouvardia

Bowlesia

Boykinia

Brachyactis

Brachychaeta

Brachyelytrum

Bradburya

Bradburya

Brandegea

Brasenia

Brassica

Brauneria

Braya

Brazoria

Brevoortia

\section{8}

So

154

54

18 I

206

192

$\mathrm{S}_{3}$

82

I31

137

191

215

126

9 I

150

177

59

84

219

149

205,206

190

223

II

225

25

22 I

220

$22 \mathrm{I}$

I 78

57

68

49

84

62

71

145

93

198

206

173

193

217

190

9

175

182

206

171

27

193

I 48

94

2 IO

204

24

I 2 I

201

196

77

87

217

91

176

50

Breweria

Brickellia

Brintonia

Brittonamra

Brittonastrum

Briza

Brodiaea

Bromus

Brongniartia

Broussonetia

Brunnichia

Bryanthus

Bryophyllum

Buchloe

Buchnera

Buckleya

Buda

Buddleia

Bulbilis

Bumelia

Bupleurum

Burmannia

Burrielia

Bursa

Bursera

Butneria

Byrsonimia

64

200

203

I 14

I 76

30

49

32

I 3

61

6;

I 54

93

28

I 88

62

76

I 59

28

$15^{8}$

149

55

222

89

125

$8_{3}$

I 24

Cabomba $\quad 77$

Cacalia

Cacaliopsis

229

Cactus

Caesalpinia

Cakile

Colamagrostis

Calamagrostis

Calamintha

Calamovilfa

Calandrinia

Calceolaria

Calla

Calliandra

Callicarpa

Callirhoe

Callitriche

Calluna

Calochortus

Calophanes

Calopogon

Caltha

Calycadenia

Calycanthus

Calycocarpum

Calycoseris

Calydora

Calypso

Calyptranthes

Calyptridium

Calyptrocarpus

228

I $4 \mathrm{I}$

I07

87

25

26

I 78

26

72

I 38

44

IO5

175

134

I 28

156

50

I9I

57

77

221

83

$\mathrm{S}_{3}$ 


\begin{tabular}{|c|c|c|c|c|c|}
\hline Campyloneuron & 12 & Cercidium & 107 & Citharexylum & 175 \\
\hline Canavalia & 122 & Cercis & 106 & Citrullus & 196 \\
\hline Canella & 138 & Cercocarpus & 103 & Citrus & 124 \\
\hline Canbya & $3_{4}$ & Cereus & 140 & Cladium & 37 \\
\hline Canna & 55 & Cestrum & 182 & Cladothamnus & I54 \\
\hline Cannabis & 62 & Cevallia & 139 & Cladothrix & 70 \\
\hline Canotia & 130 & Chaenactis & 224 & Cladrastis & Iu7 \\
\hline Capnoides & $\$_{4}$ & Chaerophyllum & $14^{8}$ & Clappia & 222 \\
\hline Capnorea & 170 & Chaetadelpha & 234 & Clarkia & 145 \\
\hline Capparis & 92 & Chaetocalyx & 119 & Claytonia & 72 \\
\hline Capraria & 186 & Chaetochloa & 21 & Clematis & So \\
\hline Capriola & 27 & Chaetopappa & 206 & Cleome & 92 \\
\hline Capsella & 89 & Chamaebatia & 103 & Cleomella & 92 \\
\hline Capsicum & 181 & Chamaebatiaria & 98 & Clethra & 153 \\
\hline Cardamine & 87 & Chamaecistus & 154 & Cliftonia & 129 \\
\hline Cardiospermum & 131 & Chamaecrista & 106 & Clinopodium & 173 \\
\hline Carduus & $23 I$ & Chamaecyparis & I 5 & Clintonia & $5 I$ \\
\hline Carex & $3^{8}$ & Chamaedaphne & 155 & Clitoria & 121 \\
\hline Carica & 139 & Chamaelirion & 47 & Clusia & 1 36 \\
\hline Carlowrightia & 191 & Chamaenirion & 145 & Cneoridium & 124 \\
\hline Carminatia & 199 & Chamaerhodos & 103 & Cnicus & 232 \\
\hline Carpenteria & 96 & Chamaesaracha & 180 & Cnicus & 231 \\
\hline Carpephorus & 200 & Chapmania & I 19 & Coccoloba & 67 \\
\hline Carphochaete & 199 & Chaptalia & 232 & Coccolobis & 67 \\
\hline Carpinus & 59 & Cheilanthes & I I & Coccothrinax & 43 \\
\hline Carum & 150 & Cheilogramma & 12 & Cocculus & $8_{3}$ \\
\hline Carya & 57 & Cheiranthus & 91 & Cochlearia & 86 \\
\hline Cassandra & I 55 & Cheiroglossa & 9 & Cochlearia & 90 \\
\hline Cassia & 106 & Chelidonium & 84 & Cochranea & 171 \\
\hline Cassiope & I 54 & Chelone & ${ }_{1} 8_{3}$ & Cocos & 44 \\
\hline Cassytha & 83 & Chenopodium & 67 & Coelopleurum & I 51 \\
\hline Castalia & 77 & Chilopsis & 190 & Coix & is \\
\hline Castanea & 60 & Chimaphila & 153 & Coldenia & 171 \\
\hline Castanopsis & 60 & Chiococca & 193 & Coleanthus & 24 \\
\hline Castela & I 24 & Chiogenes & I 55 & Coleogyne & 103 \\
\hline Castilleja & I 88 & Chionanthus & I 59 & Colesanthus & 200 \\
\hline Casuarina & 57 & Chionophila & 185 & Collinsia & 182 \\
\hline Catabrosa & 29 & Chloris & 27 & Collinsonia & ISo \\
\hline Catalpa & 190 & Chlorogalunı & 48 & Collomia & 166 \\
\hline Catesbaea & 193 & Choisya & 124 & Cologania & I 21 \\
\hline Cathestecum & $2 S$ & Chondrilla & 234 & Colpodium & - 31 \\
\hline Catopsis & 45 & Chondrophora & 206 & Coloptera & 151 \\
\hline Cancalis & I 49 & Chorizanthe & 63 & Colubrina & 132 \\
\hline Caulanthus & 85 & Chrosperma & 47 & Comandra & 62 \\
\hline Caulophyllum & $\mathrm{S}_{2}$ & Chrysactinia & 226 & Comarella & 103 \\
\hline Cayaponia & 196 & Chrysamphora & 92 & Comarum & 102 \\
\hline Ceanothus & 132 & Chrysanthemum & 226 & Commelina & 45 \\
\hline Cebatha & $\mathrm{s}_{3}$ & Chrysaspis & III & Comptonia & $5^{8}$ \\
\hline Cedronella & 176 & Chrysobolanus & 105 & Conanthus & 170 \\
\hline Celastrus & 130 & Chrysogonum & 215 & Condalia & 131 \\
\hline Celosia & 69 & Chrysoma & 204 & Conioselinum & I 51 \\
\hline Celtis & 61 & Chrysophyllum & $15^{8}$ & Conium & 149 \\
\hline Cenchrus & 22 & Chrysopogon & 18 & Conobea & 186 \\
\hline Centaurea & 232 & Chrysopsis & 201 & Conocarpus & 143 \\
\hline Centella & 148 & Chrysosplenium & 96 & Conopholis & Igu \\
\hline Centromadia & 221 & Chrysothamnus & 205 & Conradina & 179 \\
\hline Centrophyllum & 232 & Chylisma & 147 & Conringia & $9^{2}$ \\
\hline Centrosema & 121 & Chytraculia & 143 & Convallaria & 52 \\
\hline Centunculus & $15 i$ & Cicer & 120 & Convolvulus & 164 \\
\hline Cephalanthera & 56 & Cichorium & 232 & Conzya & 212 \\
\hline Cephalanthus & 193 & Cicuta & 149 & Cooperia & 53 \\
\hline Ceranthera & 179 & Cienfugosia & 136 & Coptis & 78 \\
\hline Cerastium & 74 & Cimicifuga & 78 & Corallorhiza & 56 \\
\hline Ceratiola & 129 & Cinna & 25 & Corchorus & 133 \\
\hline Ceratophyllum & 77 & Circaea & 147 & Cordia & 170 \\
\hline Ceratopteris & 12 & Cissus & 1.3 .3 & Cordylanthus & 185 \\
\hline
\end{tabular}




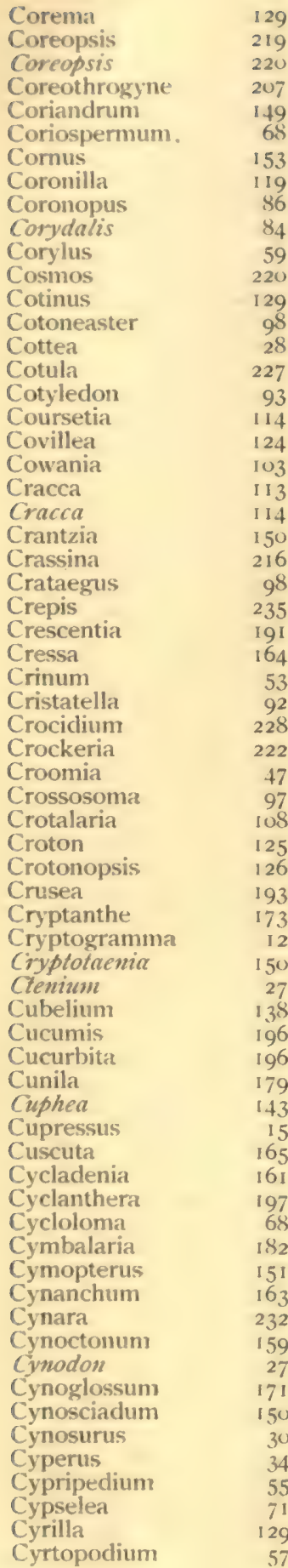

Cyrtorhyncha

Discopleura

150

Cystopteris

Cytisus

Disporum

52

10

Dissanthelium

Distichlis

Dactylis 30

Dactyloctenium

Ditaxis

126

Dalea

Dithyrea

Dalbergia

Dodecatheon

Dalibarda

Damasonium

Dodonaea

Doellingeria

I 3 I

Panthonia

Dolicholus

209

Daphne

Darbya

Dolichos

122

Dondia

Douglasia

Darlingtonia

Doreningia

Dasiophora

Dasylirion

Draba

Draba

Dasystoma

Dracocephalum

Datisca

Datura

Daubentonia

Draperia

Drosera

Daucus

Dryas

Decodon

Decumaria

Deinandra

Delphinium

Dendrium

Dendromecon

Dendrophylax

Dennstaedtia

Dentaria

Drymaria

Drymocallis

Dryopetalon

Dryopteris

Drypetes

Duchesnea

Dugaldea

Dulichium

Dupatya

Deringa

Deschampsia

Dupontia

I 22

Desmanthus

Duranta

Dysodia

Desmodium

Eastwoodia

Diamorpha

Dianthera

Dianthus

Diapedium

Diapensia

Diarrhena

Dicentra

Dichaetophora

Dichondra

Dichromena

Dicksonia

Dicliptera

I 19

93

I9I

Dicoria

Dicranocarpus

Dicraurus

Eatonella

Eatonia

Ecastophyllum

Echinacea

Echinocactus

Echinocereus

Echinochloa

Echinodorus

Echinopanax

Echinopepon

Echinospermum

Echites

205

Didiplis

Diervilla

Digitalis

Dimeresia

Echiun

Eclipta

Edwinia

222

29

120

140

140

140

Ehretia

Eichhornia

Elatine

Elaeagnus

Eleocharis

Dioclea

Diodia

Elephantella

Dionaea

Elephantopus

Dioscorea

Diospyros

Eleusine

Elionurus

Elliottia

Dipetalia

Dipholis

Diphylleia

Ellisia

Elodea

Diplachne

Elnya

Diplacus

Diplotaxis

Elytraria

Elscholtzia

Dipsacus

Elymus

Dirca

Emmenanthe 


\begin{tabular}{|c|c|c|c|c|c|}
\hline Emorya & I 59 & Evax & 213 & Gemmingia & 54 \\
\hline Empetrum & 129 & Evolvulus & 164 & Genipa & 193 \\
\hline Encelia & 218 & Exothea & I 31 & Genista & 109 \\
\hline Engelmannia & 215 & Exostema & I93 & Gentiana & 160 \\
\hline Enslenia & 163 & Eysenhardtia & 112 & Geranium & I 22 \\
\hline Ephedra & I5 & & & Gerardia & 187 \\
\hline Epicampes & 25 & Fagonia & 124 & Geum & 103 \\
\hline Epidendrum & 57 & Fagopyrum & 67 & Gibbesia & 76 \\
\hline Epicaea & I 55 & Fagus & 60 & -Gilia & 166 \\
\hline Epilobium & 144 & Falcata & 121 & Gilia & 167,168 \\
\hline Epilobium & 145 & Fallugia & 103 & Gillenia. & 98 \\
\hline Epipactis & 56 & Fatsia & 147 & Githopsis & 197 \\
\hline Epiphegus & 190 & Fendlera & 96 & Glaucium & 84 \\
\hline Equisetum & 12 & Fendlerella & 96 & Glaux & 157 \\
\hline Eragrostis & 29 & Festuca & 31 & Glecoma & 176 \\
\hline Eranthis & 78 & Ficaria & 82 & Gleditsia & 107 \\
\hline Erechtites & 228 & Ficus & $6 r$ & Glinus & 71 \\
\hline Eremiastrum & 207 & Filago & 213 & Glossopetalon & 130 \\
\hline Ereminula & 214 & Filipendula & IO4 & Glottidium & II 4 \\
\hline Eremocarpus & I 26 & Filix & Io & Glyceria & $3^{I}$ \\
\hline Eremocarya & 172 & Fimbristylis & 37 & Glycosma & 149 \\
\hline Eremocrinum & 48 & Fistularia & 190 & Glycyrrhiza & II \\
\hline Erianthus & is & Flaveria & 223 & Glyptopleura & 234 \\
\hline Erica & I 56 & Floerkea & 129 & Gnaphalium & 214 \\
\hline Erigenia & 149 & Florestina & 223 & Gnaphalodes & 213 \\
\hline Erigeron & 210 & Flourensia & 218 & Gochnatia & 232 \\
\hline Erigeron & 212 & Foeniculum & 150 & Godetia & 145 \\
\hline Eriocarpum & 204 & Forestiera & 159 & Gomphocarpus & 162 \\
\hline Eriocaulon & 45 & Forsellesia & 130 & Gomphrena & 70 \\
\hline Eriochloa & 19 & Fothergilla & 97 & Gonolobus & 163 \\
\hline Eriocoma & 23 & Fouquiera & 137 & Goodyera & 56 \\
\hline Eriodictyon & 170 & Fragaria & 100 & Gordonia & ז 36 \\
\hline Eriogonum & 64 & Frankenia & I 37 & Gossypianthus & 71 \\
\hline Eriogynia & 98 & Franseria & 216 & Gossypium & I 36 \\
\hline Eriophorum & 36 & Frasera & I6I & Gouania & 132 \\
\hline Eriophyllum & 223 & Fraxinus & I 58 & Graphephorum & $3 I$ \\
\hline Eriophyllum & 224 & Fremontodendron & I 36 & Gratiola & I 86 \\
\hline Erithalis & 193 & Fremontia & I 36 & Grayia & 68 \\
\hline Eritrichiun & 171 & Fritillaria & 50 & Greggia & 91 \\
\hline Erodium & 123 & Froelichia & 70 & Greenella & 206 \\
\hline Emodea & 193 & Fugosia & I36 & Grindelia & 201 \\
\hline Ervum & $12 \mathrm{I}$ & Fuirena & 36 & Guajacum & 124 \\
\hline Eryngium & 148 & Fumaria & 84 & Guardiola & 215 \\
\hline Erysimum & $9 \mathrm{I}$ & & & Guettarda & .93 \\
\hline Erythraea & 160 & Gaertneria & 216 & Guilandina & 107 \\
\hline Erythrina & I2I & Gaillardia & 225 & Guilleminea & 69 \\
\hline Erythronium & 50 & Galactia & I2I & Gutierrezia & 201 \\
\hline Eschscholtzia & 83 & Galax & I 56 & Gyminda & I 30 \\
\hline Eucephalus & 210 & Galeopsis & 177 & Gymnanthes & 127 \\
\hline Eucharidium & I 45 & Galinsoga & 220 & Gymnocladus & 107 \\
\hline Eucnide & 140 & Galium & . 193 & Gymnogramma & II \\
\hline Eucrypta & 168 & Galphimia & 123 & Gymnolomia & 217 \\
\hline Eugenia & 143 & Galpinsia & 146 & Gymnopteris & II \\
\hline Eulobus & 146 & Galvesia & 182 & Gymnosperma & 201 \\
\hline Eulophus & 150 & Garberia & 200 & Gymnosteris & 167 \\
\hline Eunanus & 186 & Garhadiolus & 233 & Gymnopogon & 27 \\
\hline Euonymus & 130 & Garrya & 153 & Gypsophila & 74 \\
\hline Eupatorium & 199 & Gastridium & 25 & Gyrostachys & 56 \\
\hline Euphorbia & 127 & Gatesia & 192 & Gyrotheca & 53 \\
\hline Euphrasia & 189 & Gaultheria & I 55 & & \\
\hline Eurotia. & 68 & Gaura & 147 & Habenaria & 55 \\
\hline Euthamia & 203 & Gaurella & I 46 & Hackelochloa & 18 \\
\hline Eurytaenia & 150 & Gaylussacia & 155 & Halenia & 161 \\
\hline Eustachys & 27 & Gayophytum & 147 & Halesia & 158 \\
\hline Fustoma & 161 & Gelasine & 54 & Halophila & 17 \\
\hline Eutrema & 86 & Gelsimium & I 59 & Halophyton & 161 \\
\hline
\end{tabular}




\begin{tabular}{|c|c|c|c|c|c|c|}
\hline Hanamelis & 97 & Hipponane & 127 & Iresine & & 70 \\
\hline Hamelia & 193 & Hippurus & 147 & & & 53 \\
\hline Haploesthes & 228 & Hoftmanseggia & 107 & Isanthus & & 175 \\
\hline Haplophyton & 161 & Hofmeisteria & 199 & Isnardia & & 144 \\
\hline Harbouria & 149 & Holacantha & 124 & Isocarpha & & 216 \\
\hline Harpaecarphus & 221 & Holcus & 26 & Isocoma & & 206 \\
\hline Harpagonella & 174 & Hollisteria & 63 & Isoetes & & 13 \\
\hline Hartmannia & 146 & Holocarpha & 221 & Isomeris & & $9^{2}$ \\
\hline Hartwrightia & $19^{8}$ & Holodiscus & $9^{8}$ & Isopappus & & 205 \\
\hline Hastingsia & 48 & Holosteum & 75 & Isopyrum & & -8 \\
\hline Hazardia & 206 & Holozonia & 221 & Itea & & $9^{6}$ \\
\hline Hecastocleis & 232 & Homalobus & II 8 & Iva. & & 215 \\
\hline Hechtia & 45 & Hookera & 49 & Ivesia & 102, & , 103 \\
\hline Hedeoma & 178 & Homalocenchrus & 22 & Ixophorus & & 21 \\
\hline Hedera & 147 & Hordeum & 33 & & & \\
\hline Hediomoides & 179 & Horkelia. & 102 & Jacobinia & & 191 \\
\hline Hedysarum & 119 & Horsfordia & 133 & Jacquemontia & & 164 \\
\hline Heimia & 143 & Hosackia & III & Jacquinia & & 156 \\
\hline Heleastrum & 209 & Hottonia & 157 & Jamesia & & 96 \\
\hline Heleniastrum & 225 & Houstonia & 192 & Janusia & & 123 \\
\hline Helenium & 225 & Houttuynia & 57 & Jasione & & 197 \\
\hline Heleochloa & 24 & Howellia & 198 & Jatropha & & 126 \\
\hline Helianthella & 218 & Hudsonia & I 37 & Jaumea & & 222 \\
\hline Helianthemum & 137 & Hulsea & 224 & Jeffersonia & & S2 \\
\hline Helianthus & 217 & Humulus & $6 \mathrm{I}$ & Jepsonia & & 95 \\
\hline Helietta & 124 & Hutchinsia & 89 & Juglans & & 57 \\
\hline Heliopsis & 216 & Hydastylus & 55,237 & Juncoides & & 47 \\
\hline Heliotropiunı & 171 & Hydrangea & 96 & Juncus & & 46 \\
\hline Helleborus & 78 & Hydrastis & 77 & Juniperus & & 15 \\
\hline Helonias & 47 & Hydrochloa & 22 & Jussiaea & & 144 \\
\hline Hemerocallis & 48 & Hydrocotyle & 148 & Justicia & & 191 \\
\hline Hemicarpha & 34 & Hydrolea & 170 & & & \\
\hline Hemieva & 94 & Hydrophyllum & 168 & Kalmia & & I 54 \\
\hline Hemitomes & 154 & Hygrophíla & 191 & Kallstroemia & & 124 \\
\hline Hemizonia & $22 \mathrm{I}$ & Hymenatherum & 226 & Karwinskia & & I3I \\
\hline Hemizonella & 221 & Hymenocallis & 53 & Keerlia & & 206 \\
\hline Hepatica & 79 & Hymenoclea & 215 & Kelloggia & & 193 \\
\hline Heracleum & 152 & Hymenopappus & 223 & Kelseya & & 98 \\
\hline Hermannia & 136 & Hymenothryx & 223 & Kickxia & & 182 \\
\hline Hermidium & 70 & Hyoscyamus & 180 & Knautia & & 196 \\
\hline Herpestis & 186 & Hypelate & I3I & Kneiffia & & 146 \\
\hline Hesperalcea & I 35 & Hypericum & I 37 & Kobresia' & & 38 \\
\hline Hesperaloe & $5 \mathrm{I}$ & Hypochaeris & 223 & Kochia & & 68 \\
\hline Hesperalaea & 159 & Hypopitys & 153 & Koeberlinia & & 138 \\
\hline Hesperevax & 213 & Hypoxis & 53 & Koeleria & & 29 \\
\hline Hesperis & 91 & Hyptis & 180 & Koellia & & 179 \\
\hline Hesperocallis & 48 & Hyssopus & 179 & Koeniga & & 63 \\
\hline Hesperochiron & 170 & Hystrix & 34 & Koenig & & 91 \\
\hline $\begin{array}{l}\text { Hesperocnide } \\
\text { Hesperogenia }\end{array}$ & 62 & & & Korycarpus & & 30 \\
\hline Hesperogenia & 149 & $\begin{array}{l}\text { Ibervillea } \\
\text { Icacora }\end{array}$ & 196 & Kosteletzkya & & ${ }^{1} 36$ \\
\hline Heteranthera & 46 & Icacora & 156 & Krameria & & 106 \\
\hline Heterocodon & 197 & Ichthyomethia & 120 & Kraunhia & & II 4 \\
\hline Heterodraba & 90 & Ilex & 129 & Krigia & & 233 \\
\hline Heterogaura & 147 & Ilicioides & 130 & Krynitzkia I & 171,172 & 173 \\
\hline Heteromeles & 99 & Illicium & 77 & Kuhnia & & 200 \\
\hline Heteropogon & 19 & Ilysanthes & 186 & Kuhnistera & & 113 \\
\hline Heterospermum & 219 & Impatiens & 131 & Kunzia & & 103 \\
\hline Heterotheca & 201 & Imperatoria & I 52 & Kyllinga & & 35 \\
\hline Heuchera & 95 & Imperata & 18 & & & \\
\hline Hexalectris & 57 & Indigofera & 112 & Lachnanthes & & 53 \\
\hline Hibiscus & 135,136 & Ingenhouzia & I 36 & Lachnocaulon & & 45 \\
\hline Hicoria & 57 & Inula & 214 & Lachnostoma & & 163 \\
\hline Hieracium & 236 & Iodanthus & $9^{1}$ & Lacinaria & & 200 \\
\hline Hierochloe & 22 & Ionactis & 209 & Lactuca & & 234 \\
\hline Hilaria & 19 & Ionidium & 138 & Lagenaria & & 196 \\
\hline Hippocratea & 130 & Ipomoea & 164 & Lagerstroemia & & 14.3 \\
\hline
\end{tabular}


Lagophylla

Lagotis

Laguncularia

Lamarckia

Lamium

Langloisia

Lantana

Laphamia

Laportea

Lappula

Lapsana

Larrea

Larix

Lastarriaea

Lasthenia

Lathyrus

Laurentia.

Lavatera

Lavauxia

Layia

Leavenworthia

Lechea

Ledum

Leersia

Leibergia

Leiophyllum

Leitneria

Legouzia

Lembertia

Lemmonia

Lemna

Leonotis

Leontodon

Leonurus

Lepachys

Lepargyraea

Lepidium

Lepidospartium

Leptamnium

Leptandra

Leptarrhena

Leptaxis

Leptilon

Leptocaulis

Leptochloa

Leptoglossis

Leptorchis

Leptosyne

Leptotaenia

Lepturus

Lepuropetalon

Lespedeza

Lesquerella

Lessingia

Leucaena

Leucampyx

Leucelene

Leucocrinum

Leucophyllum

Leucophysalis

Leucosyris

Leucothoe

Lewisia

Lewisia

Liatris

Libocedrus
Ligusticum

Ligustrum

Lilaea

Lilaeopsis

Lilium

Limnanthemum

Limnanthes

Limnobium

Limnodia

Limodorum

Limonium

Limosella

Linanthus

Linaria

Lindera

lindheimera

Linnaea

Linum

Liparis

Lipocarpha

Lippia

Liquidambar

Liriodendron

Listera

Lithophila

Lithophragma

Lithospermum

Litsea

Littorella

Lloydia

Lobelia

Lochnera

Loeflingia

Loeselia

Loiseleuria

Lolium

Lomaria

Lonicera

Lophanthus

Lophiola

Lophophora

Lophotocarpus

Lotus

Ludwigia

Ludwigiantha

Luffa

Luina

Lunaria

Lupinus

Lutkea

Luziola

Lychnis

Lychnis

Lycium

Lycopersicon

Lycopodium

Lycopsis

Lycopus

Lycurus

Lygodesmia

Lygodium

Lyonothamnus

Lyrocarpa

Lysichiton

Lysiloma

Lysimachia

\section{Lythrum}

Macbridea

Macdougalia

Machaeranthera

Maclura

Macounastrum

Macranthera

Macrocalyx

Macronema

Macrosiphonia

Madaria

Madia

Madia

Magnolia

Maianthemume

Mairania

Malachra

Malacothrix

Malapoenna

Malpighia

Malus

Malva

Malvastrum

Malvaviscus

Mammilaria

Manihot

Manisurus 


\begin{tabular}{|c|c|c|c|c|c|}
\hline Meriolix & 146 & Myginda & 130 & Oligomeris & 92 \\
\hline Mertensia & 174 & Myosotis & 173 & Olneya & 114 \\
\hline Mesadenial & 229 & Myosurus & 80 & Omphalodes & 171 \\
\hline Mesembrianthemum & 71 & Myrica & 57 & Onagra & 145 \\
\hline Mesosphaerum & 180 & Myrica & $5^{3}$ & Onobrychis & II \\
\hline Metastelma & 163 & Myriophyllum & 147 & Onoclea & Iu \\
\hline Michelliella & ISO & Myrsine & 156 & Onopordon & 232 \\
\hline Micrampelis & 196 & & & Onosmodium & 174 \\
\hline Micranthemum & 186 & Nabalus & 235 & Oonopsis & 204 \\
\hline Microcala & 159 & Nacrea & 214 & Ophioglossum & 9 \\
\hline Micromeria & 178 & Naias & I7 & Oplismenus & 21 \\
\hline Micropus & 213 & Nama & 170 & Opulaster & 97 \\
\hline Microrhamnus & $13 \mathrm{I}$ & Napaea & 135 & Opuntis & 141 \\
\hline Microseris & 232 & Nardus & 33 & Orchis & 55 \\
\hline Wicroseris & 233 & Narthecium & 47 & Orcuttia & $2 S$ \\
\hline Microsteris & 166 & Nasturtium & 87 & Oreastrum & 210 \\
\hline Microstylis & 56 & Naumburgia & 157 & Oreobroma & 72 \\
\hline Mikania & 199 & Navarretia & 167 & Oreocarya & 172 \\
\hline Milium & 23 & Nazia & 19 & Oreodoxa & 44 \\
\hline Milla & 50 & Nectandra & 83 & Oreoxis & $15 \mathrm{I}$ \\
\hline Mimetanthe & 185 & Neillia & 97 & Origanum & I 79 \\
\hline Mimosa & 105 & Nelumbo. & 77 & Ornithogalum & $5 I$ \\
\hline Mimulus & 185 & Nemacaulis & I3 & Ornithopteris & 9 \\
\hline Mimulas & 186 & Nemacladus & 197 & Orobanche & $190^{\circ}$ \\
\hline Mimusops & 158 & Nemastylis & 54 & Orochaenactis & 22.4 \\
\hline Mirabilis & 70 & Nemopanthes & 130 & Orogenia & 149 \\
\hline Mitchella & I93 & Nemophila & 168 & Orontium & 44 \\
\hline Mitella & 95 & Nemoseris & 234 & Orophaca & I 8 \\
\hline Mitella & 96 & Neowashingtonia & 43 & Orthocarpus & 189 \\
\hline Mitellastra & 96 & Nepeta & 176 & Oryctes & 180 \\
\hline Mitracarpus & I 93 & Nephrolepis & Io & Oryzopsis & 23 \\
\hline Mitreola & I 59 & Nephropetalum & 136 & Osbertia & 205 \\
\hline Modiola & I 34 & Nephrophyllidium & $16 I$ & Osmanthus & I 59 \\
\hline Moehringia & 76 & Neptunía & 106 & Osmaronia & 104 \\
\hline Moenchia & 75 & Nerium & 162 & Osmorrhiza & $I 48,149$ \\
\hline Mogiphanes & 70 & Nesaea & 143 & Osmunda & 9 \\
\hline Mohavea & 182 & Neslia & 89 & Ostrya & 59 \\
\hline Mohrodendron & 158 & Nesodraba & 90 & Oxalis & 123 \\
\hline Molinia & 29 & Nestronia & 62 & Oxybaphus & 70 \\
\hline Molucella & 180 & Neviusia & 99 & Oxycoccus & 56 \\
\hline Mollugo & $7 \mathrm{I}$ & Newberrya & I 54 & Oxydendron & 155 \\
\hline Momordica & 196 & Nicandra & I8o & Oxygraphis & 80 \\
\hline Monanthochloe & 28 & Nicolletia & 225 & Oxypolis & I5I \\
\hline Monarda & 178 & Nicotiana & 182 & Oxyria & 66 \\
\hline Monardella & 179 & Nissolia & 119 & Oxystylis & $9^{2}$ \\
\hline Monerma & 33 & Nitrophila & 67 & Oxytenia & 215 \\
\hline Moneses & I 53 & Nolina & $5 \mathrm{I}$ & Oxytheca & 63 \\
\hline Monniera & I 86 & Nothocalais & 233 & Oxytria & $4^{8}$ \\
\hline Monnina & 125 & Notholaena & II & Oxytropis & in 8 \\
\hline Monolepis & 68 & Nothoscordum & 49 & & \\
\hline Monoptilon & 206 & Nuphar. & 77 & Pachylophus & 146 \\
\hline Monolopia & 223 & Nuttallia. & 104 & Pachysandra & I 29 \\
\hline Monotropa & I53 & Nyctaginia & 70 & Pachystima & 130 \\
\hline Monotropsis & I 53 & Nymphaea & 77 & Paeonia & 77 \\
\hline Montia & 72 & Nyssa & I 53 & Paeplanthus & 45 \\
\hline Morinda & I93 & & & Palafoxia & 223 \\
\hline Morongia & 105 & Oakesia & 48 & Palmerella & 198 \\
\hline Mortonia & I 30 & Obolaria & 160 & Panax & 148 \\
\hline Morus & 61 & Ocimum & I 80 & Panicularia & $3^{1}$ \\
\hline Muhlenbergia & 23 & Ocotea & 83 & Panicum & 19 \\
\hline Muilla & 49 & Odontites & 189 & Panicum & $2 \mathrm{I}$ \\
\hline Munroa & 28 & Odontostomum & 48 & Papaver & 84 \\
\hline Muscari & $5 \mathrm{I}$ & Oenanthe & 150 & Pappophorum & 28 \\
\hline Musenion & 149 & Oenothera & 145 & Parietaria & 62 \\
\hline Museniopsis & 149 & Oenothera 145, 146, & 147 & Parishella & I97 \\
\hline Myagrum & 87 & Oldenlandia & 192 & Paritium & I 36 \\
\hline
\end{tabular}




\begin{tabular}{|c|c|c|c|c|c|}
\hline Parkinsonia & 107 & Philotria & 17 & Podostemon & 93 \\
\hline Parnassia & 96 & Philoxerus & 70 & Podostigma & 162 \\
\hline Paronychia & 76 & Phippsia & 24 & Pogogyne & 179 \\
\hline Parosela & I 12 & Phlebodium & 12 & Pogonia & 55 \\
\hline Parrasia & $9 \mathrm{I}$ & Phleum & 24 & Polanisia & 92 \\
\hline Parrya & 91 & Phlomis & 176 & Polemonium & 168 \\
\hline Parryella & I 12 & Phlox & 165 & Poliomintha & 178 \\
\hline Parsonsia & 143 & Phoenicaulis & 92 & Polycarpon & 76 \\
\hline Parthenice & 215 & Pholisma & 154 & Polycodium & I56 \\
\hline Parthenium & 215 & Phoradendron & 62 & Polygala & I 25 \\
\hline Parthenocissus & 133 & Phragmites & 28 & Polygonatum & 52 \\
\hline Paspalum & 19 & Phryma & 192 & Polygonella & 67 \\
\hline Passiflora & 139 & Phyla & 175 & Polygonum & 66 \\
\hline Pastinaca & 152 & Phyllanthus & I 25 & Polymeia & 214 \\
\hline Pattalias & 163 & Phyllitis & 10 & Polypodium & 12 \\
\hline Paulownia & 185 & Phyllodice & 154 & Polypogon & 25 \\
\hline Pavonia & I 35 & Phyllospadix & 16 & Polypremum & 159 \\
\hline Pectis & 226 & Phymatodes & 12 & Polypteris & 223 \\
\hline Pectocarya & 171 & Physalis & I 80 & Polystachya & 57 \\
\hline Pedicularis & 189 & Physalis & $\mathrm{I} 8 \mathrm{I}$ & Polystichum & Io \\
\hline Pedicularis & 190 & Physalodes & I 80 & Polytaenia & $15 \mathrm{I}$ \\
\hline Peganum & I 24 & Physaria & 88 & Pontederia & 45 \\
\hline Pelargonium & 123 & Physocarpus & 97 & Ponthieva & 56 \\
\hline Pellaea & II & Physostegia & I 76 & Populus & $5^{8}$ \\
\hline Peltandra & 44 & Physurus & 56 & Porliera & I 24 \\
\hline Peltiphyllum & 95 & Phytolacca & 70 & Porophyllum & 226 \\
\hline Pennisetum & 22 & Piaropus & 45 & Porteranthus & 98 \\
\hline Pentacaena & 77 & Picea & I4 & Porterella & 198 \\
\hline Pentachaeta & 201 & Pickeringia & 108 & Portulaca & 72 \\
\hline Penthorum & 93 & Picradenia & 225 & Potamogeton & 16 \\
\hline Pentodon & 192 & Picramnia & 24 & Potentilla & 100 \\
\hline Pentstemon & I8 3 & Picris & 233 & Potentilla & $\mathrm{O} 2, \mathrm{IO}_{3}$ \\
\hline Pentstemon & 185 & Pieris & I 55 & Poterium & 104 \\
\hline Peperomia & 57 & Pilea & 62 & Prenanthes & 235 \\
\hline Peramium & 56 & Pilularia & I 2 & Primula & I 56 \\
\hline Peraphyllum & 99 & Pimpinella & 150 & Prionopsis & 204 \\
\hline Perezia & 232 & Pinaropappus & 234 & Priva & 175 \\
\hline Pericome & 222 & Pinckneya & 192 & Proserpinaca & 147 \\
\hline Perilla & 180 & Pinguicula & 190 & Prosopis & 106 \\
\hline Periploca & 162 & Pinus & 14 & Prunella & I 76 \\
\hline Perityle & 222 & Piptocalyx & 172 & Prunus & $\mathrm{IO}_{4}$ \\
\hline Persea & $8_{3}$ & Piriqueta & 139 & Psaracha & I $8 \mathrm{i}$ \\
\hline Petalonyx & 139 & Piscidia & 120 & Psathyrotes & 228 \\
\hline Petalostemon & I13 & Pisonia & 71 & Pseudocymopterus & I5I \\
\hline Petasites & 228 & Pistia & 44 & Pseudophoenix & 44 \\
\hline Peteria & 114 & Pithecolobium & I05 & Pseudotsuga & I5 \\
\hline Petiveria & 70 & Plagiobothrys & 172 & Psidium & 143 \\
\hline Petradoria & 204 & Planera & 61 & Psilactis & 206 \\
\hline Petrophyton & 98 & Plantago & 192 & Psilocarphus & 213 \\
\hline Petroselinum & 149 & Platanus & 97 & Psilocarya & 37 \\
\hline Petunia & 182 & Platyspermum & 88 & Psilostrophe & 222 \\
\hline Peucedanum & I 52 & Platystemon & 83 & Psilotum & 13 \\
\hline Peucephyllum & 228 & Platystigma & 83 & Psoralea. & 112 \\
\hline Phaca & 118 & Plectritis & 195 & Psychotria & 193 \\
\hline Phacelia & 169 & Pleea & 47 & Ptelea & 124 \\
\hline Phalacroseris & 232 & Pleuricospora & 154 & Pteridium & 12 \\
\hline Phalaris & 22 & Pleurogyne & 160 & Pteris & 12 \\
\hline Phanerophlebia & so & Pleuropogon & 30 & Pterocaulon & 213 \\
\hline Pharus & 22 & Pluchea & 213 & Pterospora & 153 \\
\hline Phaseolus & 122 & Plumbago & 158 & Pterostegia & 63 \\
\hline Phegopteris & Io & Plummera & 225 & Ptilimnium & 150 \\
\hline Phellopterus & 151 & Pneumaria & 174 & Ptilocalais & 233 \\
\hline Pherotrichus & 163 & Poa & 30 & Ptilonella & 220 \\
\hline Philadelphus & 96 & Podistera & 150 & Ptiloria & 233 \\
\hline Philibertella & 162 & Podophyllum & 82 & Puccinellia & 31 \\
\hline Philibertia & 162 & Podostemma & 163 & Pulsatilla & 80 \\
\hline
\end{tabular}




\begin{tabular}{|c|c|c|c|c|c|}
\hline Punica. & 143 & Ruppia & 16 & Scoparia & 186 \\
\hline Purpusia & 103 & Rydbergia & 225 & Scorzonella & 23.3 \\
\hline Purshia & 103 & & & Scribneria & 33 \\
\hline Pycnanthemum & 179 & Sabal. & 43 & Scrophularia & 183 \\
\hline Pyrola & 153 & Sabbatia & 159 & Scutellaria & 175 \\
\hline Pyrrhopappus & 235 & Sageretia & 131 & Sebastiania & 127 \\
\hline Pyrrocoma & 204 & Sagina & 75 & Secondatia & $16 i$ \\
\hline Pyrularia & 62 & Sagittaria & 17 & Sedum & 93 \\
\hline Pyrus & 99 & Salicornia & 69 & Selaginella & $\begin{array}{l}93 \\
13\end{array}$ \\
\hline \multirow[t]{2}{*}{ Pyxidanthera } & 156 & Salix & $5^{8}$ & Selenia & 88 \\
\hline & & Salizaria & 175 & Selinocarpus & \\
\hline Quamasia & 51 & Salpichroa & 181 & Selinum & 150 \\
\hline Quamoclit & 164 & Salsola & 69 & Sempervium & \\
\hline Quercus & 60 & Salvia & 177 & Senebiera & 86 \\
\hline \multirow[t]{2}{*}{ Quincula } & I8I & Salviastrum & 178 & Senecio & 229 \\
\hline & & Salvinia & I 2 & Sereneum & 217 \\
\hline Rafinesquia & 234 & Sambucus & 194 & Serenoa & 44 \\
\hline Raillardella & 228 & Samolus & 157 & Sericocarpus & 212 \\
\hline Raineria & 228 & Sanicula & 148 & Serinea & 232 \\
\hline Ramona & 178 & Sanguinaria & 84 & Serjania & 131 \\
\hline Randia & 193 & Sanguisorba & 104 & Sesamum & 191 \\
\hline Ranunculus & so & Sanvitalia & 216 & Sesban & 114 \\
\hline Ranunculus & so, 82 & Sapindus & I 31 & Sesbania & 114 \\
\hline Raphanus & 87 & Sapium. & 127 & Sesuvium & $7 i$ \\
\hline Rapistrnm & 87 & Sąponaria & 77 & Sequoia & 15 \\
\hline Ratibida & 217 & Sarcobatus & 69 & Setaria & 21 \\
\hline Razoumofskya & 62 & Sarcodes & 153 & Seymeaia & 187 \\
\hline Redfieldia & 28 & Sarothra & I 37 & Shepherdia & 142 \\
\hline Reimeria & 19 & Sarracenia & 92 & Sherardia & 193 \\
\hline Reseda & 92 & Sartwellia & 223 & Shortia & 156 \\
\hline Reverchonia & 125 & Sassafras & $\mathrm{s}_{3}$ & Sibara & 38 \\
\hline Reynosia & 131 & Satureia & 178 & Sibbaldia & 103 \\
\hline Rhagadiolus & 233 & Saururus & 57 & Sibbaldiopsis & 103 \\
\hline Rhamnidium & I3I & Saussurea & 231 & Sicyos & 196 \\
\hline Rhamnus & I3I & Savastana & 22 & Sicyosperma & 197 \\
\hline Rhaphidophyllum & 43 & Saxifraga & 94 & Sida & I 35 \\
\hline Rhexia & 143 & Saxifraga & 94.95 & Sidalcea & 134 \\
\hline Rhinanthus & 190 & Saxifragopsis & 95 & Sidalcea & 135 \\
\hline Rhizophora & 143 & Scabiosa & 196 & Sideroxylon & 158 \\
\hline Rhododendron & 154 & Scaevola & 198 & Sieglingia & 28 \\
\hline Rhodora & 154 & Scandix & 148 & Sieversia & 103 \\
\hline Rhus & 129 & Schaefferia & 130 & Silene & 73 \\
\hline Khynchosia & 122 & Schedonnardus & 27 & Silphium & 215 \\
\hline Rhynchospora & 37 & Scheuchzeria & 17 & Silybum & 232 \\
\hline Ribes & 96 & Schinus & 129 & Simarouba & 124 \\
\hline Richardia & 193 & Schizaea & 9 & Simondsia & 129 \\
\hline Ricinella & 126 & Schizandra & 77 & Sinapis & 87 \\
\hline Ricinus & 126 & Schizonotus & 162 & Siphonoglossa & 191 \\
\hline Riddellia & 222 & Schkuhria & 223 & Siphonychia & 76 \\
\hline Rigiopappus & 224 & Schoenocaulon & 51 & Sisymbrium & 0 \\
\hline Rivina & 70 & Schoenocrambe & 87 & Sisymbrium & 86,90 \\
\hline Robinia & I 44 & Schoenolirion & 48 & Sisyrinchium & 54 \\
\hline Romanzoftia & 170 & Schoepfia & 62 & Sitanion & 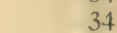 \\
\hline Romneya & $8_{3}$ & Schrankia & 105 & Sitilias & 235 \\
\hline Roripa & 87 & Schwalbea & 190 & Sium & 150 \\
\hline Rosá & 104 & Schweinitzia & 153 & Smelowskia & 90 \\
\hline Rotala & 144 & Scirpus & 36 & Smilacina & 52 \\
\hline Rothrockia & 163 & Scleranthus & 76 & Smilax & \\
\hline Rottboellia & IS & Scleria & 38 & Solanum & ISI \\
\hline Roubieva & 67 & Sclerocarpus & 216 & Solea & ${ }^{1} 38$ \\
\hline Roulinia & 163 & Sclerolepis & 198 & Solidago & 202 \\
\hline Rubus & 99 & Scleropogon & 28 & Solidago & $20,3,204$ \\
\hline Rubus & iox) & Scoliopus & 52 & Soliva & 227 \\
\hline Rudbeckia & 216 & Scolochloa & 31 & Sonchus & 234 \\
\hline Ruellia & I9I & Scolopendrium & 10 & Sonnea & 172 \\
\hline Rumex & 66 & Scolymus & 232 & Sophia & \\
\hline
\end{tabular}




\begin{tabular}{|c|c|c|c|c|c|}
\hline Sophora & 107 & Suckleya & 68 & Tiarella & 95 \\
\hline Sorbus & 99 & Suksdorfia & 94 & Tiedemannia & 151 \\
\hline Sorghum & 18 & Sullivantia & 94 & Tigridia & 54 \\
\hline Spathyema & 44 & Suriana & 12.4 & Tilia & 133 \\
\hline Sparganium & 15 & Swertia & 160 & Tillaea & 93 \\
\hline Spartina & 17 & Swietenia & 125 & Tillandsia & 45 \\
\hline Specularia & 197 & Sympetaleia & 140 & Tinantia & 45 \\
\hline Spergula & 76 & Symphoricarpos & 194 & Tipularia & 57 \\
\hline Spergularia & 76 & Symphytum & 173 & Tissa & 76 \\
\hline Spermacoce & 193 & Symplocarpus & +4 & Tithonia & 217 \\
\hline Spermolepis & 149 & Symplocos & 158 & Tofieldia & 47 \\
\hline Sphacele & 178 & Synandra & 176 & Tolmiea & 95 \\
\hline Sphaeralcea. & 133 & Synedrella & 219 & Tonella & 183 \\
\hline Sphaeromeria & 227 & Syndesmon & 80 & Torreya & 14 \\
\hline Sphaerostigma & 146 & Synosma & 229 & Tournefortia & I 71 \\
\hline Sphenoclea & 197 & Syntherisma & 21 & Townsendia & 206 \\
\hline Spiesia & IIS & Synthlipsis & 88 & Toxylon & 61 \\
\hline Spigelia & 159 & Synthyris & 187 & Trachelospermun & 161 \\
\hline Spilanthes & 218 & Syntrichopappus & 223 & Trachypogon & 18 \\
\hline Spiraea & 98 & Syringa & 159 & Tradescantia & 45 \\
\hline Spiraea & 98,104 & & & Tragia & 126 \\
\hline Spiranthes & 56 & Taeniopleurum & 150 & Tragopogon & 234 \\
\hline Spirodela & 44 & Taenidia & 150 & Tragus & 19 \\
\hline Spirostachys & 69 & Taenitis & 12 & Trapa & 147 \\
\hline Sporobolus & 24 & Tagetes & 225 & Trautvetteria & 80 \\
\hline Sporobolus & 25 & Talinopsis & 72 & Treleasea & 45 \\
\hline Spraguea & 71 & Talinum & 72 & Trema & 61 \\
\hline Stachys & 177 & Tamarix & 137 & Trepocarpus & 149 \\
\hline Stachytarpheta & 175 & Tanacetum & 225,226 & Triadenum & 136 \\
\hline Stanfordia & 92 & Taraxacum & 234 & Trianthema & 71 \\
\hline Stanleya & 85 & Taraxia & 146 & Tribulus & I 24 \\
\hline Stapfia & 29 & Taxodium & 15 & Tricardia & 170 \\
\hline Staphylea & 130 & Taxus & $\mathrm{I} 4$ & Trichloris & 27 \\
\hline Statice & 158 & Tecoma & 191 & Trichocoronis & 199 \\
\hline Steironema & 157 & Tectaria & 10 & Trichoptilium & 224 \\
\hline Stellaria & 74 & Telanthera & 70 & Trichostema & 175 \\
\hline Stellariopsis & 103 & Tellima & 95 & Trichomanes & 9 \\
\hline Stemodia & I 86 & Tephrosia & I1 3 & Tricuspis & 28 \\
\hline Stenandrium & I9I & Terminalia & 143 & Trientalis & 157 \\
\hline Stenanthium & 47 & Tetraclea & I 75 & Trifolium & I09, II I \\
\hline Stenolobium & 191 & Tetradymia & 229 & Triglochin & 27 \\
\hline Stenophragma & 90 & Tetragonanthus & I6I & Trilisa & 199 \\
\hline Stenophyllus & 37 & Tetragonotheca & 216 & Trillium & 52 \\
\hline Stennorhynchus & 56 & Tetramerium & 191 & Triodia & 28 \\
\hline Stenosiphon & 147 & Tetraneuris & 224 & Triosteum & 194 \\
\hline Stenotaphrum & 22 & Teucrium & 175 & Triplasis & 28 \\
\hline Stenotus & 205 & Thalictrum & $8_{2}$ & Tripsacum & 18 \\
\hline Stephanomeria & 233 & Thalesia & 190 & Trisetum & 26 \\
\hline Stevia & 198 & Thalia & 55 & Tritelia & 49 \\
\hline Stillingia & 127 & Thamnosma & 124 & Triumfetta & 133 \\
\hline Stipa & 23 & Thaspium & I5I & Trixis & 232 \\
\hline Stipulicida & 76 & Thelesperma & 219 & Trollius & 78 \\
\hline Stokesia & 198 & Thelypodium & 85 & Tropaeolum & 123 \\
\hline Streptanthus & $8_{5}$ & Thermopsis & 107 & Tropidocarpum & 88 \\
\hline Streptopus & 53 & Therofon & 94 & Troximon & 233,235 \\
\hline Stropholirion & 50 & Thespesia & 136 & Tsuga & 14 \\
\hline Strophostyles & 122 & Thlaspi & 86 & Tubiflora & 191 \\
\hline Strumpfia & 193 & Thrinax & +3 & Tumion & 14 \\
\hline Struthiopteris & II & Thryallis & 123 & Tunica & 74 \\
\hline Stuartia & I 36 & Thuja & 15 & Turnera & I 39 \\
\hline Stylocline & 213 & Thurberia & 25 & Tussilago & 228 \\
\hline Stylophorum & 84 & Thurovia & 206 & Typha & 15 \\
\hline Stylosanthes & 119 & Thymophylla & 226 & & \\
\hline Styrax & 158 & Thymus & 179 & Ulex & 109 \\
\hline Suaeda & 69 & Thyrsanthema & 232 & Ulmaria & 104 \\
\hline Subularia & 86 & Thysanocarpus & 90 & Ulmus & $6 I$ \\
\hline
\end{tabular}




\begin{tabular}{|c|c|c|c|c|c|}
\hline Limbellularia & 83 & Viburnum & 194 & Xanthisma & 201 \\
\hline Incinia & 38 & Vicia & 120 & Xanthium & 216 \\
\hline Ungmadia & 131 & Vigna & 122 & Xanthocephalum & 201 \\
\hline [nifolium & 52 & Viguiera & 217 & Xanthorrhiza & 78 \\
\hline Iniola & 30 & Vinca & $16 \mathrm{I}$ & Xanthosma & 44 \\
\hline Urechites & 161 & Vincetoxicun & 163 & Xerophyllum & 47 \\
\hline Urena & 135 & Viola & 138 & Ximenia & 62 \\
\hline L'rtica & 62 & Vitex & 175 & Xolisma & I55 \\
\hline Urticastrum & 62 & Vitis & 132 & Xylorrhiza & $2 \mathrm{I}(\mathrm{C})$ \\
\hline Urvillea & 131 & Vittaria & I 2 & Xylothermia & 108 \\
\hline Utricularia & 190 & Viscaria & 73 & Xyris & 44 \\
\hline \multirow[t]{2}{*}{ Uvularia } & \multirow{2}{*}{48} & Vleckia & 176 & & \\
\hline & & Voyria & 161 & Yeatesia & 192 \\
\hline Vaccaria & 74 & & & Yucca & $5 \mathrm{I}$ \\
\hline Vaccinium & 155,156 & Waldsteinia & 103 & & \\
\hline Vagnera & 52 & Waltheria & 136 & Zaluzania & 217 \\
\hline Valeriana & 195 & Warea & 84 & Zamia & I4 \\
\hline Valerianella & 195 & Washingtonia & 43,148 & Zannichellia & 17 \\
\hline Vallesia & 161 & Websteria & 34 & Zanthoxylum & 124 \\
\hline Vallisneria & 18 & Wedelia & 71,217 & Zauschneria & I 44 \\
\hline Vanclevea & 201 & Whipplea & 96 & Zenobia & I 55 \\
\hline Vancouveria & 82 & Whitnea & 222 & Zephyranthes & 53 \\
\hline Vanilla & 56 & Willughbaea & 199 & Zexmenia & 219 \\
\hline Varilla & 216 & Wislizenia & 92 & Zinnia & 216 \\
\hline Vauquelinia & 98 & Wissadula & 133 & Zizania & 22 \\
\hline Velaea & 149 & Wisteria & II 4 & Zizaniopsis & 22 \\
\hline Velezia & 74 & Wolffia & 44 & Zizia & 149 \\
\hline Venegasia & 222 & Wolffiella & 44 & Zizyphus & 131 \\
\hline Veratrum & 48 & Woodsia & 9 & Zonanthemis & 221 \\
\hline Verbascum & 182 & Woodwardia & II & Zornia & II9 \\
\hline Verbena & 174 & Wootonia & 220 & Zostera & 16 \\
\hline Verbesina & $2 \mathrm{IS}$ & Wulfenia & 187 & Zygadenus & 47 \\
\hline Veronica & 187 & Wyethia & 217 & Zygia. & 105 \\
\hline Vernonia & 198 & Wyomingia & 212 & Zygophyllum & I 24 \\
\hline
\end{tabular}




\section{THE BOTANICAL GAZETTE}

Eidited by John M. COul'ter, Head Professor of Botany in the University of Chicago. Published monthly, with illustrations. Subscription price, $\$ 4.00$ a year in the United States; foreign, $\$ 4.50$; single copies, 50 cents.

The Botanical Gazette is an illustrated monthly journal devoted to botany in its widest sense. For more than fwenty years it has been the representative American journal of botany, containing contributions from the leading botanists of America and Europe. In addition to the formal papers presenting the results of research, current information and discussion are given in the editorials, and in the departments of Current Literature, Open Letters, Notes for Students, and Notes and News.

\section{Representative Comment}

"One of the best journals of its kind now published."

B. T. Galloway, U. S. Dept. of Agriculture.

"It is simply indispensable to the botanist, teacher or student."

W. A. Kellerman, Ohio State University.

"It is a credit to American botany. In its present form it has increased claims upon the support of botanists."

George L. Goodale, Harvard University.

" It well represents the progress of botanical science in the United States.'

Douglas $H$. Campbell, Leland Stanford University.

Send subscriptions to

\section{THE UNIVERSITY OF CHICAGO PRESS, Chicago, III.}

\section{SILENE 2490 \\ Caryophyllaceae \\ 62}

You can obtain genus labels like the above for $\mathbf{8 1 . 0 0}$ a thousand. The numbers, taken from the Genera Siphonogamarum of Torre \& Harms, show the systematic arrangement of the genera in Engler \& Prantl's Naturlichen Pfanzenfamilien. The number 62 represents the position of the genus in the family Caryophyllaceae, and 2490 the total number of the genus in the sequence. The genera represented in this Catalogue will be printed in black ink, foreign genera in red ink. Either series may be had separately. Several labels will be furnished for large genera. Send your order to

\section{A. A. HELLER,}




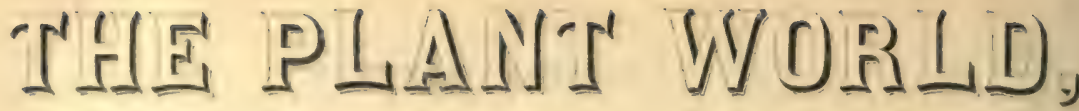

An Illustrated Monthly Journal of Popular Botany,

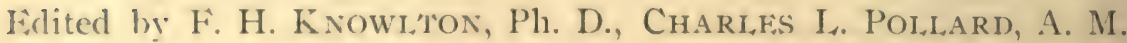

IIith the January number The IJant World enters upon its third year, nearly double in size and otherwise much improved. The articles are of wide-spread, popular interest and are written in non-technical language. Numbered among our contributors are some of the most eminent of American botanists and amateurs.

A special feature of Volume III will be an 8-page supplement issued with each number, containing a profusely illustrated, popular account of The Families of Flow = ering Plants, which will be the finest American presentation of the results of the modern study of these groups. By its aid the isolated student and amateur may become familiar with the groups of flowering plants of the world. Other features will be articles on early American botanists, plants in their relation to each other and to their surroundings, notes, news, book reviews, etc., etc.

Subscription price, including supplement, one dollar per year. Extra copies of supplement, five cents each.

Address all communications to

The Plant World Company,

P. O. Box 334, Washington, D. C.

Send for sample copy, mentioning this publication.

\section{THE ASA GRAY BULLETIN}

Edited by

THOMAS A. WILIIAMS, C. I. SHEAR, and others.

A bi-monthly Journal of Botany, devoted to the interests of Teachers, Students and Amateurs. Especial attention is given to Eidible and Poisonous Mushrooms and other CryptoGAMS. Each number contains a full page plate illustrating our North American fungi. Subscription 50 cents per year. A copy. of Dr. Burt's "KEY TO THE GENERA OF BASIDIOMYCETES," with each NEW subscription. Write for special clubbing rates.

Sample copy free. Address,

\section{THE ASA GRAY BULLETIN,}

Tacoma Park, D. C. 


\title{
THE FERN BULLETIN
}

\section{Binghamton, N. Y.}

Full of everything that will interest students and lovers of ferms. Portraits of prominent fern students, descriptions of new species, extensions of range, a monograph of the genus Equisetum illustrated by actual specimens-the latter given away to subscribers, hundreds of articles and notes on the habits, uses, folk-lore, cultivation and collection of ferns, editorials, book-news, miscellaneous notes, etc.

Every botanist should have it.

No library is complete without it

In this single journal you get the news from the whole fern world. Subscriptions 50 cents a year; sample copy free.

Send for it.

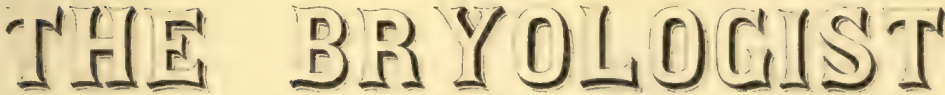

A quarterly i6 page journal devoted to the study of North American mosses. Adapted to the wants of beginners. In Igor it will contain monographs of North American genera, descriptions of new species, a series of illustrated articles on our common mosses, also a series of illustrated articles on the Lichens, and notes on the Hepatics.

50 cents a year, 15 cents a copy. Address,

Mrs. ANNIE MORRILL SMITH,

g8 Orange street, Brooklyn, N. Y.

\section{BULLETIN OF THE TORREY BOTANICAL CLUB}

\author{
Established in 1870.
}

A monthly journal devoted to scientific botany in its widest sense. Edited by I.ucien M. Lnderwood and other members of the Club. Contains annually 6ou-70u pages and about 30 plates. \$3.00 per year; single copies 20 cents.

She Torrey Botanical Club publishes at irregular intervals longer papers in the form of Memoirs, which are sold at a uniform price of $\$ 3.00$ per volume.

Address

\section{LUCIEN M. UNDERWOOD,}

COLUMBIA UNIVERSITY, NEW YORK CITY. 


\section{YOU CAN}

\section{LEARN MORE}

and in stuch a fascinating way, too, of general gardening, wild flowers and nature from MEEHANS' MON'THLY than by any' other method. It teaches in such a pleasing manner that it appeals to the taste of all intelligent persons.

MFEHANS' MON'THLY is a well illustrated, well edited horticultural magazine treating on topics of interest to every nature lover, every owner of a garden large or small, every admirer of ont-door art. It is not too technical. It is not dull.

If you have a garden and grounds, it will help make them better, prettier and more attractive. If you have none, it will help take their place and partly reconcile you to their absence. It brings nature's beanties home to you and creates a healthy interest in out-door life.

Each issue contains a magnificent colored plate of some native flower or fern, executed in the finest and most expensive style on heavy paper, Nothing as fine has ever been attempted. This is accompanied by a descriptive chapter telling in a readable way of the history and other interesting facts regarding the plant portrayed. The plate and chapter make a special feature alone well worth the subscription price. Our magazine is now completing its tenth volume.

Subscription, $\$ 2.00$ per year. Send \$1.0o for six months' trial or 20 cents for a specimen issue. No free copies. A year's subscription will make a greatly appreciated present for some nature loving friend

If you are interested in trees, shrubs and hardy plants in general, send Io cents for a pretty booklet full of helpful hints and useful suggestions. It's free with every subscription.

\section{THOMAS MEEHAN \& SONS, Publishers,}

\section{Box M,}

\section{Germantown, Philadelphia, Pa}

\section{DO YOU KNOW}

that it is as easy to study mosses as flowering plants? It is if you use MossEs WITH A HAND-LENS, the new book by Dr. A. J. Grout. No compound microscope or costly apparatus required. MONSES WITH A HAND-LENS contains descriptions of upwards of Ioo species, illustrated by 8 full page plates and 44 figures in the text, and a glossary illustrated by 46 figures.

Price \$1.10 postpaid. Send for sample pages to

Dr. A. J.GROUT',

360 Lenox Road, Brooklyn, N. Y. 




\section{LIBRARY FACIIITY OF FORESTRY}

\section{PLEASE DO NOT REMOVE \\ CARDS OR SLIPS FROM THIS POCKET}

UNIVERSITY OF TORONTO LIBRARY

S\&M

A

262 
